\title{
Innovative NIR fluorescent probes for an improved tumor detection in vivo
}

\author{
Doctoral Thesis
}

In partial fulfillment of the requirements for the degree

"Doctor rerum naturalium (Dr. rer. nat.)"

in the Molecular Medicine Study Program

at the Georg-August University Göttingen

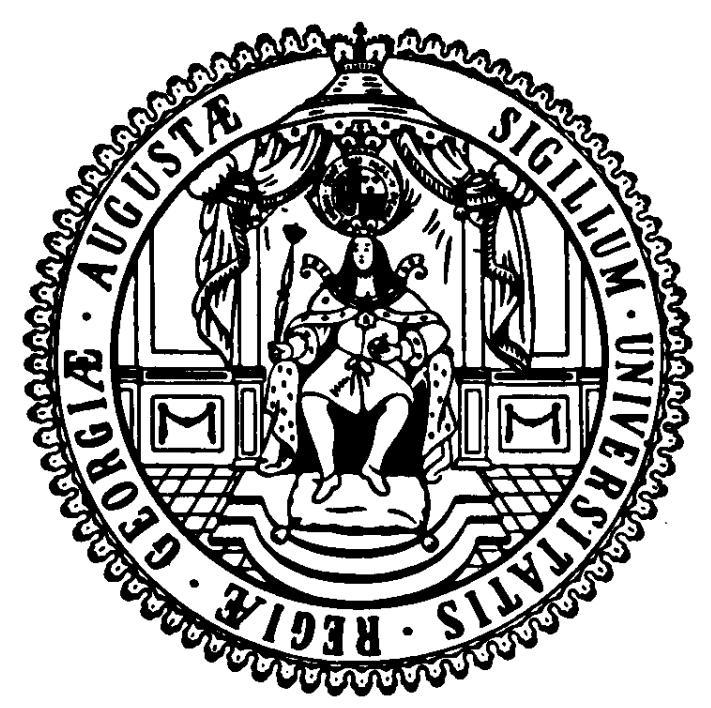

submitted by

Julia Eva Mathejczyk

born in

Braunschweig, Germany 

Für Lisa 



\section{THESIS COMMITTEE}

Prof. Dr. med. Frauke Alves (supervisor)

Email

falves@gwdg.de

Phone

0049-551-3899-655

Postal Address

Max-Planck-Institut für Experimentelle

Medizin

Dept. Molecular Biology of Neuronal Signals

Hermann-Rein Str. 3

37075 Göttingen

Prof Dr. Heidi Hahn

Email

hhahn@gwdg.de

Phone

0049-551-39-14010

Postal Address

Universitätsmedizin Göttingen

Zentrum Hygiene und Humangenetik

Institut für Humangenetik

Heinrich-Düker-Weg 12

37073 Göttingen

\section{Prof Dr. Martin Oppermann}

Email

mopperm@gwdg.de

Phone

0049-551-395822

Postal Address

Universitätsmedizin Göttingen

Zentrum Hygiene und Humangenetik

Abteilung Zelluläre und Molekulare

Immunologie

Humboldtallee 34

37073 Göttingen

Date of Disputation: December $15^{\text {th }}, 2011$ 



\section{Affidavit}

By this I declare that I independently authored the presented thesis:

\section{"Innovative NIR fluorescent probes for an improved tumor detection in vivo"}

and that I did not use other auxiliary means than indicated. Paragraphs that are taken from other publications, by wording or by sense, are marked in every case with a specification of the literary source.

Furthermore I declare that I carried out the scientific experiments following the principles of Good Scientific Practice according to the valid "Richtlinien der Georg-August-Universität Göttingen zur Sicherung guter wissenschaftlicher Praxis".

Julia Eva Mathejczyk

Göttingen, November 2011 



\section{Publications}

Pauli J, Brehm R, Grabolle M, Behnke T, Mathejczyk J, Hamann F, Alves F, Hilger I, Resch-Genger U. Dye-biomolecule conjugates and NIR-fluorescent particles for targeting of disease-related biomarkers. Proc SPIE. $2011 ; 7910,791014$

Mathejczyk JE, Pauli J, Dullin C, Napp J, Tietze LF, Kessler H, Resch-Genger U, Alves F. Spectroscopically Well-Characterized RGD Optical Probe as a Prerequisite for Lifetime-Gated Tumor Imaging. Mol Imaging. 2011 Apr 27

Napp J, Mathejczyk JE, Alves F. Optical imaging in vivo with a focus on paediatric disease: technical progress, current preclinical and clinical applications and future perspectives. Pediatr Radiol. 2011;41:161-75

Alves F, Dullin C, Napp J, Missbach-Guentner J, Jannasch K, Mathejczyk J, Pardo LA, Stühmer W, Tietze LF. Concept of a selective tumour therapy and its evaluation by near-infrared fluorescence imaging and flat-panel volume computed tomography in mice. Eur J Radiol. 2009;70:286-93

\section{PATENT APPLication}

Resch-Genger U, Behnke T, Würth C, Hoffmann K, Mathejczyk JE, Alves F, Stühmer W. Breitbandig absorbierende und emittierende NIR-Fluorophore mit großem Stokes shift für den Einsatz als Farbstoffe in core-shell-Nanopartikeln und als optische Sonden und Sensoren für die Biomarkeranalytik und als Komponenten von FRETSystemen. Patent no. 16717P-DE, submitted October 2011

\section{CONGRESS PARTICIPATION}

Molekulare Bildgebung - MoBi 2011 in Göttingen, Germany

Poster: Improvement of tumor-detection sensitivity by the use of two different data analysis methods in combination with a pH-sensitive probe. Mathejczyk JE, Dullin $\mathrm{C}$, Pauli J, Resch-Genger U, Alves F, Napp J

$2^{\text {nd }}$ poster prize

$6^{\text {th }}$ European Molecular Imaging Meeting - EMIM 2011 in Leiden, the Netherlands Poster: Lifetime imaging and lifetime quality analysis in combination with $\mathrm{pH}$ sensitive NIR fluorescence probes to improve tumor detection in vivo. Mathejczyk JE, Dullin C, Pauli J, Resch-Genger U, Alves F, Napp J 
$5^{\text {th }}$ European Molecular Imaging Meeting - EMIM 2010 in Warsaw, Poland

Poster: Characterization and evaluation of a tumor specific RGD optical probe for time-domain near-infrared fluorescence imaging. Mathejczyk JE, Resch-Genger $U$, Pauli J, Dullin C, Napp J, Tietze LF, Kessler H, Alves F 


\section{TABLE OF CONTENTS}

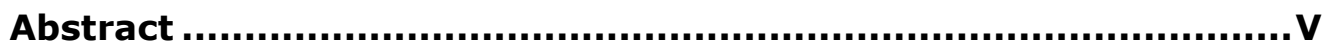

List of figures............................................................................. VI

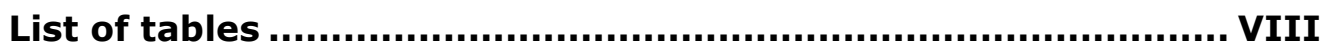

List of abbreviations ............................................................. IX

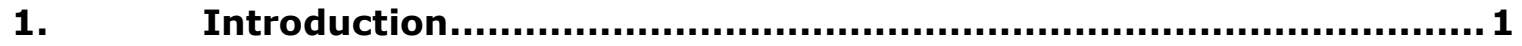

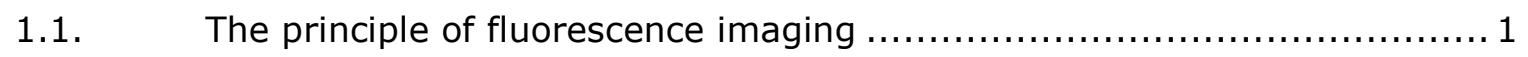

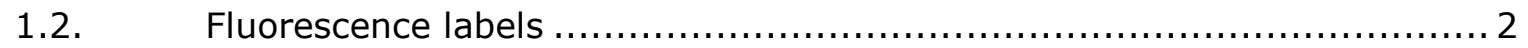

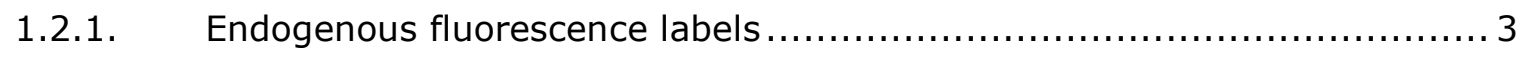

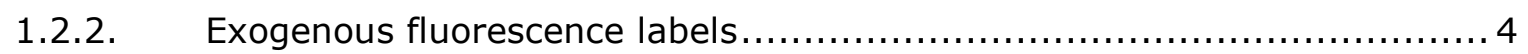

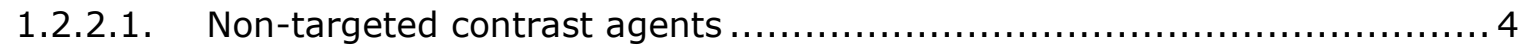

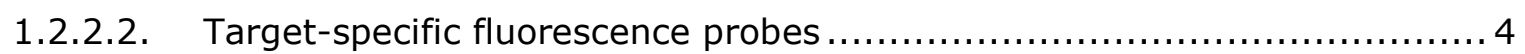

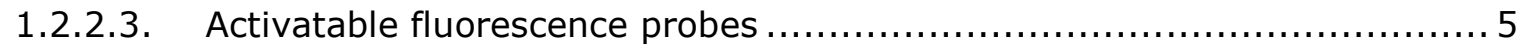

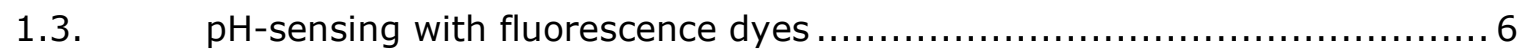

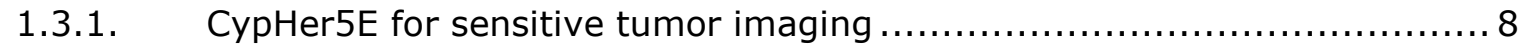

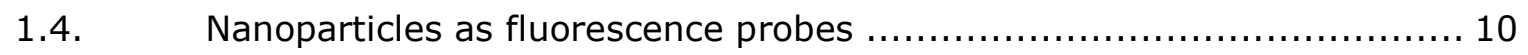

1.4.1. Polystyrene nanoparticles as potent imaging tools......................... 12

1.4.2. Highly fluorescent nanoparticles loaded with Itrybe ....................... 13

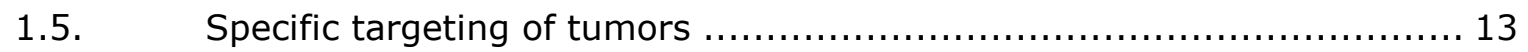

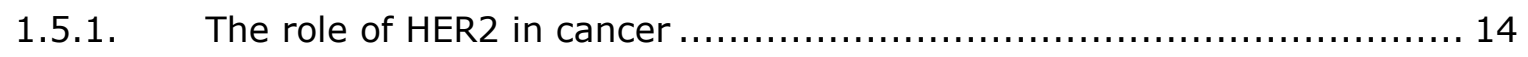

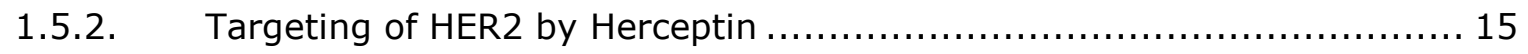

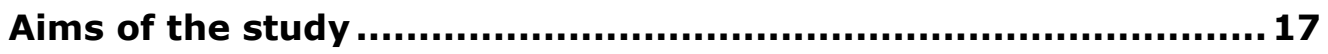

2. Material and Methods............................................................ 19

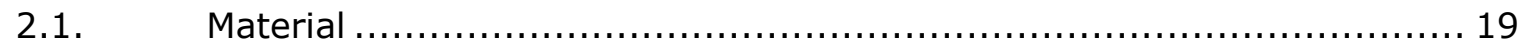

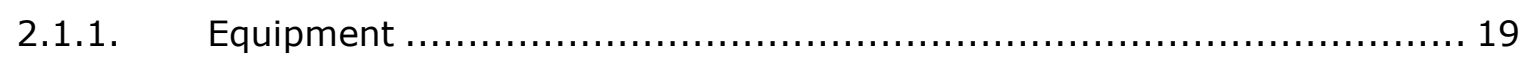

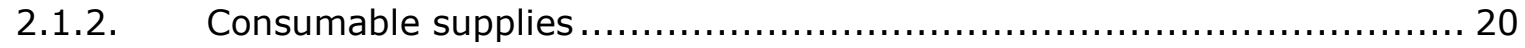

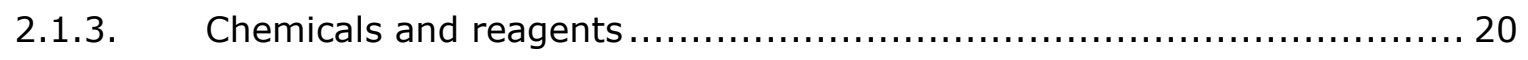

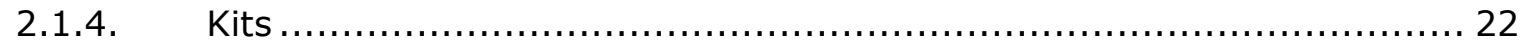

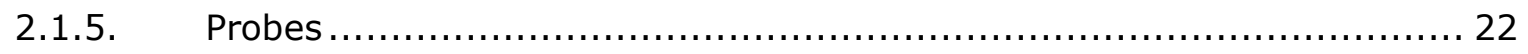

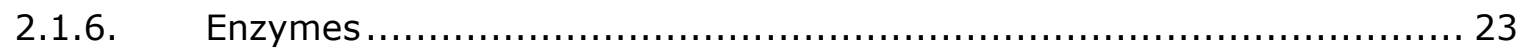




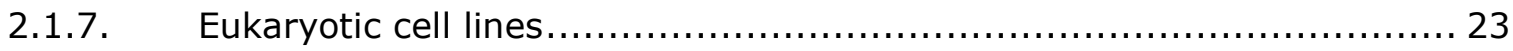

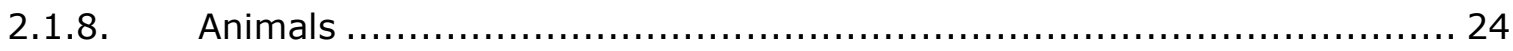

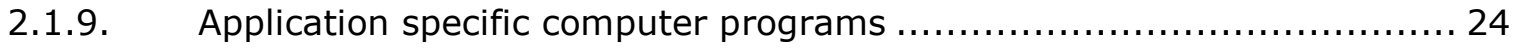

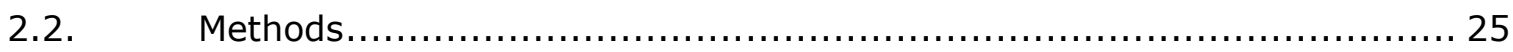

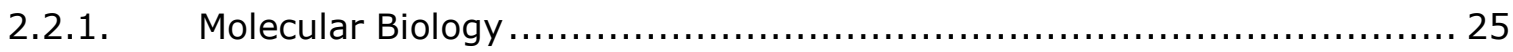

2.2.1.1. Total RNA isolation and cDNA synthesis .................................. 25

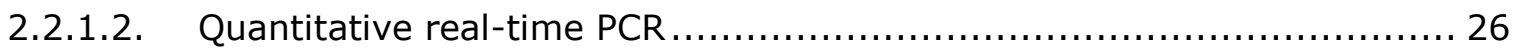

2.2.2. Protein extraction, protein separation on protein gel, and Western blot.. 27

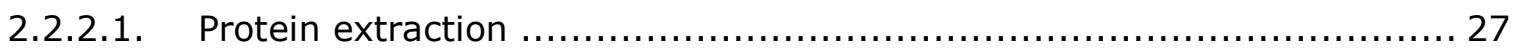

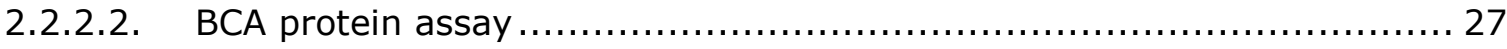

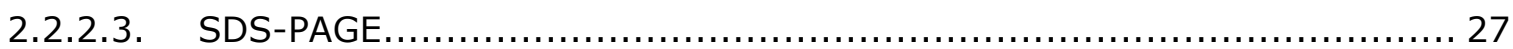

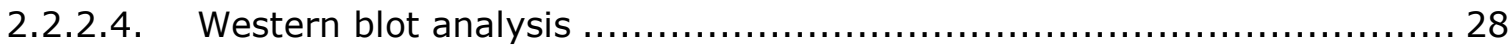

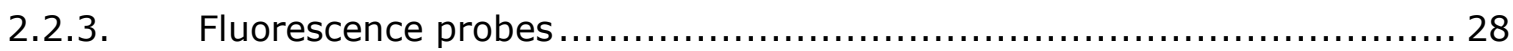

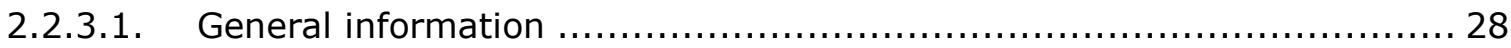

2.2.3.2. Coupling of Herceptin antibodies to fluorescence dyes .................... 29

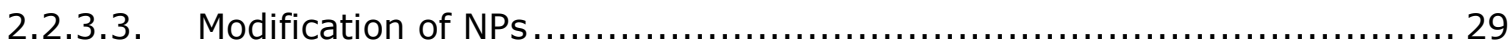

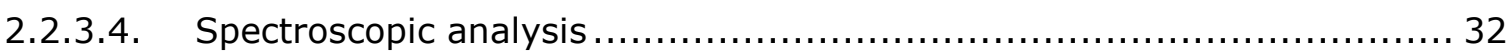

2.2.3.5. In vitro determination of fluorescence intensities ........................ 32

2.2.3.6. In vitro determination of fluorescence lifetimes........................... 33

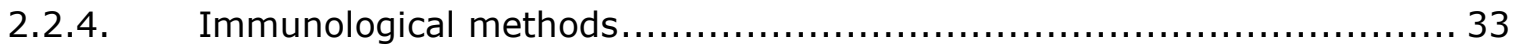

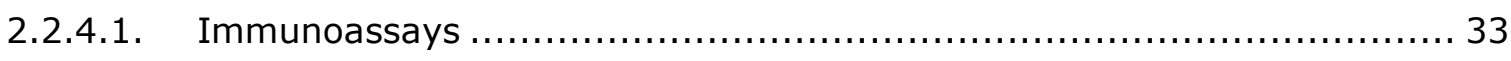

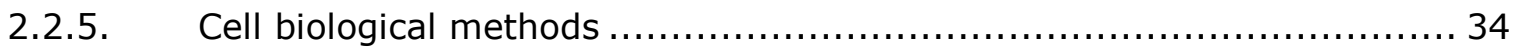

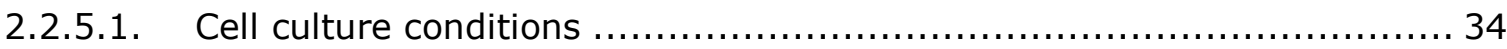

2.2.5.2. Incubation of cells with fluorescence probes ........................... 35

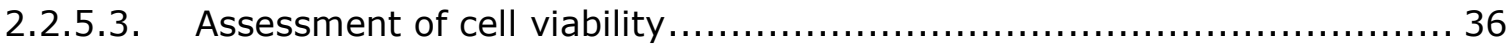

2.2.5.4. Cell preparation for animal experiments................................ 36

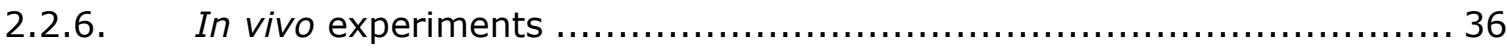

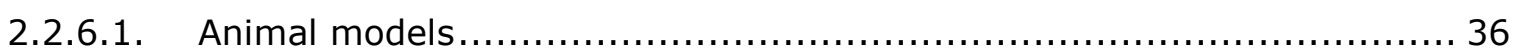

2.2.6.2. Orthotopic implantation of breast tumor cells in mice $\ldots \ldots \ldots \ldots \ldots \ldots \ldots \ldots \ldots \ldots \ldots \ldots \ldots$

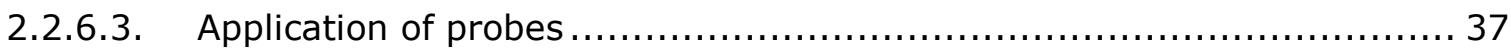




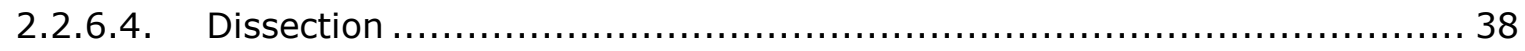

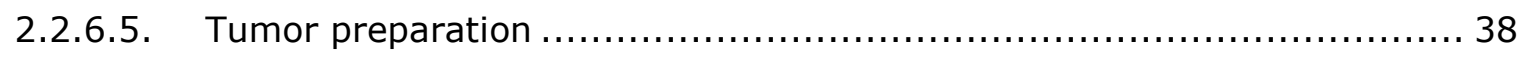

2.2.7. Histological examinations of tumor tissue................................. 39

2.2.7.1. Generation of paraffin sections from tumor tissue ......................... 39

2.2.7.2. Haematoxylin-Eosin (HE) staining of paraffin-embedded tumor sections 39

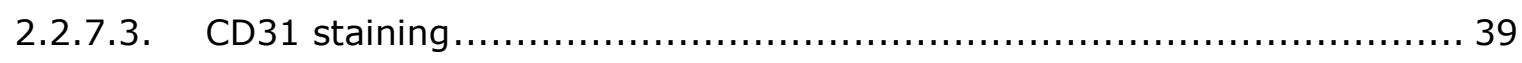

2.2.7.4. Near-infrared (NIR)-microscopic analysis of tumor sections ............... 40

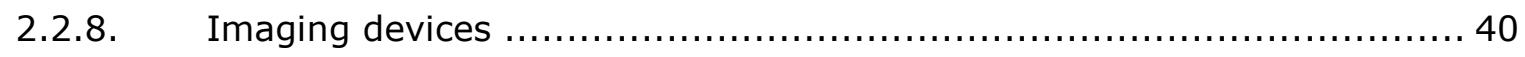

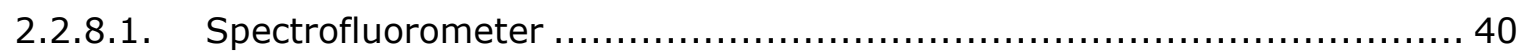

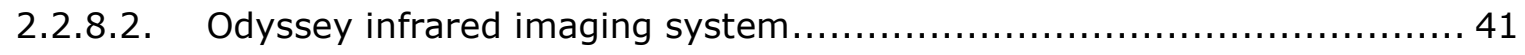

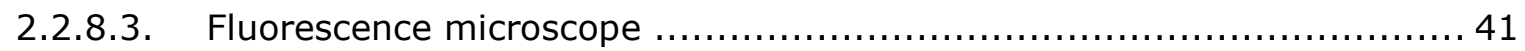

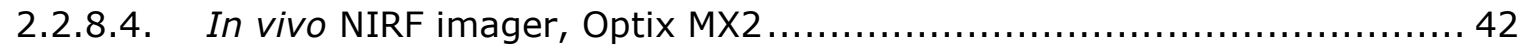

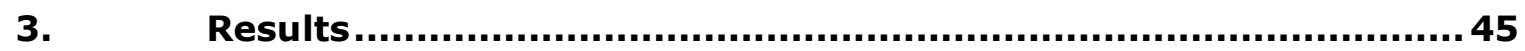

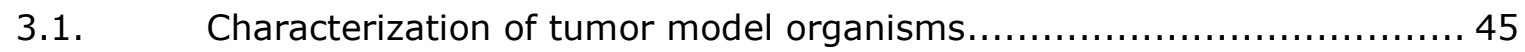

3.1.1. Orthotopic breast tumor models in nude mice ......................... 45

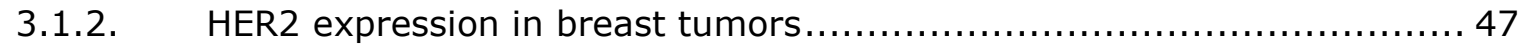

3.1.3. Validation of tumor models for evaluation of fluorescence probes......... 49

3.2. Improvement of tumor detection via $\mathrm{pH}$-sensitive NIRF probes ............ 51

3.2.1. $\mathrm{pH}-\mathrm{Her}$ conjugates at different DP ratios increase fluorescence in an acidic environment ............................................................. 51

3.2.2. LTs of fluorescence probes are influenced by the environment .............5 54

3.2.3. $\mathrm{pH}$-sensitive conjugates are functional in vitro ......................... 56

3.2.4. Improved detection sensitivity of tumors by the combined use of $\mathrm{pH}$ sensitive probes and subtraction of autofluorescence....................6 61

3.2.4.1. Background suppression via subtraction of autofluorescence in combination with $\mathrm{pH}-\mathrm{Her}$ improves tumor-detection sensitivity ...........6 61

3.2.4.2. Background suppression via LT-gating does not improve tumor-detection sensitivity in vivo .................................................. 64

3.2.5. $\mathrm{pH}-\mathrm{Her}$ conjugates at high DP ratios are less suitable for in vivo imaging68

3.3. Evaluation of Itrybe-loaded NPs as in vivo NIRF imaging agents .......... 72

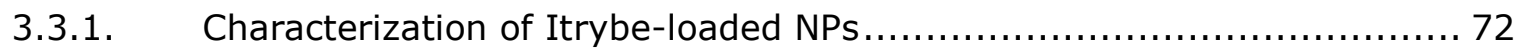




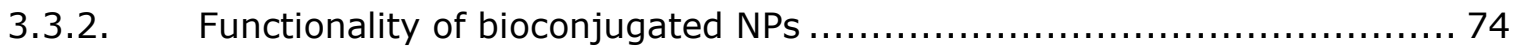

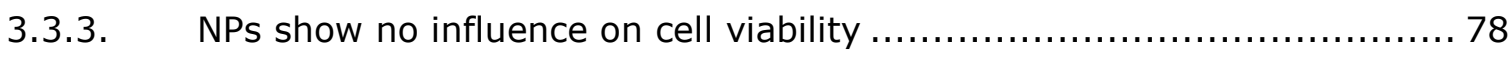

3.3.4. Binding specificity of bioconjugated NPs to breast tumor cells in vitro .... 80

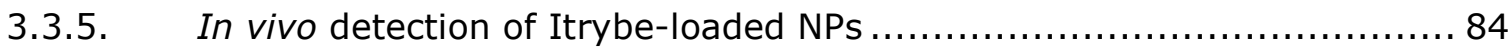

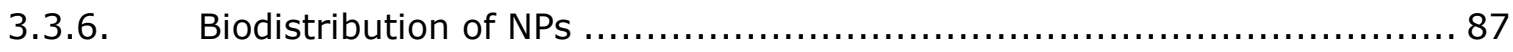

3.3.7. NPs accumulate in MDA-MB-231 breast tumors........................ 91

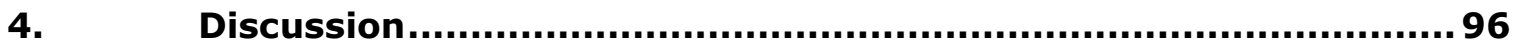

4.1. Improvement of tumor detection via $\mathrm{pH}$-sensitive NIRF probes ............96

4.1.1. Tumor imaging with target specific pH-activatable NIRF probes ........... 96

4.1.2. Suppression of background fluorescence in vivo.......................... 99

4.2. Evaluation of Itrybe-loaded PSNPs as in vivo NIRF imaging agents...... 102

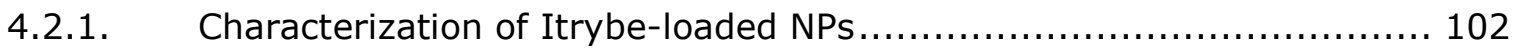

4.2.2. Detection sensitivity of Itrybe enclosed in NPs ......................... 103

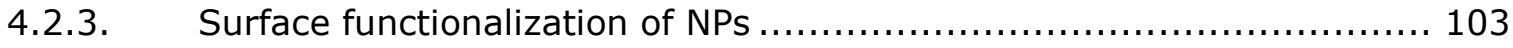

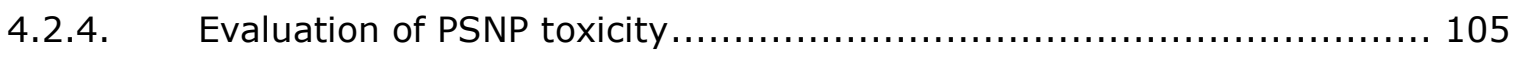

4.2.5. Binding specificity of bioconjugated NPs in vitro .......................... 105

4.2.6. Tumor targeting capacity of NPs in vivo .............................. 107

4.2.7. In vivo biodistribution of NPs ....................................... 109

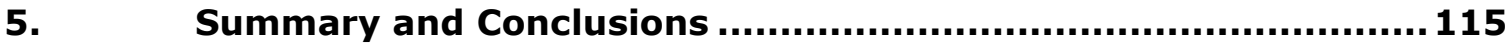

Bibliography............................................................................. 117 


\section{Abstract}

There is a great demand for the development of highly fluorescent and specific probes that enable a sensitive and early detection of malignancies and a separation from healthy tissue in a non-invasive way by in vivo imaging. The main drawback of fluorescence imaging in vivo is the autofluorescence, which hampers the detection of probe-derived signals. In this study, two novel fluorescence probes were investigated concerning their suitability for visualization of tumors in mice: a $\mathrm{pH}$-sensitive dye, CypHer5E conjugated to the tumor-specific antibody Herceptin ( $\mathrm{pH}-\mathrm{Her}$ ) as well as polystyrene nanoparticles (PSNPs) of different sizes (15, 25, and $100 \mathrm{~nm}$ ) and surface modifications $\left(\mathrm{NH}_{2}\right.$, polyethylene glycol, and Herceptin) loaded with the broadband fluorophore Itrybe. For this purpose, time-domain near-infrared (NIR) fluorescence imaging was applied in nude mice bearing orthotopic HER2-positive (KPL-4) and HER2-negative (MDA-MB-231) breast tumor xenografts. The $\mathrm{pH}-$ sensitivity and functionality of $\mathrm{pH}-\mathrm{Her}$ was confirmed by spectroscopic measurements and in cell-based assays. In HER2-positive tumor-bearing mice, $\mathrm{pH}-\mathrm{Her}$, which increases fluorescence in the acidic environment of the tumor only, combined with subtraction of autofluorescence led to an increased contrast to noise ratio (CNR) and thereby an improved sensitivity of tumor detection, when compared to the always-on fluorescent conjugate Alexa Fluor 647-Herceptin (Alexa-Her). In contrast, LT-gated imaging with $\mathrm{pH}-\mathrm{Her}$ and Alexa-Her did not improve tumor-detection sensitivity in vivo. The broad excitation and emission spectra of Itrybe enclosed in PSNPs enable excitation and sensitive detection of this dye at different wavelengths in the NIR region, in vivo. Furthermore, Herceptin-conjugated $100 \mathrm{~nm}$ NPs bind specifically to HER2 as demonstrated in immunoassays as well as on KPL-4 tumor cells and sections in vitro. However, a certain amount of unspecific binding/uptake was observed. In vivo accumulation of PEGylated NPs of all sizes was detected in MDAMB-231- but not in KPL-4 tumors as shown by ex vivo scans of excised tumors. Biodistribution analyses in healthy mice illustrated a high and rapid uptake of NPs of all sizes in the liver, accompanied by a fast elimination of NPs from the blood, thereby reducing efficient tumor accumulation. In conclusion, the $\mathrm{pH}$-sensitive dye, CypHer5E in combination with tumor-specific ligands is highly suitable for sensitive in vivo monitoring of tumors and may also be a promising tool for the detection of weak signals deriving e.g. from small metastatic lesions. Highly fluorescent Itrybe-loaded NPs have great potential for imaging applications as they provide 3D platforms for various modifications and thus help to address different biological questions in vivo. However, the bioavailability of Itrybe-loaded NPs has to be improved by modification of their surface or composition to enable efficient tumor targeting in vivo. 


\section{LIST OF FIGURES}

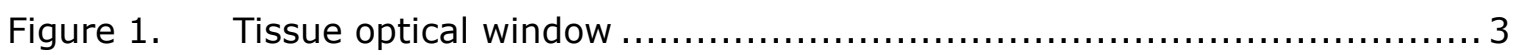

Figure 2. Improvement of tumor-detection sensitivity by the use of $\mathrm{pH}$-activatable

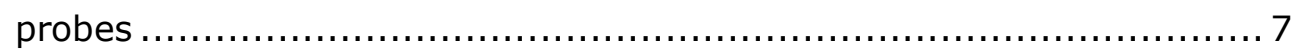

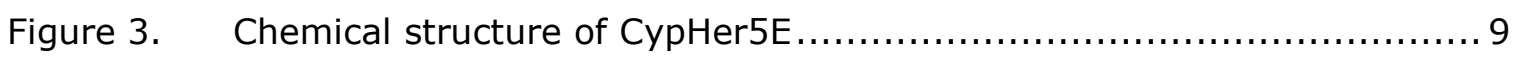

Figure 4. Absorption characteristics of CypHer5E at different $\mathrm{pH} \ldots \ldots \ldots \ldots \ldots \ldots \ldots$

Figure 5. Compositions and surface-modifications of nanoparticles............... 11

Figure 6. Transmission electron microscopy image of $100 \mathrm{~nm}$ PSNPs.............. 12

Figure 7. Normalized spectrum of wavelength of CypHer5E and Alexa Fluor 64729

Figure 8. Schematic illustration of surface-modified PSNPs....................... 30

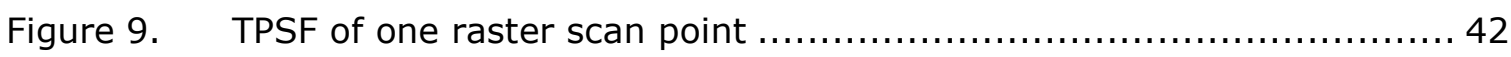

Figure 10. Macroscopic appearance of orthotopic breast tumor xenografts in nude

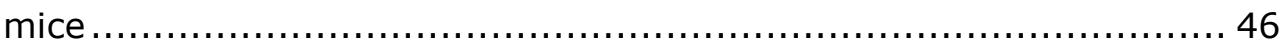

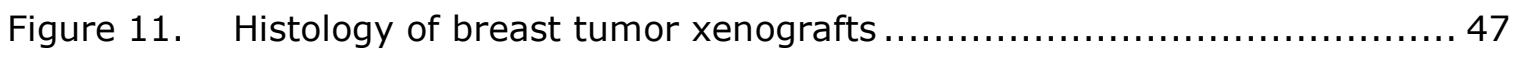

Figure 12. Expression levels of HER2 mRNA in KPL-4 and MDA-MB-231 cell lines and tumors. 48

Figure 13. HER2 protein levels in KPL-4 and MDA-MB-231 cell lines and tumors ..... 48

Figure 14. Herceptin-conjugates bind to HER2-expressing tumors in vivo .......... 50

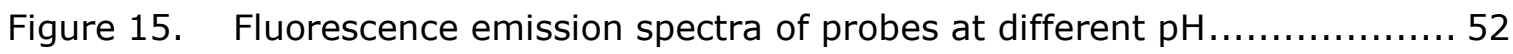

Figure 16. In vitro fluorescence microscopy demonstrates functionality of $\mathrm{pH}-$

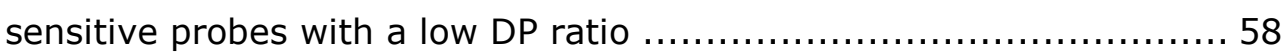

Figure 17. In vitro fluorescence microscopy demonstrates functionality of $\mathrm{pH}-$ sensitive probes with a high DP ratio 60

Figure 18. In vitro fluorescence microscopy reveals no unspecific binding of free fluorescence dyes................................................... 60

Figure 19. Intensity maps after subtraction of autofluorescence after probe application in vivo

Figure 20. Improved tumor-detection sensitivity in vivo by use of a $\mathrm{pH}$-sensitive tumor-specific probe at a low DP ratio

Figure 21. LTs of fluorescence signals in certain areas of untreated tumor-bearing mice 
Figure 22. LT-gated intensity maps after probe application in vivo .................66

Figure 23. Variations of LTs in the tumor area after probe application ..............67

Figure 24. Analysis of reliability of in vivo LT calculations ........................ 68

Figure 25. Fluorescence probes with high DP ratios reveal no improvement in

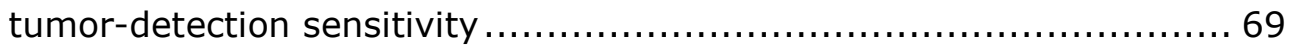

Figure 26. Detection of conjugates in a non-reducing protein gel ................. 70

Figure 27. Fluorescence excitation and emission spectra of NPs $\ldots \ldots \ldots \ldots \ldots \ldots \ldots \ldots$

Figure 28. Sensitivity of NP-encapsulated Itrybe to changes in environment ....... 74

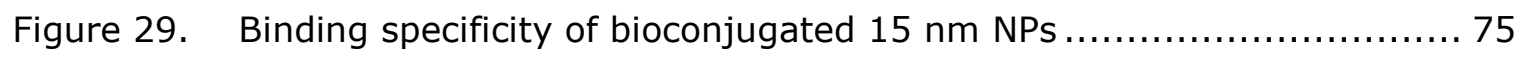

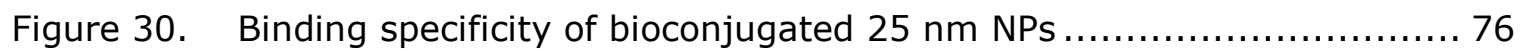

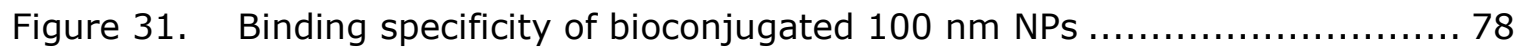

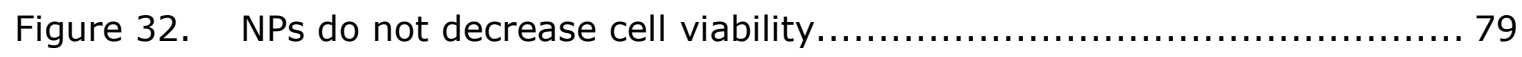

Figure 33. Increased binding/uptake of Herceptin-conjugated NPs to HER2-positive cells in vitro

Figure 34. Increased binding/uptake of Herceptin-conjugated $100 \mathrm{~nm}$ NPs in HER2positive cells over time at 37 and $4{ }^{\circ} \mathrm{C}$

Figure 35. Ex vivo fluorescence experiments demonstrate binding specificity of Herceptin-conjugated NPs to HER2-positive tumors ..................... 83

Figure 36. In vivo and ex vivo detection of NP-derived fluorescence at different wavelengths 85

Figure $37 . \quad$ In vivo detection of Itrybe-derived fluorescence $\ldots \ldots \ldots \ldots \ldots \ldots \ldots \ldots \ldots \ldots \ldots$

Figure 38. Biodistribution of NPs in healthy mice over time $\ldots \ldots \ldots \ldots \ldots \ldots \ldots \ldots . \ldots \ldots$

Figure 39. NP distribution in liver and background of healthy mice in vivo over time 90

Figure 40. Biodistribution of NPs by ex vivo analysis .............................. 91

Figure 41. Passive accumulation of PEGylated NPs in MDA-MB-231 tumors ex vivo 92

Figure 42. Detection of $15 \mathrm{~nm}$ NP-derived fluorescence in MDA-MB-231 tumor sections ex vivo 94

Figure 43. PEGylated $15 \mathrm{~nm}$ NPs can be detected in the tumor in vivo 95 


\section{LIST OF TABLES}

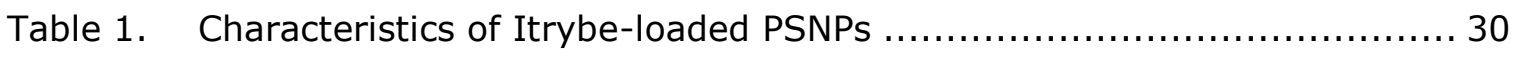

Table 2. Itrybe-loaded NPs of different sizes and surface modifications ............ 31

Table 3. Number of Herceptin molecules successfully coupled on NPs .............. 32

Table 4. Amount and application methods of fluorescent probes injected in mice. 38

Table 5. Settings used for recording of spectra of fluorescent probes ............. 41

Table 6. Microscopy filter settings used for detection of fluorophores .............. 41

Table 7. Laser and filter settings used for detection of fluorophores in the Optix

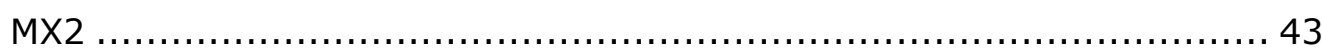

Table 8. Integration time and step sizes used for fluorescence measurements in the

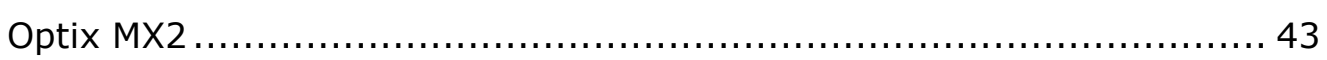

Table 9. Fluorescence intensities of Herceptin-conjugates in dependence of $\mathrm{pH} \ldots 53$

Table 10. Determination of the probes' LT under different conditions ................ 55

Table 11. Fluorescence intensities of NPs of different sizes $\ldots \ldots \ldots \ldots \ldots \ldots \ldots \ldots \ldots . \ldots \ldots$

Table 12. NP-derived signals in MDA-MB-231 tumors ex vivo....................... 93 


\section{LIST OF ABBREVIATIONS}

ADCC

AEC

Alexa-Her

Alexa-OH

ATCC

BAM

BCA

B-gal

BSA

CCD

CDNA

CD31

CNR

CT

Ct

Cy

DAPI

DEPC

DMEM

DMSO

DMSZ

DNA

dNTPs

DP

DPBS

dT

DTT

ECD

EDTA

EGFR

EPR

FAM

FCS

FDA

FMT

FRET
Antibody dependent cellular cytotoxicity

3-amino-9-ethylcarbazole

Alexa Fluor 647-Herceptin

Hydrolyzed Alexa Fluor 647

American type culture collection

Bundesanstalt für Materialforschung und -Prüfung

Bicinchoninic acid

B-d-galactosidase

Bovine serum-albumin

Charge-coupled device

Complementary DNA

Cluster of differentiation 31

Contrast to noise ratio

Computed tomoghraph(y)

Cycle threshold

Cyanine

4',6-diamidino-2-phenylindole

Diethylpyrocarbonat

Dulbecco's modified eagle medium

Dimethyl sulfoxide

Deutsche Sammlung von Mikroorganismen und

Zellkulturen

Deoxyribonucleic acid

Deoxynucleoside triphosphates

Dye to protein

Dulbecco's Phosphate Buffered Saline

Deoxythymine

Dithiothreitol

Extracellular domain

Ethylene-diamine-tetraacetic acid

Epidermal growth factor receptor

Enhanced permeability and retention

Fluorescein amidite

Fetal calf serum

U.S. Food and Drug Administration

Fluorescence-mediated (-molecular) tomography(y)

Förster (-fluorescence) resonance energy transfer 


\begin{tabular}{|c|c|}
\hline FRI & Fluorescence reflectance imaging \\
\hline GFP & Green fluorescent protein \\
\hline GI & Gastrointestinal \\
\hline HD & Hydrodynamic diameter \\
\hline HE & Haematoxylin-Eosin \\
\hline HEPES & 4-(2-hydroxyethyl)-1-piperazineethanesulfonic acid \\
\hline HER2 & Epidermal growth factor receptor 2 \\
\hline hHER2 & Human HER2 \\
\hline HRP & Horseradish peroxidase \\
\hline hTfR & Human transferrin receptor type 1 \\
\hline ICG & Indocyanine green \\
\hline IgG & Immunoglobulin $\mathrm{G}$ \\
\hline i.p. & Intraperitoneal(ly) \\
\hline i.t. & Intratumoral \\
\hline i.v. & Intravenous $(/ y)$ \\
\hline LT & Lifetime \\
\hline MPS & Mononuclear phagocytic system \\
\hline MRI & Magnetic resonance imaging \\
\hline mRNA & Messenger RNA \\
\hline NC & Normalized counts \\
\hline NIR & Near-infrared \\
\hline NIRF & Near-infrared fluorescence \\
\hline NPs & Nanoparticles \\
\hline PAGE & Polyacrylamide gel electrophoresis \\
\hline PBS & Phosphate buffered saline \\
\hline PCR & Polymerase chain reaction \\
\hline PEG & Polyethylene glycol \\
\hline PeT & Photon induced electron transfer \\
\hline PET & Positron emission tomography(y) \\
\hline $\mathrm{pH}-\mathrm{Her}$ & CypHer5E-Herceptin \\
\hline $\mathrm{pH}-\mathrm{OH}$ & Hydrolyzed CypHer5E \\
\hline PI & Total fluorescence intensity of the injected probe \\
\hline PLGA & Poly(lactic-co-glycolic acid) \\
\hline PS & Polystyrene \\
\hline PSNPS & Polystyrene nanoparticles \\
\hline QDs & Quantum dots \\
\hline RNA & Ribonucleic acid \\
\hline rpm & Rotations per minute \\
\hline
\end{tabular}


RT

RT-PCR

S.C.

SCID

SDS

SPECT

TBS

TBST

TIV

TM

TPSF

TRIS

Tween 20

US

WST1

$\lambda_{\mathrm{em}}$

$\lambda_{\mathrm{ex}}$

3D
Room temperature

Reverse transcriptase-PCR

Subcutaneous(ly)

Severe combined immunodeficiency

Sodium dodecyl sulfate

Single photon emission computed tomograph $(y)$

TRIS buffered saline

TRIS buffered saline containing Tween-20

Total fluorescence intensity in the tumor normalized to the tumor volume

Melting temperature

Temporal point spread function

Trishydroxymethylaminomethane

Polyoxyethylenesorbitan monolaurate

Ultrasonography

WST-1[2-(4-Iodophenyl)-3-(4-nitrophenyl)-5-(2,4disulfophenyl)-2H-tetrazolium]

Emission wavelength

Excitation wavelength

Three-dimensional 



\section{INTRODUCTION}

A considerable progress in the diagnostic and monitoring of cancer was the development of noninvasive imaging methods that enable detection and characterization of malignancies over time in living objects. For the anatomical illustration of tissue, structural imaging techniques are applied, like computed tomography (CT), ultra sonography (US), and magnetic resonance imaging (MRI). Characterization of cancerous tissue by its altered functionality, concerning e.g. irregular metabolic activity or altered expression of proteins is facilitated by functional imaging methods, such as positron emission tomography (PET), single photon emission computed tomography (SPECT) and optical imaging. Each technique has a combination of advantages and disadvantages affecting its selection for application in a particular study ${ }^{1}$.

Optical imaging by the use of fluorescence light has become especially attractive due to its comparatively easy use and low costs as well as high sensitivity ${ }^{2}$. In addition and in contrast to most other imaging techniques, fluorescence imaging, enables the visualization of multiple target structures or biological processes in one model organism by simultaneously using different fluorescence labels ${ }^{3}$. While the current clinical use of fluorescence imaging is mainly limited to microscopic and (micro)endoscopic techniques for diagnostic purposes and during surgical procedures, the routine application of fluorescence methods in the clinic and preclinic is certain to increase ${ }^{1}$. In recent years the use of functional tumor-specific fluorescence probes has become focus of several studies that aimed to improve sensitivity of tumor detection in tumor-bearing mice ${ }^{4-8}$. However, the majority of these probes is still limited in their specificity and sensitivity, regarding penetration depth and real-time imaging of biological processes in vivo.

Therefore, there is an ever growing demand for the development of highly fluorescent and specific as well as activatable probes that enable a sensitive and early detection of malignancies and metastatic lesions and a precise separation from healthy tissue in a non-invasive way in vivo.

\subsection{The principle of fluorescence imaging}

Fluorescence imaging relies on the detection of photons, emitted by a substance that has absorbed light of a distinct wavelength. Emission of fluorescence takes place when an orbital electron of an atom, molecule or nanostructure, after being excited to a higher quantum state, relaxes to its ground state, thereby emitting light. 
Different techniques can be applied to measure fluorescence in living organisms. Most systems are based on planar measurements of fluorescence that are usually overlaid with photographic images of the surface of the scanned sample. Normally such devices consist of a multispectral light source or a laser for excitation of the fluorescent species, appropriate filters and a device for signal detection, like a CCD camera or a photo multiplier tube. Planar imaging can be performed in an epiillumination mode, known as fluorescence reflectance imaging (FRI), in which the excitation source and the detector are located on the same side thereby measuring the returning fluorescence response. When the excitation source and the detector are placed on the opposite sides of the scanned object, imaging is performed in a transillumination mode, in which the light penetrating the object is collected ${ }^{9}$. Recent developments in tomographic fluorescence imaging including fluorescence-mediated (-molecular) tomography (FMT) provide three-dimensional (3D) information about the fluorescence distribution, as well as probe concentration and depth ${ }^{1,9-11}$.

The introduction of time-domain imaging has demonstrated a great improvement in fluorescence imaging as it allows the calculation of the fluorescence lifetime (LT) ${ }^{12}$. This parameter characterizes a fluorescence probe by describing the average time a fluorophore spends in its excited state before returning to the ground state. In this method FRI is applied with a pulsed laser diode as an excitation source. After each single excitation pulse the emitted photons are measured time-resolved using e.g. a photomultiplier tube, thereby allowing calculations of the $\mathrm{LT}^{13}$. As each fluorescence probe has a characteristic LT, the determination of LTs permits the identification of probe-derived signals, e.g. originating from different fluorescent probes ${ }^{14}$ and/or their separation from autofluorescence ${ }^{1,2,15,16}$. In vivo autofluorescence is defined as fluorescence that derives from unlabeled sources, like collagen, elastin, beta carotene, or oils, pigments, and proteins from ingested food ${ }^{17}$. This endogenous fluorescence poses one of the biggest problems in in vivo fluorescence imaging as it hampers the detection of probe-derived signals. This could be overcome by gating fluorescence intensity maps of scanned objects to the characteristic LT of the applied fluorescence probe, thereby only illustrating probe-derived signals. If the LT of the probe substantially differs from the LT of unspecific signals in this object, e.g. owing to autofluorescence, this method enables the selective illustration of only probederived signals and thus might lead to an improved sensitivity in detection of the target.

\subsection{Fluorescence labels}

For optimal detection of fluorescence light in vivo it is important to consider fluorescence probes that are very bright, relatively photostable and require minimal 
exposure to excitation light in order to sustain cell viability ${ }^{18-20}$. Of particular advantage for in vivo imaging are fluorophores absorbing and emitting in the nearinfrared (NIR) wavelength region of 650 to $900 \mathrm{~nm}$. The benefits of this spectral range are not only a decreased autofluorescence but also less absorbance by haemoglobin, water and lipids, allowing an increased tissue penetration of up to 2 $\mathrm{cm}^{1,21}$ (Figure 1). In addition, the considerably high absorption by melanin is decreasing towards longer wavelength ${ }^{1,21}$.

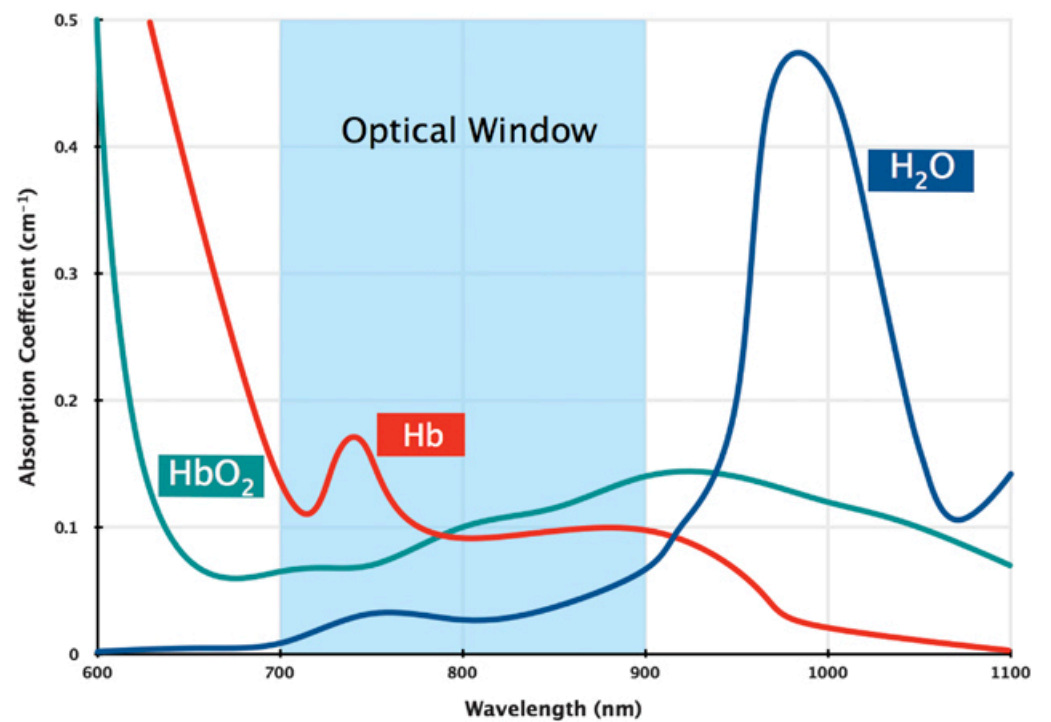

Figure 1. Tissue optical
window
The absorption (illustrated as
absorption coefficient) by major
light absorbers in tissue is
illustrated at distinct
wavelengths. In the NIR optical
window ( 700 to 900 nm; light
blue) absorption by hemoglobin
(Hb), oxygenated hemoglobin
$\left(\mathrm{HbO}_{2}\right.$ ), and water ( $\mathrm{H}_{2} \mathrm{O}$ ) is low.
$\mathrm{Picture}$ was taken from Phan et
al. ${ }^{22}$.

Different types of fluorescence labels are applied for in vivo imaging and can be divided into endogenous fluorescence labels and exogenous fluorescence probes that can further be grouped in non-targeted contrast agents, target-specific fluorescence probes, and activatable probes.

\subsubsection{Endogenous fluorescence labels}

Endogenous fluorescent labels are produced by genetic manipulation of the model organism leading to the expression of a fluorescent protein in order to visualize distinct biological functions. A break-through in the application of fluorescence imaging was the identification of the first fluorescent protein, green fluorescent protein (GFP) that was isolated from jellyfish, and purified and characterized in the early $1970{ }^{\prime 23}$. Today fluorescent proteins in many colors exist that fluoresce in the visible spectrum in colors of deep blue $(\sim 400 \mathrm{~nm})$ to far red $(\sim 670 \mathrm{~nm})$. In addition, NIR fluorescent proteins have been expressed in mammalian cells ${ }^{24,25}$. Such genetically encoded probes have been shown to be suitable for in vitro and in vivo fluorescence imaging in a variety of different cellular systems and transgenic organisms. 


\subsubsection{Exogenous fluorescence labels}

Most of the fluorescent probes are used to specifically target particular markers or molecular events in model organisms. Requirements for the efficient detection of exogenous labels are for instance, absorption- and emission-maxima in the NIR region, a high molar absorption coefficient at the excitation wavelength, a high fluorescence quantum yield, as well as sufficient thermal and photochemical stability ${ }^{19,20}$. Furthermore, suitable fluorescence dyes have to be soluble and stable in buffers and body fluids and the resulting fluorescence probe should show a good targeting efficiency with minimal nonspecific binding as well as a low toxicity ${ }^{20}$.

There are few classes of near-infrared fluorescence (NIRF) dyes available for in vivo applications. Most of them are cyanine dyes, such as indocyanine green (ICG), cypate, Alexa Fluor-dyes, or $\mathrm{Cy}^{-}, \mathrm{DY}-$, and IR-dyes ${ }^{19,26}$. Although a large variety of fluorescent probes is continuously developed and improved, ICG is the only dye approved by the FDA (Food and Drug Administration-USA) for use in patients ${ }^{27,28}$.

To characterize or monitor malignancies in vivo by the use of fluorescence probes, appropriate model organisms have to be selected. In this regard, nude mice are very suitable. These hairless mice discovered in $1962^{29}$ have a mutation in the forkhead box N1 (Foxn1) gene, leading to defective differentiation of hair and thymic disgenesis and therefore resulting in $T$ cell deficiency and partial defects in $B$ cell development ${ }^{30,31}$. As the immune system of these mice is compromised, they do not reject human tumor cells and thereby provide excellent model organisms to study tumor biology in vivo ${ }^{32}$. In addition, the lack of fur presents an advantage for in vivo fluorescence imaging as fur can cause scattering and absorption of light, hampering the detection of probe-derived signals.

\subsubsection{Non-targeted contrast agents}

Non-targeted contrast agents are designed to passively accumulate in the tissue of interest. Such probes are mainly applied for imaging of blood vessels and/or tumors. An important prerequisite for such probes is a sufficiently long blood circulation time, allowing their accumulation at the site of interest. Tumor imaging by non-targeted probes is enabled by the enhanced permeability and retention (EPR) effect, involving hyperpermeable and leaky vasculature, as well as suppression of lymphatic function ${ }^{33}$.

\subsubsection{Target-specific fluorescence probes}

Target-specific fluorescence probes are directed against molecular markers that are overexpressed or uniquely expressed on the target structure. The majority of such 
probes consists of fluorescence dyes or nanomaterials conjugates to ligands, like whole IgG antibodies, antibody fragments, or small peptides, all targeting a tissue or disease specific marker. Numerous aspects have to be considered when choosing the right ligand for the design of target-specific probes. Most important, the ligand should show maximal targeting efficiency and minimal unspecific binding ${ }^{20}$. Furthermore, the biodistribution and pharmacokinetics of ligands are strongly influenced by their size. Molecules smaller than antibodies such as antibody fragments or peptides, can be advantageous for in vivo imaging due to a rapid clearance from blood, leading to a better signal to background contrast. However, if clearance is too fast, small ligands will have insufficient time to penetrate the target tissues before being cleared from the blood. In comparison, larger whole antibodies are cleared more slowly from the blood circulation and only slowly penetrate into tissues, which may result in a high background and subsequently a poor tissue to background contrast ${ }^{34}$. Furthermore, the so called "binding site barrier", postulated by Weinstein in the early ' $90 \mathrm{~s}^{35,36}$ can influence the diffusion of ligands into the target tissue. This may be caused by a (too) high affinity and/or interaction of a particular ligand for/with its antigen, which may result in a reduced or even inhibited tumor penetration ${ }^{35}$.

\subsubsection{Activatable fluorescence probes}

Activatable fluorescence probes (also termed "smart probes") evolved to detect and monitor molecular events, such as the activity of enzymes, changes in $\mathrm{pH}$, or oxygen concentration, either by switching their fluorescence "on" or "off" or due to shifts in fluorescence absorption and/or emission spectra in response to distinct molecular processes. Such probes may be applied to selectively illustrate target structures, such as tumor tissue, based on a selective activation at the targeted tumor site only, thereby circumventing background fluorescence caused by unbound probes in the blood stream.

Probes based on the "on" and "off" principle require efficient quenching in the "off"state. The term quenching refers to processes which lead to a decrease of the fluorescence intensity of a given probe and can be achieved by distinct photochemical mechanisms, such as Förster (or fluorescence) resonance energy transfer (FRET), Hdimer formation or photon induced electron transfer (PeT).

FRET takes place between two molecules that are in close contact to each other. Here, a donor chromophore, initially in its electronic excited state, may transfer energy to an acceptor chromophore while dropping down to its ground state. FRET may occur between two fluorophores of the same type (homo-FRET) which absorb 
energy from each other (self-quenching) that would have otherwise resulted in fluorescence ${ }^{37}$, or between an excited donor fluorophore and a quencher (heteroFRET) that absorbs the energy from the donor and gives it off as heat ${ }^{6,37}$. Furthermore, some fluorophores, like ICG tend to perform autoquenching after conjugation to antibodies, most likely occurring via a noncovalent interaction between the ICG and hydrophobic amino acids on the $\mathrm{IgG}^{7,37}$. In addition, some dyes, such as Xanthene derivates at high concentrations or protein-conjugated fluorophores when in close proximity to each other, like the rhodamin dye TAMRA, tend to form $\mathrm{H}$-dimers ${ }^{5}$. This can lead to shifts of absorbance spectra resulting in quenched emission spectra ${ }^{5,37}$. In PeT, electron transfer from a PeT donor to an excited acceptor fluorophore, diminishes the fluorescence signal of the acceptor but when the donor is cleaved from the fluorophore, fluorescence occurs. Intramolecular PeT occurs when one molecule comprises an electron donor as well as an acceptor fluorophore ${ }^{37-39}$.

Activatable NIR fluorophores are applied for instance for sensing of proteases, where signals are generated as a result of lysosomal protease activity after probe internalization in cells ${ }^{1,4}$. Furthermore, activatable probes are used as substrates for bacterial $B$-d-galactosidase ( $\beta$-gal), thereby enabling the measurement of $B$-gal activity in vivo ${ }^{1,40-42}$. In addition, activatable NIR probes can be applied for sensing of metal ions, such as calcium, mercury, and zinc or for sensing of nitric oxide ${ }^{43}$ as well as oxygen ${ }^{44,45}$. NIR fluorescent lanthanide-based complexes (e.g. $\mathrm{Tb}^{3+}$ or $\mathrm{Eu}^{3+}$ ) exist that may be applied as markers of cellular stress ${ }^{43}$. Another wide application area for activatable dyes is sensing of $\mathrm{pH}$.

\section{3. $\mathrm{pH}-$-sensing with fluorescence dyes}

Although the detection of changes in $\mathrm{pH}$ is well established on tumor cells in vitro ${ }^{46}$, $\mathrm{pH}$ sensing in vivo remains challenging. A change in $\mathrm{pH}$ is a characteristic of numerous diseases like inflammation or cancer. So far only few NIR-emitting $\mathrm{pH}-$ sensitive probes exist, but most of the $\mathrm{pH}$ probes consist of fluorophores with spectra in the visible region ${ }^{1,38}$. $\mathrm{pH}$-activatable NIRF probes can be divided in two groups. The first group comprises NIRF dyes coupled to target specific proteins in either a low dye to protein (DP) ratio, resulting in autoquenching ${ }^{7}$ or in a high DP ratio resulting in self-quenching by homo $\mathrm{FRET}^{8}$. Such probes are activated by enzymatic cleavage of fluorophores, e.g. by degradation in the lysosome, allowing spatial separation from each other ${ }^{47}$. The second group consists of norcyanine- or norsquarine-based NIR pH probes which have a protonatable amine group within the fluorophore core. Examples are modified ICG, H-ICG ${ }^{43,48}$ or Square-650- $\mathrm{pH}^{49}$, and also CypHer5E, applied in this study. Accordingly, such dyes are fluorescent when protonated in an acidic 
environment but fluorescence is greatly reduced when non-protonated. Here, initiation of fluorescence is reversible, in contrast to activation via enzymatic cleavage of protein-fluorophore conjugates.

pH-responsive protonatable dyes may improve the sensitivity of detection and monitoring of acidic tumors in vivo. Following intravenous (i.v.) injection, always-on fluorescent probes systematically distribute in the entire object causing high background signals. Moreover, the tumor is undetectable until the fluorophore has concentrated in the tumor and the unbound probe is cleared from the object (Figure 2a). In contrast, $\mathrm{pH}$-activatable dyes are non-fluorescent after application, thereby causing no background fluorescence of unbound probe and only become fluorescent in the acidic tumor environment, resulting in a high tumor contrast even before the unbound (non-fluorescent) probe is cleared from the object (Figure 2b).

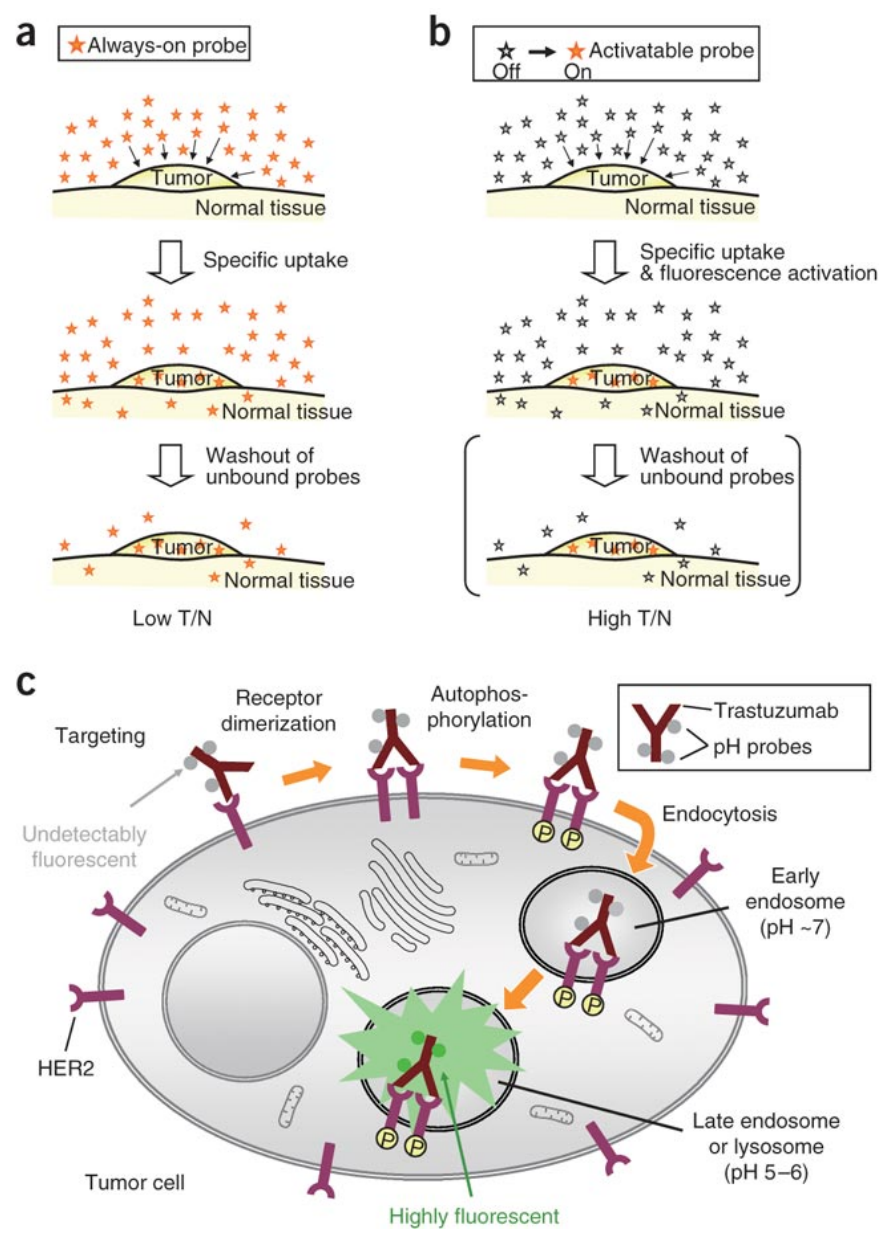

Figure 2. Improvement of tumordetection sensitivity by the use of $\mathrm{pH}$ activatable probes

a. Always-on fluorescence probes cause high background signals from the unbound probe when applied in vivo. After a certain time, following the probe's accumulation at the tumor, fluorescence intensity at the tumor site increases while background fluorescence decreases due to systemic clearance of the probe. Due to a certain amount of unbound fluorescent probe, the tumor to background ratio is low, especially at early time points after probe application. b. pH-activatable probes may avoid background fluorescence after application in vivo and only activate at the tumor site. This would result in an early detection of the fluorescent tumor even before the circulating probe is cleared from the organism, leading to a high tumor to background ratio. c. A mechanism of activation of $\mathrm{pH}$-sensitive probes at the tumor site is internalization of tumorspecific ligands via receptor-mediated endocytosis, exemplified here for Trastuzumab (Herceptin). Here, the conjugated pH-sensitive fluorophores are activated in the acidic $\mathrm{pH}$ of the late endosome and lysosome. Figure was taken from Urano et al. ${ }^{38}$. 
Activation of such $\mathrm{pH}$-sensitive probes may also occur after internalization in the targeted tumor cell. This can be achieved, for example, by conjugation of the fluorophore to a tumor-specific antibody that binds to a receptor on the targeted tumor cell, followed by internalization of the antibody-conjugate receptor complex and localization in the endosomal and lysosomal compartments. There, the low $\mathrm{pH}$ of 5 to 6 leads to activation of the dye, resulting in fluorescence (Figure 2c) ${ }^{38}$.

\subsubsection{CypHer5E for sensitive tumor imaging}

A promising candidate for in vivo imaging of tumors is the $\mathrm{pH}$-activatable NIRF dye CypHer5 (1-(5-Carboxypentyl)-2-[1E,3E)-5-(3,3-Dimethyl-5-Sulfo-1,3Dihydro-2H-Indol-2-Vlidene)-1,3-Pentadienyl]-3,3-Dimethyl-3H Indolium-5-Sulfonate $(\mathrm{V}))$. CypHer5 is a pentamethine cyanine $\left(\mathrm{Cy}^{\mathrm{TM}} 5\right)$ dye derivative that increases its fluorescence intensity with decreasing $\mathrm{pH}$. In addition, CypHer5 has a reactive functional group (hydroxyl succinimidyl ester) allowing covalent linking of proteins, like antibodies, via aliphatic amino groups. Coupling of CypHer5 to an antibody and thereby following internalization of G-protein-coupled receptor from the cell membrane into the endolysosomes was the first report of an application of a NIR pH probe ${ }^{39,43}$.

CypHer5 has been further developed to CypHer5E enhanced $\mathrm{pH}$-sensitive dye ( 4 - $\{2$ [(1E,3E,5E)-5-(3-\{6-[(2,5-dioxopyrrolidin-1-yl)oxy]-6-oxohexylis e-3methyl-5-sulfo-1,3 dihydro-2H-indol-2-ylidene)penta-1,3-dienyl]-3,3dimethyl-5-sulfo-3H-indolium-1yl\}butane-1-sulfonate) which is also commercially available. In comparison to CypHer5, CypHer5E comprises a better extinction coefficient resulting in increased excitability as well as an improved solubility by additional sulfonate groups. The structure of CypHer5E is shown in Figure 3. This $\mathrm{pH}$-sensitive dye is capable of fluorescence emission when protonated at the nitrogen atom in an acidic environment but non-fluorescent in a basic to neutral environment when the amine group in not protonated. This deprotonation leads to a shift in the absorption maximum of the dye at $645 \mathrm{~nm}$ and subsequently to a decrease in fluorescence (Figure 4). As CypHer5E has a pKa in vitro of 7.3, it is perfectly suitable for $\mathrm{pH}$ sensing in a biological environment. CypHer5E has already been shown to be functional in cell assays for studying phagocytosis ${ }^{50}$, for quantification of internalization of cellular target molecules ${ }^{51,52}$, and for monitoring of synaptic vesicle recycling ${ }^{53}$. Although CypHer5E, having NIR spectra, a good water solubility, and functional groups for bioconjugation, is a promising candidate for in vivo imaging of structures with a low $\mathrm{pH}$, like a tumor, so far no reports have been published about the use of this dye in in vivo imaging. 


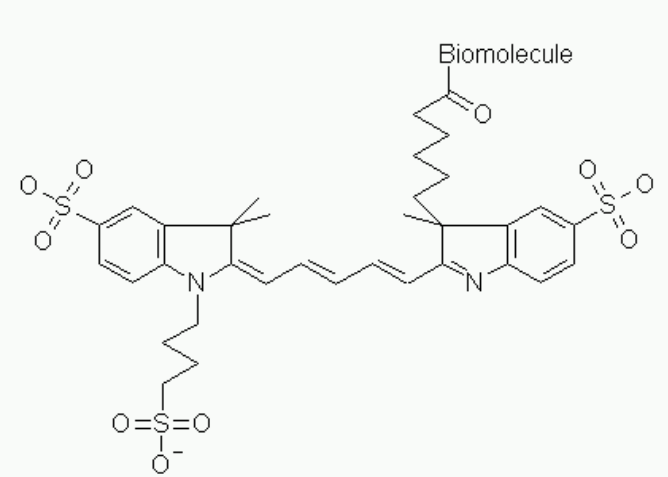

Non-Fluorescent

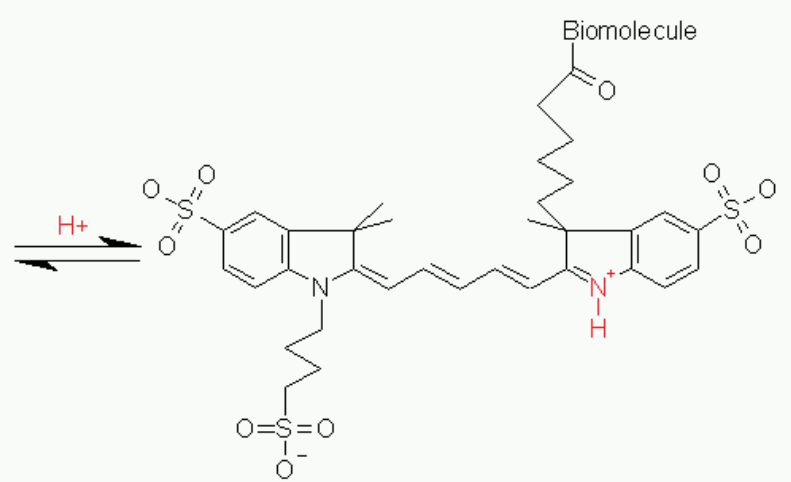

Fluorescent

\section{Figure 3. Chemical structure of CypHer5E}

In a basic to neutral solution, where the nitrogen atom of CypHer5E is not protonated, the dye is not fluorescent. When protonated in an acidic environment, the chromophore system of a classic cyanine dye is observed for CypHer5E, resulting in fluorescence. Picture was taken from GE website ${ }^{54}$.

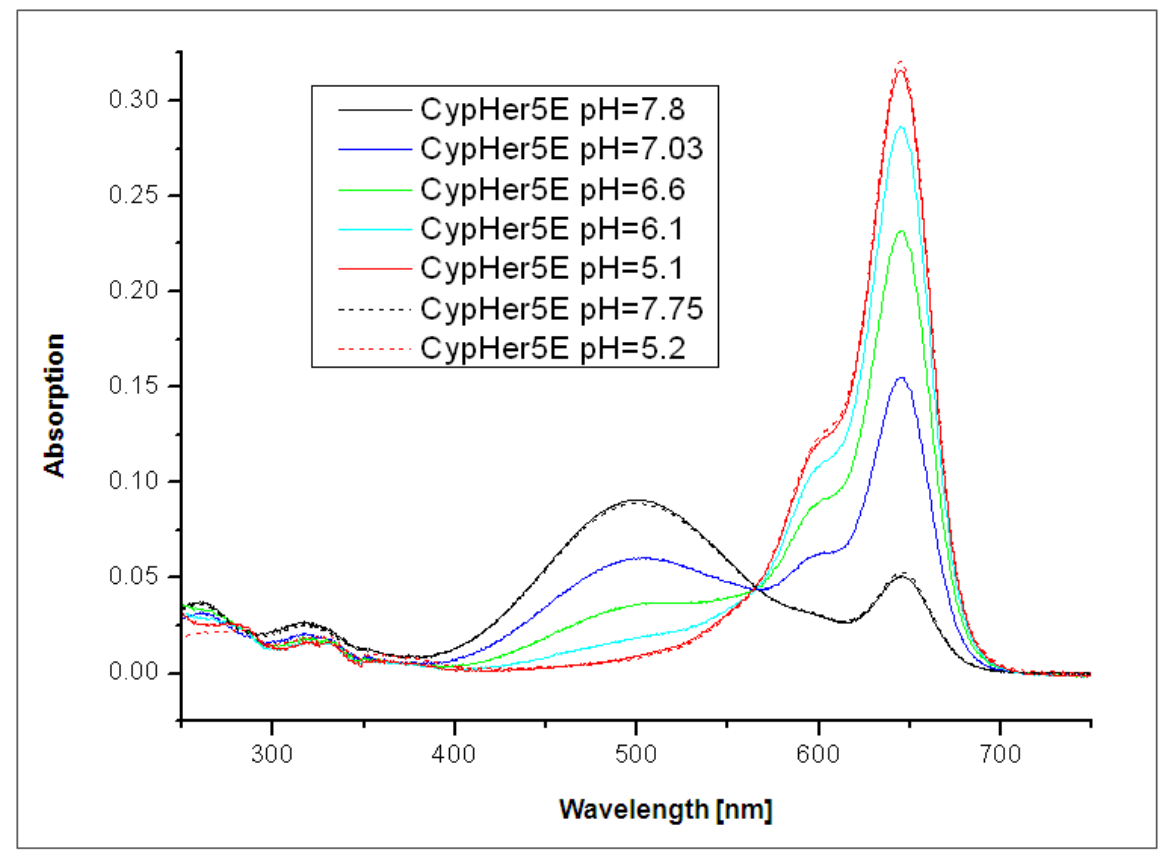

\section{Figure 4. Absorption characteristics of CypHer5E at different pH}

CypHer5E has two absorption bands that peak in the range of 490 and $645 \mathrm{~nm}$. Photon absorption around $645 \mathrm{~nm}$ results in fluorescence emission with a peak intensity at $663 \mathrm{~nm}$. The absorption band in the shorter wavelength region ( $490 \mathrm{~nm}$; not leading to fluorescence emission) increases with increasing $\mathrm{pH}$ of the PBS measurement solution, whereas the absorption band at longer wavelength ( $645 \mathrm{~nm})$ decreases resulting in decreased fluorescence. Image was provided by Jutta Pauli, BAM I.5, Berlin. 


\subsection{Nanoparticles as fluorescence probes}

Even though organic fluorophores compose the major group of fluorescence probes for in vivo applications they often suffer from limited sensitivity, a low fluorescence quantum yield, and low photostability. Moreover, organic dyes may form quenched aggregates and are sensitive to environmental changes in tissue or blood, influencing their spectroscopic properties. Furthermore, many of them possess a certain level of toxicity that hampers their application in vivo ${ }^{20}$.

A promising possibility to generate fluorescent probes for in vivo applications is to enclose fluorophores in nanomaterials, such as polymeric nanoparticles (NPs), which can be generated from a number of materials in diverse compositions and labeled with various fluorophores. This has the benefit of improved stability of the fluorophores due to reduced interactions with the environment, e.g. with quenching agents, solvent molecules, or chemically reactive species ${ }^{55}$. In reverse, by enclosement in NPs, the environment can be protected against potentially toxic fluorophores. In most cases, enclosure in NPs does not alter the dye spectral characteristics, but it minimizes dye aggregation and subsequent dye quenching, and offers an improved photostability. As NPs are capable of carrying large numbers of fluorophores, very bright fluorescence probes can be produced, compared to a single dye molecule. NPs can further be applied for simultaneous imaging by different modalities, e.g. by generating NPs that are loaded with contrast agents for MRI and surface labeled with fluorescence dyes ${ }^{56,57}$.

The core of NPs may be surrounded by a shell layer that insulates and protects the core and mediates solubility of NPs and/or linkage to bioligands ${ }^{58}$. NPs with many different configurations have been designed which, due to their large surface area can be easily modified with polymeric compounds such as polyethylene glycol (PEG) or various target-specific biomolecules, like peptides or antibodies, thereby creating highly fluorescent target-specific 3D probes for in vivo imaging ${ }^{59}$ (see Figure 5). Especially, PEGylation has a particular relevance as it can modify the pharmacokinetics of NPs by reducing their clearance through the mononuclear phagocytic system (MPS) and thereby not only increasing plasma half-lives of the NPs but also their stability and water solubility ${ }^{60-62}$.

NPs of diverse compositions have been developed for imaging purposes. Most particles can be classified into two groups: NPs that are mostly composed of organic molecules, like dendrimers ${ }^{63,64}$, liposomes ${ }^{64,65}$, poly(lactic-co-glycolic acid) (PLGA) ${ }^{66}$, polystyrene $(P S)^{67,68}$, or chitosan ${ }^{59,69}$. And those that mainly consist of inorganic materials, such as silica ${ }^{70}$, gold ${ }^{71}$, and semiconductor nanocristals, the so called 
quantum dots (QDs) that comprise a core/shell structure, such as CdSe/ZnS, $\mathrm{CdS} / \mathrm{ZnS}$, or $\mathrm{CdTe} / \mathrm{ZnS}^{72}$.

A

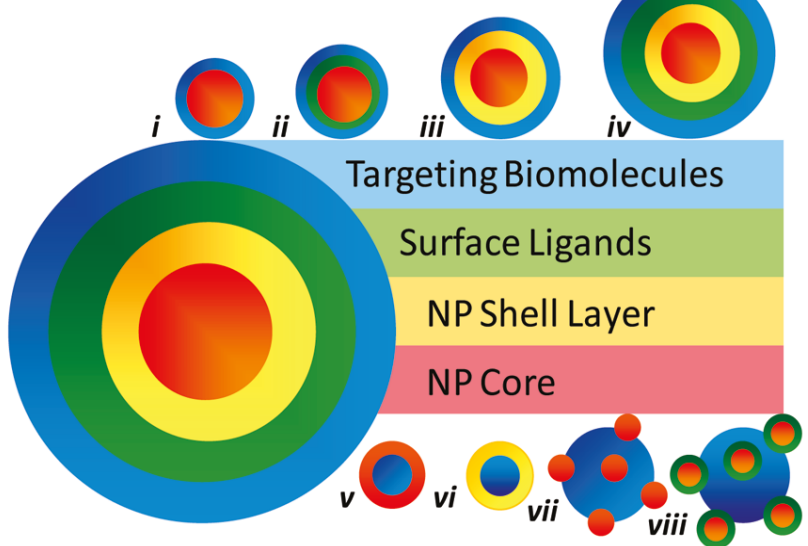

B

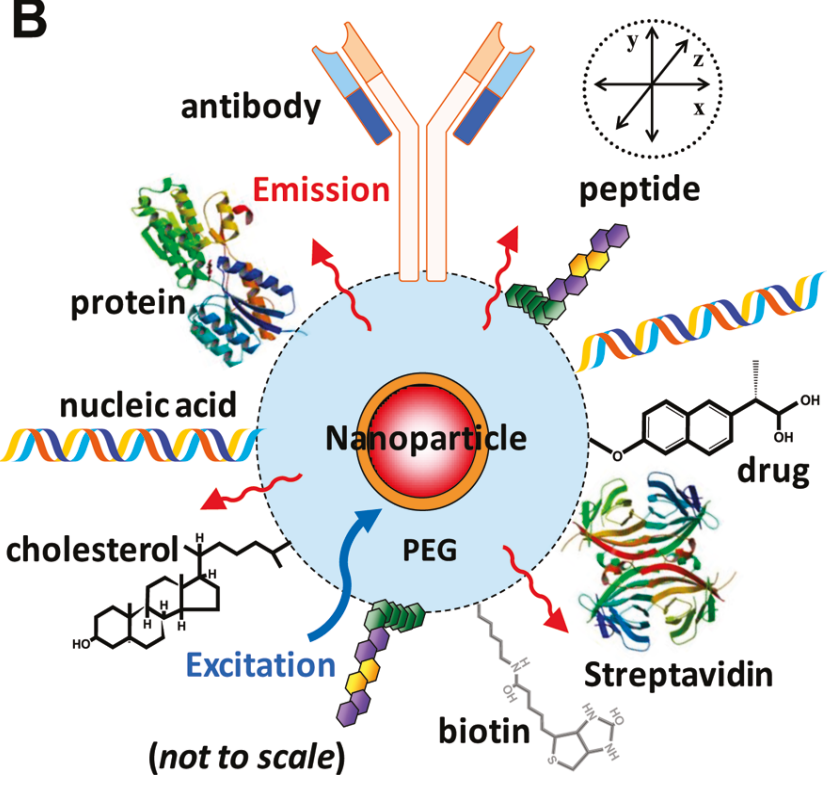

Figure 5. Compositions and surfacemodifications of nanoparticles

A. A scheme of NP components and possible configurations is shown. NPs can be composed of (i) a core directly surrounded by targeting biomolecules or (ii) surface ligands can be used to anchor the target molecules to the particle core. (iii) Biomolecules can also interact with a shell layer that surrounds the NP core or (iv) be anchored to the core/shell particle via intermediate ligands. On the other hand, (v) the targeting biomolecules can be located inside a porous NP core or ( $v i)$ be entrapped by a porous NP core surrounded by a shell layer. (vii) Furthermore, the NP core can be much smaller than the biomolecule and in addition (viii) be attached to the core by an intermediate ligand. B. A representative fluorophore-loaded NP, coated with a PEG layer is illustrated. Multiple target-specific molecules can be attached to the PEG chains in three dimensions. Specific targeting and detection of biomolecules by NPs may be achieved for instance via surface-conjugation with nucleic acids, target-specific small peptides, proteins, or antibodies. Figure was taken from Sapsford et al. ${ }^{58}$.

The composition of the NPs should be selected according to the application purpose of the particle. NPs formulated from biodegradable substances, like liposomes are especially favorable for drug delivery and are already used as chemotherapeutic vehicles in different human tumors ${ }^{64}$. Such drug vehicles are advantageous as they especially enable the delivery of hydrophobic agents, like the anti-cancer drugs paclitaxel and docetaxel that are not soluble in aqueous environments ${ }^{64}$. In addition, other biodegradable NPs, composed of chitosan, gelatin, or PLGA are being investigated as non-viral gene-delivery systems ${ }^{73}$. On the other hand, the use of non-biodegradable NPs, like PS, may be advantageous for imaging purposes as enclosed fluorophores are prevented from release into the surrounding environment, where they could potentially cause unknown toxic effects. Instead they are excreted 
from the body in the same composition as they were administered. Moreover, in the case of hydrophobic imaging agents, the incorporation in NPs enables these agents to be used in aqueous environments and consequently in vivo ${ }^{68}$.

Despite the growing effort in the development of potent NPs for diagnosis and therapy of diseases, the biodistribution, behavior and risks of NPs in living organisms have to be examined further ${ }^{74}$. In particular, most NP formulations, such as QDs ${ }^{20}$, still suffer from potentially toxic effects or from a too rapid clearance from the body due to a low transport of relatively large particles across the endothelium and/or a rapid elimination of NPs by the MPS ${ }^{75}$.

\subsubsection{Polystyrene nanoparticles as potent imaging tools}

Polystyrene nanoparticles (PSNPs; Figure 6) are especially advantageous for in vivo imaging purposes, as they are commercially available in different sizes from $15 \mathrm{~nm}$ to several micrometers. Moreover, in general PSNPs are regarded to have no impact on the metabolic activity of cells and low levels of cytotoxicity. Nevertheless, as is the case for other NP formulations, this may depend on the used concentrations of NPs and incubation times, as well as on particle size, and surface charge ${ }^{76-79}$. A further advantage is that the loading of PSNPs with inorganic and organic fluorophores is well established, thereby enabling a simple and homogeneous production of bright fluorescent reporters for imaging applications ${ }^{68}$. As PSNPs are available with various surface chemistries, this further enables a simple and efficient modification of particles with target-specific biomolecules.

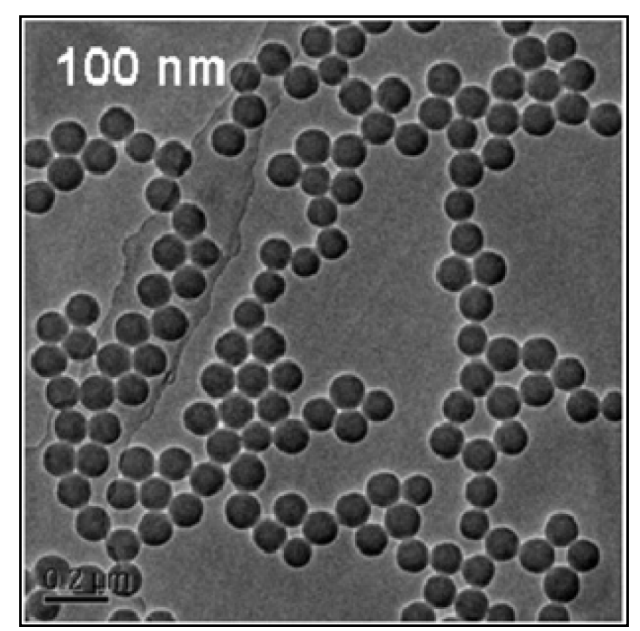

Figure 6. Transmission electron microscopy image of $100 \mathrm{~nm}$ PSNPs

The morphology of $100 \mathrm{~nm}$ PSNPs is illustrated. Scale bar represents $0.2 \mu \mathrm{m}$. Image was taken from Liu et al. ${ }^{76}$. 


\subsubsection{Highly fluorescent nanoparticles loaded with Itrybe}

A very promising dye for imaging applications in combination with NPs is the broadband fluorophore Itrybe. This hydrophobic asymmetric cyanine dye has a broad absorption (450 to $750 \mathrm{~nm}$ ) and emission spectrum (650 to $900 \mathrm{~nm}$ ) in the NIR region making it especially favorable for in vivo imaging. Furthermore, compared to other NIR fluorophores, Itrybe has a large Strokes shift which is the difference between the positions of the band maxima of the absorption and emission spectra. This enables a good separation of excitation and emission light, using appropriate filter combinations. Due to the broad spectra of Itrybe it is also possible to achieve a high compatibility of the dye with many different imaging devices equipped with various laser and/or filter settings. In contrast, most other NIR dyes are characterized by a narrow absorption- and emission spectrum and a considerably smaller Strokes shift as well as highly overlapping absorption- and emission spectra.

In contrast to other NIR fluorophores, Itrybe, does also not form quenched aggregates at high concentrations, which otherwise could result in decreased fluorescence. This allows the loading of high amounts of Itrybe molecules into solid matrices, like PS, leading to a significantly higher brightness of these probes. A further advantage of this dye as a potent imaging tool in combination with NPs, is its outstanding thermal and photochemical stability (Thomas Behnke, BAM I.5, Berlin, personal communication).

On these grounds, a patent application has been submitted by the BAM in Berlin together with the Max-Planck-Institute for Experimental Medicine and the University Medicine in Göttingen on the application of Itrybe in combination with NPs for imaging purposes (see list of publications).

\subsection{Specific targeting of tumors}

NIRF imaging offers an inexpensive and safe alternative to imaging methods like PET and SPECT which use potentially harmful, highly energetic radiation. Therefore, NIRF imaging provides an attractive tool for preclinical studies of tumor development and progression and/or evaluation of cancer therapies in animal models. As cancer is the leading cause of death worldwide ${ }^{80}$ there is a continuous need for the development of novel improved probes for an early detection and characterization of tumors as well as for a better understanding of cancer onset and progression. Cancer development is a multistep process during which cells acquire several capabilities, enabling them to become tumorigenic and finally malignant. One emerging hallmark of potential generality of cancer is reprogramming of energy metabolism which is needed to promote tumor cell growth and division ${ }^{81}$. The tumor microenvironment is known to 
be hypoxic, resulting from an inefficient perfusion due to a chaotic tumor vasculature. Under anaerobic conditions, cells favor glycolysis instead of energy production in the oxygen consuming mitochondria ${ }^{81}$. Otto Warburg first observed an abnormal behavior of energy metabolism in cancer cells, in that even in the presence of oxygen cancer cells can reprogram their energy metabolism to glycolysis ${ }^{82,83}$. The upregulation of glycolysis results in an increased acid production and subsequently in microenvironmental acidosis ${ }^{84}$. It is further known that the extracellular $\mathrm{pH}$ decreases with increasing tumor size and that gradients of interstitial $\mathrm{pH}$ exist in tumors. Moreover, the acidity can vary among different tumor types. For instance, the extracellular $\mathrm{pH}$ investigated in two breast cancer cell lines was determined to be 6.8 in MDA-MB-435 cells and 7.1 in MCF-7 cells when grown in SCID mice to a comparable size ${ }^{85}$.

The characteristic of an acidic environment, that most tumors possess, can be effectively used to increase tumor contrast by using $\mathrm{pH}$-sensitive fluorescence probes that are selectively activated in the acidic tumor environment.

\subsubsection{The role of HER2 in cancer}

Imaging probes, such as free fluorophores or dye-loaded NPs, which are systematically applied in order to detect tumors, have to be functionalized to specifically bind to tumor associated antigens on cancer cells. Easily accessible target structures might be for example membrane-associated proteins, like receptors ${ }^{86}$, ionchannels ${ }^{87}$, or cell-adhesion molecules, such as integrins ${ }^{2}$.

In the case of breast cancer detection and monitoring, a well known biomarker is the human epithelial growth factor receptor 2 (HER2, also known as c-neu, Neu or ErbB2). HER2 is highly expressed on 25 to $30 \%$ of all breast cancers ${ }^{88}$ but also in 10 to $15 \%$ of ovarian cancers ${ }^{89,90}$ and in 5 to $15 \%$ of other tumor types like gastric, cervix, colon, oral, endometrial, esophageal, lung, and pancreatic cancers ${ }^{91}$. Therefore, HER2 has become not only an interesting target structure for therapy, but due to its localization at the cell membrane, making it easily accessible by exogenously applied ligands, also an attractive marker for the diagnosis and monitoring of cancer.

HER2 is a member of the EGFR superfamily, also known as the ErbB protein family that form dimers upon ligand stimulation leading to activation of downstream signaling cascades associated with cell growth, proliferation, survival, cell motility, and angiogenesis ${ }^{91,92}$. HER2 is a $185 \mathrm{kDa}$ transmembrane glycoprotein consisting of an extracellular binding domain, a transmembrane segment, and a tyrosine kinase domain with a regulatory carboxyl terminal segment ${ }^{91,93,94}$. In addition, a truncated 
membrane-bound fragment of the HER2 protein, p95 exists that can be detected in breast tumors. p95 is generated after proteolytic cleavage of the extracellular domain of HER2 and is associated with an increased tyrosine kinase activity and thus an enhanced intracellular signaling ${ }^{95,96}$. An overexpression or amplification of HER2 results in increased intracellular cell signaling, leading to a deregulation of cell proliferation and survival, which in turn may cause uncontrolled cell proliferation and subsequently malignancy ${ }^{91}$. The prolonged duration of intracellular signaling in HER2 overexpressing cells is caused by a slowdown of the dissociation of ligand-receptor complexes which results in slower internalization of the complexes through clathrincoated pits and thereby slower degradation of the active receptors in lysosomes ${ }^{92}$.

\subsubsection{Targeting of HER2 by Herceptin}

Owing to its expression on the cell membrane of tumor cells, HER2 is an optimal target not only for therapy but also for tumor recognition. In this context a humanized monoclonal antibody, trastuzumab (Herceptin ${ }^{\circledR}$, in the following referred to as Herceptin) was developed from the murine antibody 4D5. Herceptin was approved by the FDA in 1998 for treatment of patients with metastatic breast cancer overexpressing HER2 ${ }^{91}$. In heavily pretreated patients, Herceptin has a response rate of 10 to $15 \%$ and in untreated patients of $25 \%{ }^{91}$.

Herceptin is known to bind to the extracellular domain IV of HER2 ${ }^{85}$ and to induce formation of HER2 homodimers, leading to ligand-mediated endocytosis of the receptor and to a considerable removal of HER2 from the plasma membrane and subsequently to a decreased receptor-initiated constitutive signaling ${ }^{97}$. However, the mechanism by which Herceptin causes its antitumor effect in cancer cells is not entirely understood. Other mechanisms of Herceptin action may include suppression of angiogenesis ${ }^{98}$, sterical blocking of the proteolytic cleavage of the extracellular region of HER ${ }^{99}$, and disruption of interaction of HER2 with other proteins ${ }^{99}$. Another hypothesis favors an immunological mechanism, where antibody dependent cellular cytotoxicity (ADCC) caused by Herceptin bound to the tumor cell results in tumor cell lysis ${ }^{98}$.

As Herceptin is a very well evaluated and clinically proven antibody, it presents an attractive device for the detection and monitoring of breast tumors ${ }^{100}$. Therefore, Herceptin is an optimal ligand for the bioconjugation of novel fluorescence probes, like $\mathrm{pH}$-activatable dyes and dye-loaded NPs, to be evaluated as novel probes for in vivo imaging of breast tumors.

For the evaluation of tumor-detection sensitivity of fluorescence probes tumor models are of considerable importance. Human KPL-4 breast tumor cells were 
selected as the orthotopic breast tumor model in this study as these cells are known to have an overamplification of the HER2 gene as well as a high HER2 protein expression ${ }^{101}$. Orthotopic MDA-MB-231 cell implantation was performed to obtain control tumors in nude mice that do not overexpress HER2 ${ }^{102}$. Orthotopic breast tumor xenorafts in mice generated by $\mathrm{KPL}-4^{103}$ as well as by MDA-MB-231 ${ }^{102}$ cells have already been shown to be suitable model organisms for the evaluation of Herceptin binding in vivo. Therefore, both tumor models were used in this study to investigate in vivo binding and biodistribution of Herceptin-conjuagted fluorescence probes. 


\section{AIMS OF THE STUDY}

The aim of the work presented here was to characterize and to evaluate novel and innovative fluorescent probes regarding their suitability for fluorescence imaging in vivo, in order to improve tumor-detection sensitivity in breast tumor-bearing mice.

For this purpose, 1.) a pH-sensitive NIR dye, CypHer5E and 2.) PSNPs loaded with a broadband NIR fluorophore, Itrybe, were investigated in orthotopic breast-tumor xenografts in nude mice. To achieve tumor targeting capacity, both probes were conjugated to Herceptin, in order to take advantage of the knowledge already available for the kinetics of the tumor targeting moiety Herceptin and thereby allowing me to focus on the behavior of the fluorescent labels. This is the first report of an in vivo imaging application and evaluation of CypHer5E as well as for Itrybeloaded PSNPs.

The following issues will be addressed:

1.) The suitability of $\mathrm{pH}$-activatable CypHer5E-Herceptin-conjugates to improve tumor-detection sensitivity in vivo in comparison to always-on fluorescence conjugates, AlexaFluor 647-Herceptin

a. The in vitro characterization of fluorescence probes upon the influence of different environmental factors

b. The functionality of $\mathrm{pH}$-sensitive conjugates in vitro in cell based assays

c. The capability of CypHer5E-Herceptin probes to improve tumor contrast in vivo in $\mathrm{KPL}-4$ and MDA-MB-231 breast tumor-bearing mice in combination with two different techniques for suppression of background fluorescence: subtraction of autofluorescence and LT-gated imaging

2.) The biodistribution of Itrybe-loaded PSNPs of distinct sizes (100, 25, and 15 $\mathrm{nm}$ ) and with different surface modifications (amino groups, PEG chains of different sizes, or Herceptin coupled to PEG chains) and their feasibility for application in in vivo imaging of breast tumors in mice

a. The spectroscopic characterization of Itrybe-loaded PSNPs and the influence of environmental factors on fluorescence of the probes

b. The binding of Herceptin-modified NPs to HER2 in immuno- and cellbased assays

c. The influences of NPs on cell viability 
d. The biodistribution of probes in vivo and ex vivo

e. The tumor-targeting capacity of PSNPs in KPL-4 and MDA-MB-231

breast-tumor bearing mice in vivo and ex vivo

The results of this study will contribute to an improved knowledge about the characteristics of $\mathrm{pH}$-sensitive probes and Itybe-loaded NPs and their suitability for application in non-invasive detection of breast tumors. 


\section{Material and Methods}

\subsection{Material}

\subsubsection{Equipment}

Analytical balance, Competence CP64

Anesthesia system

Apparatus for tissue processing, TP 1020

Camera, EOS 1000D

Centrifuge 5810

Chemi-Doc luminescence detection system

Centrifuge 5424

Cryostat, Jung Frigocut 2800E

Extrudate 1439

Fluorescence microscope, Axiovert 200M

Incubator, Heraeus BB6220

LightCycler 480

Light microscope, Axioscope 2

Light microscope, Telaval 31

Microtome HM 340E

Nano photometer, Pearl

Neubauer counting chamber

Odyssey infrared imaging system

Optix MX2

ORCA-ER digital camera

pH-meter, SevenEasy

Quartz glass precision cuvette

Scantainer

Spectrofluorometer, QuantaMaster

Staining cuvette

Steamer, MultiGourmet

Thermomixer compact

Tissue lyzer

Ultrasonic cleaner

Sartorius, Göttingen

VisualSonics, Toronto, Ontario,

\section{Canada}

Leica, Bensheim

Canon, Krefeld

Eppendorf, Hamburg

Bio-Rad, Munich

Eppendorf, Hamburg

Leica, Bensheim

Altromin, Lage

Carl Zeiss, Göttingen

Kendro, Laboratory products, Hanau

Roche, Mannheim

Carl Zeiss, Göttingen

Carl Zeiss, Göttingen

Thermo Scientific, Braunschweig

Implen, Munich

Schütt Labortechnik, Göttingen

Li-Cor Biosciences, Bad Homburg

ART, Montreal, Canada

Hamamatsu, Herrsching am

\section{Ammersee}

Mettler-Toledo, Giessen

Helma, Müllheim

Scanbur, Koge, Denmark

Photon Technology International,

\section{Seefeld}

VWR International, Ismaning

Braun, Kronberg

Eppendorf, Hamburg

Qiagen, Hilden

Schütt Labortechnik, Göttingen 
Vortexer, Vortex Genie 2

Wallac 1420 Victor 2 multilabel counter

\subsubsection{Consumable supplies}

Cell culture flasks

Chambered coverglasses, 4 well

Cover glasses $12 \mathrm{~mm} \varnothing$

Cryotube vials

Culture dishes

Culture plates 24-wells, 96-wells

Falcontubes $15 \mathrm{ml}, 50 \mathrm{ml}$

Filter tips, $10 \mu \mathrm{l}, 100 \mu \mathrm{l}, 1000 \mu \mathrm{l}$

Insulin syringes with integrated needle

$(30 \mathrm{G} \times 1 / 2)$

Insulin syringes with integrated needle

Insulin syringes, Omnifix 40 solo

Microscope slides

Needles, Sterican, 26G x 1/2

Hybridization transfer membranes

Nunc-Immuno modules, maxisorp loose

NuPAGE Novex 3-8 \% TRIS-acetate gels

Pasteurpipettes

Permanox cell culture slides

Serological pipettes $5 \mathrm{ml}, 10 \mathrm{ml}$

Serological pipettes $25 \mathrm{ml}$

Surgical suture materials, absorbable, Vicryl, 4/0

Tissue Tek cassettes

\subsubsection{Chemicals and reagents}

ABTS single solution
Bender \& Hobein,Zurich,

Switzerland

American Instrument Exchange, Haverhill, MA, USA

Sarstedt, Nümbrecht

Nalge Nunc International, Rochester, NY, USA

Thermo Scientific, Bonn

Nunc, Roskilde, Denmark

Sarstedt, Nümbrecht

Greiner Bio-One, Solingen

$\mathrm{BD}$, Heidelberg

Sarstedt, Nümbrecht

Braun, Melsungen

BD, Heidelberg

Braun, Melsungen

Paul Marienfeld, Lauda-Königshofen

Braun, Melsungen

Amersham/GE Healthcare, Munich

Nunc A/S, Roskilde, Denmark

Invitrogen, Darmstadt

Sarstedt, Nümbrecht

Nalge Nunc International, Naper

Ville, IL, USA

Greiner Bio-One, Solingen

Corning Incorporated, Corning, NY, USA

Johnson \& Johnson, Neuss

Vogel, Giessen

Invitrogen, Darmstadt 
AEC peroxidase substrate

Antibody diluent

Aquatex, aqueous mounting medium

Blocking buffer - fish

BSA

Casein

Chemiluminescent HRP substrate

Chloroform

Coomassie R250

DEPC

DePex, hydrophobic mounting medium

DMEM

DPBS

EDTA

Eosin G

Ethanol

FCS

Formaldehyde

GLP Nafag feed 890

Goat serum

Haematoxylin

HEPES

Hoechst 33342 trihydrochloride, trihydrate

Hydrochloric acid, 37\%

Hydrogen peroxid

Fluorescent mounting medium containing DAPI

Isoflurane

Ketamine $10 \%$

LDS sample buffer

NuPAGE Antioxidant

Paraffin (TM $60^{\circ} \mathrm{C}$ )

PBS tablets

Poly-L-lysine

Precision Plus protein standard
Vector, Peterborough, United Kingdom

Dako, Hamburg

Merck, Darmstadt

SurModics, Eden Prairie, MN, USA

Sigma-Aldrich, Seelze

Roche, Mannheim

Millipore, Billerica, MA, USA

Merck, Darmstadt

Biomol, Hamburg

Sigma-Aldrich, Seelze

Serva, Heidelberg

Gibco, Invitrogen, Darmstadt

Gibco, Invitrogen, Darmstadt

Sigma-Aldrich, Seelze

Merck, Darmstadt

Merck, Darmstadt

PAN Systems, Aidenbach

Merck, Darmstadt

Provimi Kliba AG, Kaiseraugst

Gibco, Invitrogen, Darmstadt

Merck, Darmstadt

Carl Roth, Karlsruhe

Invitrogen, Darmstadt

Merck, Darmstadt

Sigma-Aldrich, Seelze

Merck, Darmstadt

Baxter, Unterschleißheim

Medistar, Holzwickede

Invitrogen, Darmstadt

Invitrogen, Darmstadt

Süsse, Guxhagen

Gibco, Invitrogen, Darmstadt

Sigma-Aldrich, Seelze

BIO-RAD Laboratories, Munich 
ProLong gold antifade reagent with DAPI

Protease inhibitor cocktail

QIAzol lysis reagent

Reducing agent

Regime 210, U8959A01R

Sodium chloride

Sodium chloride, isotone, $0.9 \%$

Sodium hydroxide

Target retrieval solution

TRIS

TRIS-acetate SDS running buffer

Triton X-100

Tween-20

Western blot stripping buffer

WST-1 cell proliferation reagent

Xylazine 2\%

Xylol

\subsubsection{Kits}

BCA protein assay kit

Mycoplasma PlusTM PCR primer set

Ready-to-use hot-start PCR master mix

RNase-free DNase set

RNeasy mini kit

SuperScript first-strand synthesis system for RT-PCR
Invitrogen, Darmstadt

Roche, Mannheim

Qiagen, Hilden

Invitrogen, Darmstadt

Scientific Animal Food and Engineering, Augy, France

Merck, Darmstadt

Braun, Melsungen

Merck, Darmstadt

Dako, Hamburg

Sigma-Aldrich, Seelze

Invitrogen, Darmstadt

Sigma-Aldrich, Seelze

Sigma-Aldrich, Seelze

Thermo Scientific, Bonn

Clontech, Mountain View, CA, USA

Riemser, Greifswald

Merck, Darmstadt

Thermo Scientific, Bonn

Agilent Technologies, Waldbronn

Roche, Mannheim

Qiagen, Hilden

Qiagen, Hilden

Invitrogen, Darmstadt

\subsubsection{Probes}

\begin{tabular}{|c|c|c|}
\hline substance & application & provider \\
\hline Itrybe-loaded NPs & fluorescence imaging & BAM I.5, Berlin \\
\hline CypHer5E & fluorescence imaging & GE Healthcare, Munich \\
\hline Alexa Fluor 647 & fluorescence imaging & Invitrogen, Darmstadt \\
\hline $\begin{array}{c}\text { humanized anti-HER2 IgG, } \\
\text { Herceptin (Trastuzumab) }\end{array}$ & tumor targeting & $\begin{array}{c}\text { Roche Diagnostics, } \\
\text { Mannheim }\end{array}$ \\
\hline
\end{tabular}




\begin{tabular}{|c|c|c|}
\hline substance & application & provider \\
\hline $\begin{array}{l}\text { rabbit anti-HER2 IgG, } \\
\text { Neu }(\mathrm{C}-18): \mathrm{sc}-284\end{array}$ & Western blot & Santa Cruz, Heidelberg \\
\hline $\begin{array}{l}\text { goat anti-actin IgG, } \\
(\mathrm{C}-11): \text { sc-1615 }\end{array}$ & Western blot & Santa Cruz, Heidelberg \\
\hline $\begin{array}{l}\text { donkey anti-rabbit IgG, } \\
\text { HRP-labeled: NA934 }\end{array}$ & Western blot & GE Healthcare, Munich \\
\hline $\begin{array}{l}\text { rabbit anti-goat IgG, HRP- } \\
\text { labeled: } 172-1034\end{array}$ & Western blot & $\begin{array}{c}\text { BIO-RAD Laboratories, } \\
\text { Munich }\end{array}$ \\
\hline $\begin{array}{l}\text { HER2 extracellular domain } \\
\text { (ECD) protein }\end{array}$ & immunoassay & $\begin{array}{c}\text { Roche Diagnostics, } \\
\text { Mannheim }\end{array}$ \\
\hline $\begin{array}{c}\text { rat anti-mouse CD31 IgG: } \\
\text { DIA } 310 \mathrm{M}\end{array}$ & immunohistology & Dianova, Hamburg \\
\hline $\begin{array}{l}\text { goat anti-rat IgG, HRP } \\
\text { labeled: AP136P }\end{array}$ & immunohistology & $\begin{array}{l}\text { Merck, Millipore, } \\
\text { Schwalbach }\end{array}$ \\
\hline
\end{tabular}

\subsubsection{Enzymes}

Trypsine-EDTA (0.25 \%)

PAA Laboratories, Pasching, Austria

DNase I

Qiagen, Hilden

RNase $\mathrm{H}$

Invitrogen, Darmstadt

Super Script II RT

Invitrogen, Darmstadt

Uracil-DNA-glycosylase

New England BioLabs, Frankfurt

am Main

\subsubsection{Eukaryotic cell lines}

- KPL-4, provided by J. Kurebayashi, Department of Breast and Thyroid Surgery, Kawasaki Medical School, Kurashiki, Japan

Human breast cancer cell line, isolated from the malignant pleural effusion of a breast cancer patient with an inflammatory skin metastasis ${ }^{101}$.

- MDA-MB-231, American type culture collection (ATCC)/LGC, Wesel

Human breast cancer cell line, adenocarcinoma from the mammary gland derived from a metastatic pleural effusion.

- 3T3, Swiss albino mouse fibroblasts, Deutsche Sammlung von Mikroorganismen und Zellkulturen (DMSZ), Braunschweig

Fibroblasts, established from disaggregated Swiss albino mouse embryos. 


\subsubsection{Animals}

Female athymic nude mice, strain NMRI-Fox1nu/nu, Central Institution of Animal Experiments of the University Medicine, Göttingen.

\subsubsection{Application specific computer programs}

Program:

Application Software, Version 2.1

AxioVision Rel.4.6

FeliX32 Analysis 1.0

Image $1.421^{104}$

LightCycler 480 Software 1.5

OptiView 1.00.00, ART, Montreal, Canada

Quantity One 4.6.2

Wallac 1420 Manager, Version 2.0
Used for:

Odyssey infrared imaging system

Axiovert 200M fluorescence microscope

Spectroflourometer QuantaMaster Image processing

LightCycler 480

Optix MX2 in vivo imaging system Chemi-doc luminescence detection system

Victor 2 multilabel counter 


\subsection{Methods}

\subsubsection{Molecular Biology}

\subsubsection{Total RNA isolation and CDNA synthesis}

Purification of RNA from cells:

RNA from cells was isolated via use of RNeasy mini kit according to the instruction manual. Briefly, cells $\left(\sim 1 \times 10^{6}\right)$ were disrupted in buffer containing guanidine isothiocyanate and homogenized. Ethanol was then added to the lysate to bind RNA to the RNeasy silica-gel membrane and contaminants were washed away. DNase treatment was performed to eliminate genomic DNA (according to the instruction manual). After wash steps, the RNA was eluted in $30 \mu \mathrm{l}$ RNase-free diethylpyrocarbonat (DEPC) water.

\section{Purification of RNA from tumor tissue:}

RNA from tumor tissue was isolated via phenol-chloroform extraction in combination with use of RNeasy mini kit. Tumor tissue $(<100 \mathrm{mg}$ ) was homogenized with $1 \mathrm{ml}$ QIA-zol lysis reagent containing phenol and guanidine thiocyanate using a tissue lyzer. $200 \mu \mathrm{l}$ chloroform was added and samples were vortexed and incubated for 5 min at room temperature (RT). After $15 \mathrm{~min}$ of centrifugation at $12,000 \times \mathrm{g}$ at $4{ }^{\circ} \mathrm{C}$ the aqueous phase was transferred in a new cup, supplemented with $600 \mu 170 \%$ ethanol and vortexed. The suspension was transferred to a RNeasy mini spin column and centrifuged at maximal speed for $15 \mathrm{sec}$. Total RNA bound to the membrane and contaminants were washed away. DNase treatment and elution of RNA was performed as described above.

cDNA synthesis:

cDNA synthesis for reverse transcriptase-polymerase chain reaction (RT-PCR) was performed with the SuperScript first-strand synthesis system. cDNA was synthesized from $2.5 \mu \mathrm{g}$ of total RNA upon addition of $0.5 \mu \mathrm{g}$ oligo (dT) and DEPC-water to advice a RNA concentration of $0.2 \mu \mathrm{g} / \mu \mathrm{l}$ in a volume of $12 \mu \mathrm{l}$. At each step the samples were mixed by vortexing and collected by brief centrifugations. The reaction mixture was heated at $70{ }^{\circ} \mathrm{C}$ for $10 \mathrm{~min}$ and rapidly cooled on ice for $1 \mathrm{~min}$. Subsequently reaction buffer was added containing: $2 \mu$ of reverse transcriptase buffer, $2 \mu \mathrm{MgCl} 2$ $25 \mathrm{mM}, 2 \mu \mathrm{l}$ of dithiothreitol (DTT) $0.1 \mathrm{M}$ and $1 \mu \mathrm{l}$ of dNTPs $10 \mathrm{mM}$. The reaction mixture was heated for $5 \mathrm{~min}$ at $42{ }^{\circ} \mathrm{C}$. The negative samples were supplemented with $1 \mu$ l of DEPC-water, whereas to the positive samples 200 units of reverse transcriptase enzyme were added. Probes were incubated for $50 \mathrm{~min}$ at $42{ }^{\circ} \mathrm{C}$ 
following inactivation of the reverse transcriptase at $70{ }^{\circ} \mathrm{C}$ for $15 \mathrm{~min}$. To degrade RNA present in the samples 2 units of RNase $\mathrm{H}$ were incubated with the mixture for $20 \mathrm{~min}$ at $37^{\circ} \mathrm{C}$. At the end of the procedure the mixture was diluted with DEPCwater to advice a reaction volume of $25 \mu \mathrm{l}$ with a final cDNA concentration of 0.1 $\mu \mathrm{g} / \mu \mathrm{l}$.

RNA and cDNA concentration and purity were determined by optical density measurements at 260 and $280 \mathrm{~nm}$ using the spectrofluorometer.

\subsubsection{Quantitative real-time PCR}

Real-time PCR was performed using the TaqMan system in the LightCycler 480. Primers and probes were chosen from the Universal Probe Library from Roche ${ }^{105}$. The TaqMan probes contain a reporter dye fluorescein amidite (FAM) at the 5 'end of the probe and a Dark Quencher Dye at the 3'end of the probe. When cleaved by the Taq DNA polymerase during PCR the reporter and quencher dye of the probe are separated, resulting in fluorescence of the reporter. For analyzing the mRNA expression of the human epidermal growth factor receptor 2 ( $h H E R 2$ ) and human transferrin receptor type 1 (hTfR), used to control for RNA integrity and quantification, the following primers and probes were used:

Primer

$h T f R$ forward

hTfR reverse

TaqMan probe \# 61

hHER2 forward

hHER2 reverse

TaqMan probe \# 23

\section{Sequence}

5'-TTG AGA AAA CAA TGC AAA ATG TG-3'

5'-CCC AGT TGC TGT CCT GAT ATA GA-3'

5'-FAM CTG GGC AA Dark Quencher Dye-3'

5'-CAA GTA ATC CGG GGA CGA A-3'

5'-CAC TGC CCA GTT CCC TCA-3'

5'-FAM GGG CTG GG Dark Quencher Dye-3'

Real-time PCR was performed with $100 \mathrm{ng}$ CDNA, $200 \mathrm{nM}$ forward and reverse primer, $100 \mathrm{nM}$ TaqMan probe, $0.2 \mathrm{U}$ Uracil-DNA-glycosylase, and a ready-to-use hot-start PCR mix containing Taq DNA polymerase, dNTP mix, as well as buffer and $\mathrm{MgCl}_{2}$.

PCR conditions were:

$2 \min 50^{\circ} \mathrm{C} ; 10 \min 95^{\circ} \mathrm{C} ;\left(10 \sec 95^{\circ} \mathrm{C} ; 30 \sec 60^{\circ} \mathrm{C}\right.$; and $\left.10 \sec 40{ }^{\circ} \mathrm{C}\right) \times 45$ cycles. The number of PCR cycles to reach cycle threshold (Ct) was used to determine the relative mRNA expression. The results were standardized to the amount of $h T f R$ in the respective probe and to the amount of mRNA expression in the calibrator (human brain RNA). 
For this purpose the $\Delta \Delta$ Ct-Method was applied:

Normalized ratio: $2^{\Delta \mathrm{ct} 1} / 2^{\Delta \mathrm{ct} 2}$

$\triangle \mathrm{Ct} 1=\mathrm{Ct} h H E R 2_{\text {(calibrator) }}-\mathrm{Ct} h H E R 2_{\text {(sample) }}$
$\triangle \mathrm{Ct} 2=\mathrm{Ct} h T f R_{\text {(calibrator) }}-\mathrm{Ct} h T f R_{\text {(sample) }}$

\subsubsection{Protein extraction, protein separation on protein gel, and Western blot}

\subsubsection{Protein extraction}

To obtain cell lysates, cell cultures were detached from the dish as described in 2.2.5.1, transferred to a $15 \mathrm{ml}$ falcon tube, washed twice with PBS, and resuspended in $3 \mathrm{ml}$ of lysis buffer. After $30 \mathrm{~min}$ of incubation at RT, cell debris were centrifuged for $15 \mathrm{~min}$ at $18,000 \mathrm{~g}$ at $4{ }^{\circ} \mathrm{C}$ and the supernatant was used as total cell extract.

- Lysis buffer:

$50 \mathrm{mM}$ trishydroxymethylaminomethane (TRIS) $-\mathrm{HCl}, \mathrm{pH} 7.4$

$300 \mathrm{mM} \mathrm{NaCl}$

$5 \mathrm{mM}$ ethylene-diamine-tetraacetic acid (EDTA)

$1 \%$ Triton X-100

Protease inhibitor cocktail ( 1 tablet for $25 \mathrm{ml}$ )

\subsubsection{BCA protein assay}

Protein concentration was determined using the bicinchoninic acid (BCA) protein assay kit that employs bovine serum-albumin (BSA) as a standard curve. The samples (triplicates) in a volume of $25 \mu \mathrm{l}$ were incubated for $30 \mathrm{~min}$ at $37^{\circ} \mathrm{C}$ together with the BCA reagent, composed by solution A (sodium carbonate, sodium bicarbonate, bicinchoninic acid and sodium tartrate in $0.1 \mathrm{M}$ sodium hydroxide) and solution B ( $4 \%$ cupric sulfate). Absorbance was measured in the Wallac 1420 Victor 2 multilabel counter at $550 \mathrm{~nm}$.

\subsubsection{SDS-PAGE}

$50 \mu \mathrm{g}$ of total protein extract per sample were separated on a gradient SDS-PAGE (sodium dodecyl sulfate-polyacrylamide gel electrophoresis) NuPAGE Novex TRISacetate 3-8 \% gel, according to the instruction manual. Depending on the application the SDS-gels were either used for western blot analysis or stained with Coomassie for protein detection. 


\subsubsection{Western blot analysis}

For Western blot analysis the proteins were transferred to nitrocellulose hybridization transfer membranes. Membranes were blocked for $1 \mathrm{~h}$ with $0.1 \%$ casein in TRIS buffer containing Tween-20 (TBST) buffer, incubated with rabbit anti-HER2 antibody ( $1: 200$ dilution) in $0.1 \%$ casein for $1 \mathrm{~h}$, washed with deionized water for 7 times and then incubated in TBST for $5 \mathrm{~min}$. Following, the membranes were incubated with horseradish peroxidase (HRP)-coupled donkey anti-rabbit antibody (1:8000 dilution) for $1 \mathrm{~h}$. After washing, the bound antibody was visualized using chemiluminescent HRP substrate. Signals were detected in a Chemi-Doc luminescence detection system. To verify equal amounts of proteins in each loaded sample, the membranes were stained afterwards with anti-actin antibody. For this purpose, the membranes were stripped by incubation in stripping buffer at $37{ }^{\circ} \mathrm{C}$ for $20 \mathrm{~min}$ and washed and blocked as described above. The primary goat anti-actin antibody was used at a 1:200 dilution and the HRP-coupled rabbit anti-goat antibody was used at a 1:8000 dilution.

- $\underline{T B S T:}$

$140 \mathrm{mM} \mathrm{NaCl}$

20 mM TRIS- $\mathrm{HCl}, \mathrm{pH} 7.5$

$0.05 \%$ Tween-20

\subsubsection{Fluorescence probes}

In the following we analyzed properties and characteristics of activatable and alwayson fluorescence probes as well as of the fluorophore, Itrybe encapsulated in functionalized NPs, in combination with the tumor-specific antibody Herceptin.

\subsubsection{General information}

The fluorescence spectra of the $\mathrm{pH}$-sensitive hydrophilic fluorescence dye, CypHer5E can be seen in Figure 7 (left). The quantum yield of CypHer5E in PBS is 0.26 at a pH of 7.8 and 0.27 at a pH of 5.3 (experiments performed by Jutta Pauli, BAM I.5, Berlin). CypHer5E in its hydrolyzed form has a molecular weight of $751 \mathrm{~g} / \mathrm{mol}$.

The fluorescence spectra of the hydrophilic fluorescence dye Alexa Fluor 647 are depicted in Figure 7 (right). The quantum yield of the dye in PBS is 0.33 and the LT in water is $1.0 \mathrm{~ns}^{106}$. Alexa Fluor 647 in its hydrolyzed form has a molecular weight of $\sim 1200 \mathrm{~g} / \mathrm{mol}$. 

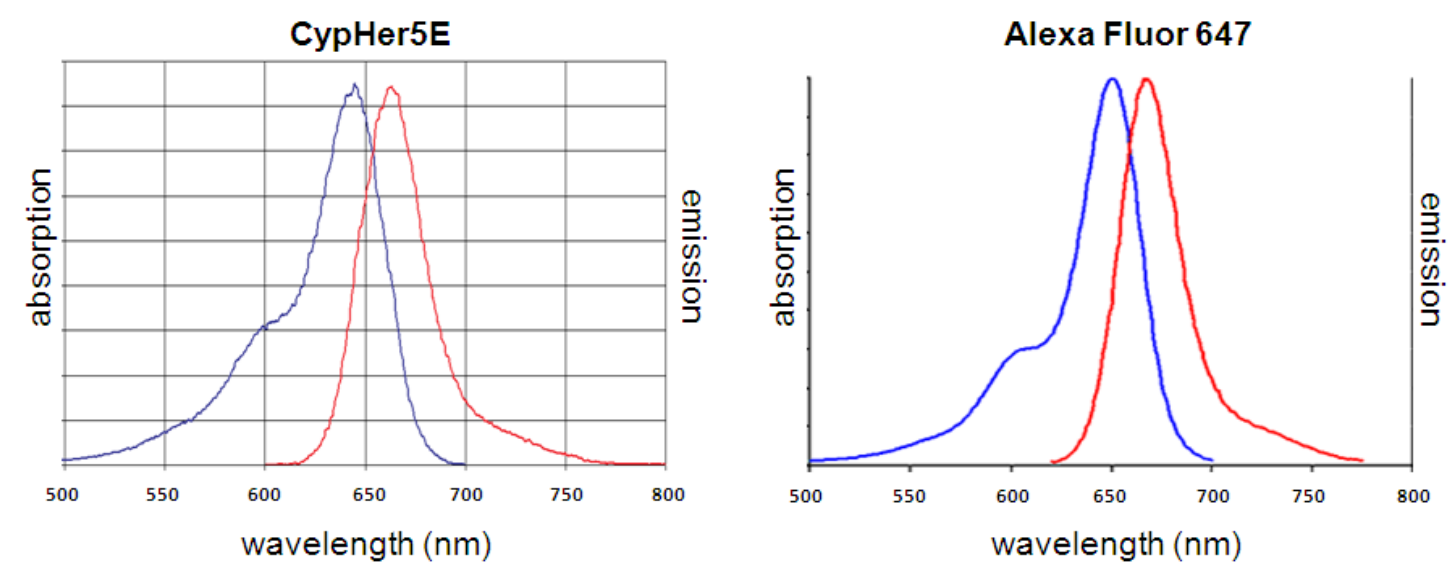

Figure 7. Normalized spectrum of wavelength of CypHer5E and Alexa Fluor 647

CypHer5E has an absorption maximum at $645 \mathrm{~nm}$ and an emission maximum at $663 \mathrm{~nm}$. Alexa Fluor 647 has similar spectra with an absorption maximum at $650 \mathrm{~nm}$ and an emission maximum at $670 \mathrm{~nm}$. Spectra were taken from GE website (left) ${ }^{54}$ and Invitrogen website (right) ${ }^{106}$ and were modified.

The hydrophobic asymmetric cyanine dye Itrybe has an absorption maximum at $\sim 590 \mathrm{~nm}$ and an emission maximum at $\sim 750 \mathrm{~nm}$. The quantum yield is 0.23 in ethanol.

\subsubsection{Coupling of Herceptin antibodies to fluorescence dyes}

Herceptin was coupled to the near-infrared fluorescence (NIRF) dyes, CypHer5E or Alexa Fluor 647, via their succinimidyl ester. Coupling resulted in conjugates with different dye to protein (DP) ratios of 0.5 to 6.4 dye molecules per antibody. Coupling was performed by Squarix Biotechnology $\mathrm{GmbH}$, Marl. Purification was done via gel chromatography followed by a preparative gel filtration. DP ratios were determined photometrically by Jutta Pauli from the Bundesanstalt für Materialforschung und -Prüfung (BAM) I.5, Berlin.

\subsubsection{Modification of NPs}

Loading of polystyrene nanoparticles (PSNPs) ${ }^{68}$ with Itrybe as well as surface modification with polyethylene glycol (PEG) and Herceptin ${ }^{107}$ were performed by Thomas Behnke, BAM I.5, Berlin. A scheme of surface-modified nanoparticles (NPs) is shown in Figure 8. For surface modification $500 \mathrm{nmol}$ PEG was added to the reaction solution per $1 \mathrm{mg}$ polystyrene (PS). For bioconjugation in a second step 0.48 nmol Herceptin was added per mg PEGylated PS. 


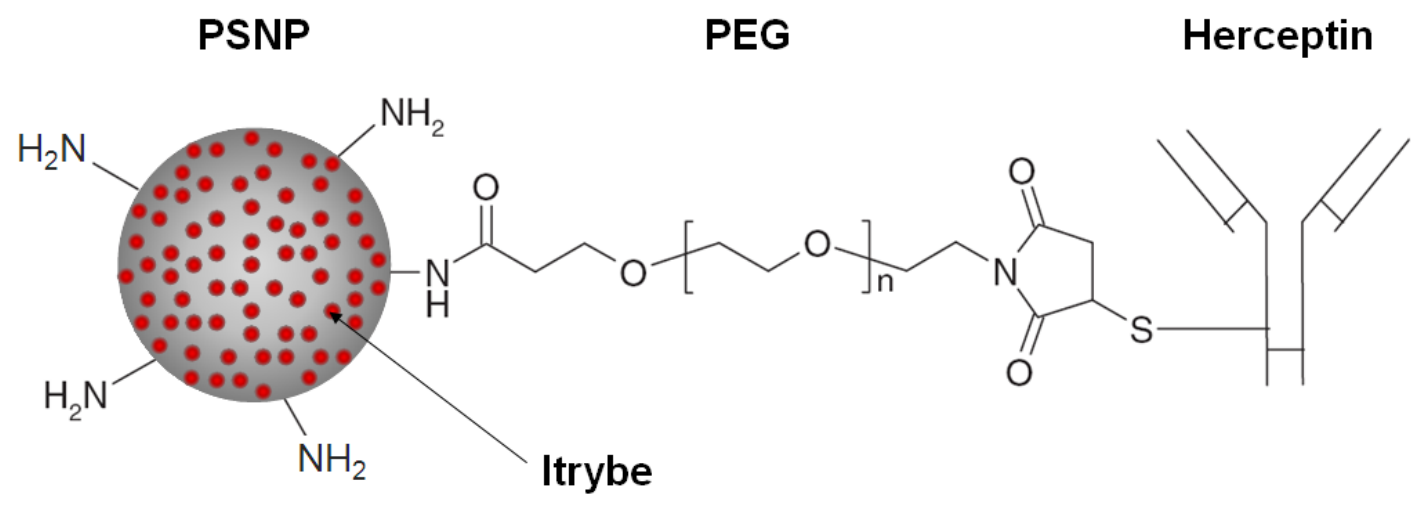

Figure 8. Schematic illustration of surface-modified PSNPs

PEG chains were coupled to Itrybe-loaded PSNPs via surface amino groups $\left(\mathrm{NH}_{2}\right)$. Thiolated Herceptin antibodies were coupled to PEG via its maleimid group in a Michael-Addition ${ }^{107}$. Figure was modified from Steinhauser et al. ${ }^{107}$.

The amounts of PSNPs used in this study are given as mg PS. Therefore, the number of particles per mg PS as well as the surface area are listed for different NP sizes in Table 1.

Table 1. Characteristics of Itrybe-loaded PSNPs

\begin{tabular}{|c|c|c|c|}
\hline size & $\mathbf{1 5} \mathbf{~ n m}$ & $\mathbf{2 5} \mathbf{~ n m}$ & $\mathbf{1 0 0} \mathbf{~ n m}$ \\
\hline $\begin{array}{c}\text { Itrybe molecules/ } \\
\text { NP }\end{array}$ & 10 & 46 & 3100 \\
\hline NPs/mg PS [1/mg] & $5.4 \times 10^{14}$ & $1.2 \times 10^{14}$ & $1.8 \times 10^{12}$ \\
\hline $\begin{array}{c}\text { relative amount: } \\
\text { NPs/mg PS* }\end{array}$ & 296 & 64 & 1 \\
\hline $\begin{array}{c}\text { surface area/ } \\
\text { mg PS }\left[m^{2} / \mathrm{mg}\right]\end{array}$ & 0.38 & 0.23 & 0.06 \\
\hline
\end{tabular}

(Calculations were performed by Thomas Behnke, BAM I.5., Berlin.)

* relative to $100 \mathrm{~nm}$ NPs

Itrybe-loaded NPs of distinct sizes and surface modifications were analyzed in this work. Table 2 shows the composition of different NPs and their abbreviations, used in this study. 
Table 2. Itrybe-loaded NPs of different sizes and surface modifications

\begin{tabular}{|c|c|c|c|c|}
\hline NP size & $\begin{array}{c}\text { surface } \\
\text { group }\end{array}$ & $\begin{array}{l}\text { PEG chain } \\
\text { size }\end{array}$ & antibody & abbreviation \\
\hline $100 \mathrm{~nm}$ & $\mathrm{NH}_{2}$ & - & - & $100 \mathrm{~nm}-\mathrm{NH}_{2}$ \\
\hline $100 \mathrm{~nm}$ & $\mathrm{NH}_{2}$ & $1.5 \mathrm{kDa}$ & - & 100 nm-PEG 1.5 kDa \\
\hline $100 \mathrm{~nm}$ & $\mathrm{NH}_{2}$ & $1.5 \mathrm{kDa}$ & Herceptin & 100 nm-PEG 1.5 kDa-Her \\
\hline $100 \mathrm{~nm}$ & $\mathrm{NH}_{2}$ & $1.5 \mathrm{kDa}$ & $\begin{array}{c}\text { 2-fold } \\
\text { amount* } \\
\text { Herceptin }\end{array}$ & 100 nm-PEG 1.5 kDa-HER x2 \\
\hline $100 \mathrm{~nm}$ & $\mathrm{NH}_{2}$ & $10 \mathrm{kDa}$ & Herceptin & 100 nm-PEG 10 kDa-Her \\
\hline $25 \mathrm{~nm}$ & $\mathrm{NH}_{2}$ & - & - & $25 \mathrm{~nm}-\mathrm{NH}_{2}$ \\
\hline $25 \mathrm{~nm}$ & $\mathrm{NH}_{2}$ & $1.5 \mathrm{kDa}$ & - & 25 nm-PEG 1.5 kDa \\
\hline $25 \mathrm{~nm}$ & $\mathrm{NH}_{2}$ & $1.5 \mathrm{kDa}$ & Herceptin & 25 nm-PEG 1.5 kDa-Her \\
\hline $25 \mathrm{~nm}$ & $\mathrm{NH}_{2}$ & $1.5 \mathrm{kDa}$ & $\begin{array}{c}\text { 5-fold } \\
\text { amount* } \\
\text { Herceptin }\end{array}$ & 25 nm-PEG 1.5 kDa-Her x5 \\
\hline $25 \mathrm{~nm}$ & $\mathrm{NH}_{2}$ & $10 \mathrm{kDa}$ & - & $25 \mathrm{~nm}-\mathrm{PEG} 10 \mathrm{kDa}$ \\
\hline $25 \mathrm{~nm}$ & $\mathrm{NH}_{2}$ & $10 \mathrm{kDa}$ & Herceptin & 25 nm-PEG 10 kDa-Her \\
\hline $15 \mathrm{~nm}$ & $\mathrm{NH}_{2}$ & - & - & $15 \mathrm{~nm}-\mathrm{NH}_{2}$ \\
\hline $15 \mathrm{~nm}$ & $\mathrm{NH}_{2}$ & $10 \mathrm{kDa}$ & - & $15 \mathrm{~nm}-\mathrm{PEG} 10 \mathrm{kDa}$ \\
\hline $15 \mathrm{~nm}$ & $\mathrm{NH}_{2}$ & $10 \mathrm{kDa}$ & $\begin{array}{c}\text { 5-fold } \\
\text { amount* } \\
\text { Herceptin }\end{array}$ & 15 nm-PEG 10 kDa-Her x5 \\
\hline $15 \mathrm{~nm}$ & $\mathrm{NH}_{2}$ & $1.5 \mathrm{kDa}$ & $\begin{array}{c}\text { 5-fold } \\
\text { amount* } \\
\text { Herceptin }\end{array}$ & 15 nm-PEG 1.5 kDa-Her x5 \\
\hline $15 \mathrm{~nm}$ & $\mathrm{NH}_{2}$ & $\begin{array}{c}1 / 10 \\
\text { amount* } \\
1.5 \mathrm{kDa}\end{array}$ & $\begin{array}{c}\text { 5-fold } \\
\text { amount* } \\
\text { Herceptin }\end{array}$ & 15 nm-PEG 1.5 kDa-1/10-Her x5 \\
\hline
\end{tabular}

$* X / X$ amount and $X$-fold amount refers to variations in the amount of PEG (500 nmol/mg PS) or Herceptin $(0.48 \mathrm{nmol} / \mathrm{mg} \mathrm{PS})$ used for coupling procedure.

The amount of Herecptin that was successfully coupled to the surface of $100 \mathrm{~nm}$ particles was determined in a BCA assay following measurement of absorption spectra and correction for absorbance by Itrybe and scattering of the particles. Experiments were performed by Thomas Behnke, BAM I.5, Berlin. Table 3 shows the amount of Herceptin molecules per 100 nm NP used in this study. 
Table 3. Number of Herceptin molecules successfully coupled on NPs

\begin{tabular}{|c|c|c|}
\hline probe & $\begin{array}{c}\text { Herceptin molecules/ } \\
\text { NP }\end{array}$ & $\begin{array}{c}\text { Herceptin molecules/ } \\
\text { mg PS }\end{array}$ \\
\hline $100 \mathrm{~nm}$-PEG 1.5 kDa-Her & $\sim 70$ & $1.3 \times 10^{14}$ \\
\hline $100 \mathrm{~nm}$-PEG $1.5 \mathrm{kDa}$-Her $\mathrm{x} 2$ & $\sim 100$ & $1.8 \times 10^{14}$ \\
\hline $\begin{array}{c}100 \mathrm{~nm}-\text { PEG } 10 \mathrm{kDa}-\mathrm{Her}, \\
\text { probe } 1 *\end{array}$ & $\sim 10$ & $1.8 \times 10^{13}$ \\
\hline $\begin{array}{c}100 \mathrm{~nm}-\text { PEG } 10 \mathrm{kDa}-\mathrm{Her}, \\
\text { probe } 2 *\end{array}$ & $\sim 10$ & $1.8 \times 10^{13}$ \\
\hline
\end{tabular}

* probe 1 and 2 refers to two independently produced probes.

\subsubsection{Spectroscopic analysis}

Fluorescence conjugates:

As dyes were coupled to Herceptin at different DP ratios, the amount of $\mathrm{pH}-\mathrm{Her}$ and Alexa-Her used in the study always refers to the amount of Immunoglobulin G (IgG) in the respective conjugates. Fluorescence emission spectra of CypHer5E-Herceptin (in the following referred to as $\mathrm{pH}-\mathrm{Her}$ ) and Alexa Fluor 647-Herceptin (in the following referred to as Alexa-Her) conjugates were measured in a concentration of 5 $\mu \mathrm{g}$ IgG in $75 \mu \mathrm{l}$ phosphate buffered saline (PBS; $0.07 \mathrm{~g} / \mathrm{l}$ ). In order to evaluate the influence of $\mathrm{pH}$-changes on fluorescence intensity of the conjugates they were measured in a solution with a $\mathrm{pH}$ of 7.5 and 5.5. The $\mathrm{pH}$ of PBS was adjusted with 1 $\mathrm{M} \mathrm{NaOH}$ and $1 \mathrm{M} \mathrm{HCl}$ solution and $\mathrm{pH}$ values were controlled again after addition of fluorescence probes and adjusted again to the respective value if necessary.

Itrybe-loaded NPs:

Excitation and emission spectra of Itrybe-loaded NPs with different surfacemodifications and sizes were recorded. For this purpose $0.5 \mathrm{~g} / \mathrm{l}$ of $100 \mathrm{~nm}$ and $25 \mathrm{~nm}$ NPs and $2 \mathrm{~g} / \mathrm{l}$ of $15 \mathrm{~nm}$ NPs were measured in $75 \mu \mathrm{H}_{2} \mathrm{O}$.

Measurements were performed in a spectrofluorometer. The settings used for recording of spectra are listed in section 2.2.8.1.

\subsubsection{In vitro determination of fluorescence intensities}

Fluorescence intensities of Itrybe-loaded NPs were measured to analyze influences of the environment on fluorescence of NPs. Itrybe-loaded NPs were measured in PBS or PBS containing $5 \%$ BSA with a pH of 7.5 and 5.5, respectively. The $\mathrm{pH}$ value of the solutions was adjusted as described in 2.2 .3 .4 . For measurement $0.25 \mathrm{~g} / \mathrm{l}$ of $100 \mathrm{~nm}$ and $25 \mathrm{~nm}$ and $0.5 \mathrm{~g} / \mathrm{l}$ of $15 \mathrm{~nm}$ NPs in $150 \mu \mathrm{l}$ of the respective solution were placed 
in 96-well plates. Measurements were done in the Optix MX2 as described in section 2.2.8.4.

\subsubsection{In vitro determination of fluorescence lifetimes}

Fluorescence lifetime (LT) analyses were performed in vitro in order to identify probe-derived signals in vivo according to their LT. LTs of the fluorescence conjugates $\mathrm{pH}-\mathrm{Her}$ and Alexa-Her as well as of the respective free, hydrolyzed fluorescence dyes (in the following referred to as $\mathrm{pH}-\mathrm{OH}$ and Alexa-OH) were determined in PBS or PBS containing $5 \%$ BSA with a pH of 7.5 and 5.5, respectively. The $\mathrm{pH}$ of the solutions was adjusted as described in section 2.2.3.4. For measurement $3 \mu \mathrm{g}(0.02 \mathrm{~g} / \mathrm{l}) \mathrm{IgG}$ or $0.03 \mathrm{nmol}(0.2 \mathrm{mM})$ free dye in $150 \mu \mathrm{l}$ of the respective solution were placed in 96-well plates. Measurements were performed in the Optix MX2 as described in section 2.2.8.4.

\subsubsection{Immunological methods}

\subsubsection{Immunoassays}

Functionality of Herceptin after surface-conjugation to Itrybe-loaded NPs was evaluated via immunoassays. Here, specific binding to antigen-coated plates was analyzed and compared to the unspecific binding to uncoated plates and to binding of non-Herceptin-modified NPs. The extracellular domain of HER2, in the following referred to as HER2 antigen, was used as antigen.

All steps of the immunoassay were performed at RT. 96-well plates were either coated over night with $2.5 \mathrm{~g} / \mathrm{l}$ HER2 antigen in $70 \mu \mathrm{l}$ TRIS buffered saline (TBS) buffer or treated only with TBS. Unspecific binding sites were blocked with $3 \%$ BSA in TBS for $1 \mathrm{~h}$. NP-probes were added to the wells at different concentrations (from 0.02 to $2 \mathrm{~g} / \mathrm{l}$ ) in $70 \mu \mathrm{l} \mathrm{TBS}$. For competitive binding experiments a mixture of $14 \mu \mathrm{g}$ Herceptin-labeled NP probe $(0.2 \mathrm{~g} / \mathrm{l})$ and increasing amounts of free Herceptin $(0.005$ to $1 \mu \mathrm{g}$ ) in $70 \mu \mathrm{l}$ TBS were added per well. Probes were incubated for $2 \mathrm{~h}$. After three washing steps with TBST (see section 2.2.2.4) the wells were covered with $100 \mu \mathrm{l}$ TBS and fluorescence was directly measured in the Odyssey infrared imaging system.

- TBS:

$140 \mathrm{mM} \mathrm{NaCl}$

20 mM TRIS- $\mathrm{HCl}, \mathrm{pH} 7.5$ 


\subsubsection{Cell biological methods}

\subsubsection{Cell culture conditions}

Cultivation:

KPL-4, MDA-MB-231, and 3T3 cells were propagated in standard Dulbecco's Modified Eagle's Medium (DMEM) containing $4.5 \mathrm{~g} / \mathrm{l}$ glucose and L-glutamine. The medium was supplemented with $10 \%$ fetal calf serum (FCS). Cells were cultivated under standard cell culture conditions at $37^{\circ} \mathrm{C}$ in a humidified atmosphere under $5 \% \mathrm{CO} 2$ in an incubator and grown up to about $80 \%$ confluency.

For sub-cultivation medium was removed from the cells, cells were washed twice with sterile Dulbecco's Phosphate Buffered Saline (DPBS), and treated with $0.05 \%$ trypsine-EDTA solution until they had detached from the dish. The enzyme was inhibited by addition of medium in which the cells were subsequently resuspended. When necessary, cells were counted in a Neubauer counting chamber and plated in the desired concentration.

\section{Cryoconservation:}

For cryoconservation eukaryotic cells were detached as described, centrifuged $(1,200$ $\mathrm{x} \mathrm{g}$ for $2 \mathrm{~min}$ at RT), resuspended in freezing medium $\left(1-5 \times 10^{6}\right.$ cells $\left./ \mathrm{ml}\right)$ and aliquoted into storage vials. Vials were placed in an insulated box in a $-80{ }^{\circ} \mathrm{C}$ freezer overnight and finally stored in liquid nitrogen.

- Freezing medium:

$90 \%$ FCS

$10 \%$ dimethyl sulfoxide (DMSO)

Revitalization:

For revitalization cryoconserved cells were quickly thawed in a $37^{\circ} \mathrm{C}$ water bath after being removed from liquid nitrogen. Then, cells were pipetted in pre-warmed medium and centrifuged $(1,200 \times \mathrm{g}$ for $2 \mathrm{~min}$ at RT). The medium was removed from the cells and cells were resuspended in medium and then placed into a flask containing medium.

Mycoplasma test:

Mycoplasma contamination may cause adverse effects, such as changes in metabolism, growth rate, viability, DNA, RNA and protein synthesis, morphology etc., which lead to unreliable experimental results. Therefore, cell cultures were regularly tested for the presence of mycoplasma using Mycoplasma Plus PCR Primer Set according to the manufacturer's instructions. 


\subsubsection{Incubation of cells with fluorescence probes}

\section{Fluorescence conjugates:}

To ensure better attachment of KPL-4 and MDA-MB-231 breast cancer cells to 4-well chambered coverglasses, they were covered with sterile poly-L-lysine solution ( 0.1 $\mathrm{g} / \mathrm{l}$ ). After $3 \mathrm{~h}$ incubation at $37^{\circ} \mathrm{C}$, coverslips were washed several times with sterile PBS, and used for seeding of cells. $48 \mathrm{~h}$ prior to incubation with fluorescence probes cells were seeded at a density of $2 \times 10^{4} \mathrm{KPL}-4$ cells and of $1.2 \times 10^{4}$ MDA-MB-231 cells per well in $500 \mu$ l DMEM. After $48 \mathrm{~h}$ the medium was removed and cells were washed once with PBS. Cells were supplemented with $500 \mu \mathrm{L}$ DMEM and 0.1 M 4-(2hydroxyethyl)-1-piperazineethanesulfonic acid (HEPES) buffer containing $5 \mu \mathrm{g}$ fluorescence conjugates or $0.2 \mathrm{nmol}$ of the free fluorescence dyes (corresponding to the amount of dye in $5 \mu \mathrm{g}$ conjugate with a DP ratio of 6) and incubated for $8 \mathrm{~h}$ at 37 ${ }^{\circ} \mathrm{C}$. In order to analyze signals deriving from membrane bound conjugates, probes were also incubated with the cells for $8 \mathrm{~h}$ at $4{ }^{\circ} \mathrm{C}$ where internalization should be strongly reduced ${ }^{5}$. Afterwards, the medium was removed and the cells were washed several times with PBS to remove unbound substances. Subsequently, cells were incubated with $1 \mu \mathrm{g}$ Hoechst 33342 in $500 \mu \mathrm{l}$ PBS $(2 \mathrm{mg} / \mathrm{l}$ ) for $10 \mathrm{~min}$ in order to stain the nuclei of living cells. Hoechst solution was removed and cells were covered with $500 \mu \mathrm{l}$ PBS and directly used for fluorescence microscopy. The settings used for detection of fluorophores are listed in section 2.2.8.3.

\section{Itrybe-loaded NPs:}

Adherent breast cancer cells were seeded on poly-L-lysine-coated cover slips (treated as described above) in 24-well chambers $48 \mathrm{~h}$ prior to incubation with Itrybe-loaded NPs. KPL-4 cells were seeded at a density of $3 \times 10^{4}$ cells and MDA-MB-231 cells were seeded at a density of $1.8 \times 10^{4}$ cells per well in $500 \mu \mathrm{l}$ DMEM. After $48 \mathrm{~h}$ the medium was removed and cells were washed once with PBS and supplemented with $500 \mu \mathrm{L}$ DMEM and 0.1 M HEPES buffer containing NPs at a concentration of $1 \mathrm{~g} / \mathrm{l}$. The cells were incubated for different time periods ( $30 \mathrm{~min}$ to $5 \mathrm{~h}$ ) at $37^{\circ} \mathrm{C}$ and $4{ }^{\circ} \mathrm{C}$. Afterwards, the medium was removed and the cells were washed 3 times with PBS to remove unbound substances. Subsequently, cells were fixed with $4 \%$ formaldehyde in PBS for 10 min on ice and then washed 3 times with PBS again. The cells were mounted with an antifade reagent supplemented with 4',6-diamidino-2-phenylindole (DAPI) for counter stain of cell nuclei. Samples were allowed to dry for $24 \mathrm{~h}$ at $4{ }^{\circ} \mathrm{C}$ and subsequently used for fluorescence microscopy. The settings used for detection of fluorophores are listed in section 2.2.8.3. 


\subsubsection{Assessment of cell viability}

Effects of NPs on proliferation and viability of cells were quantified via use of WST-1 cell proliferation reagent. This assay is based on the enzymatic cleavage of the tetrazolium salt WST-1[2-(4-Iodophenyl)-3-(4-nitrophenyl)-5-(2,4-disulfophenyl)$2 \mathrm{H}$-tetrazolium] (WST-1) to a water-soluble formazan dye by mitochondrial succinate-tetrazolium reductase which is active in viable cells. Absorbance of the dye is directly proportional to the amount of viable cells.

3T3 mouse fibroblasts were seeded at a density of $5 \times 10^{3}$ cells/well in 96 well plates $24 \mathrm{~h}$ before NP treatment. Cells were incubated with 0.01 to $2 \mathrm{~g} / \mathrm{l}$ of $100 \mathrm{~nm}$ NPs or left untreated in DMEM for $24 \mathrm{~h}$ at $37^{\circ} \mathrm{C}$. To consider the absorbance caused by the Itrybe-loaded NPs itself, prior to addition of WST-1-reagent absorbance was measured in the Wallac 1420 Victor 2 Multilabel Counter at $450 \mathrm{~nm} .10 \mu \mathrm{l}$ of WST-1 ready-to-use solution was added to the wells and after $30 \mathrm{~min}$ of incubation at $37^{\circ} \mathrm{C}$ absorbance was measured again at $450 \mathrm{~nm}$. To obtain only the signals derived from the formazan dye, absorbance measured prior to addition of WST-1 was subtracted from the respective wells measured after incubation with WST-1.

\subsubsection{Cell preparation for animal experiments}

One day before implantation, KPL-4 or MDA-MB-231 cells were sub-cultivated to achieve exponential growth. On the day of implantation cells were trypsinized and washed twice with medium. The cell number was determined as described in 2.2.5.1 and suspension volumes containing $5 \times 10^{6} \mathrm{KPL}-4$ cells or $1 \times 10^{6} \mathrm{MDA}-\mathrm{MB}-231$ cells were pelletized under centrifugation at $1000 \times \mathrm{g}$, the medium was removed, and cells were resuspended in $500 \mu \mathrm{l}$ medium.

\subsubsection{In vivo experiments}

All animal experiments were according to $\S 8$ of the animal protection law in the version of 2006 and all animal protocols were approved by the administration of lower Saxony with the animal experiment number 33.14-42502-04-10/0064.

\subsubsection{Animal models}

Experiments were performed on female athymic nude mice, strain NMRI-Fox1nu/nu. Feeding occurred via pelletized Extrudate 1439 food and acidulated water with a $\mathrm{pH}$ of 2.5. Two weeks before the mice were scanned in the Optix MX2 they received chlorophyll-depleted low fluorescent food, GLP Nafag feed 890 or Regime 210, U8959A01R. The mice were maintained in a sterile environment in special cages with 
filter huts placed in a scantainer. Cages, bedding, and water were autoclaved and the food was gamma irradiated.

\subsubsection{Orthotopic implantation of breast tumor cells in mice}

KPL-4 and MDA-MB-231 breast tumor cells were implanted in the right abdominal mammary fat pad of female nude mice at the age of 6 to 12 weeks. For this purpose mice were anesthetized by intraperitoneal (i.p.) injection of $15 \mathrm{mg} / \mathrm{kg}$ body weight xylazine und $75 \mathrm{mg} / \mathrm{kg}$ body weight ketamine in a $1: 1$ dilution with isotone sodium chloride ( $\mathrm{NaCl}, 0.9 \%$ ) solution using an insulin syringe (Ominifix 40 solo) and a sterican needle $(26 G \times 1 / 2)$. The solution containing the respective amount of tumor cells $\left(5 \times 10^{6} \mathrm{KPL}-4\right.$ cells or $1 \times 10^{6} \mathrm{MDA}-\mathrm{MB}-231$ cells, prepared as described in section 2.2.5.4) was centrifuged, the medium was discarded, and cells were resuspended in a volume of $30 \mu \mathrm{PBS}$ and taken up with an insulin syringe with integrated needle (30G). Anesthetized mice were placed under a laminar flow in a dorsal position and the skin was opened with a small incision in the region over the right abdominal mammary fat pad. Tumor cells were implanted very slowly into the mammary fat pad. The needle was slowly withdrawn after a 1-minute delay. The mammary gland was then returned, and the incision was closed using absorbable surgical suture material, 4/0. Afterwards, mice were placed in cages on a heating plate at $37^{\circ} \mathrm{C}$ for $3 \mathrm{~h}$. In time intervals of three days body weight and general condition of the mice were controlled.

\subsubsection{Application of probes}

Mice were used for in vivo fluorescence imaging experiments 1 to 4 month after tumor implantation when tumors reached sizes of approximately $0.3 \mathrm{~cm}^{3}$. Biodistribution experiments with NPs were carried out on tumor-free nude mice. All fluorescent probes were injected solved in $\mathrm{NaCl}, 0.9 \%$ with an insulin syringe with integrated needle ( $30 \mathrm{G} \times 1 / 2$ ). During injection, the animals were anesthetized with vaporized isoflurane at $0.8-1 \%$ concentration.

The amounts of fluorescence probes injected in mice and the different application methods are listed in Table 4. Before and after probe injection, mice were imaged in the Optix MX2 as described in section 2.2.8.4. 
Table 4. Amount and application methods of fluorescent probes injected in mice

Different amounts of fluorescent Herceptin-conjugates, the respective free fluorescence dyes, or Itrybeloaded NPs were injected in mice, either intravenously (i.v.), subcutaneously (s.c.), or intratumoral (i.t.).

\begin{tabular}{|c|c|c|c|}
\hline probe & amount & volume & application \\
\hline fluorescence conjugates & $25 \mu \mathrm{ggG}$ & $150 \mu \mathrm{l}$ & i.v. \\
\hline free dyes & $0.8 \mathrm{nmol} *$ & $150 \mu \mathrm{l}$ & i.v. \\
\hline fluorescence conjugates & $10 \mu \mathrm{g} \mathrm{IgG}$ & $50 \mu \mathrm{l}$ & s.c. \\
\hline free dyes & $0.1 \mathrm{nmol}$ & $50 \mu \mathrm{l}$ & s.c. \\
\hline $100 \mathrm{~nm}$ NPs & $400 \mu \mathrm{g}$ & $200 \mu \mathrm{l}$. \\
\hline $25 \mathrm{~nm}$ NPs & $400 \mu \mathrm{g}$ & $200 \mu \mathrm{l}$ & i.v. \\
\hline $15 \mathrm{~nm}$ NPs & $1 \mathrm{mg}$ & $200 \mu \mathrm{l}$ & s.c. \\
\hline $15 \mathrm{~nm}$ NPs & $0.8-100 \mu \mathrm{g}$ & $30 \mu \mathrm{l}$ & i.t. \\
\hline $15 \mathrm{~nm}$ NPs & $1.6 \mu \mathrm{g}$ & $30 \mu \mathrm{l}$ & . \\
\hline
\end{tabular}

* Amount corresponds to the amount of dye in the Herceptin-conjugates with a DP ratio of 5.

\subsubsection{Dissection}

At the end of the in vivo experiments mice were sacrificed by cervical dislocation under high concentration of isoflurane anesthesia. The animals were examined macroscopically for noticeable abnormalities and tumor development. Subsequently, tumors were excised and tumor sizes were measured by a caliper. Under the assumption that the tumor shape is ellipsoid the tumor volume was calculated according to the formula $V\left[\mathrm{~cm}^{3}\right]=$ (length $\mathrm{x}$ width $\mathrm{x}$ height) / 2. Furthermore, the abdomen and the thoracic cavity were opened and liver, kidneys, spleen, lung, heart, stomach, and gut were isolated and imaged ex vivo.

\subsubsection{Tumor preparation}

One half of the tumor was stored in a cryotube at $-80^{\circ} \mathrm{C}$ and used for mRNA and/or protein analysis. The other half was fixed with formaldehyde ( $4 \%$ in PBS) over night, embedded in paraffin and used for microscopic examination of tumor tissue. For this purpose, the formalin fixed tumors were placed in Tissue Tek cassettes, rinsed under the water-tap for half an hour and dehydrated and paraffinized in the apparatus for tissue processing over night. 
The following steps were performed:

\begin{tabular}{|c|c|c|}
\hline repetition cycles & time & applied substance \\
\hline three & $1 \mathrm{~h}$ & $75 \%$ ethanol \\
\hline two & $1 \mathrm{~h} 30 \mathrm{~min}$ & $96 \%$ ethanol \\
\hline three & $1 \mathrm{~h} 15 \mathrm{~min}$ & $100 \%$ ethanol \\
\hline two & $2 \mathrm{~h}$ & xylol \\
\hline two & $1 \mathrm{~h}$ & paraffin \\
\hline
\end{tabular}

\subsubsection{Histological examinations of tumor tissue}

\subsubsection{Generation of paraffin sections from tumor tissue}

Sections of the paraffin-fixed tumors were made with the microtome in 2.5 to $5 \mu \mathrm{m}$ thickness. Sections were attached to object slides, and stored at RT until staining.

\subsubsection{Haematoxylin-Eosin (HE) staining of paraffin-embedded}

\section{tumor sections}

Sections were incubated over night at $37^{\circ} \mathrm{C}$ in the incubator to improve attachment of the tissue to the microscope slides. Afterwards the tumor sections were deparaffinized twice with xylol followed by rehydration in decreasing concentrations of ethanol (each for $2 \mathrm{~min}$ ). The Haematoxylin staining of cell nuclei was done for 5 to $10 \mathrm{~min}$ and after washing with water the cell structures were stained with Eosin $\mathrm{G}$ (1: 10 dilution in $\mathrm{H}_{2} \mathrm{O}$ ) for 5 to 10 min. Tumor sections were washed with water and dehydrated with increasing concentrations of ethanol (50\%, $70 \%, 90 \%, 100 \%$ ) and xylol and mounted in hydrophobic mounting medium (DePex). Sections were analyzed under the light microscope.

\subsubsection{CD31 staining}

Paraffin-embedded tumor sections ( $2.5 \mu \mathrm{m}$ thickness) were stained with the antiendothelial cell marker cluster of differentiation 31 (CD31) antibody after being deparaffinized as described in section 2.2.7.2. Prior to antibody treatment, sections were incubated for $20 \mathrm{~min}$ in target retrieval solution at $98{ }^{\circ} \mathrm{C}$ in a steamer. After cooling down, the sections were washed twice with TRIS buffer. The endogenous peroxidase was blocked with $3 \%$ hydrogen peroxid in PBS for $10 \mathrm{~min}$ at RT. Afterwards unspecific binding sites were blocked with commercial blocking buffer from fish for 15 min at RT. Incubation of tissue with anti-mouse CD31 antibody at a 
1:100 dilution was carried out in commercial background reducing antibody diluent over night at $4{ }^{\circ} \mathrm{C}$. Afterwards, the sections were washed twice with TRIS buffer and incubated with the secondary HRP-coupled anti-rat antibody at a 1:200 dilution in TRIS-buffer for $1 \mathrm{~h}$ at RT. Sections were washed twice with TRIS buffer followed by incubation with peroxidase substrate, 3-amino-9-ethylcarbazole (AEC) for $30 \mathrm{~min}$ at RT, and washed twice with water. Staining of cell nuclei was performed by incubation in Haematoxylin for 10-20 sec. The tumor sections were washed with water and mounted with aqueous mounting medium (Aquatex). CD31 staining was analyzed under the light microscope.

- $\quad$ TRIS buffer

50 mM TRIS- $\mathrm{HCl}(\mathrm{pH} 7.6)$

$150 \mathrm{mM} \mathrm{NaCl}$

\subsubsection{Near-infrared (NIR)-microscopic analysis of tumor sections}

Tumors treated in vivo with fluorescent probes:

Paraffin sections $(2.5 \mu \mathrm{m}$ thickness) of tumors of mice treated with Herceptinconjugates in vivo were deparaffinized as described in section 2.2.7.2. and mounted with hydrophobic mounting medium containing DAPI for counterstain of cell nuclei. Samples were allowed to dry for $24 \mathrm{~h}$ at $4{ }^{\circ} \mathrm{C}$. Sections were analyzed under the fluorescence microscope as described in section 2.2.8.3.

\section{Ex vivo treatment with fluorescent probes:}

Paraffin embedded tumor sections ( $5 \mu \mathrm{m}$ thickness) were deparaffinized as described in section 2.2.7.2. The sections were washed 3 times with TBS containing $1 \%$ goat serum and blocked with TBS and $10 \%$ goat serum for $1 \mathrm{~h}$ at RT. Tumor samples were incubated with Itrybe-loaded NPs in TBS and $10 \%$ goat serum for $2 \mathrm{~h}$ at $4{ }^{\circ} \mathrm{C}$. Afterwards, the sections were washed several times with TBST to remove unbound NPs. The cells were mounted with an antifade reagent supplemented with DAPI for counter stain of cell nuclei and allowed to dry for $24 \mathrm{~h}$ at $4{ }^{\circ} \mathrm{C}$. Sections were analyzed under the fluorescence microscope as described in section 2.2.8.3.

\subsubsection{Imaging devices}

\subsubsection{Spectrofluorometer}

Excitation and emission spectra of fluorescence probes and dye-loaded NPs were recorded in a Spectrofluorometer QuantaMaster equipped with a xenon lamp as excitation source and two fluorescence detectors. The solutions were measured in a 
quartz glass precision cuvette with a light pass of $3 \times 3 \mathrm{~mm}$. Spectra were recorded with a step size of $1 \mathrm{~nm}$ and an integration time of $1 \mathrm{sec}$. Wavelengths settings used for recording of spectra are listed in Table 5. Analysis of spectroscopic measurements was performed with the software FeliX32 Analysis 1.0. Measurements are quantified as the mean of signals recorded from both detectors.

Table 5. Settings used for recording of spectra of fluorescent probes

\begin{tabular}{|c|c|c|c|c|}
\hline \multirow{2}{*}{ probe } & \multicolumn{2}{|c|}{ excitation scan } & \multicolumn{2}{c|}{ emission scan } \\
\cline { 2 - 5 } & $\boldsymbol{\lambda}_{\mathbf{e m}}[\mathbf{n m}]$ & $\begin{array}{c}\text { recording } \\
\text { range [nm] }\end{array}$ & $\boldsymbol{\lambda}_{\mathbf{e x}}[\mathbf{n m}]$ & $\begin{array}{c}\text { recording } \\
\text { range [nm] }\end{array}$ \\
\hline $\begin{array}{c}\text { fluorescence } \\
\text { conjugates }\end{array}$ & not analyzed & not analyzed & 635 & $610-750$ \\
\hline Itrybe-loaded NPs & $740-770 *$ & $450-740$ & 590 & $650-875$ \\
\hline
\end{tabular}

$\lambda_{\mathrm{em}}$ : excitation wavelength; $\lambda_{\mathrm{ex}}$ : emission wavelength

* $\lambda_{\text {em }}$ was chosen according to the absorption maximum of the NPs with different sizes, which was $740 \mathrm{~nm}$ for $15 \mathrm{~nm}$ NPs, $750 \mathrm{~nm}$ for the $25 \mathrm{~nm}$ NPs, and $770 \mathrm{~nm}$ for the $100 \mathrm{~nm}$ NPs.

\subsubsection{Odyssey infrared imaging system}

Functionality of bioconjugated Itrybe-loaded NPs was evaluated in the Odyssey infrared imaging system (see section 2.2.3.3 for production of NPs) by exciting the probes with a $685 \mathrm{~nm}$ laser diode. Fluorescence intensity was detected in a raster mode with a resolution of $84 \mu \mathrm{m}$. Analysis of fluorescence measurements was performed with the Odyssey Application Software, Version 2.1.

Average fluorescence intensity was calculated as the sum of the intensity in an area set in the respective well divided by the number of measurement points in the area.

\subsubsection{Fluorescence microscope}

For examination of fluorescence stained human KPL-4 and MDA-MB-231 breast tumor cells, the fluorescence microscope Axiovert 200M equipped with a NIR-sensitive ORCA-ER digital camera was used. Filter settings are illustrated in Table 6.

Table 6. Microscopy filter settings used for detection of fluorophores

\begin{tabular}{|c|c|c|c|}
\hline probe & $\boldsymbol{\lambda}_{\mathbf{e x}}[\mathbf{n m}]$ & $\begin{array}{c}\text { beam splitter } \\
{[\mathbf{n m}]}\end{array}$ & $\boldsymbol{\lambda}_{\mathbf{e m}}[\mathbf{n m}]$ \\
\hline fluorescence probes & $640 \pm 15$ & 660 & $690 \pm 25$ \\
\hline Itrybe-loaded NPs & $620 \pm 30$ & 662 & $809 \pm 45.5$ \\
\hline DAPI, Hoechst & $365 \pm 12.5$ & 395 & $445 \pm 25$ \\
\hline
\end{tabular}


Image generation and processing were performed with the software systems AxioVision Rel.4.6. and Image], respectively.

\subsubsection{In vivo NIRF imager, Optix MX2}

The Optix MX2 system ${ }^{13}$ was used for characterization, quantification and illustration of NIRF probes in vitro and their localization in living animals. The system is a fluorescence imager working in reflectance mode and scanning in a raster scheme with a minimal step size of $0.5 \mathrm{~mm}$. During the scan each raster point is irradiated with 9 ps laser impulses with a repetition rate of $80 \mathrm{MHz}$. The system utilizes a single photon counting detector, measuring the time between an excitation pulse and the detection of the first fluorescence photon. As illustrated in Figure 9, the generated temporal point spread function (TPSF) is a curve representing the distribution of the number of photons coming from a raster scan point on the sample over time. Under the assumption of a mono exponential decay of the measured intensity the fluorescence LT is calculated by linear regression in the slope of the logarithmic TPSF (Figure 9). Thus, the TPSF provides information about the fluorescence intensity and LT of the fluorescence transition.

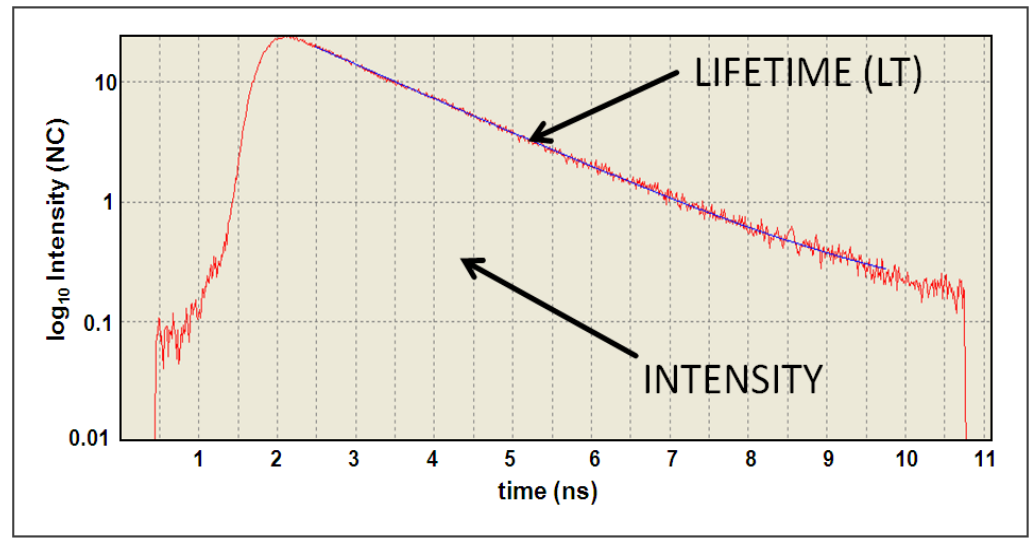

Figure 9. TPSF of one raster scan point

Logarithmic photon time of flight histogram (TPSF) exemplarily shown for one scan point taken from a scan of a mouse injected with a fluorophore. The time between excitation pulse and detection of fluorescence photons is depicted on the $x$-axis. The

fluorescence intensity in a $\log _{10}$ scale is illustrated on the $y$-axis. The fluorescence intensity is determined by the area under the curve. From the decay of the curve the fluorescence LT is calculated.

For scanning mice were placed inside the imaging device on a table preheated to $37^{\circ} \mathrm{C}$ and anesthetized with vaporized isoflurane at 0.8 to $1 \%$ concentration throughout the imaging session.

The excitation lasers $\left(\lambda_{\mathrm{ex}}\right)$ and emission filters $\left(\lambda_{\mathrm{em}}\right)$ selected for detection of the fluorescence conjugates, free fluorescence dyes and Itrybe-loaded NPs are shown in Table 7. 
Table 7. Laser and filter settings used for detection of fluorophores in the Optix MX2

\begin{tabular}{|c|c|c|}
\hline probe & $\boldsymbol{\lambda}_{\mathbf{e x}}[\mathbf{n m}]$ & $\boldsymbol{\lambda}_{\mathbf{e m}}[\mathbf{n m}]$ \\
\hline fluorescence conjugates & 635 & $670 \pm 20$ \\
\hline Itrybe-loaded NPs & $635 / 670 *$ & 700 long pass \\
\hline
\end{tabular}

* Due to the very bright absorption spectrum of Itrybe, excitation was possible with the 635 and $670 \mathrm{~nm}$ laser.

Table 8 shows the measurement time per raster position (integration time) and step size generally used for the different scans in the Optix MX2.

Table 8. Integration time and step sizes used for fluorescence measurements in the Optix MX2

\begin{tabular}{|c|c|c|c|c|c|}
\hline situation: & \multicolumn{2}{|c|}{ in vivo } & \multicolumn{2}{c|}{ ex vivo } & in vitro \\
\hline scan: & tumor & $\begin{array}{c}\text { whole } \\
\text { body }\end{array}$ & tumor & $\begin{array}{c}\text { other } \\
\text { organs }\end{array}$ & solution \\
\hline $\begin{array}{c}\text { integration } \\
\text { time [sec.] }\end{array}$ & 1 & $0.7 / 0.2^{*}$ & 1 & 0.7 & 1 \\
\hline $\begin{array}{c}\text { step size } \\
\text { [mm] }\end{array}$ & 1 & 1.5 & 1 & 1.5 & 0.5 \\
\hline
\end{tabular}

* Biodistribution experiments in healthy mice were performed with an integration time of 0.2 for whole body scans.

The laser power was adapted automatically for optimal detection of the fluorescence yield. Analysis of the fluorescence measurements was performed with the software optiview 1.00 .00 (ART).

The following parameters were calculated for data analysis:

Normalized counts (NC):

The measured fluorescence signals (counts) were normalized for varying laser power and integration times, thus allowing comparison of measurements with different settings.

Average fluorescence intensity:

The average fluorescence intensity over a certain area was determined as the sum of the NC in the area divided by the number of measurement points in the area.

Subtraction of autofluorescence:

In order to eliminate unspecific signals deriving from endogenous particles of the mouse, subtraction of autofluorescence was performed. For this purpose, the average 
fluorescence intensity in a region of the prescan of the mouse (e.g. the tumor area) was calculated and subtracted from the intensity maps after probe application.

Contrast to noise ratio (CNR):

To consider the signal difference from the average intensity over a certain area and those over the background, the contrast to noise ratio (CNR) was calculated. This ratio provides information about the level of signal differences and the affection of the signal by the noise (e. g. electrical- or photon noise). The CNR was calculated as followed:

$$
\text { CNR }=\frac{\text { (average intensity tumor area }- \text { average intensity backround area) }}{\text { standard deviation background area }}
$$

\section{Fluorescence LT:}

Fluorescence LT is the average time an atom stays in the meta-stable state before its transition to the ground state under emission of a fluorescent photon. Thus the LT of the fluorescence transition is the characteristic value of a fluorescent probe. Fluorescence LT measurements enable a differentiation between fluorescence signals originating from the fluorescence probe and unspecific- or autofluorescence.

\section{Average fluorescence LT:}

The average fluorescence LT over a certain area was determined as the sum of the LTs in the area divided by the number of measurement points in the area.

\section{LT goodness of fit:}

For determination of the quality of the measured LT, the LT goodness of fit was calculated. Since, the decay of the fluorscence over a certain point is modeled with a mono exponential decay function, a signal derived from two or more fluorescence sources would no longer show a mono exponential decrease but an overlay of several signal decreases. Thus, the determined LT would arise from two or more fluorescent species leading to a high LT error and a poor accuracy of the LT estimation. A LTcalculation with a LT goodness of fit of $1 \pm 0.2$ is considered to have a high accuracy. 


\section{Results}

\subsection{Characterization of tumor model organisms}

Fluorescent probes evaluated in this study were coupled to Herceptin in order to achieve specific binding of probes to HER2-positive breast tumor cells in vitro and in vivo. Therefore, HER2-positive, KPL-4 ${ }^{101}$ as well as HER2-negative, MDA-MB-231 ${ }^{102}$ tumor models were established. Prior to evaluation of fluorescence probes in vivo, tumor cells were analyzed for HER2 expression levels and their suitability to bind Herceptin-conjugated probes.

\subsubsection{Orthotopic breast tumor models in nude mice}

Tumor-bearing mice were generated by orthotopic implantation of KPL-4 or MDA-MB231 tumor cells in the right abdominal mammary fat pad of female nude mice at the age of 6-12 weeks. Imaging of mice bearing KPL-4 tumors started $8.3 \pm 4.6$ weeks after implantation when the tumors reached a mean size of $0.28 \pm 0.19 \mathrm{~cm}^{3}(\mathrm{n}=$ 24). MDA-MB-231 tumor-bearing mice were scanned after $9.9 \pm 9.2$ weeks, when tumors had a mean size of $0.25 \pm 0.07 \mathrm{~cm}^{3}(n=16)$. In Figure 10, representative KPL-4 (Figure 10A) and MDA-MB-231 (Figure 10B) tumor-bearing mice are shown. Due to the lack of fur in nude mice, the tumor is clearly visible in vivo (Figure $10 A, B$; upper part) and the growth rate can be assessed by caliper measurements easily. Section through the excised tumors show necrotic areas, which have been already developed at the described tumor size in both models (Figure 10A,B; lower part). In addition, for the KPL-4 tumors, a soft consistency at the tumor center was noted (Figure 10A, lower, right picture). No invasive growth and metastases were detected in both tumor types. 


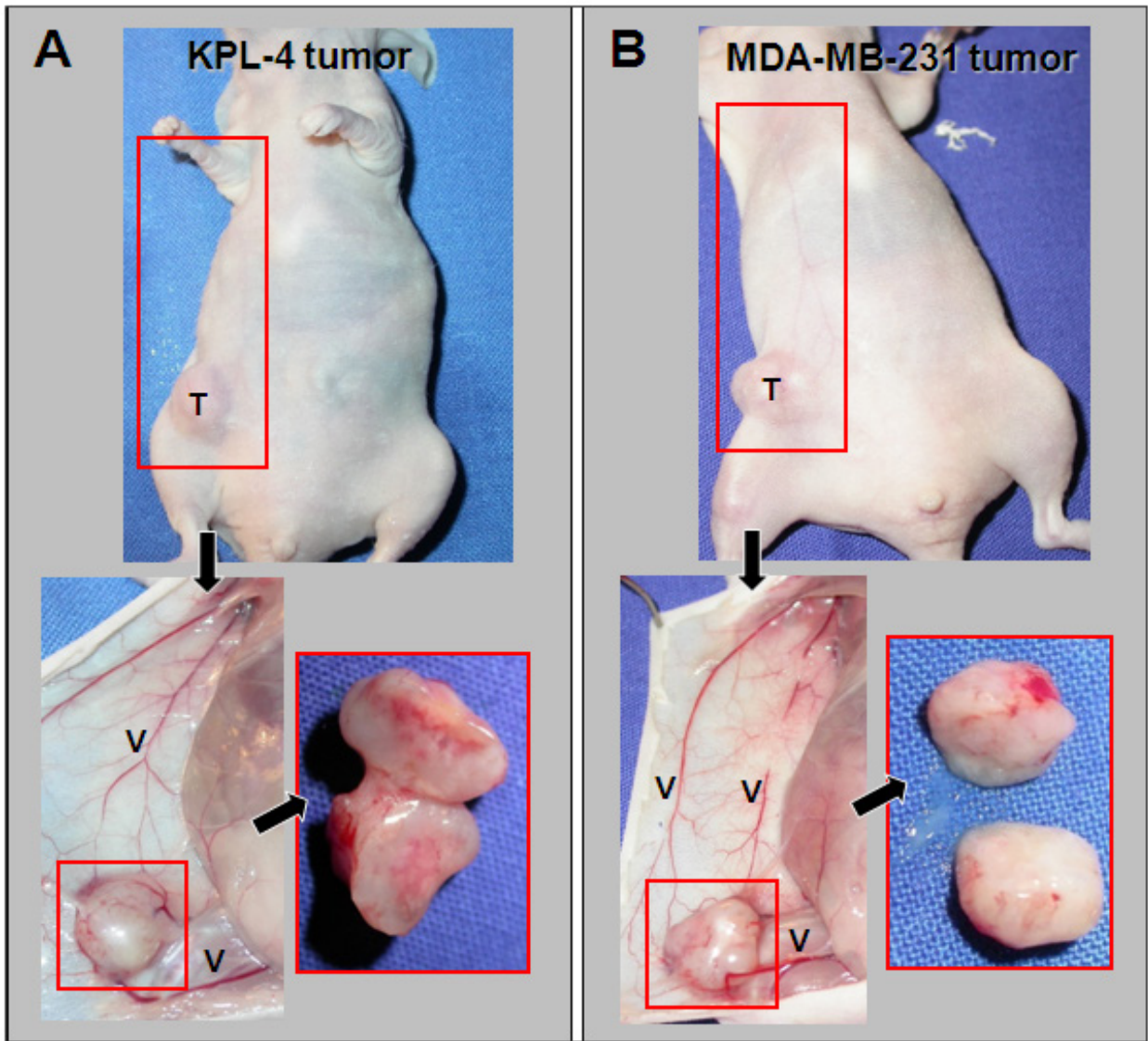

Figure 10. Macroscopic appearance of orthotopic breast tumor xenografts in nude mice

Human tumor cells were implanted orthotopically in the right abdominal mammary fat pad of female nude mice. A,B. Representative ex vivo picture of $(\mathbf{A})$ a nude mouse bearing a $\mathrm{KPL}-4$ tumor $(\mathrm{T})$ with a size of $0.22 \mathrm{~cm}^{3}$ and $(\mathbf{B})$ a nude mouse bearing a MDA-MB-231 tumor $(T)$ with a size of $0.36 \mathrm{~cm}^{3}$. (A,B) Both tumors are supplied by large-diameter blood vessels ( $\mathrm{V}$; lower part, left). A section through the excised tumors (lower part, right) illustrates inhomogeneous tissue with necrotic areas.

Morphology of KPL-4- and MDA-MB-231 tumors was evaluated on sections stained with HE or with a CD-31 antibody for assessment of tumor blood vessels. For both tumor types, KPL-4 (Figure 11A-C; $n=12$ ) and MDA-MB-231 (Figure 11D-F; $n=8$ ) cancerous cells and also vital tumor tissue, such as mammary glands and ducts can be observed (Figure 11A,D; black arrow). Both tumors are supplied by several smalldiameter capillaries (Figure 11C,F; red stars). Towards the tumor border a network of microvessels is observed whereas the vessel density seems to be lower towards the center (data not shown). Moreover, necrotic areas can be seen between vital tumor tissue (Figure $11 A, B, D, E$; white arrow). Here, less necrosis is observed for the MDA-MB-231 tumors (Figure 11A,B; white arrow) that is, in comparison to the KPL-4 tumors (Figure 11D,E) located more towards the tumor center. 


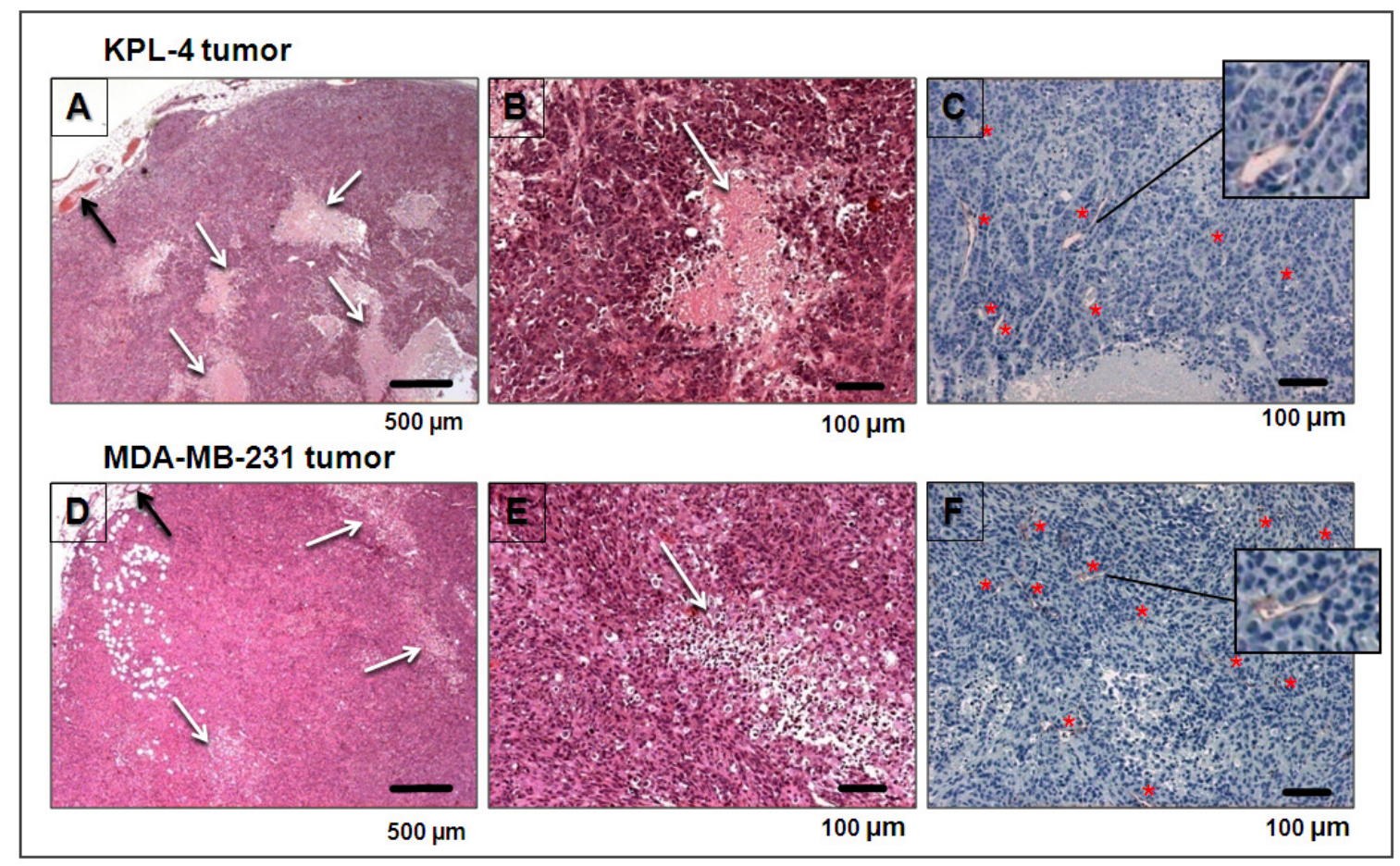

Figure 11. Histology of breast tumor xenografts

A-C. Sections of a representative KPL-4 tumor $(n=12)$. A. HE staining shows several necrotic areas (white arrows) between vital tumor tissue and normal breast tissue (black arrow). B. HE staining at a higher magnification illustrates a necrotic area in detail (white arrow). C. CD31 staining marks endothelial cells (red stars). D-E. Sections of a representative MDA-MB-231 tumor $(n=8)$. D. HE staining shows some necrotic areas (white arrows) between vital tumor tissue (black arrow). E. HE staining at a higher magnification illustrates a necrotic area in detail (white arrow). F. CD31 staining marks endothelial cells (red stars). Images $A, D$. were taken with a $\times 2.5$ objective and images $B, C, E, F$. were taken with a $\times 10$ objective.

Taken together, KPL-4 and MDA-MB-231 tumor cells developed orthotopic breast tumors in nude mice that are partly necrotic but well vascularized.

\subsubsection{HER2 expression in breast tumors}

Tumors used in this study were analyzed for HER2 mRNA expression levels via quantitative real-time PCR. In Figure 12, the normalized expression ratio of HER2 in KPL-4 and MDA-MB-231 cells as well as in a corresponding tumor, are illustrated. High expression ratios were found in a KPL-4 tumor (sample no. 3383) and in KPL-4 cells whereas a MDA-MB-231 tumor (sample no.3450) and MDA-MB-231 cells show a very low HER2 expression. 


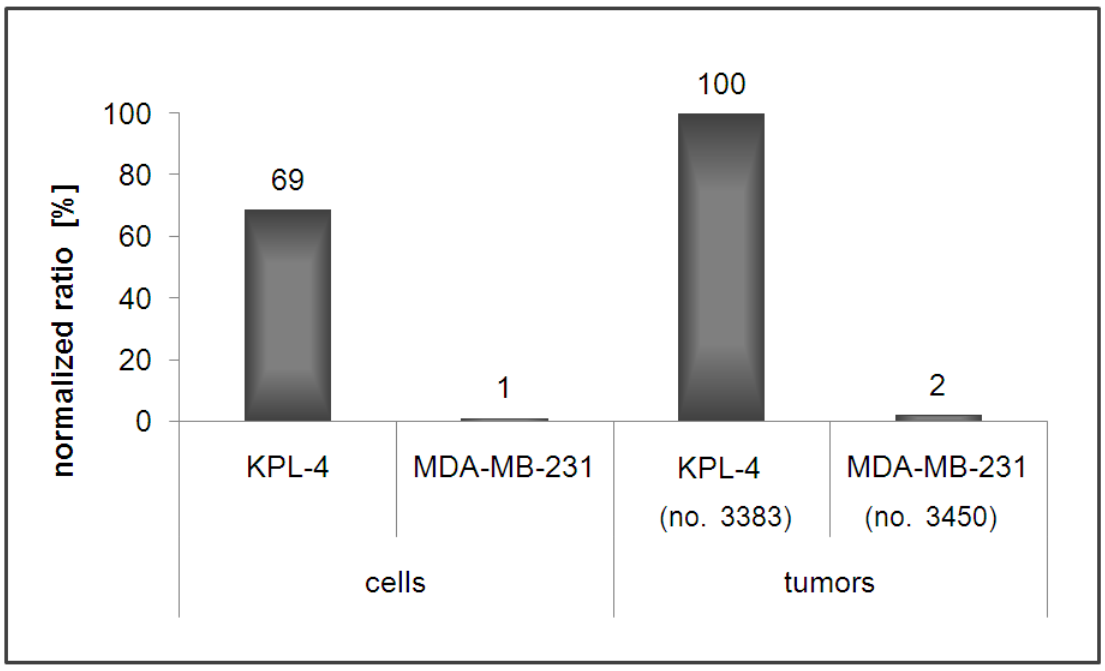

Figure 12. Expression levels of HER2 MRNA in KPL-4 and MDA-MB-231 cell lines and tumors Representative results of a quantitative real-time PCR $(n=2)$. Expression levels of HER2 are normalized to the amount of human transferrin. The highest normalized ratio detected in the KPL-4 tumor (sample no. 3383)

was set to $100 \%$. All measurements were performed in triplicate.

In addition, the expression levels of the HER2 protein were determined. For this purpose, $50 \mu \mathrm{g}$ of total protein amount of KPL-4 and MDA-MB-231 cells as well as of two tumors, from each cell type were separated via SDS-PAGE and analyzed for the presence of HER2 protein by Western blot. As illustrated in Figure 13, KPL-4 cells and -tumors both show high expression of HER2 whereas MDA-MB-231 cells and tumors show a very weak protein band at the size of HER2 (185 kDa) after incubation with the anti-HER2 antibody. Staining for actin revealed that for all samples a comparable amount of protein was used. (The Western blot experiments were performed by Dr. Fernanda Ramos.)

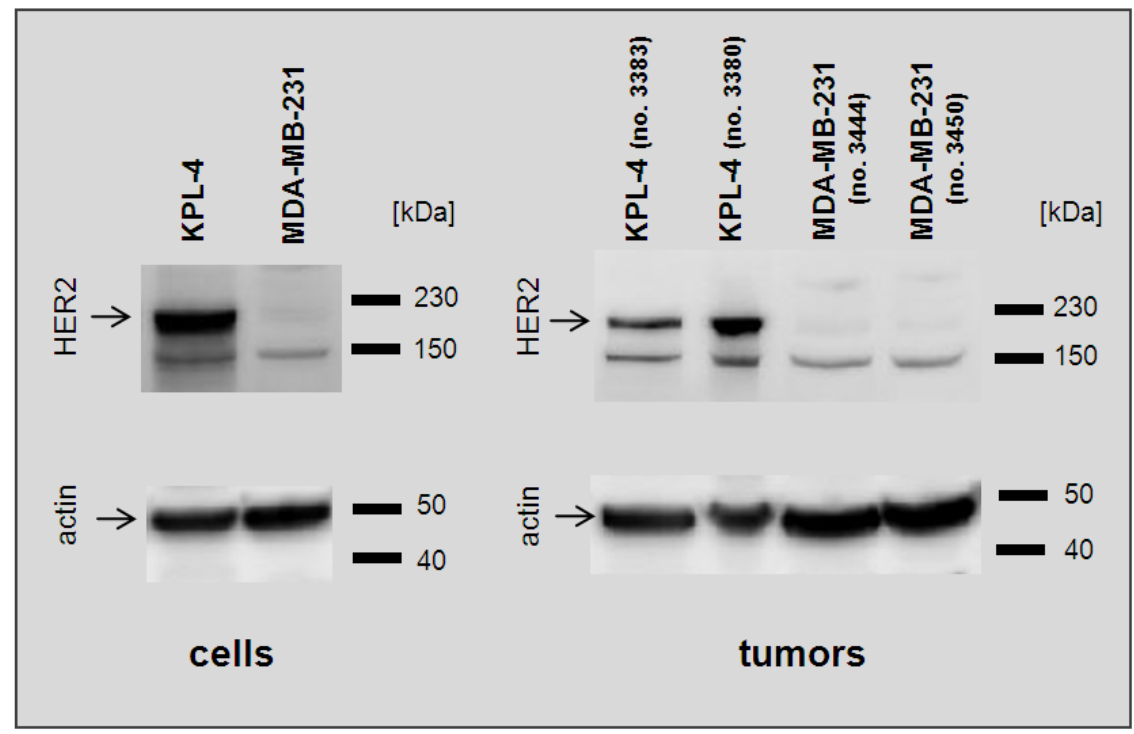

Figure 13. HER2 protein levels in KPL-4 and MDA-MB-231 cell lines and tumors

Western blot analysis revealed that $\mathrm{KPL}-4$ cells (left) and tumors (right; samples no. 3383 and 3380) express HER2 protein showing a band with the size of $185 \quad k D a$ after incubation with an antiHER2 antibody (upper panel). In contrary, almost no staining can be observed in the MDA-MB-231 cells (left) and tumors (right; samples no. 3444 and 3450). Staining of actin reveals comparable amounts of total protein in all samples (lower panel). Representative results of three independent experiments performed by Dr. Fernanda Ramos are shown. 
Taken together, KPL-4 cells as well as tumors developed from these cells express HER2, on the mRNA and protein level. In contrary, MDA-MB-231 cells and tumors show a very low expression of HER2. Therefore, KPL-4 cells (defined as HER2positive) provide an excellent model for the evaluation of Herceptin-conjugated fluorescence probes whereas MDA-MB-231 cells (defined as HER2-negative) were used as negative controls.

\subsubsection{Validation of tumor models for evaluation of fluorescence probes}

To evaluate the suitability of KPL-4 and MDA-MB-231 tumors, to be applied for evaluation of novel fluorescent conjugates in vivo, it is important to ensure that the dye-coupled Herceptin binds to HER2-expressing tumors that developed in mice. In order to enable a high sensitivity of signal detection, fluorescence distribution in the tumor was investigated by using an always-on fluorescent Herceptin-conjugate with high amounts of dyes per antibody. $24 \mathrm{~h}$ after i.v. injection of Alexa-Her at a DP ratio of 3.4, one KPL-4- and one MDA-MB-231 breast tumor were examined under a fluorescence microscope. As shown in Figure 14A, Alexa-Her-derived fluorescence can be detected inside the KPL-4 tumor, located around vital tumor cells. The antibody seems to be not equally distributed in the tumor tissue: Stronger fluorescence is observed in some clustered regions whereas signals in other areas are comparable weak. In contrary, as illustrated in Figure 14B, almost no Herceptin conjugate-derived signals can be detected inside the control MDA-MB-231 tumor. 


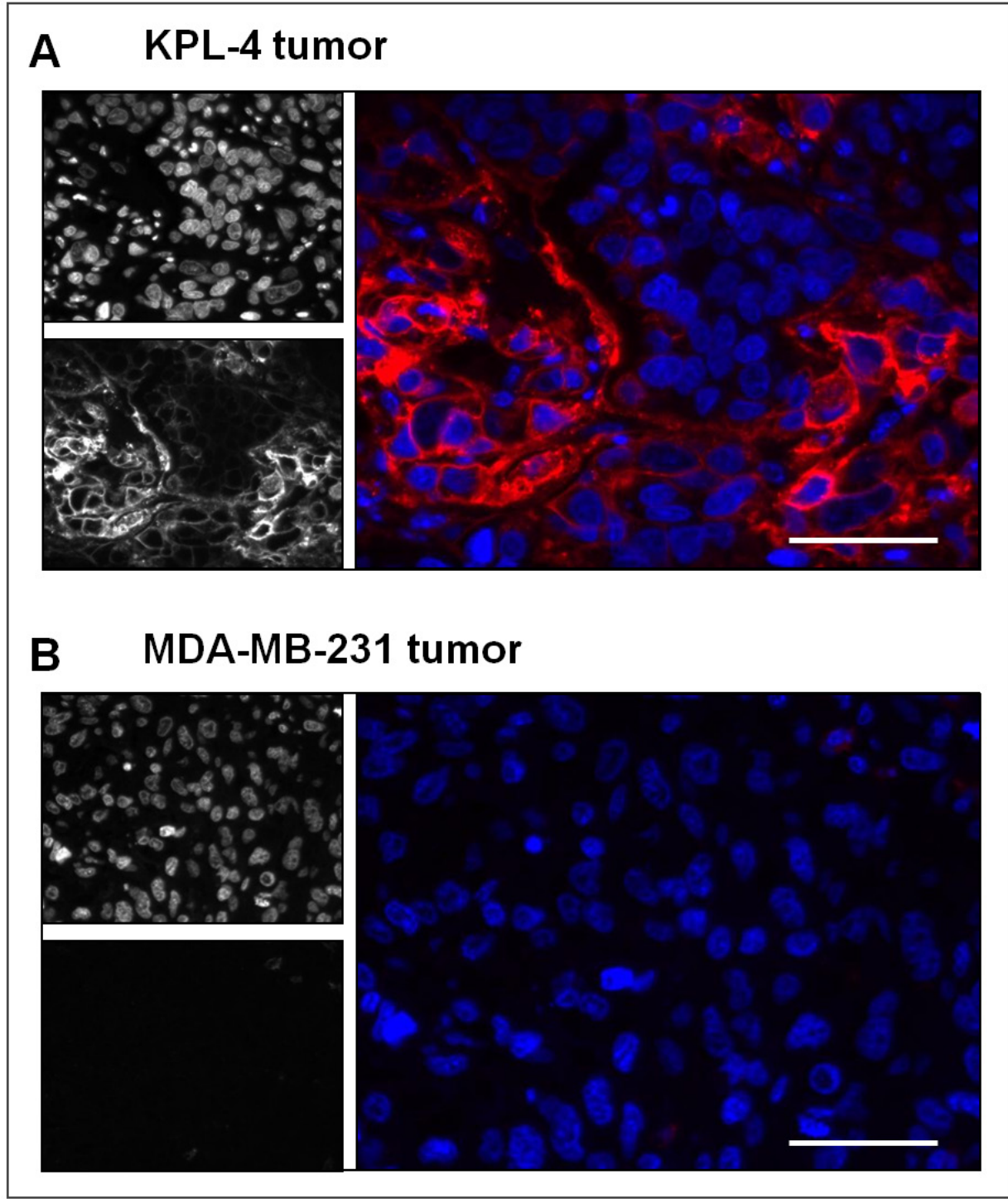

Figure 14. Herceptin-conjugates bind to HER2-expressing tumors in vivo

Sections of paraffin fixed tumors were examined under a fluorescence microscope with a $x 40$ objective. A KPL-4 and a MDA-MB-231 tumor excised from mice $24 \mathrm{~h}$ after receiving i.v. injections of $25 \mu \mathrm{g}$ Alexa-Her at a DP ratio of 3.4 is shown. For each box, on the left upper side, counter stain of cell nuclei with DAPI and on the left lower side, probe-derived signals are illustrated. Merged images of the cell nuclei (blue) and the probe (red) are shown on the right. A. The Herceptin-conjugates are detected inside the KPL-4 tumor. Some tumor areas show stronger accumulation of the conjugates then others. B. In the MDA-MB231 tumor almost no Alexa-Her derived fluorescence is detected. The exposure time for detection of AlexaHer was 300 ms. Bars represent $50 \mu \mathrm{m}$.

Here, we confirmed that breast tumors of HER2-positive, KPL-4 and HER2-negative, MDA-MB-231 cells can be used for the evaluation of Herceptin-conjugated fluorescence probes. 


\subsection{Improvement of tumor detection via pH-sensitive NIRF probes}

There is a high demand for innovative probes with improved fluorescent properties to increase the sensitivity of tumor detection in vivo. In this context, functional fluorescence dyes that selectively activate at the targeted tumor site are promising tools to reduce unspecific signals in the background deriving from circulating and unbound fluorescence probes.

In this part of the work, the functionality and sensitivity of the $\mathrm{pH}$-sensitive tumorspecific probe, $\mathrm{pH}-\mathrm{Her}$ was examined and compared to the always-on fluorescence conjugate Alexa-Her.

\subsection{1. pH-Her conjugates at different DP ratios increase fluorescence in an acidic environment}

In order to investigate the spectroscopic properties of different fluorescence probes with distinct dye to protein (DP) ratios, ranging from statistically 0.5 up to 6.4 dye molecules per Herceptin, fluorescence emission spectra of Herceptin-conjuates were recorded. To illustrate influences of $\mathrm{pH}$ on fluorescence emission, the spectra of all probes were measured in a neutral $\mathrm{pH}$ of 7.5 and an acidic $\mathrm{pH}$ of 5.5.

As illustrated in Figure 15, the shapes of the emission spectra of the pH-sensitive antibody-dye-conjugates, $\mathrm{pH}-\mathrm{Her}$ and the always-on control conjugates, Alexa-Her are comparable to those of the free fluorescence dyes (CypHer5E and Alexa Fluor 647) showing an emission maximum at about $665 \mathrm{~nm}$ for $\mathrm{pH}-\mathrm{Her}$ and about $668 \mathrm{~nm}$ for Alexa-Her (for details see section 2.2.3.1, Figure 7). In an acidic environment ( $\mathrm{pH}$ of $5.5 ; \mathrm{n}=3$ ), all $\mathrm{pH}$-Her probes increase their fluorescence compared to measurements at a neutral $\mathrm{pH}$ (of $7.5 ; \mathrm{n}=3$ ). In contrast, the always-on Alexa-Her probes show no remarkable change in fluorescence in response to a low $\mathrm{pH}(\mathrm{n}=3)$. 

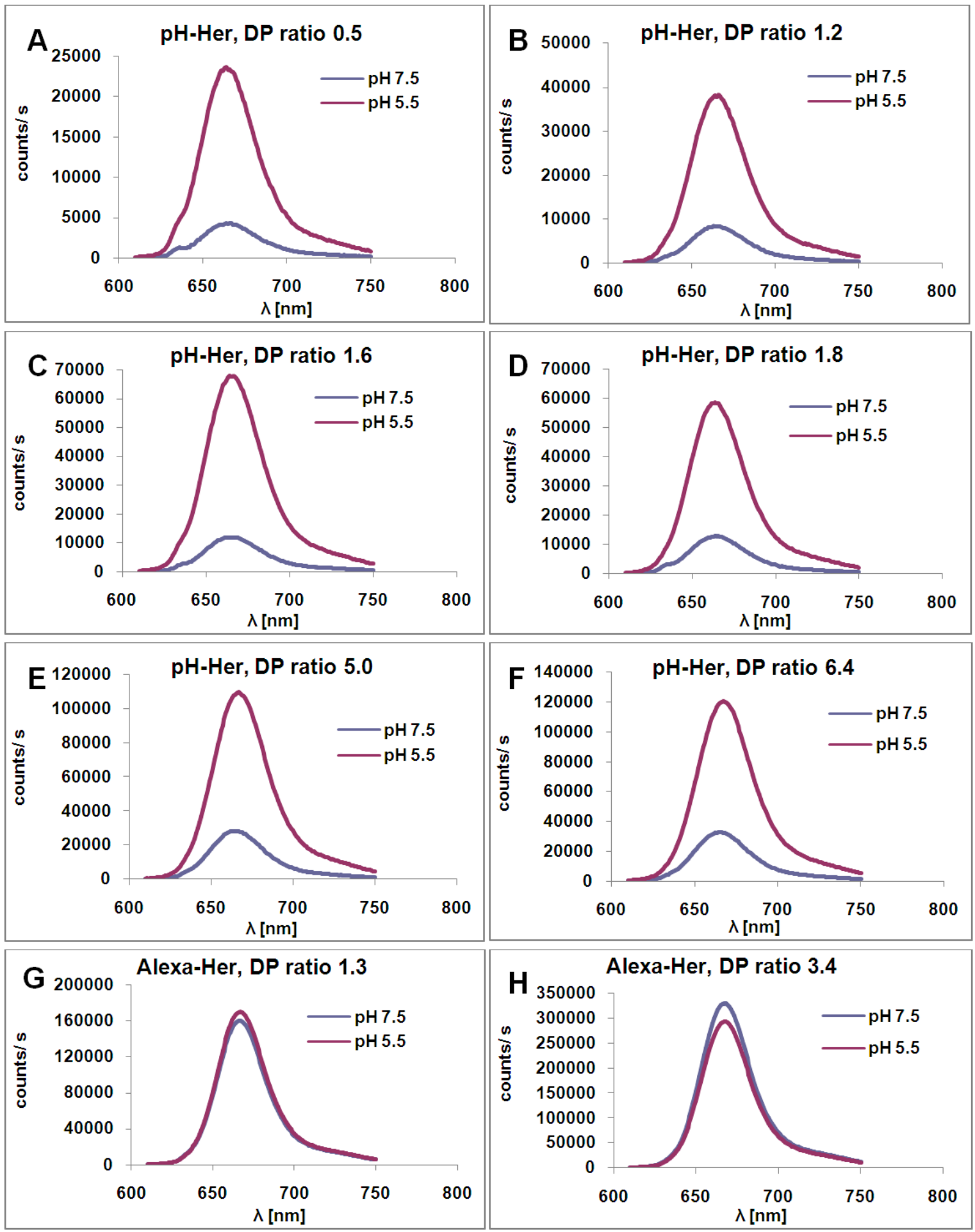

Figure 15. Fluorescence emission spectra of probes at different $\mathbf{p H}$

Representative fluorescence emission spectra of $\mathrm{pH}-\mathrm{Her}$ and Alexa-Her probes recorded from 620 to 750 $\mathrm{nm}$ at $\lambda_{\text {ex }}$ of $635 \mathrm{~nm}$ in a spectrofluorometer $(\mathrm{n}=3$ ). Probes were measured in PBS at a pH of 7.5 (blue curve) and of 5.5 (red curve). A-F. Fluorescence of $\mathrm{pH}-\mathrm{Her}$ with different DP ratios (from 0.5 to 6.4) remarkably increases in a $\mathrm{pH}$ of 5.5 compared to a $\mathrm{pH}$ of 7.5. G, H. No remarkable change in fluorescence can be observed for the Alexa-Her probes at low and high DP ratios (of 1.3 and 3.4) in response to an acidic $\mathrm{pH}$. 
Table 9, summarizes the fluorescence intensities of all probes calculated from the $\lambda_{\mathrm{em}}$ maximum of the spectra illustrated in Figure 15. The pH-Her and Alexa-Her conjugates both show a clear tendency of higher fluorescence with an increase in DP ratio. The later probe also demonstrates much higher intensity when compared to $\mathrm{pH}-\mathrm{Her}$ at comparable DP ratios. For example, the mean intensity of $\mathrm{pH}-\mathrm{Her}$ with a DP ratio of 1.2 at a pH of 5.5 is 44,446 counts/s whereas Alexa-Her with a DP ratio of 1.3 has an almost 4 -fold higher mean fluorescence of 169,582 counts/s, under the same experimental conditions. $\mathrm{pH}-\mathrm{Her}$ conjugates reveal a slightly less $\mathrm{pH}$-sensitivity with increasing DP ratios. For example, pH-Her with a DP ratio of 5.0 shows a 3.9fold increase in fluorescence when measured in a neutral $\mathrm{pH}$ in correspondence to a $\mathrm{pH}$ of 5.5 (factor). In comparison, $\mathrm{pH}-\mathrm{Her}$ with a DP ratio of 1.6 shows the highest increase in fluorescence with a factor of 5.6. In contrast, both Alexa-Her conjugates do almost not change fluorescence in a low $\mathrm{pH}$.

\section{Table 9. Fluorescence intensities of Herceptin-conjugates in dependence of pH}

Fluorescence of $0.07 \mathrm{~g} / \mathrm{l}$ conjugates was recorded in a spectrofluorometer at $\lambda_{\mathrm{em}}$ of $665 \mathrm{~nm}$ for $\mathrm{pH}-\mathrm{Her}$ and $668 \mathrm{~nm}$ for Alexa-Her. Measurements were performed in PBS at a pH of either 7.5 or 5.5. The fluorescence counts/s of three independently performed experiments (1 to 3 ) as well as the mean values are illustrated. The $\mathrm{pH}$-sensitivity of the conjugates is shown as the factor of the mean fluorescence at a $\mathrm{pH}$ of 5.5 divided by the mean fluorescence at $\mathrm{pH} 7.5$.

\section{$\mathrm{pH}-\mathrm{Her}$ conjugates}

\begin{tabular}{c|c|c|c|} 
DP 0.5 & $\begin{array}{c}\mathbf{p H ~ 7 . 5} \\
\text { [counts/s] }\end{array}$ & & $\begin{array}{c}\mathbf{p H ~ 5 . 5} \\
\text { [counts/s] }\end{array}$ \\
\cline { 1 - 2 } & & & 23,624 \\
2 & 3,311 & & 24,161 \\
3 & 5,202 & & 23,457 \\
\hline \hline mean & $\mathbf{4 , 3 1 9}$ & & $\mathbf{2 3 , 7 4 7}$ \\
& \pm 879 & & \pm 368 \\
\hline factor & \multicolumn{3}{|c}{$\mathbf{5 . 5}$} \\
\hline
\end{tabular}

\begin{tabular}{c|c|c|c|} 
DP 1.2 & $\begin{array}{c}\text { pH 7.5 } \\
\text { [counts/s] }\end{array}$ & & $\begin{array}{c}\text { pH 5.5 } \\
\text { [counts/s] }\end{array}$ \\
\cline { 2 - 2 } 2 & 8,415 & & 38,205 \\
2 & 9,186 & & 52,740 \\
3 & 9,048 & & 42,393 \\
\hline \hline mean & $\mathbf{8 , 8 8 3}$ & & $\mathbf{4 4 , 4 4 6}$ \\
& \pm 411 & & $\pm 7,482$ \\
\hline factor & \multicolumn{3}{|c}{$\mathbf{5 . 0}$} \\
\hline
\end{tabular}

\begin{tabular}{c|c|c|c|} 
DP 1.6 & $\begin{array}{c}\mathbf{p H ~ 7 . 5} \\
\text { [counts/s] }\end{array}$ & & $\begin{array}{c}\mathbf{p H ~ 5 . 5} \\
\text { [counts/s] }\end{array}$ \\
& & & 67,832 \\
2 & 11,890 & & 59,381 \\
3 & 9,751 & & 51,224 \\
\hline mean & $\mathbf{1 0 , 6 8 4} \pm$ & & $\mathbf{5 9 , 4 7 9}$ \\
& 1,095 & & $\pm 8,305$ \\
\hline factor & \multicolumn{3}{|c}{$\mathbf{5 . 6}$} \\
\hline
\end{tabular}

\begin{tabular}{c|c|c|c|} 
DP 1.8 & $\begin{array}{c}\text { pH 7.5 } \\
\text { [counts/s] }\end{array}$ & & $\begin{array}{c}\mathbf{p H ~ 5 . 5} \\
\text { [counts/s] }\end{array}$ \\
2 & 12,963 & & 58,480 \\
3 & 11,534 & & 56,458 \\
mean & 13,136 & & 52,796 \\
\cline { 1 - 1 } & $\mathbf{1 2 , 5 4 4} \pm$ & & $\mathbf{5 5 , 9 1 2}$ \\
& 879 & & $\pm 2,881$ \\
\hline factor & \multicolumn{3}{|c}{$\mathbf{4 . 5}$} \\
\hline
\end{tabular}


pH-Her conjugates

\begin{tabular}{c|c|c|c|} 
DP 5.0 & $\begin{array}{c}\text { pH 7.5 } \\
\text { [counts/s] }\end{array}$ & & $\begin{array}{c}\text { pH 5.5 } \\
\text { [counts/s] }\end{array}$ \\
& 28,155 & & 109,445 \\
2 & 23,283 & & 97,068 \\
3 & 21,818 & & 80,698 \\
mean & $\mathbf{2 4 , 4 1 8 \pm}$ & & $\mathbf{9 5 , 7 3 7}$ \\
& 3,318 & & $\pm 14,419$ \\
\hline factor & \multicolumn{3}{|c}{$\mathbf{3 . 9}$} \\
\hline
\end{tabular}

\begin{tabular}{|c|c|c|}
\hline DP 6.4 & $\begin{array}{c}\text { pH } 7.5 \\
\text { [counts/s] }\end{array}$ & $\begin{array}{c}\text { pH } 5.5 \\
{[\text { counts/s] }}\end{array}$ \\
\hline 1 & 32,622 & 120,354 \\
\hline 2 & 40,924 & 174,290 \\
\hline 3 & 20,990 & 82,792 \\
\hline mean & $\begin{array}{c}\mathbf{3 1 , 5 1 2} \pm \\
10,013\end{array}$ & $\begin{array}{r}125,812 \\
\pm 45,992\end{array}$ \\
\hline \multicolumn{3}{|c|}{ factor $\quad 4.0$} \\
\hline
\end{tabular}

\section{Alexa-Her conjugates}

\begin{tabular}{|c|c|c|}
\hline DP 1.3 & $\begin{array}{c}\text { pH } 7.5 \\
\text { [counts/s] }\end{array}$ & $\begin{array}{c}\mathrm{pH} 5.5 \\
\text { [counts/s] }\end{array}$ \\
\hline 1 & 157,824 & 152,775 \\
\hline 2 & 152,639 & 163,651 \\
\hline 3 & 169,503 & 192,321 \\
\hline mean & $\begin{array}{c}159,988 \pm \\
8,638\end{array}$ & $\begin{array}{r}169,582 \\
\pm 20,430\end{array}$ \\
\hline factor & \multicolumn{2}{|c|}{1.1} \\
\hline
\end{tabular}

\begin{tabular}{|c|c|c|}
\hline DP 3.4 & $\begin{array}{c}\text { pH } 7.5 \\
\text { [counts/s] }\end{array}$ & $\begin{array}{c}\text { pH } 5.5 \\
{[\text { counts/s] }}\end{array}$ \\
\hline 1 & 317,535 & 264,915 \\
\hline 2 & 332,074 & 302,574 \\
\hline 3 & 338,816 & 310,969 \\
\hline mean & $\begin{array}{c}\mathbf{3 2 9}, \mathbf{4 7 5} \pm \\
10,876\end{array}$ & $\begin{array}{r}292,819 \\
\pm 24,528\end{array}$ \\
\hline factor & \multicolumn{2}{|c|}{0.9} \\
\hline
\end{tabular}

After spectroscopic characterization, two $\mathrm{pH}-\mathrm{Her}$ conjugates with distinct properties were chosen for further analysis: $\mathrm{pH}-\mathrm{Her}$ with a low DP ratio of $1.6(\mathrm{pH}-\mathrm{Her}$ DP 1.6) due to its high $\mathrm{pH}$-sensitivity (5.6-fold increase in fluorescence from $\mathrm{pH} 7.5$ to $\mathrm{pH}$ 5.5) and $\mathrm{pH}-\mathrm{Her}$ with a high DP ratio of 5.0 (pH-Her DP 5.0) due to its relatively high fluorescence intensity $(96,000$ counts of $0.07 \mathrm{~g} / \mathrm{l}$ probe at a $\mathrm{pH}$ of 5.5$)$. As controls, the always-on conjugates Alexa-Her with a DP ratio of 1.3 (Alexa-Her DP 1.3) and Alexa-Her with a DP ratio of 3.4 (Alexa-Her DP 3.4) were used for further experiments in order to have probes with low and high DP ratios, comparable to the selected $\mathrm{pH}-\mathrm{Her}$ probes.

\subsubsection{LTs of fluorescence probes are influenced by the environment}

Due to its comparable high pH-sensitivity, pH-Her DP 1.6 was applied for LT-gated analysis in vivo. Therefore, LTs of pH-Her DP 1.6 and of the control conjugate AlexaHer DP 1.3 were analyzed in detail and compared to LTs of the respective free fluorescence dyes $\mathrm{pH}-\mathrm{OH}$ and Alexa-OH. 
The influence of the environment on the LT of pH-sensitive- and control-probes was analyzed by examination of the probes under different conditions that are known to influence LTs, like $\mathrm{pH}$ ( $\mathrm{pH}$ of 7.5 and 5.5) or presence of proteins (PBS/BSA $5 \%)^{2,108,109}$. Here, BSA was chosen as serum albumin presents the main plasma protein $(60 \%)$ in humans ${ }^{110}$. In addition, LTs were also determined under in vivo conditions after s.c. injection of probes in mice. The mean LT of all measurements for each probe was defined as the predicted LT and used for probe identification in in vivo LT-imaging.

As summarized in Table 10, pH-Her DP 1.6 has a mean LT of 1.3 ns and shows low variation upon changes in $\mathrm{pH}$, in the presence of proteins, or after s.c. injection. Alexa-Her DP 1.3 has a mean LT of 1.5 ns and reveals slightly longer LTs in the presence of proteins (BSA). The respective free hydrolyzed fluorophores $\mathrm{pH}-\mathrm{OH}$ and Alexa-OH, both have shorter mean LTs (of 1.1 and $1.3 \mathrm{~ns}$ ) compared to the corresponding Herceptin-conjugated flurophores, $\mathrm{pH}-\mathrm{Her}$ and Alexa-Her. Moreover, LTs of both free dyes increase in the presence of proteins, especially at a low pH as well as after s.c. injection in mice.

\section{Table 10. Determination of the probes' LT under different conditions}

LTs of $\mathrm{pH}$-sensitive- and control-probes were determined in PBS and PBS/BSA $5 \%$ with a pH of 7.5 and 5.5 , respectively as well as after s.c. injection in mice. Measurements were performed in triplicates in the Optix MX2. The mean LT of all measurements for each probe was defined as the predicted LT.

\begin{tabular}{c|ccc}
$\begin{array}{c}\text { pH-Her } \\
\text { DP 1.6 }\end{array}$ & pH 7.5 & LT [ns] & pH 5.5 \\
\hline PBS & $1.25 \pm 0.01$ & $1.26 \pm 0.01$ \\
PBS/BSA 5\% & $1.29 \pm 0.03$ & $1.33 \pm 0.01$ \\
in vivo s.c. & & $1.23 \pm 0.01$ \\
\hline predicted $L T$ & \multicolumn{3}{|c}{$\mathbf{1 . 3}$ ns }
\end{tabular}

\begin{tabular}{c|ccc} 
pH-OH & \multicolumn{3}{|c}{ LT [ns] } \\
& $\mathbf{p H ~ 7 . 5}$ & $\mathbf{p H ~ 5 . 5}$ \\
\hline PBS & $0.81 \pm 0.01$ & $0.83 \pm 0.00$ \\
PBS/BSA 5\% & $1.00 \pm 0.01$ & $1.55 \pm 0.02$ \\
in vivo s.c. & \multicolumn{3}{|c}{$1.14 \pm 0.02$} \\
\hline predicted $L T$ & \multicolumn{3}{|c}{$\mathbf{1 . 1}$ ns }
\end{tabular}




\begin{tabular}{c|ccc}
$\begin{array}{c}\text { Alexa-Her } \\
\text { DP 1.3 }\end{array}$ & pH 7.5 & LT [ns] & pH 5.5 \\
\hline \hline PBS & $1.43 \pm 0.03$ & $1.44 \pm 0.03$ \\
PBS/BSA 5\% & $1.53 \pm 0.02$ & $1.63 \pm 0.07$ \\
in vivo s.c. & & $1.36 \pm 0.01$ \\
\hline predicted $L T$ & & $\mathbf{1 . 5} \mathbf{n s}$
\end{tabular}

\begin{tabular}{c|ccc} 
Alexa-OH & \multicolumn{3}{|c}{ LT [ns] } \\
& pH 7.5 & pH 5.5 \\
\hline PBS & $1.17 \pm 0.00$ & $1.14 \pm 0.00$ \\
PBS/BSA 5\% & $1.20 \pm 0.02$ & $1.57 \pm 0.02$ \\
in vivo s.c. & & $1.27 \pm 0.02$ \\
\hline predicted $L T$ & \multicolumn{3}{|c}{$\mathbf{1 . 3}$ ns }
\end{tabular}

Taken together, the influences of a changing environment on the probes' LTs are relatively small. However, the LTs of all probes show a tendency to increase in the presence of proteins. This increase is most prominent for the free dyes measured in BSA-containing solution with a low pH. Furthermore, probes' LTs become longer for both dyes after their conjugation to Herceptin.

\subsection{3. $\mathrm{pH}-s e n s i t i v e$ conjugates are functional in vitro}

In order to test the functionality of the $\mathrm{pH}$-sensitive probes of low and high DP ratios in vitro, fluorescence microscopy with HER2-positive (KPL-4) and HER2-negative (MDA-MB-231) breast cancer cells was performed after incubation with different probes. Herceptin-conjugates are known to internalize and localize in the acidic endosomes and lysosomes within $8 \mathrm{~h}$ of incubation at $37^{\circ} \mathrm{C}^{7,38,92}$ whereas at $4^{\circ} \mathrm{C}$ internalization is strongly reduced ${ }^{5}$. Therefore, cells were incubated with Herceptinconjugates for $8 \mathrm{~h}$ at $37{ }^{\circ} \mathrm{C}$ and at $4^{\circ} \mathrm{C}$ as a control, in order to measure only fluorescence of membrane bound probes, when located in an approximately neutral $\mathrm{pH}$.

The $\mathrm{pH}$-sensitive probe, $\mathrm{pH}-\mathrm{Her}$ DP 1.6 shows fluorescence only after receptormediated internalization into KPL-4 cells (Figure 16A) whereas no signals are detected of membrane bound $\mathrm{pH}-\mathrm{Her}$ (Figure 16B). In contrast, the always-on probe Alexa-Her DP 1.3, shows fluorescence after internalization (Figure $16 \mathrm{C}$ ) as well as when bound to the cell membrane (Figure 16C,D). No fluorescence signals are detected on HER2-negative MDA-MB-231 cells after incubation with all probes (Figure 
$16 \mathrm{E}-\mathrm{H}$ ) at $37^{\circ} \mathrm{C}$ and $4^{\circ} \mathrm{C}$ showing that no unspecific binding and/or internalization occurred.

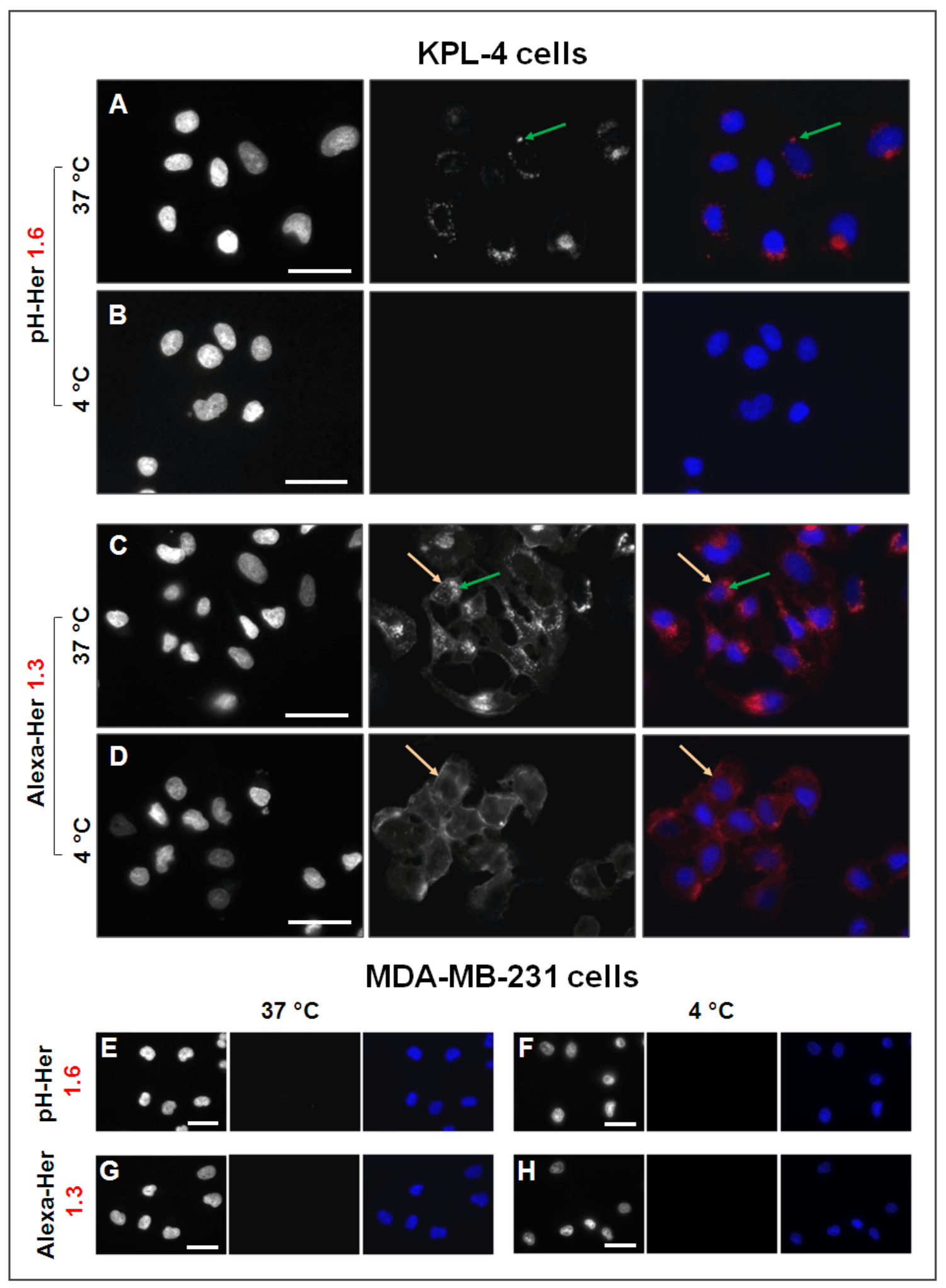

Figure 16. (legend see next page) 
Figure 16. In vitro fluorescence microscopy demonstrates functionality of pH-sensitive probes with a low DP ratio

Cells grown on culture slides were examined under a fluorescence microscope with an $x 40$ objective. For this purpose breast cancer cells were seeded on chambered coverglasses and incubated for $8 \mathrm{~h}$ with the fluorescent probes $\mathrm{pH}-\mathrm{Her}$ or Alexa-Her $(5 \mu \mathrm{g} \mathrm{IgG})$ with a low DP ratio. On the left, counter stain of cell nuclei with Hoechst 33342, in the middle, probe-derived signals, and on the right, merged images of the cell nuclei (blue) and the probe (red) are illustrated. For detection of pH-Her an exposure time of $1500 \mathrm{~ms}$ and for detection of Alexa-Her an exposure time of 200 ms was used. A. When incubated with KPL-4 cells at $37^{\circ} \mathrm{C}$, pH-Her DP 1.6 shows fluorescence only after receptor-mediated internalization (green arrow). B. No signals from the membrane-bound probes are observed, as can be seen by incubation of cells at $4{ }^{\circ} \mathrm{C}$. C,D. In contrast, Alexa-Her DP 1.3 shows fluorescence from the internalized probe (green arrow) but also membrane-derived fluorescence after $8 \mathrm{~h}$ of incubation at (C) $37^{\circ} \mathrm{C}$ and at (D) $4^{\circ} \mathrm{C}$ (yellow arrow). E-H. No fluorescence signals can be detected after $8 \mathrm{~h}$ incubation of MDA-MB-231 cells with pH-Her at (E) $37^{\circ} \mathrm{C}$ or (F) $4{ }^{\circ} \mathrm{C}$ as well as after incubation with Alexa-Her at (G) $37^{\circ} \mathrm{C}$ and $(\mathbf{H}) 4^{\circ} \mathrm{C}$. Representative images of three independently performed experiments are presented. Bars represent $50 \mu \mathrm{m}$.

Incubation of breast cancer cells with $\mathrm{pH}-\mathrm{Her}$ and Alexa-Her conjugates of high DP ratios (Figure 17) revealed similar results as the cell experiments performed with probes with low DP ratios (Figure 16). In KPL-4 cells pH-Her DP 5.0 shows fluorescence only after internalization (Figure 17A) but not when bound to the membrane (Figure 17B) whereas fluorescence of Alexa-Her DP 3.4 is independent on internalization (Figure 17C,D). MDA-MB-231 cells show no fluorescence signals upon incubation with probes with high DP ratios (Figure 17E-H).

In order to prove that fluorescence signals on the KPL-4 cells after incubation with $\mathrm{pH}-\mathrm{Her}$ and Alexa-Her conjugates (Figure 16 and 17) do derive from specifically membrane-bound or internalized Herceptin, control experiments with the free fluorophores were carried out. No fluorescence signals are detected on HER2-positive $\mathrm{KPL}-4$ cells after incubation with $\mathrm{pH}-\mathrm{OH}$ neither at $37^{\circ} \mathrm{C}$ nor at $4{ }^{\circ} \mathrm{C}$ (Figure $18 \mathrm{~A}, \mathrm{~B}$ ) or with Alexa-OH at $4^{\circ} \mathrm{C}$ or at $37^{\circ} \mathrm{C}$ (Figure 18C,D). MDA-MB-231 cells also show no detectable fluorescence when incubated with $\mathrm{pH}-\mathrm{OH}$ or Alexa-OH (Figure $18 \mathrm{E}-\mathrm{H}$ ). 


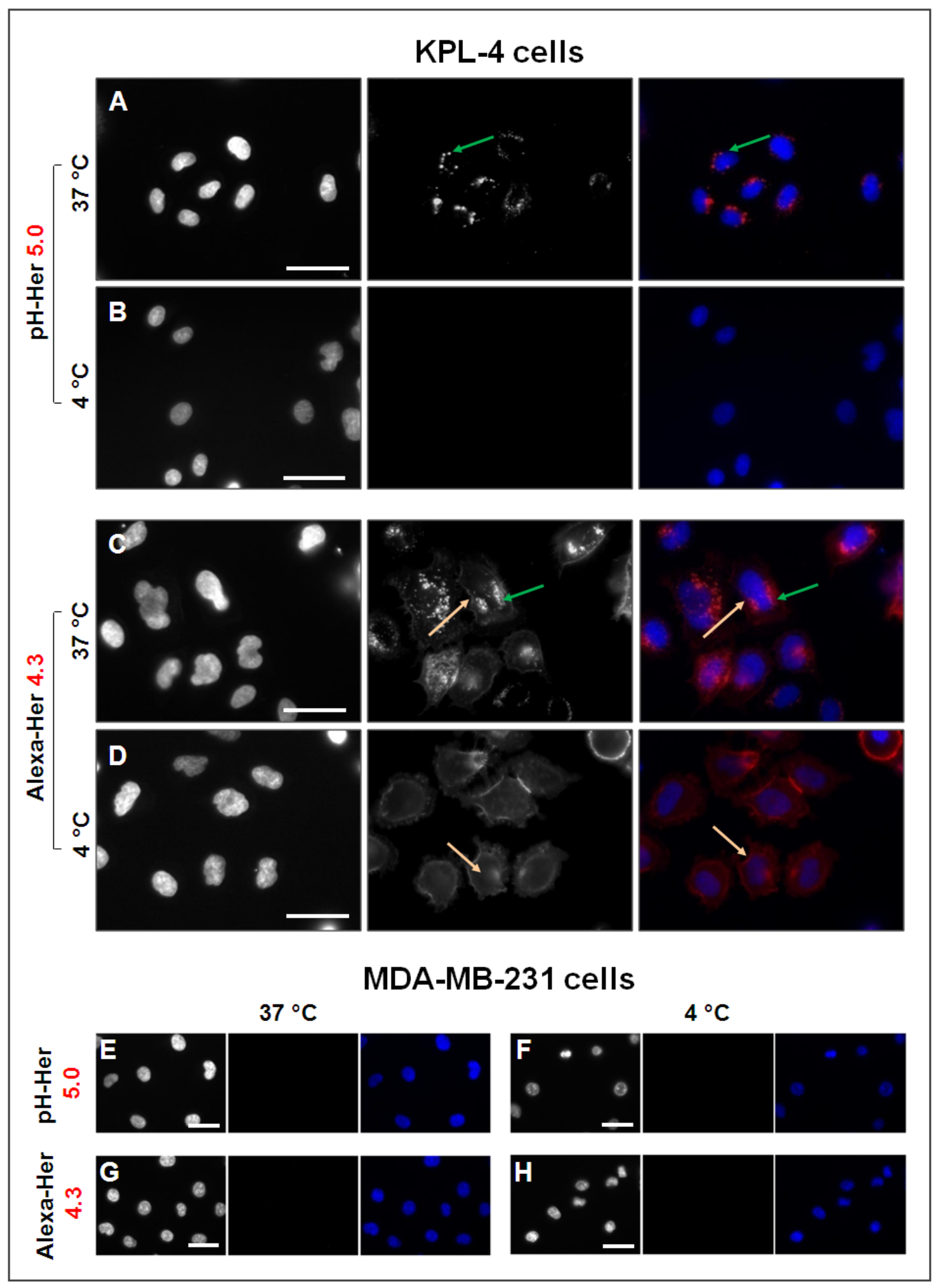

Figure 17. (legend see next page) 
Figure 17. In vitro fluorescence microscopy demonstrates functionality of pH-sensitive probes with a high DP ratio

Cells grown on culture slides were examined under a fluorescence microscope with an $x 40$ objective. For this purpose cells were incubated for $8 \mathrm{~h}$ with $\mathrm{pH}-\mathrm{Her}$ or Alexa-Her fluorescent probes ( $5 \mu \mathrm{g}$ IgG) with a high DP ratio. On the left, counter stain of cell nuclei with Hoechst 33342, in the middle, probe-derived signals, and on the right, merged images of the cell nuclei (blue) and the probe (red) are illustrated. For detection of $\mathrm{pH}-\mathrm{Her}$ an exposure time of $1000 \mathrm{~ms}$ and for detection of Alexa-Her an exposure time of 100 $\mathrm{ms}$ was used. A. When incubated with $\mathrm{KPL}-4$ cells at $37{ }^{\circ} \mathrm{C}, \mathrm{pH}-\mathrm{Her} \mathrm{DP} 5.0$ shows fluorescence only after receptor-mediated internalization (green arrow). B. No signals from the membrane-bound probes are observed at $4{ }^{\circ} \mathrm{C}$. C,D. Alexa-Her DP 3.4 shows fluorescence from the internalized probe (green arrow) but also membrane-derived signals after $8 \mathrm{~h}$ of incubation at (C) $37^{\circ} \mathrm{C}$ and at (D) $4{ }^{\circ} \mathrm{C}$ (yellow arrow). E-H. No fluorescence signals can be detected after $8 \mathrm{~h}$ incubation of MDA-MB-231 cells with pH-Her at (E) $37^{\circ} \mathrm{C}$ or (F) $4{ }^{\circ} \mathrm{C}$ as well as after incubation with Alexa-Her at (G) $37^{\circ} \mathrm{C}$ and $(\mathbf{H}) 4^{\circ} \mathrm{C}$. Representative results of three independently performed experiments are shown. Bars represent $50 \mu \mathrm{m}$.

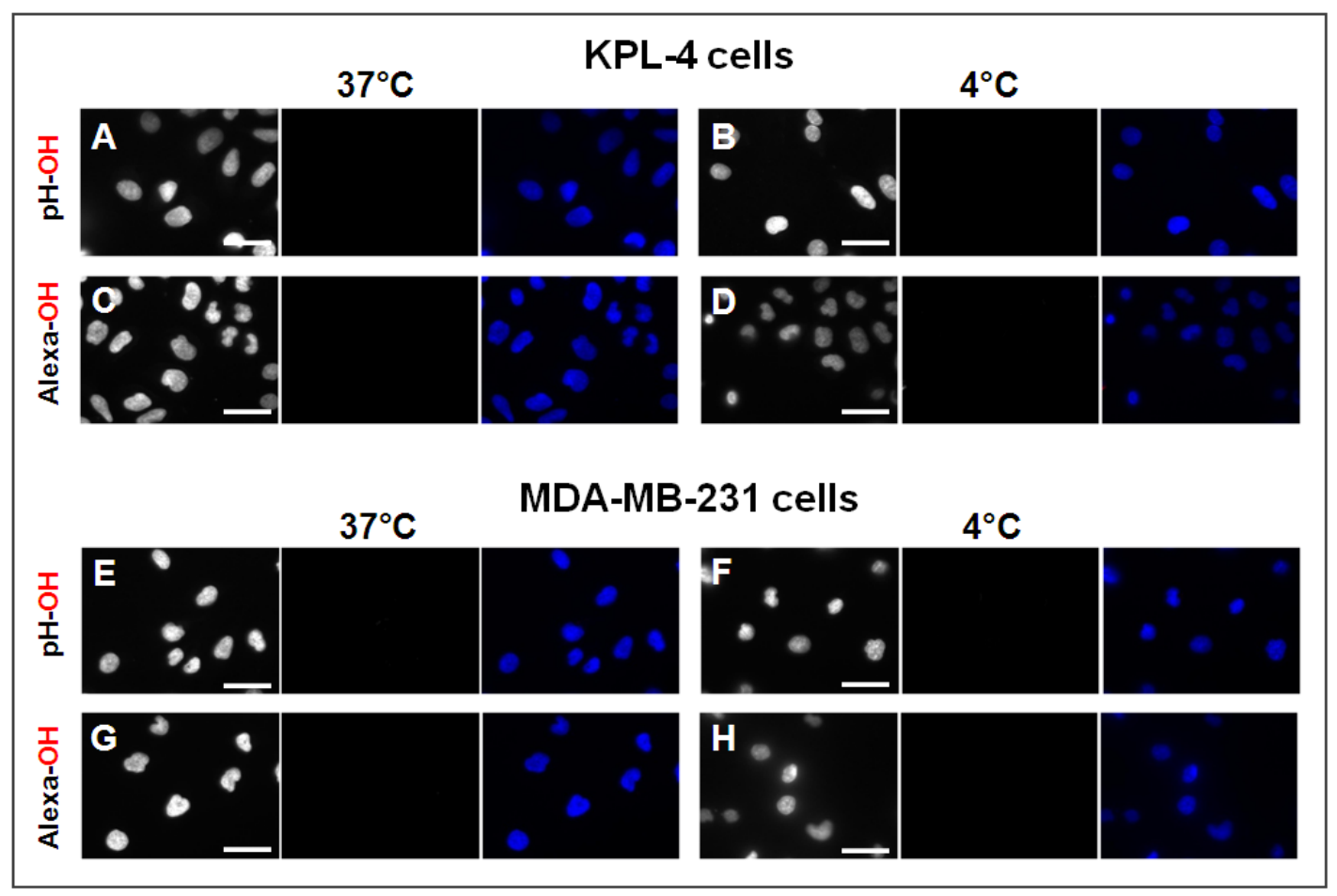

Figure 18. In vitro fluorescence microscopy reveals no unspecific binding of free fluorescence dyes

Cells grown on culture slides were examined under a fluorescence microscope at with an $\times 40$ objective. For this purpose cells were incubated for $8 \mathrm{~h}$ with $0.2 \mathrm{nmol}$ of $\mathrm{pH}-\mathrm{OH}$ or Alexa-OH. On the left, counter stain of cell nuclei with Hoechst 33342, in the middle, probe-derived signals, and on the right, merged images of the cell nuclei (blue) and the probe (red) are illustrated. For $\mathrm{pH}-\mathrm{OH}$ an exposure time of 1500 $\mathrm{ms}$ and for Alexa-OH an exposure time of $200 \mathrm{~ms}$ was used, according to the exposure times used for Herceptin-conjugates with low DP ratios (Figure 16). A.-H. No fluorescence can be detected on KPL-4 cells after $8 \mathrm{~h}$ of incubation with pH-OH at (A) $37^{\circ} \mathrm{C}$ and (B) $4{ }^{\circ} \mathrm{C}$ or with Alexa-OH at (C) $37{ }^{\circ} \mathrm{C}$ and (D) $4{ }^{\circ} \mathrm{C}$. Incubation of MDA-MB-231 cells with $\mathrm{pH}-\mathrm{OH}$ at $(\mathbf{E}) 37^{\circ} \mathrm{C}$ or $(\mathbf{F}) 4^{\circ} \mathrm{C}$ as well as with Alexa-OH at $(\mathbf{G})$ $37^{\circ} \mathrm{C}$ and $(\mathbf{H}) 4^{\circ} \mathrm{C}$ also does not result in the detection of fluorescence signals. Representative results of three independently performed experiments are shown. Bars represent $50 \mu \mathrm{m}$. 
The functionality of $\mathrm{pH}$-Her was confirmed by showing $\mathrm{pH}$-Her-derived fluorescence only after internalization in HER2-positive breast cancer cells presumably due to localization in acidic cell compartments. In contrast to that, fluorescence intensity of the always-on control probes, Alexa-Her was independent on $\mathrm{pH}$ and internalization.

\subsubsection{Improved detection sensitivity of tumors by the combined use of pH-sensitive probes and subtraction of autofluorescence}

$\mathrm{pH}$-probes were tested for their ability to improve tumor-detection sensitivity in tumor-bearing mice. A further aim of this study was to eliminate autofluorescence signals deriving from the mouse itself, hampering the detection sensitivity of specific, probe-derived signals. Consequently, imaging data were processed by the use of two different techniques for background suppression, subtraction of autofluorescence and LT-gated imaging.

For this purpose, KPL-4 and MDA-MB-231 breast tumor-bearing nude mice were imaged before (prescan) and at different times up to $72 \mathrm{~h}$ after $i . v$. injection of $25 \mu \mathrm{g}$ IgG of the respective probe in the Optix MX2.

\subsubsection{Background suppression via subtraction of autofluorescence in combination with pH-Her improves tumor- detection sensitivity}

In order to eliminate autofluorescence of mice and thus to only illustrate signals deriving from the injected probes, subtraction of autofluorescence was performed. For this purpose, the average fluorescence intensity over the tumor area of the untreated mouse (prescan) was subtracted from all scans of the same mouse. In Figure 19, representative fluorescence intensity maps of KPL-4 tumor-bearing mice before and 1 to $72 \mathrm{~h}$ after injection of the probes are demonstrated. After i.v. application of $\mathrm{pH}$-Her DP 1.6 (Figure 19A; $\mathrm{n}=3$ ) fluorescence in the tumor (white circle, shown at $24 \mathrm{~h}$ ) can be clearly detected. The background fluorescence (white circle, shown at $24 \mathrm{~h}$ ) is low and comparable to the fluorescence of the prescan, with exception of some signals deriving from the gastrointestinal (GI) tract of the mouse. In contrast, after injection of Alexa-Her DP 1.3 (Figure 19B; $n=3$ ), fluorescence in the tumor (white circle, shown at $24 \mathrm{~h}$ ) is also clearly visible but background signals (white circle, shown at $24 \mathrm{~h}$ ) are high compared to signals of the prescan. Note also, that fluorescence intensities after injection of Alexa-Her in mice (Figure 19B) are much higher compared to signals after injection of $\mathrm{pH}$-Her (Figure 19A) as indicated by the different scale bars in the figure. 


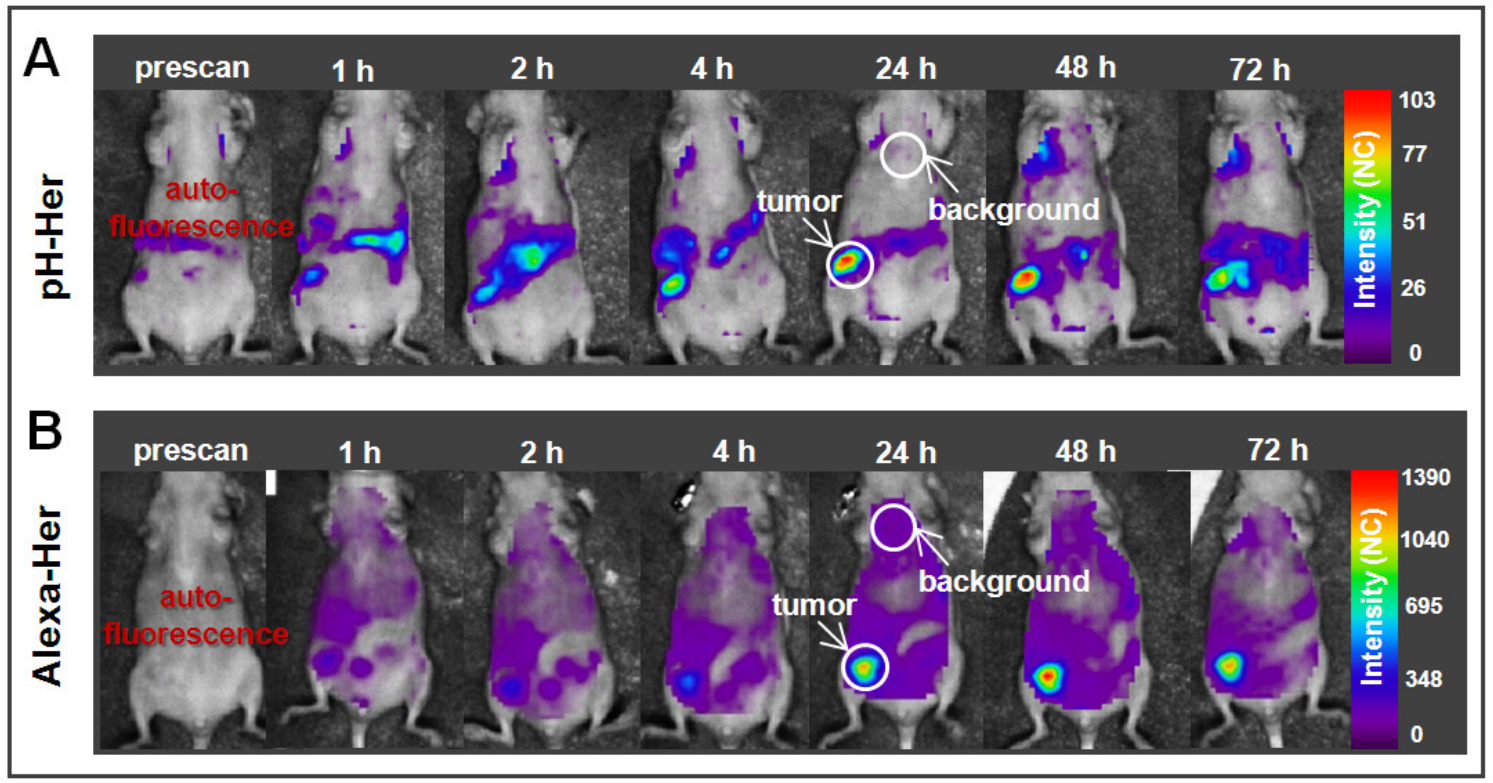

Figure 19. Intensity maps after subtraction of autofluorescence after probe application in vivo

KPL-4 breast tumor-bearing mice were imaged before and at different times up to $72 \mathrm{~h}$ after probe injection (25 $\mathrm{\mu g} \mathrm{IgG)} \mathrm{in} \mathrm{the} \mathrm{Optix} \mathrm{MX2} \mathrm{in} \mathrm{a} \mathrm{ventral} \mathrm{position.} \mathrm{Representative} \mathrm{examples}(\mathrm{n}=3)$ of fluorescence intensity images after subtraction of the mean autofluorescence, calculated over the tumor area of the respective untreated mouse (prescan) are shown. A. Images of a mouse receiving pH-Her DP 1.6 are illustrated. Probe-derived signals are mainly detected in the tumor. B. Images of a mouse receiving Alexa-Her DP 1.3 are illustrated. Probe-derived signals are detected in the tumor and in the background. Background- and tumor area are indicated with white circles. Note, that fluorescence intensities in B. are much higher than in A., as indicated by the different scale bars.

In summary, use of the $\mathrm{pH}$-sensitive probe, $\mathrm{pH}-\mathrm{Her}$ at a low DP ratio in combination with subtraction of autofluorescence resulted in elimination of major parts of background fluorescence in scans of tumor-bearing mice. In contrast, the intensity of the background strongly increased after injection of the always-on probe, Alexa-Her and could not be eliminated by subtraction of autofluorescence.

Contrast to noise ratios (CNRs) were calculated to quantify signal-detection sensitivity of the tumor in in vivo scans after background subtraction. The CNR provides information about the level of signal difference in the tumor and background and the affection of the signal by the background noise (see 2.2.8.4). CNRs were calculated in scans of KPL-4 tumor-bearing mice after treatment with Herceptinconjugates. As controls, CNRs were also determined in scans of MDA-MB-231 tumorbearing mice injected with Herceptin-conjugates and of KPL-4 tumor-bearing mice receiving the free fluorophores. As shown in Figure 20, i.v. injection of pH-Her DP 1.6 (Figure 20A) in HER2-positive KPL-4 tumor-bearing mice $(n=5)$ resulted in an continuous increase of CNRs within $24 \mathrm{~h}$ to a ratio of 171 that stays comparable high 
up to 72 h. In contrast, Alexa-Her DP 1.3 (Figure 20B; $n=5$ ) shows an increase in CNRs in these mice over time but only to a maximum CNR of 82 after $72 \mathrm{~h}$. The average CNR over all times in KPL-4 mice treated with pH-Her DP 1.6 was about 2.5fold higher than for mice treated with Alexa-Her.

Control experiments performed in HER2-negative MDA-MB-231 tumor-bearing mice injected with pH-Her DP 1.6 (Figure 20A; $\mathrm{n}=3$ ) or Alexa-Her DP 1.3 (Figure 20B; $\mathrm{n}$ = 3) revealed much lower CNRs, with an average of about 18 , that is 9-times lower than in KPL-4 tumor-bearing mice treated with $\mathrm{pH}-\mathrm{Her}$. In contrast to that, for the same times, the average CNR after application of Alexa-Her DP 1.3 is only 4-times higher in KPL-4 tumor-bearing mice compared to MDA-MB-231 tumor-bearing mice. This illustrates that the differences of signals deriving from HER2-positive- and HER2negative tumors can be enhanced by the use of the $\mathrm{pH}$-sensitive probe.
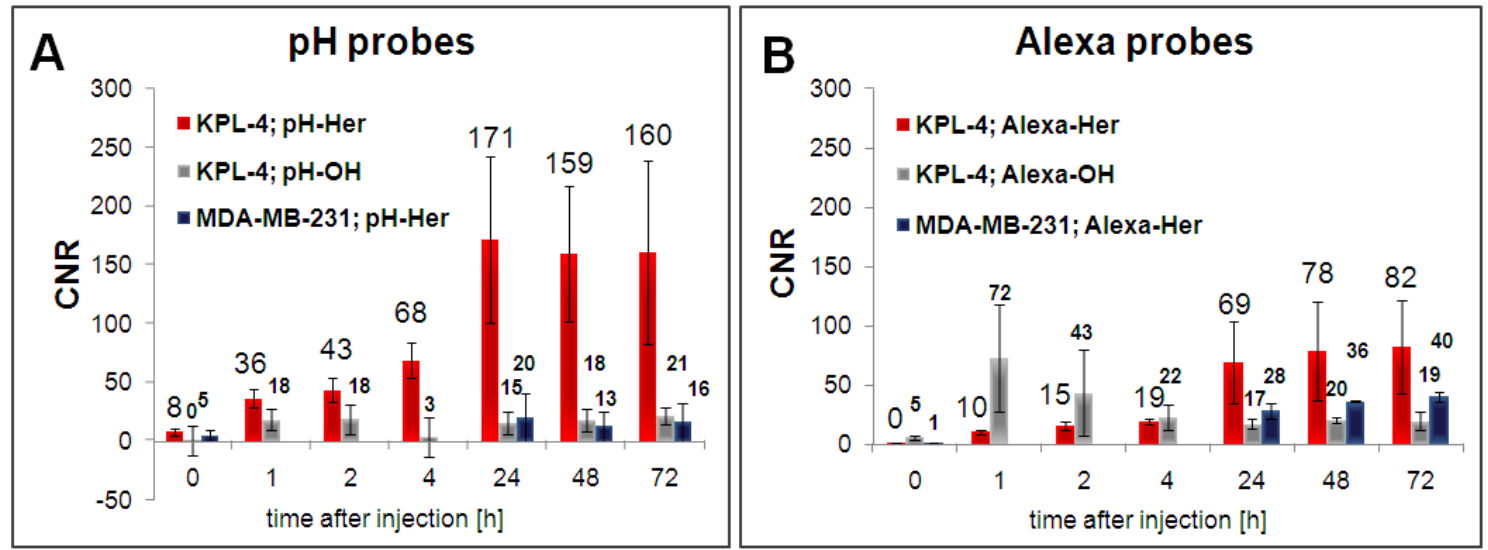

Figure 20. Improved tumor-detection sensitivity in vivo by use of a $\mathrm{pH}$-sensitive tumor-specific probe at a low DP ratio

A,B. CNRs calculated from in vivo intensity scans after subtraction of autofluorescence are shown. For calculation, the average intensity of the background was measured in the area over the lung. CNRs were calculated in scans of KPL-4 tumor-bearing mice before $(0 h)$ and 1, 2, $4(n=3), 24,28$, and $72 \mathrm{~h}(\mathrm{n}=5)$ after injection of (A) pH-Her DP 1.6 or (B) Alexa-Her DP 1.3. As controls, CNRs were also calculated from scans of KPL-4 tumor-bearing mice 0 to $72 \mathrm{~h}$ after injection of the free fluorescence dyes $(0.8 \mathrm{nmol} ; \mathrm{n}=$ 3), (A) $\mathrm{pH}-\mathrm{OH}$ and (B) Alexa-OH as well as from scans of MDA-MB-231 tumor-bearing mice 0, 24, 48, and $72 \mathrm{~h}(\mathrm{n}=3)$ after injection of $(\mathbf{A}) \mathrm{pH}-$ Her DP 1.6 or (B) Alexa-Her DP 1.3. Standard deviations are indicated as black bars.

Further control experiments in KPL-4 tumor-bearing mice revealed that after injection of the free dyes, CNRs also are low after 24 to $72 \mathrm{~h}$ with a maximum ratio for $\mathrm{pH}-\mathrm{OH}$ of 21 (Figure 20A; $\mathrm{n}=3$ ) and of 20 for Alexa-OH (Figure 20B; $n=3$ ). These findings further suggest that signals after application of both Herceptinconjugates, $\mathrm{pH}-\mathrm{Her}$ and Alexa-Her to mice with KPL-4 tumors are deriving from specific interaction of the antibody with tumor cells, as here much higher CNRs were 
calculated. Moreover, the fact that CNRs calculated for mice with MDA-MB-231 tumors after injection of $\mathrm{pH}-\mathrm{Her}$ and Alexa-Her are comparable to the CNRs in mice that received free fluorophores, indicates that tumor signals in MDA-MB-231 tumorbearing mice are deriving from unspecific accumulation of the Herceptin probe.

Surprisingly, for Alexa-OH administered to KPL-4 tumor-bearing mice, relatively high CNRs, of 72 were calculated $1 \mathrm{~h}$ after injection (Figure 20B). Furthermore, for both free dyes, $\mathrm{pH}-\mathrm{OH}$ and Alexa-OH high intensity signals were observed over the bladder at early ( 1 and $4 \mathrm{~h}$ ) times after probe injection, very likely deriving from a rapid excretion of the low molecular weight ( $1000 \mathrm{Da})$ molecules via the urinary system.

In addition, to analyze in more detail whether the fluorescence probes unspecifically accumulate in any other organ than the tumor, KPL-4 and MDA-MB-231 tumorbearing mice were sacrificed $72 \mathrm{~h}$ after injection of $\mathrm{pH}-\mathrm{Her}$, Alexa-Her, or the free fluorescence dyes and organs were scanned ex vivo. When compared to organs of untreated mice ( $n=4$ ), no remarkable fluorescence signals could be detected in liver, kidneys, lung, heart, spleen, stomach, and gut (data not shown).

Taken together, subtraction of autofluorescence in vivo in combination with application of $\mathrm{pH}-\mathrm{Her}$ at a low DP ratio led to an efficient elimination of background fluorescence in breast tumor-bearing mice and clearly improved tumor-detection sensitivity compared to injection of Alexa-Her.

\subsubsection{Background suppression via LT-gating does not improve tumor-detection sensitivity in vivo}

LT imaging in vivo not only illustrates specific signals deriving from the applied probe but can depict signals occurring from autofluorescence of the tissue and from unspecific fluorescence, e.g. from the food in the GI tract. First, in order to characterize this fluorescence from different origin, the LTs were analyzed in distinct areas of whole body scans of tumor-bearing mice prior to probe application. The representative histogram in Figure 21 , on the left side shows the frequency of occurrence of different LTs measured in an untreated KPL-4 tumor-bearing mouse ( $\mathrm{n}$ = 10). The most frequently detected LTs are shorter than 1 ns or longer than 2 ns. The corresponding color-coded LT map (Figure 21, right side) illustrates that the short LTs are deriving from autofluorescence in the background, which has a mean LT of $1.0 \mathrm{~ns}$ ( $n=10$ ) whereas the longer LTs correspond likely to the food in the GI tract, with a mean LT of $2.0 \mathrm{~ns}(n=10)$. The LT of the autofluorescence in the tumor with a mean of $1.6 \mathrm{~ns}(\mathrm{n}=10)$ is higher than the $L T$ in the background. 


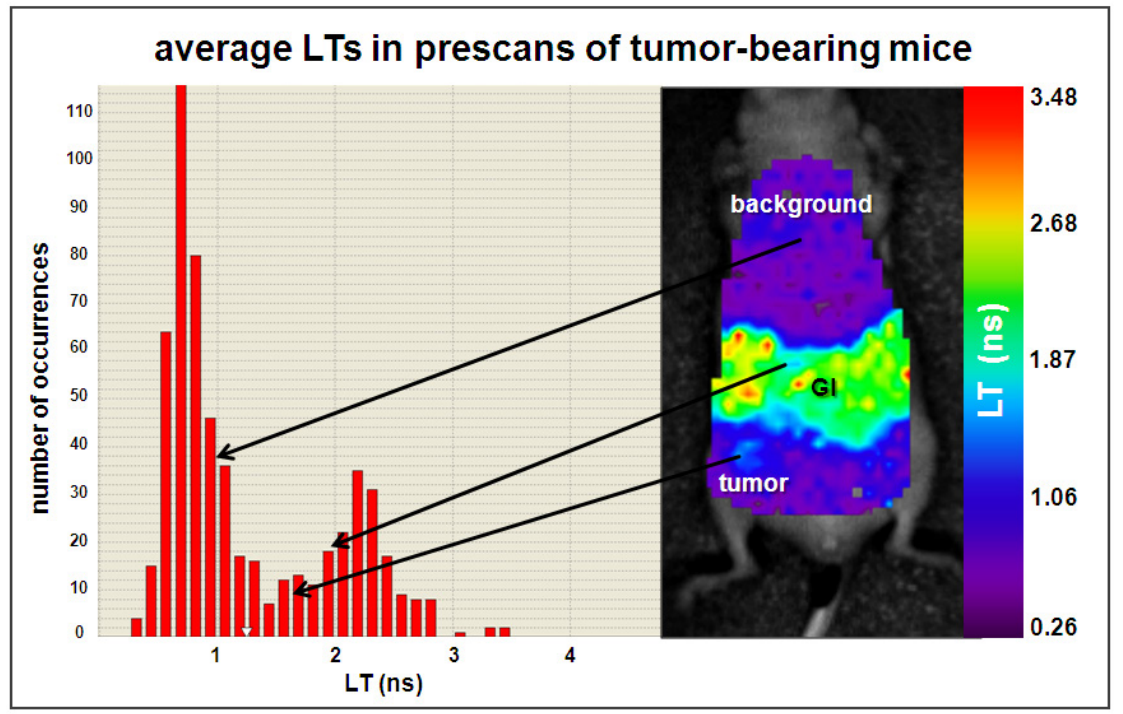

Figure 21. LTs of fluorescence signals in certain areas of untreated tumorbearing mice

On the left side, a representative histogram shows the frequency of occurrence of different LTs measured in an untreated KPL-4 tumorbearing mouse $(n=10)$ in the Optix $M \times 2$. The most frequently

measured LTs are shorter than 1 ns or longer than 2 ns. On the right side, the corresponding color-coded LT map of an untreated mouse is shown. The autofluorescence in the background has a mean LT of $1.0 \mathrm{~ns}$ (arrow; $n=10$ ). In contrary, the GI tract (GI) has a mean LT of 2.0 ns (arrow; $n=10$ ). The mean LT of the autofluorescence in the tumor is $1.6 \mathrm{~ns}$ (arrow).

With the aim to only illustrate fluorescence signals in vivo, that are deriving from the applied probe, intensity maps of mice were gated to the predicted LT of the applied probe $\pm 0.2 \mathrm{~ns}$ in order to decouple them from unspecific background fluorescence. The predicted LT was calculated as the mean from the LTS of the respective probe determined in vitro under different conditions and after s.c. injection in mice (see section 3.2.2, Table 10). In Figure 22A, representative fluorescence intensity maps of a mouse bearing a KPL-4 tumor in the right abdominal mammary gland are illustrated at different times after receiving pH-Her DP $1.6(n=5)$. Only signals with LTs of $1.3 \mathrm{~ns} \pm 0.2 \mathrm{~ns}$ are shown, according to the predicted LT for $\mathrm{pH}-\mathrm{Her}$ (Table 10). When comparing these LT-gated images to the ones after subtraction of background fluorescence of the same mice, in Figure 19A, some fluorescence is still visible in the background (white circle, shown at $24 \mathrm{~h}$ ) whereas unspecific fluorescence from the GI tract of mice could be completely eliminated. Unexpectedly, the fluorescence signals of high intensity from $\mathrm{pH}-\mathrm{Her}$ in the tumor (white circle, shown at $24 \mathrm{~h}$ ) are also eliminated at most times (e.g. at 48 and $72 \mathrm{~h}$ ). Figure 22B, shows representative fluorescence intensity maps of a mouse with a KPL-4 tumor receiving Alexa-Her DP $1.3(n=5)$. Only signals with LTs of $1.5 \mathrm{~ns} \pm 0.2$ ns are shown, according to the predicted LT for Alexa-Her (Table 10). In contrary to LTgating after application of $\mathrm{pH}-\mathrm{Her}$, the major part of the background fluorescence is still visible (white circle, shown at $24 \mathrm{~h}$ ) whereas fluorescence signals from Alexa-Her in the tumor (white circle, shown at $24 \mathrm{~h}$ ) are also partly eliminated at some time points (e.g. at 4 and $24 \mathrm{~h}$; see also Figure $19 \mathrm{~B}$ for comparison). 


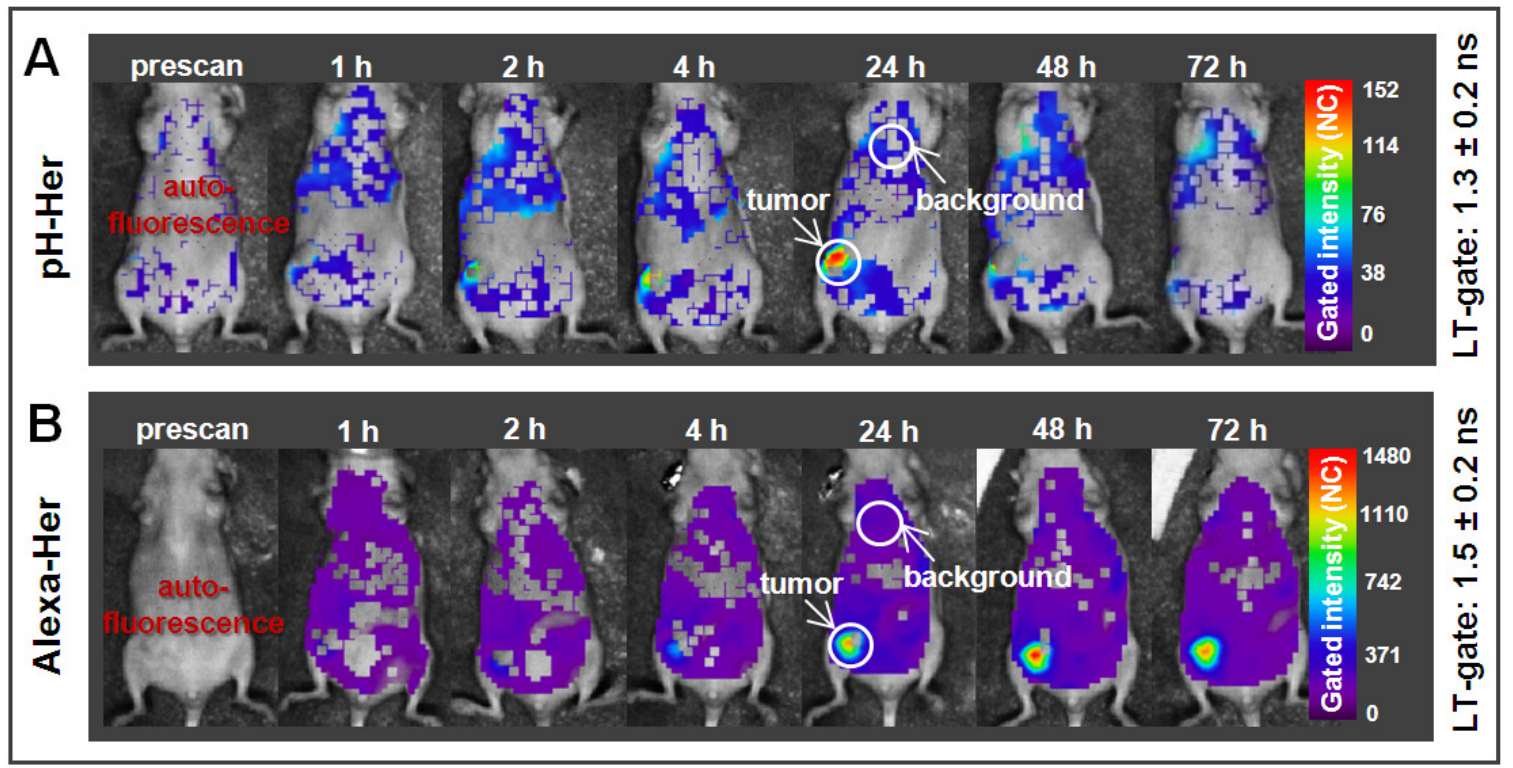

\section{Figure 22. LT-gated intensity maps after probe application in vivo}

$\mathrm{KPL}-4$ breast-tumor bearing mice were imaged before and from 1 to $72 \mathrm{~h}$ after probe injection (25 $\mu \mathrm{g} \mathrm{IgG)}$ in the Optix MX2 in a ventral position. Representative examples of LT-gated in vivo intensity maps are shown. The LT-gates were set according to the predicted LT of the respective probe \pm 0.2 ns. A. A representative mouse receiving $\mathrm{pH}-\mathrm{Her}$ DP 1.6 is illustrated $(n=3)$. After LT-gating (LT: $1.3 \pm 0.2 \mathrm{~ns})$ remaining background fluorescence and partial elimination of the tumor signals are observed at most time points (e.g. after $48 \mathrm{~h})$. B. A representative mouse receiving Alexa-Her DP 1.3 is illustrated $(n=3)$. After LT-gating (LT $1.5 \pm 0.2 \mathrm{~ns}$ ) a high amount of background fluorescence remains and part of the tumor signal is eliminated at some time points (e.g. after 4 and $48 \mathrm{~h}$ ). Background- and tumor area are indicated with white circles at $24 \mathrm{~h}$ after probe injection. Note, that fluorescence intensities in $\mathrm{B}$. are much higher than in A., as indicated by the different scale bars.

Here, LT-gating does not lead to an improved tumor-detection sensitivity in vivo as probe-derived signals in the tumor are eliminated, especially those of $\mathrm{pH}$-Her. In addition, LT-gating does not reduce fluorescence in the background after injection of the always-on fluorescent probe, Alexa-Her.

The average LTs in the tumor at each time point were analyzed more precisely in order to evaluate why part of tumor signals was eliminated after LT-gating, although high intensities in the tumor were very likely deriving from the applied fluorescence probes. Figure 23 shows that the average LTs in the tumor area of KPL-4 tumorbearing mice (M1 to M5), each treated with either pH-Her DP 1.6 (Figure 23A) or Alexa-Her DP 1.3 (Figure 23B) vary over time and in the different mice. Moreover, after application of $\mathrm{pH}-\mathrm{Her}$ (Figure 23A) tumor LTs are clearly longer than the predicted LTs (black dashed line; Table 10) and are also partly outside the LT-range used for gating $(1.3 \pm 0.2 \mathrm{~ns}$, grey area) of $\mathrm{pH}-\mathrm{Her}$ signals. The mean LT in the KPL4 tumor area of all mice treated with $\mathrm{pH}-\mathrm{Her}$ and at all times is $1.49 \pm 0.06 \mathrm{~ns}(\mathrm{n}=$ 
24). Ex vivo LT analyses of the excised tumors $72 \mathrm{~h}$ after injection of $\mathrm{pH}-\mathrm{Her}$ reveal an even higher mean LT of $1.57 \pm 0.13 \mathrm{~ns}$ ( $\mathrm{n}=5$; data not shown). In contrary, the average LTs after injection of Alexa-Her (Figure 23B) are close to the predicted LT (black dashed line; Table 10) and are within the LT-gate (1.5 $\pm 0.2 \mathrm{~ns}$, grey area) determined for Alexa-Her. Here, the mean LT in the tumor area over time of all mice is $1.48 \pm 0.04 \mathrm{~ns}(\mathrm{n}=24)$. Ex vivo $\mathrm{LT}$ analyses of the tumors $72 \mathrm{~h}$ after injection of Alexa-Her also reveal a higher mean LT of $1.65 \pm 0.05 \mathrm{~ns}(\mathrm{n}=5$; data not shown). This demonstrates that for both Herceptin probes the LT does further increase when the tumor is measured ex vivo where its fluorescence signal is not overlayed with other signals deriving from mouse tissue.
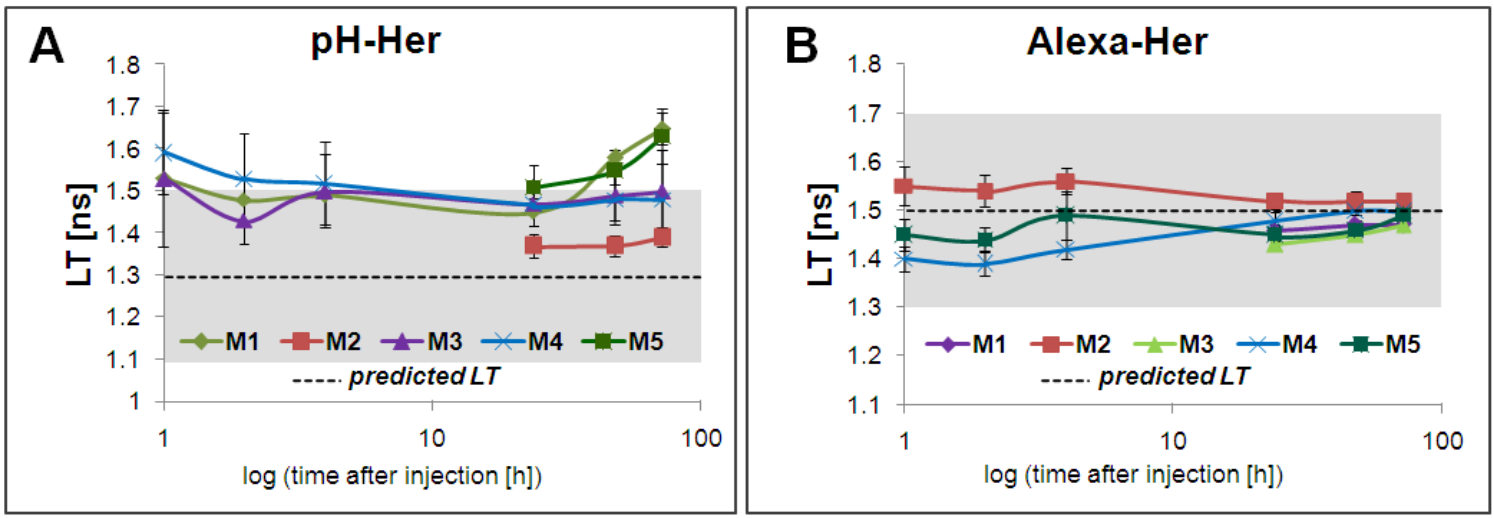

Figure 23. Variations of LTs in the tumor area after probe application

In vivo average LTs in KPL-4 tumors of mice (M1 - M5) are shown. Tumor LTs were analyzed 1, 2, 4 ( $n=$ 3), 24, 48, and $72 \mathrm{~h}(\mathrm{n}=5)$ after i.v. injection of fluorescence probes. A,B. The predicted LT of the probe (determined in in vitro measurements and after s.c. injection) is indicated as a black dashed line and the LT-ranges used for LT-gating of in vivo intensity images are shown in grey. LTs after application of $(\mathbf{A}) \mathrm{pH}-$ Her DP 1.6 and (B) Alexa-Her DP 1.3 are shown. LTs vary over time as well as between mice. Note, that tumor LTs after application of $(\mathbf{A}) \mathrm{pH}-\mathrm{Her}$ are partly longer than the predicted LT. Standard deviations are indicated as bars.

In summary, LT-analyses of tumor signals over time in vivo and ex vivo show that the predicted $\mathrm{LT}$ for $\mathrm{pH}-\mathrm{Her}$ differs from the $\mathrm{LT}$ of the probe in the tumor. Therefore, a clear prediction of the in vivo LT of the probe in the KPL-4 tumor environment and thus an identification of probe-derived signals via LT-gating is not possible.

In order to examine the reliability of the LT calculations in the tumor, the LT goodness of fit was determined. This parameter determines the quality of the mean LTs calculated over the tumor area of the respective mice. A LT-calculation with a high accuracy is considered to have a LT goodness of fit between 0.8 and 1.2 (see also section 2.2.8.4 in Material and Methods). As can be seen in Figure 24, the LT goodness of fit does not significantly increase or decrease from 1 to $72 \mathrm{~h}$ after probe 
application or between the mice. For tumor LTs after injection of pH-Her (Figure 24A) as well as of Alexa-Her (Figure 24B) the mean goodness of fit is $0.8(n=24)$ indicating that the LT calculations have a high accuracy.
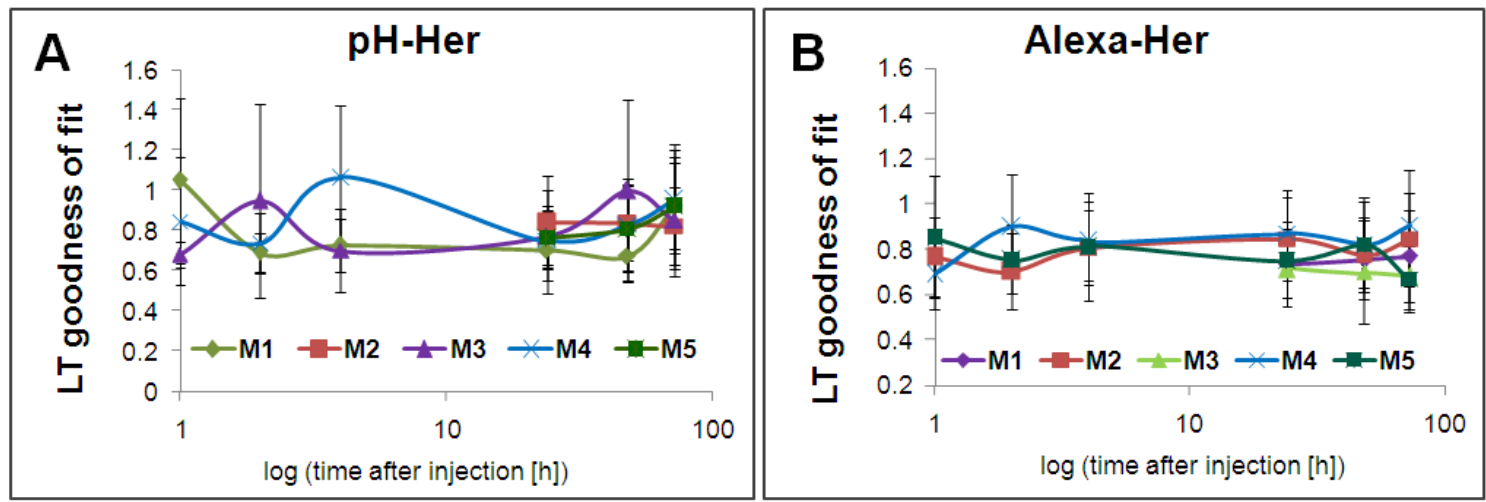

Figure 24. Analysis of reliability of in vivo LT calculations

The LT goodness of fit of the in vivo LT calculation in the KPL-4 tumor area in mice (M1 - M5) at different times after injection of (A) pH-Her DP 1.6 and (B) Alexa-Her DP 1.3 shows slight variations over time as well as between the mice. The mean LT goodness of fit for the LT calculations of both probes is 0.8 . Standard deviations are indicated as bars.

These results demonstrate that the in vivo LT calculations of the tumor area are reliable, even at early $(1 \mathrm{~h})$ and late $(72 \mathrm{~h})$ times after injection of the respective probe when the concentration of the fluorescent probe in the tumor might be low.

In summary, tumor-detection sensitivity in vivo was not enhanced after injection of Herceptin-conjugates in combination with LT-gating. As prediction of the pH-Her LT was not precise and background signals deriving from unbound Alexa-Her could not be efficiently eliminated, tumor-derived fluorescence was not effectively separated from background fluorescence.

\subsection{5. pH-Her conjugates at high DP ratios are less suitable for in vivo imaging}

Fluorescence probes with high DP ratios, pH-Her DP 5.0 and Alexa-Her DP 3.4 were injected in KPL-4 tumor-bearing mice $(n=3)$ to evaluate their tumor-detection sensitivity in vivo in comparison to fluorescence probes with low DP ratios. For this purpose, CNRs were calculated from in vivo fluorescence intensity scans of KPL-4 tumor-bearing mice receiving probes at high DP-ratios after background subtraction. As depicted in Figure 25, after injection of pH-Her DP 5.0, CNRs over time stay very low with a maximum ratio of 7 after $72 \mathrm{~h}$ (Figure 25A). In addition, during in vivo scans we could detect only weak tumor signals but interestingly strong fluorescence in the bladder of the mice especially at early times ( 1 to $4 \mathrm{~h}$ ) after application of $\mathrm{pH}$ - 
Her DP 5.0 ( $n=3$; data not shown). This was also observed after injection of $\mathrm{pH}-\mathrm{Her}$ DP 6.4 (data not shown; $n=2$ ) and after application of the free fluorescence dyes, $\mathrm{pH}-\mathrm{OH}$ and Alexa-OH (see 3.2.4.1) in tumor-bearing mice.

CNRs after injection of Alexa-Her DP 3.4 in KPL-4 tumor-bearing mice increased over time to a maximum ratio of 47 . However, the average CNR at all times was $\sim 1.7$ fold lower compared to the average CNRs in the same mice model after injection of the Alexa-Her conjugate with a low DP ratio (see 3.2.4.1, Figure 20B).

Human IgG has a hydrodynamic diameter (HD) of $10 \mathrm{~nm}$ and usually gets excreted via the liver ${ }^{75}$. The observation of a rapid excretion of the $\mathrm{pH}-\mathrm{Her}$ probe at high DP ratio via the kidneys and bladder which is usually the case for smaller molecules of 5 to $6 \mathrm{~nm}$ in $\mathrm{HD}^{75}$ pointed already to a structural degradation of the Herceptinconjugates into smaller fragments. As CNRs were low for Herceptin-conjugates with high DP ratios, when compared to CNRs of conjugates with low DP ratios, control studies on MDA-MB-231 tumor-bearing mice or with free fluorophores were not performed.
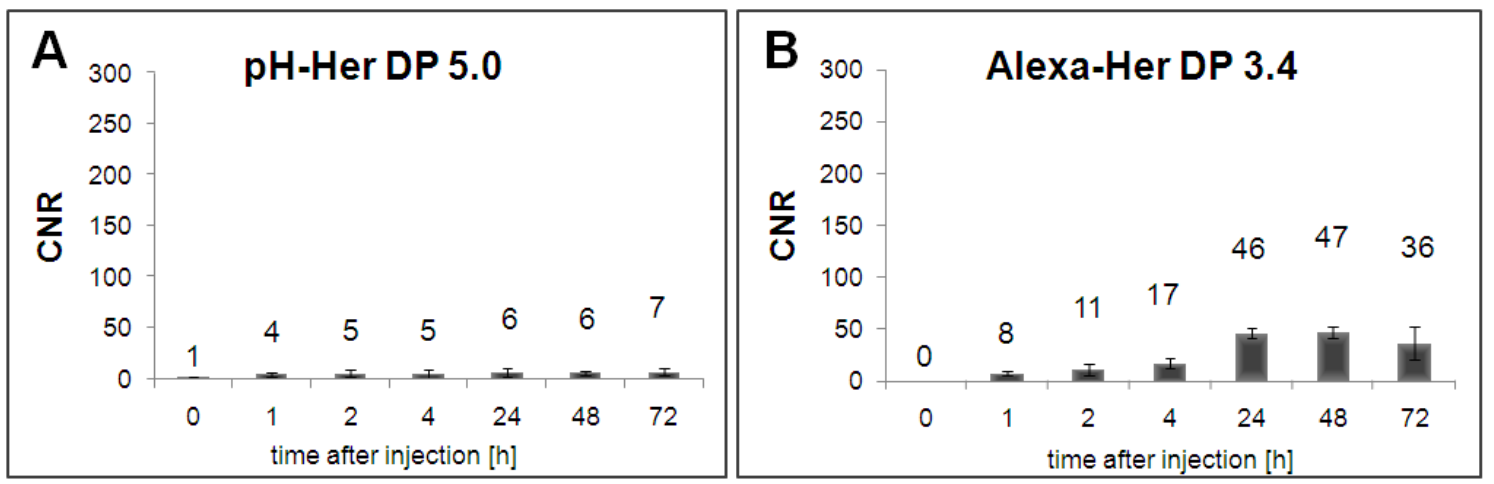

Figure 25. Fluorescence probes with high DP ratios reveal no improvement in tumor-detection sensitivity

CNRs were calculated from fluorescence intensity scans of KPL-4 tumor-bearing mice after background subtraction before $(0 \mathrm{~h})$ and 1 to $72 \mathrm{~h}(\mathrm{n}=3)$ after injection of probes ( $25 \mu \mathrm{g} \mathrm{IgG})$ at high DP ratios. CNRs after application of (A) pH-Her DP 5.0 and (B) Alexa-Her DP 3.4 are shown. For CNR-calculation the average intensity of the background was measured in the area of the lung. Standard deviations are indicated as black bars.

Taken together, the application of fluorescent probes with high DP ratios unexpectedly did not lead to an improved sensitivity of tumor detection in vivo, when compared to probes with low DP ratios. 
In the following it was assessed, whether $\mathrm{pH}-\mathrm{Her}$ at high DP ratios might have been decomposed to nonfunctional fragments at the time of in vivo application in tumorbaring mice, in comparison to their application in vitro on cells (see section 3.2.3, Figure 16 and 17).

For this purpose, first fluorescence probes were separated according to their size by SDS-PAGE under non-reducing conditions as shown in Figure 26. As a control, unlabeled Herceptin in its reduced and unreduced form was used. In order to separate the heavy ( $\sim 50 \mathrm{kDa}$ ) and the light chain ( $25 \mathrm{kDa})$ of the antibody, Herceptin was incubated with reducing agent and heated at $70{ }^{\circ} \mathrm{C}$ for 10 min (line A). Herceptin in its unreduced form has a size of $\sim 146 \mathrm{kDa}$ (line B). Compared to the size of this antibody, the CypHer5E- and Alexa Fluor 647 fluorophores are small (750 to $1200 \mathrm{Da}$ ) so that the size of the fluorescence conjugates does not significantly differ from that of the free IgG. Alexa-Her DP 1.3 (line C) and DP 3.4 (line D) as well as pH-Her DP 1.6 (line E) reveal a comparable strong band with the size of unreduced Herceptin. pH-Her DP 1.6 additionally shows two bands with the size of $250 \mathrm{kDa}$ and larger which are likely to be deriving from agglomerates of the conjugates. In contrast, pH-Her DP 5.0 (line F) and DP 6.4 (line G), both show no bands at the size of unreduced Herceptin and only some very weak bands between 25 and $45 \mathrm{kDa}$, indicating that $\mathrm{pH}-\mathrm{Her}$ at high $\mathrm{DP}$ ratios has been decomposed into smaller fragments.

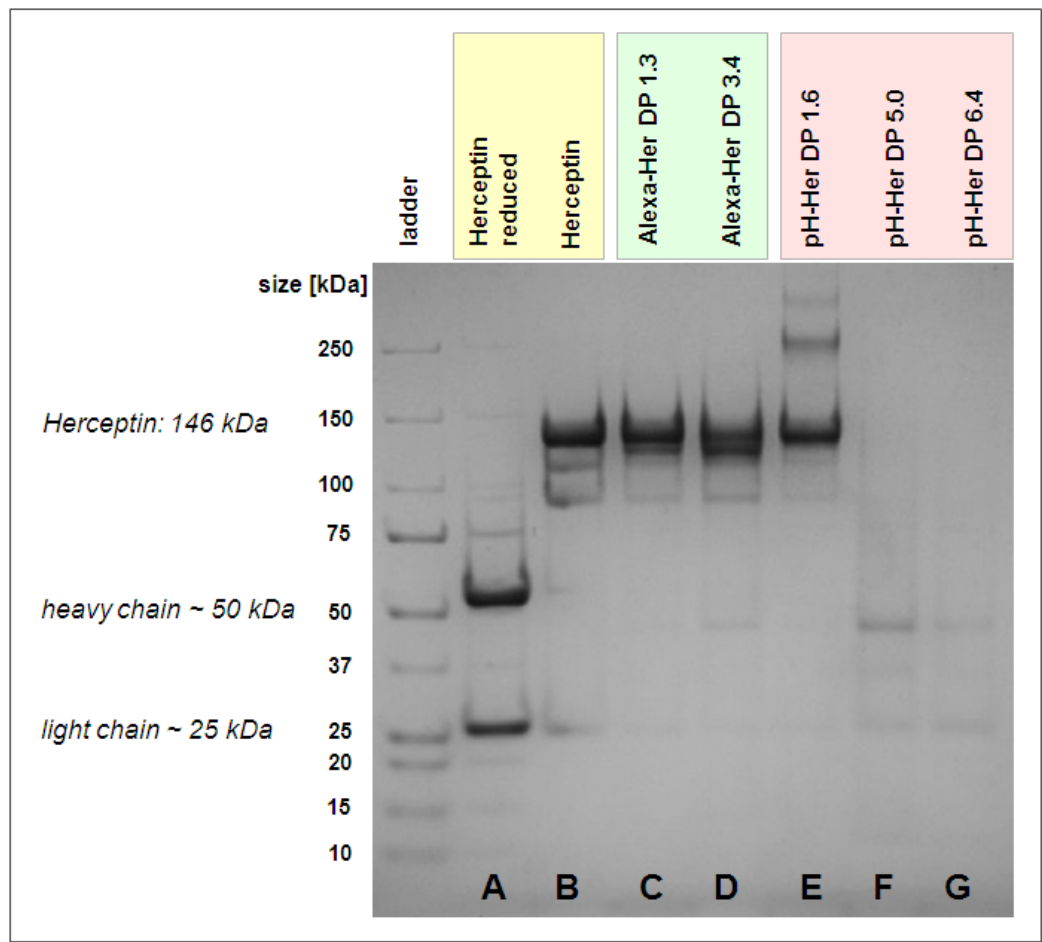

Figure 26. Detection of conjugates in a nonreducing protein gel

Herceptin-dye-conjugates (5 $\mu g \quad I g G)$ were separated according to their size via SDS-PAGE under nondenaturating conditions and stained with Coomassie ( $\mathrm{n}=$ 1). (A) As a control, unlabeled Herceptin was treated with reducing agent and heated to $70{ }^{\circ} \mathrm{C}$ for $10 \mathrm{~min}$. Heavy and light chains of the antibody can be seen. (B) Unlabeled Herceptin in its non-reduced form. (C) Alexa-Her DP 1.3 and (D) DP 3.4 as well as (E) pH-Her DP 1.6 have a size comparable to the unlabeled Herceptin. (F) pH-Her DP 5.0 and (G) DP 6.4 show no bands at the size of Herceptin. 
To verify whether the Herceptin-conjugates were destructed into smaller fragments, the protein content of the probes was determined in a BCA assay ( $n=2$; data not shown) revealing that $\mathrm{pH}-\mathrm{Her} \mathrm{DP} 5.0$ (line $\mathrm{F}$ ) and $\mathrm{pH}$-Her DP 6.4 (line $\mathrm{G}$ ) had an approximately 3 - and 5-times lower protein content, respectively when compared to the other probes (line B-E). Via this method, proteins decomposed to very small peptides containing three or more aminoacid residues are still detectable. Therefore, it can be assumed that the low functionality of the $\mathrm{pH}$-Her conjugates with high DP ratios in vivo might not solely been caused by a degradation of the antibody conjugates into smaller fragments, detected in vivo in the bladder but also by a partial precipitation, presumably caused by probe aggregation.

In general, fluorescence conjugates with low DP ratios tend to be more stable over time when compared to conjugates with high DP ratios. 


\subsection{Evaluation of Itrybe-loaded NPs as in vivo NIRF imaging agents}

Nanosized carrier systems are promising imaging tools as they exhibit several advantages, such as loading with multiple fluorophores resulting in highly intense and stable fluorescent labels. Furthermore, they enable surface conjugation with multiple target-specific ligands, which compared to the commonly used single antibody-fluorophore units may be especially favorable for in vivo imaging applications.

In this context, NPs with a diameter of $100 \mathrm{~nm}, 25 \mathrm{~nm}$, and $15 \mathrm{~nm}$, loaded with the NIR fluorophore Itrybe were evaluated for their capacity to be used for in vivo monitoring of tumors. These particles were either unmodified, bearing $\mathrm{NH}_{2}$ groups or PEG chains of 1.5 or $10 \mathrm{kDa}$, linked to the NPs via the $\mathrm{NH}_{2}$ groups. In addition, Herceptin-modified (Her) NPs were examined, at which the antibody was conjugated to the particle surface via the PEG chains (dye-loading and surface conjugation were performed by Thomas Behnke, BAM I.5, Berlin, see section 2.2.3.3).

\subsubsection{Characterization of Itrybe-loaded NPs}

Fluorescence excitation and emission spectra of different Itrybe-loaded NPs were recorded, to analyze whether the different sizes (100, 25, and $15 \mathrm{~nm}$ ) and surface modifications $\left(\mathrm{NH}_{2}, \mathrm{PEG}, \mathrm{Her}\right)$ influence their spectral behavior.

Figure 27A-C illustrates no remarkable spectral differences of NPs with the same size but different surface-modifications. However, the excitation and emission spectra of the $100 \mathrm{~nm}$ particles (blue line) are slightly right shifted compared to the spectra of the smaller sized NPs (25 nm and $15 \mathrm{~nm}$ ), as exemplarily shown for $\mathrm{NH}_{2}$-modified probes in Figure 27. All spectra appear noisy which might be caused by a decreased emission intensity due to scattering effects of the NPs itself. Scattering of the NPs can lead to a size-dependent decrease of excitation intensity and to interreflections of emitted photons and subsequently to a stronger reabsorption by the dye. This may cause the slight right shifts in the spectra of the $100 \mathrm{~nm}$ NPs (Figure 1D) compared to the smaller sized $15 \mathrm{~nm}$ and $25 \mathrm{~nm}$ NPs. With an increase in NP size, scattering and reabsorption effects increase possibly leading to some measurement artifacts. 


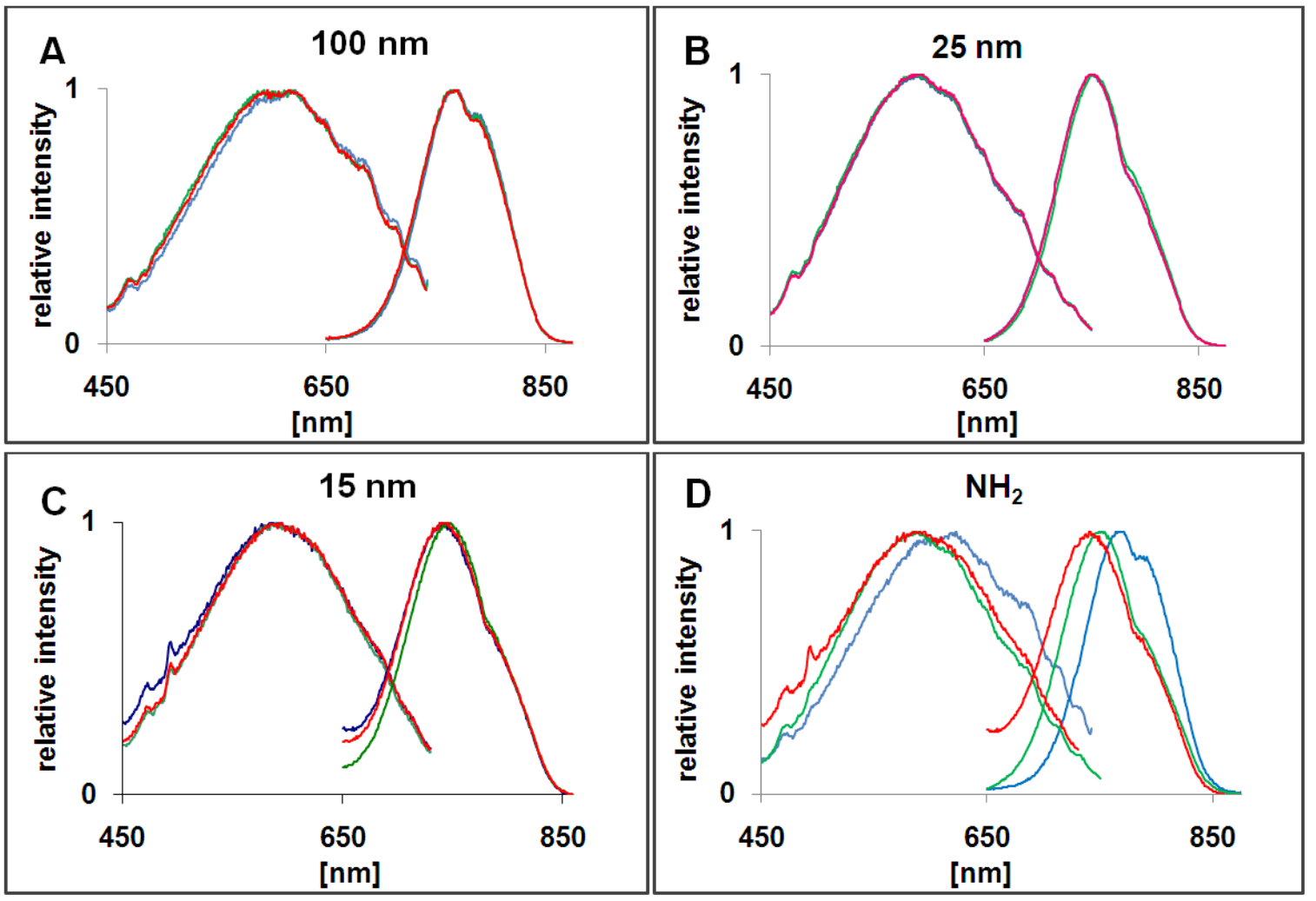

Figure 27. Fluorescence excitation and emission spectra of NPs

Representative normalized fluorescence spectra of Itrybe-loaded NPs of different sizes and surface modifications were measured in a spectrofluorometer. The excitation spectra range from 450 to $750 \mathrm{~nm}$. The emission spectra range from $\sim 650$ to $850 \mathrm{~nm}$. Fluorescence spectra of (A) $100 \mathrm{~nm}(\mathrm{n}=3),(B) 25 \mathrm{~nm}$ $(n=3)$, and $(\mathbf{C}) 15 \mathrm{~nm}(\mathrm{n}=2)$ NPs are illustrated. No remarkable spectral changes are observed for the different surface-modifications of NPs $\left(\mathrm{NH}_{2}, \mathrm{PEG}\right.$, and Her). D. Spectra of all three sizes of NPs with $\mathrm{NH}_{2}$ groups are illustrated. Note that, excitation and emission spectra of $100 \mathrm{~nm}$ particles (blue line) are slightly right shifted compared to the $25 \mathrm{~nm}$ and $15 \mathrm{~nm}$ NPs (green and red line).

Taken together, spectral differences of NPs of the same size but with various surface modifications are only small showing that the surface moiety has no relevant impact on the detection of Itrybe-derived fluorescence using the same excitation and emission settings. As fluorescence intensities from differently sized particles were not directly compared, the slight spectral variations of NPs of different sizes were not of relevance for the results of the study.

The encapsulation of the NIR fluorescence dye, Itrybe in NPs provides a cover of the dye towards the environment. Common factors influencing the fluorescence properties of fluorophores are, among others, proteins and ions ${ }^{2,20,108}$. Therefore, the influences of changes in the environment on Itrybe loaded in NPs were analyzed exemplarily for the different sizes of the $\mathrm{NH}_{2}$-modified particles ( $\mathrm{n}=2$, for each size and each condition). As depicted in Figure 28, a decrease in $\mathrm{pH}$ from 7.5 to 5.5 has 
no influence on fluorescence properties of the dye enclosed in NPs of all sizes; however a slight (1.3-fold) increase was observed for the 100 and $25 \mathrm{~nm}$ NPs when $5 \%$ BSA is added to the measurement solution (PBS), independent on $\mathrm{pH}$. In contrary, as can be seen in Figure 28C, Itrybe loaded in the smallest, $15 \mathrm{~nm}$ particles shows a stronger, 5 -fold increase in fluorescence in the presence of $5 \% \mathrm{BSA}$ at a neutral $\mathrm{pH}$. At an acidic $\mathrm{pH}$ of 5.5 the fluorescence is still 4 -fold higher compared to fluorescence measured in PBS.

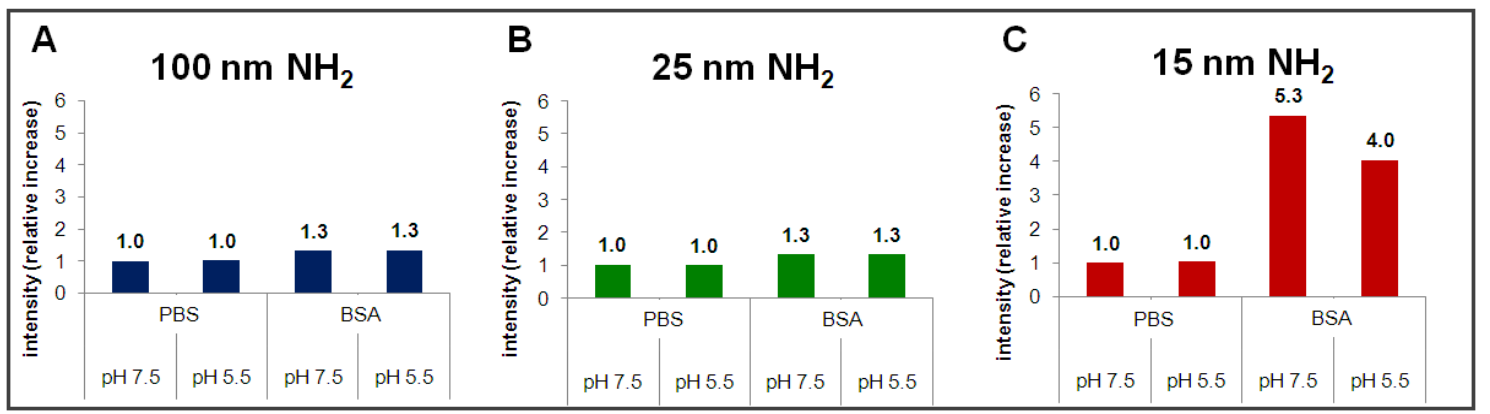

Figure 28. Sensitivity of NP-encapsulated Itrybe to changes in environment

The relative increase in fluorescence intensity of Itrybe-loaded $\mathrm{NH}_{2} \mathrm{NPs}$ of different sizes in dependence on environmental factors was analyzed in the Optix MX2. For this purpose, NPs were measured in PBS with a $\mathrm{pH}$ of 7.5 and 5.5 as well as in the presence of proteins ( $5 \%$ BSA). For relativization, the lowest mean intensity for each NP size was set to 1. A. 100 and B. $25 \mathrm{~nm}$ particles show no influence on fluorescence intensity with a change in $\mathrm{pH}$ but a slight (1.3-fold) increase in fluorescence in the presence of proteins. $\mathbf{C}$. In contrary, $15 \mathrm{~nm}$ NPs revealed a high (5.3-fold) increase in fluorescence in the presence of proteins at a $\mathrm{pH} 7.5$ that is slightly lower (4-fold) at a pH of 5.5. The mean of two measurements is illustrated for each probe and each condition.

In summary, the influence of proteins on Itrybe-derived fluorescence are relatively small in $100 \mathrm{~nm}$ and $25 \mathrm{~nm}$ particles but increase in the small, $15 \mathrm{~nm}$ NPs. Moreover, the $\mathrm{pH}$ shows no strong influence on fluorescence of Itrybe.

\subsubsection{Functionality of bioconjugated NPs}

Itrybe-loaded NPs were designed to specifically bind to tumor cells. For this purpose, Herceptin, which recognizes HER2, was bioconjugated to the 100, 25, and $15 \mathrm{~nm}$ NPs. Immunoassays were performed to evaluate whether 1.) bioconjugation of NPs was successful and 2.) Herceptin-conjugated NPs bind to the HER2 antigen, as well 3.) $\mathrm{NH}_{2}$-modified and PEGylated particles unspecifically bind to HER2 antigen-coated surfaces.

Figure 29 shows the ratio of fluorescence detected after incubation of HER2-coated and -uncoated wells with $15 \mathrm{~nm}$ particles. A ratio of 1.0 is determined for the control experiment in which coated and uncoated wells were incubated with TBS only. 
Particles with $\mathrm{NH}_{2}$ groups and PEG 10 kDa chains show no specific binding to antigencoated wells. To achieve a high conjugation efficiency of Herceptin to the relatively small $15 \mathrm{~nm}$ NPs und thus a specific binding to HER2, a 5-fold amount of Herceptin (Her $x 5 ; 2.4 \mathrm{nmol} / \mathrm{mg}$ PS; see section 2.2.3.3, Table 2) was used for coupling procedure. After Herceptin-conjugation (Her x5), NPs with 10 and 1.5 kDa PEG chains show a slightly increased binding ratio of 1.1 and 1.2 , respectively. To further increase specific binding of $15 \mathrm{~nm}$ NPs by Herceptin, in a next step the amount of PEG for coupling procedure was reduced to $1 / 10$ (PEG $1.5 \mathrm{kDa} 1 / 10 ; 50 \mathrm{nmol} / \mathrm{mg}$ PS; see section 2.2.3.3, Table 2 ) of the original amount. This was done, as a too high density of PEG chains might probably lead to steric hindrances during antibody conjugation. In addition, a "blockade" of the already conjugated antibodies on the particle surface can occur, thereby hampering interaction of the antibody with its target. However, when reducing the PEG amount for coupling procedure, after conjugation to Herceptin (Her $\times 5$ ) again no specific binding to the HER2 antigen is observed (ratio 1.0).

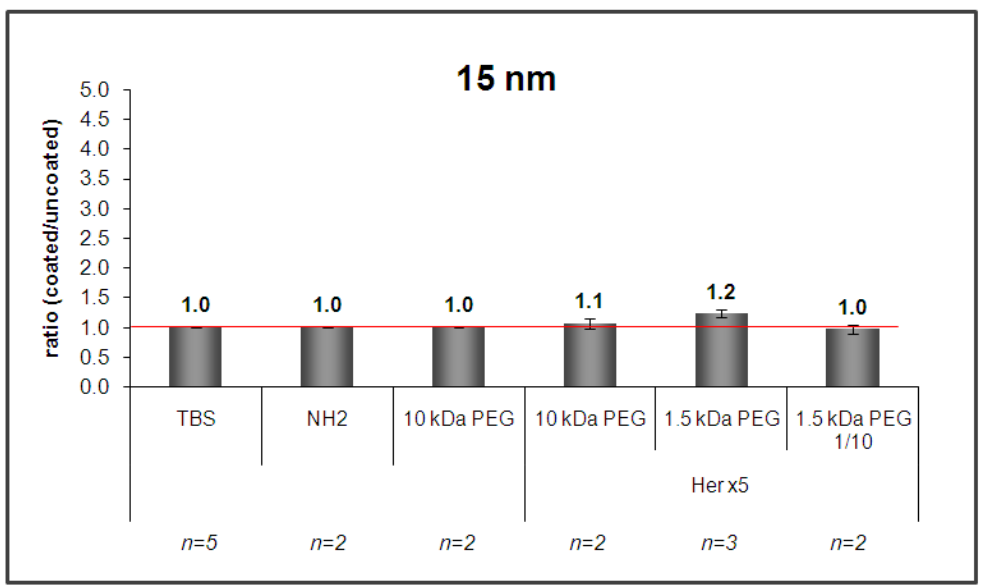

\section{Figure 29. Binding specificity of bioconjugated $15 \mathrm{~nm}$ NPs}

Signals from immunoassays with $15 \mathrm{~nm}$ NPs were measured in the Odyssey infrared imaging system. The ratio of fluorescence detected after incubation with $0.2 \mathrm{~g} / \mathrm{l} \mathrm{NPs}$ on HER2 antigen-coated and -uncoated wells, is illustrated. The control experiments, after incubation with only TBS or $\mathrm{NH}_{2}$

and PEG $10 \mathrm{kDa}$ NPs all show a ratio of 1.0. After Herceptin conjugation (Her x5), NPs with 10 and $1.5 \mathrm{kDa}$ PEG chains have a slightly increased ratio of 1.1 and 1.2, respectively. No binding of Herceptin-conjugated NPs (Her x5) is observed for NPs with PEG $1.5 \mathrm{kDa}$ chains when lower amounts of PEG (1/10) were used for coupling (ratio of 1.0). The number of independently done experiments is listed under the probes. Standard errors are illustrated as black bars. Each experiment was performed in triplicate.

Further it was tested whether bioconjugation of $25 \mathrm{~nm}$ particles with Herceptin was successful and thus specific binding of these probes to HER2 occurs. As can be seen in Figure 30A, the controls, $\mathrm{NH}_{2}-$ or PEGylated NPs (PEG $1.5 \mathrm{kDa}$ and PEG $10 \mathrm{kDa}$ ) do not specifically bind to antigen-coated wells. A weak binding to antigen-coated wells is observed after coupling of Herceptin to $25 \mathrm{~nm}$ particles with PEG $1.5 \mathrm{kDa}$ chains (ratio of 1.7) that cannot be further enhanced when the amount of antibody was increased 5-fold for coupling procedure (Her $\times 5 ; 2.4 \mathrm{nmol} / \mathrm{mg}$ PS, see section 
2.2.3.3, Table 2; ratio of 1.7). The use of PEG $10 \mathrm{kDa}$ chains leads to a reduced antigen binding after Herceptin conjugation, with a ratio of 1.4 . The bioconjugated NPs with the highest ratio and therefore best HER2 binding, PEG $1.5 \mathrm{kDa}-\mathrm{Her}$ and PEG $1.5 \mathrm{kDa}-\mathrm{Her} \times 5$ were selected for further analysis. Figure 30B, illustrates that binding of these probes to HER2-coated wells is saturated at concentrations of $1 \mathrm{~g} / \mathrm{l}$ whereas binding to uncoated wells further increases up to $2 \mathrm{~g} / \mathrm{l}(\mathrm{n}=1)$. The ratios of binding to coated and uncoated wells for both $25 \mathrm{~nm}$-Her probes (Figure 30C) are comparable high at 0.2 to $1 \mathrm{~g} / \mathrm{l}$ (ratios between 1.6 and 1.8). Therefore, $\mathrm{a}$ concentration of $0.2 \mathrm{~g} / \mathrm{l}$ was chosen for further analysis of binding specificity of NPs which was evaluated for the $25 \mathrm{~nm}$ PEG $1.5 \mathrm{kDa}$-Her x5 probe.

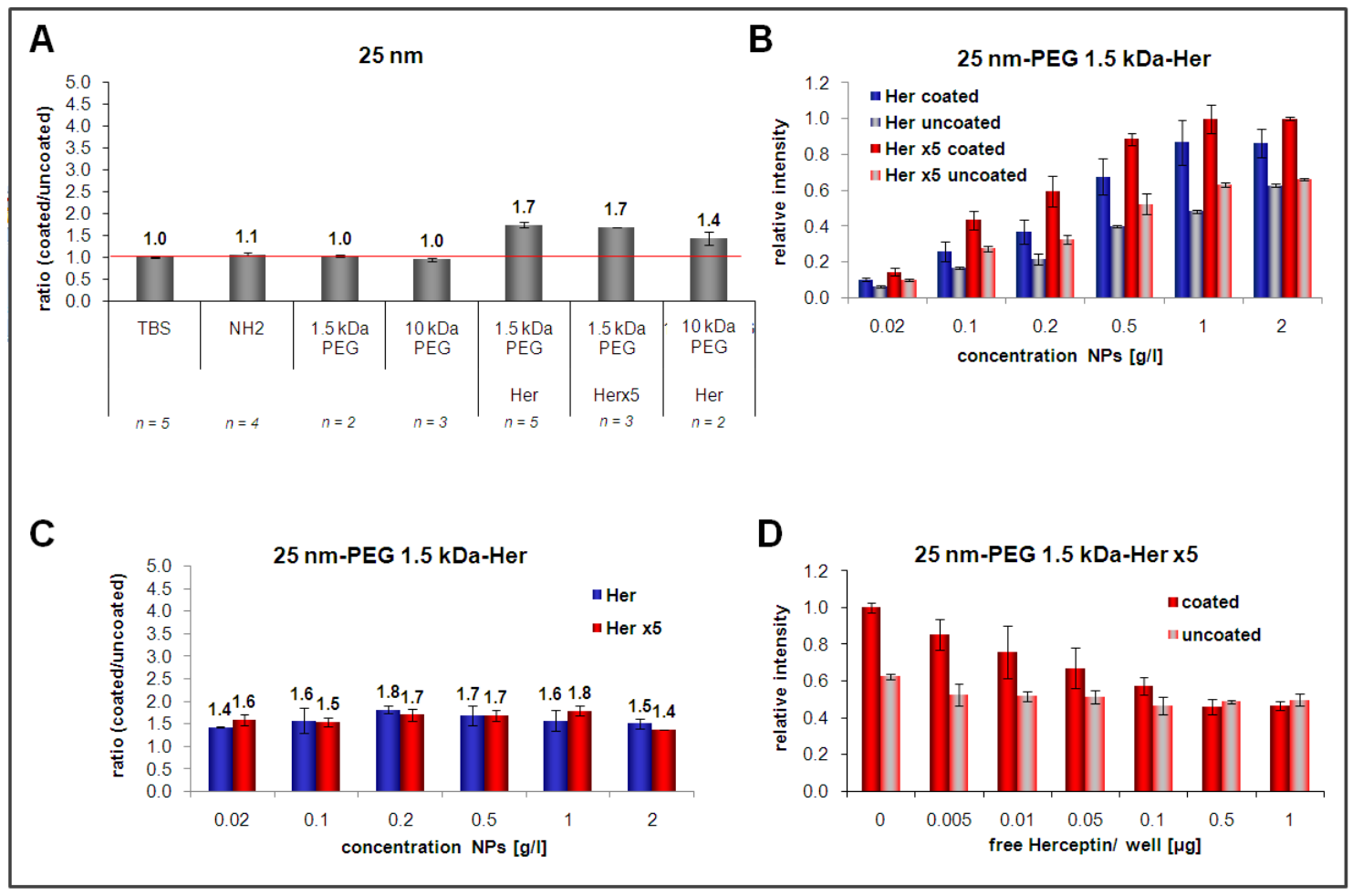

\section{Figure 30. Binding specificity of bioconjugated $25 \mathrm{~nm}$ NPs}

Signals from immunoassays with $25 \mathrm{~nm}$ NPs were measured in the Odyssey infrared imaging system. A. The ratios of signals from HER2-coated and -uncoated wells incubated with $0.2 \mathrm{~g} / \mathrm{l}$ of NPs are illustrated. The control experiments, after incubation with only TBS or $\mathrm{NH}_{2}$, PEG $1.5 \mathrm{kDa}$, and $10 \mathrm{kDa}$ NPs all show low ratios (of 1.1 and 1.0). Slightly higher ratios are observed for the Herceptin-conjugated NPS PEG $1.5 \mathrm{kDa}$ Her and -Her x5 (ratio of 1.7) and PEG $10 \mathrm{kDa}$-Her (ratio of 1.4). The number of independently done experiments is listed under the probes. B. Relative intensities of $25 \mathrm{~nm}$ PEG $1.5 \mathrm{kDa}-\mathrm{Her}$ and $-\mathrm{Her} \times 5 \mathrm{NPs}$ incubated on HER2-coated and -uncoated wells at concentrations of 0.02 to $2 \mathrm{~g} / \mathrm{l}$ are shown. C. The respective ratios of signals from coated and uncoated wells for both probes are illustrated. D. Relative intensities of $25 \mathrm{~nm}$ PEG $1.5 \mathrm{kDa}-\mathrm{Her} \times 5 \mathrm{NPs}(\mathrm{n}=2)$ incubated on HER2-coated and -uncoated wells at concentrations of $0.2 \mathrm{~g} / \mathrm{l}$. Free Herceptin was added in increasing amounts (0.005 to $1 \mu \mathrm{g})$ to the wells. Black bars indicate standard errors in $\mathbf{A}, \mathbf{C}$. and standard deviations in $\mathbf{B}, \mathbf{D}$. Each experiment was performed in triplicate. 
Figure 30D shows, that binding of the probe to HER2 is specifically occurring via interaction of Herceptin and the antigen as this binding can be "blocked" by addition of increasing amounts of free Herceptin $(n=2)$. After co-incubation with $0.5 \mu \mathrm{g}$ Herceptin per well, binding of $25 \mathrm{~nm}$ PEG $1.5 \mathrm{kDa}-\mathrm{Her}$ x 5 NPs can be completely reduced to the level of unspecific binding to uncoated wells.

In Figure $31 \mathrm{~A}$, the ratios of signals from antigen-coated and -uncoated wells after incubation with $100 \mathrm{~nm}$ particles are illustrated. NPs without bioconjugation show no binding to the antigen $\left(\mathrm{NH}_{2}\right.$, ratio of 1.0 and PEG $1.5 \mathrm{kDa}$, ratio of 1.1$)$. In contrary, the Herceptin-conjugated NPs with PEG $1.5 \mathrm{kDa}$ chains show a 4.7-fold increased ratio which is even slightly higher (ratio of 4.8) when the double amount of Herceptin was used for bioconjugation (Her $\times 2 ; 0.96 \mathrm{nmol} / \mathrm{mg}$ PS, see section 2.2.3.3, Table 2 ). Antigen binding is reduced when PEG $10 \mathrm{kDa}$ chains were used as a linker for Herceptin to the NPs, which is in agreement with the results observed for the $25 \mathrm{~nm}$ NPs (see Figure 30A). Here, a ratio of 2.9 and 2.7 was calculated for two independently produced $100 \mathrm{~nm}$-PEG $10 \mathrm{kDa}-\mathrm{Her}$ probes. This effect can be explained by the differences in number of Herceptin molecules per $100 \mathrm{~nm}$ particle (listed in section 2.2.3.3., Table 3): The 100-PEG $1.5 \mathrm{kDa}-\mathrm{Her}$ and $-\mathrm{Her} \times 2$ probes bear $\sim 70$ and 100 Herceptin molecules on the surface of one particle, whereas the 100-PEG $10 \mathrm{kDa}$-Her probes bear only $\sim 10$ antibodies per NP. Therefore, the 100 nm-PEG $1.5 \mathrm{kDa}-\mathrm{Her}$ and -Her x2 NPs, showing the highest ratios, were selected for further studies. As illustrated in Figure 31B, the binding of PEG $1.5 \mathrm{kDa}-\mathrm{Her}$ to the antigen is saturated at $0.2 \mathrm{~g} / \mathrm{l}$ whereas binding of PEG $1.5 \mathrm{kDa}-\mathrm{Her} \times 2$ increases up to $1 \mathrm{~g} / \mathrm{l}$ as does the unspecific binding for both probes to the uncoated wells $(n=1)$. Analysis of the ratio of signals from coated and uncoated wells (Figure $31 \mathrm{C}$ ) reveals best ratios for the PEG $1.5 \mathrm{kDa}-\mathrm{Her}$ and -Her x2 probes at $0.1 \mathrm{~g} / \mathrm{l}$ (ratio of 4.8 and 4.1 ) and $0.2 \mathrm{~g} / \mathrm{l}$ (ratio of 4.6 and 4.2). Exemplarily, the binding specificity is shown for the PEG $1.5 \mathrm{kDa}-H e r$ NPs at $0.2 \mathrm{~g} / \mathrm{l}$ in Figure 31D. Binding of NPs to HER2 can be decreased via addition of increasing amounts of free Herceptin $(n=2)$. After coincubation with $0.5 \mu \mathrm{g}$ Herceptin per well the signal from bound NPs is comparable low to the unspecific signal deriving from uncoated wells.

In summary, the highest binding specificity to HER2 was achieved after Herceptinconjugation to the $100 \mathrm{~nm}$ NPs, whereas bioconjugated 25 and $15 \mathrm{~nm}$ NPs showed weak or almost no binding to HER2, respectively. For the bioconjugated $25 \mathrm{~nm}$ as well as $100 \mathrm{~nm}$ particles stronger binding to HER2 was observed when short PEG chains $(1.5 \mathrm{kDa})$ were linked to the NP-surface compared to longer PEG chains (10 $\mathrm{kDa}$ ). 


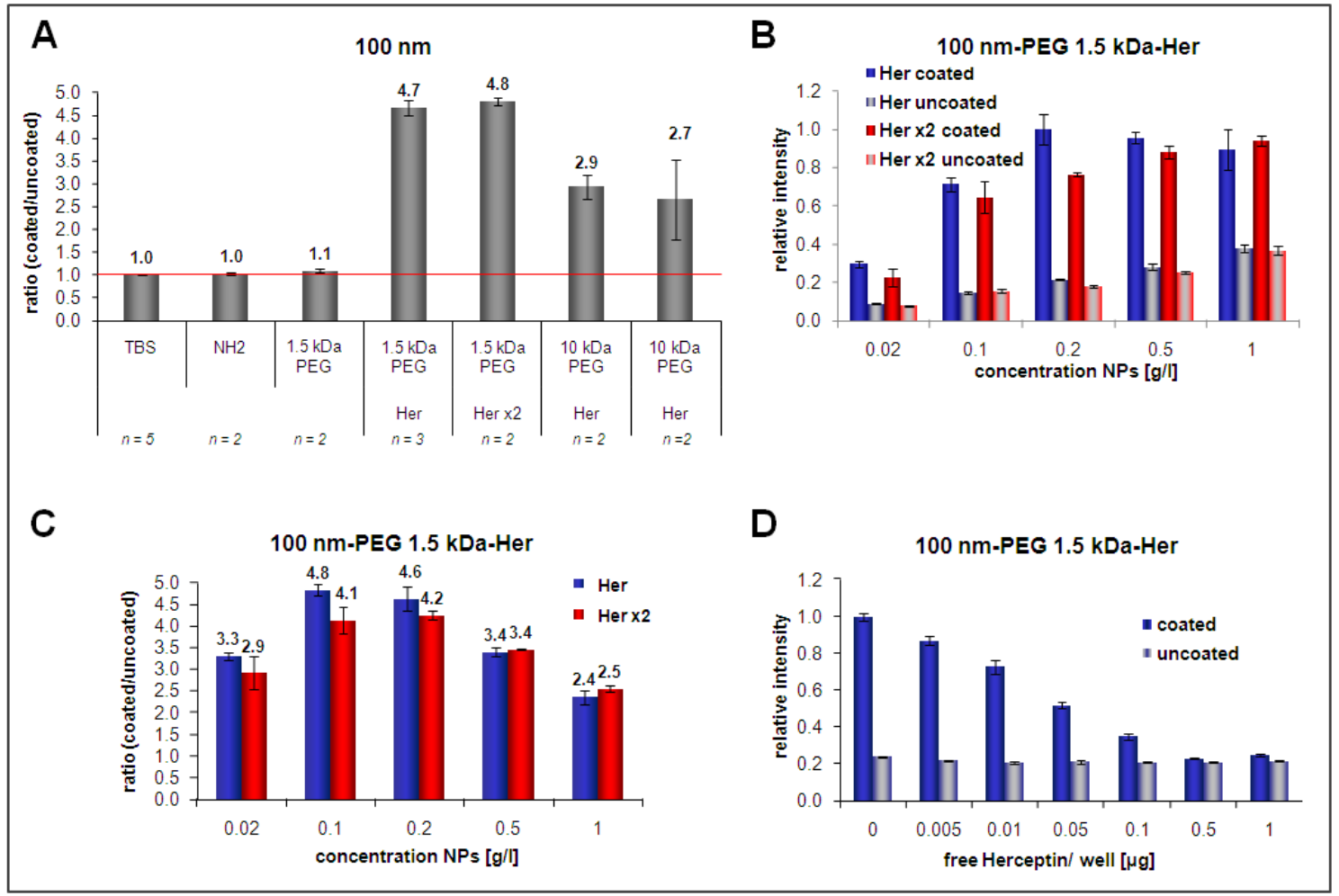

Figure 31. Binding specificity of bioconjugated $100 \mathrm{~nm}$ NPs

Signals from immunoassays with $100 \mathrm{~nm}$ NPs were measured in the Odyssey infrared imaging system. A. The ratios of signals from HER2-coated and -uncoated wells incubated with $0.2 \mathrm{~g} / \mathrm{l}$ of NPs are illustrated. The control experiments, after incubation with only TBS or $\mathrm{NH}_{2}$ and PEG $1.5 \mathrm{kDa}$ NPs, all show low ratios (of 1.1 and 1.0). An increase in specific binding can be observed for $1.5 \mathrm{kDa}$ PEG-Her and -Her x2 (ratio of 4.7 and 4.8, respectively). Use of $10 \mathrm{kDa}$ PEG chains reduces binding of Herceptin-conjugated NPs to HER2 (ratio of 2.9 and 2.7 for two independently generated probes). The number of independently done experiments is listed under the respective probes. B. Relative intensities of $100 \mathrm{~nm}-1.5 \mathrm{kDa}-\mathrm{Her}$ and -Her x2 NPs incubated on coated and uncoated wells at concentrations of 0.02 to $1 \mathrm{~g} / \mathrm{l}$ are shown. C. The respective ratios of signals from coated and uncoated wells for both probes are illustrated. D. Relative intensities of $100 \mathrm{~nm}$-PEG $1.5 \mathrm{kDa}$-Her NPs $(n=2)$ incubated on HER2-coated and -uncoated wells at concentrations of $0.2 \mathrm{~g} / \mathrm{l}$. Free Herceptin was added in increasing amounts (0.005 to $1 \mu \mathrm{g})$ to the wells. Black bars indicate standard errors in $\mathbf{A}, \mathbf{C}$. and standard deviations in $\mathbf{B}, \mathbf{D}$. Each experiment was performed in triplicate.

\subsubsection{NPs show no influence on cell viability}

As 100-PEG 1.5 kDa-Her and -Her x2 NPs showed the highest binding specificity to HER2, these probes were selected for further analyses of tumor cell binding specificity in vitro and in vivo.

Since, potentially cytotoxic effects of NPs, especially to non-cancerous cells had to be excluded, a cell viability assay was performed on 3T3 mouse fibroblasts. Fibroblasts were incubated for $24 \mathrm{~h}$ with 0.01 to $2 \mathrm{~g} / \mathrm{l} 100 \mathrm{~nm}$ NPs and the amount of viable cells, proportional to the amount of formazan was measured by absorption at 450 
$\mathrm{nm}$. Figure 32 shows that $100 \mathrm{~nm}-\mathrm{NH}_{2}$ NPs (blue columns; $\mathrm{n}=3$ ) as well as Herceptin-conjugated NPs (PEG 1.5 kDa-Her, green columns, $n=2$; PEG 1.5 kDaHer $x 2$, red columns, $n=1$ ) had no effect on cell viability when compared to untreated cells $(0 \mathrm{~g} / \mathrm{l}$, grey column, $\mathrm{n}=3)$ with a confidence of $90 \%$ (red lines). Control samples without cells, containing only medium (medium, grey column; $n=1$ ) showed a low absorption, far outside the $90 \%$ confidence interval of untreated 3 T3 cells.

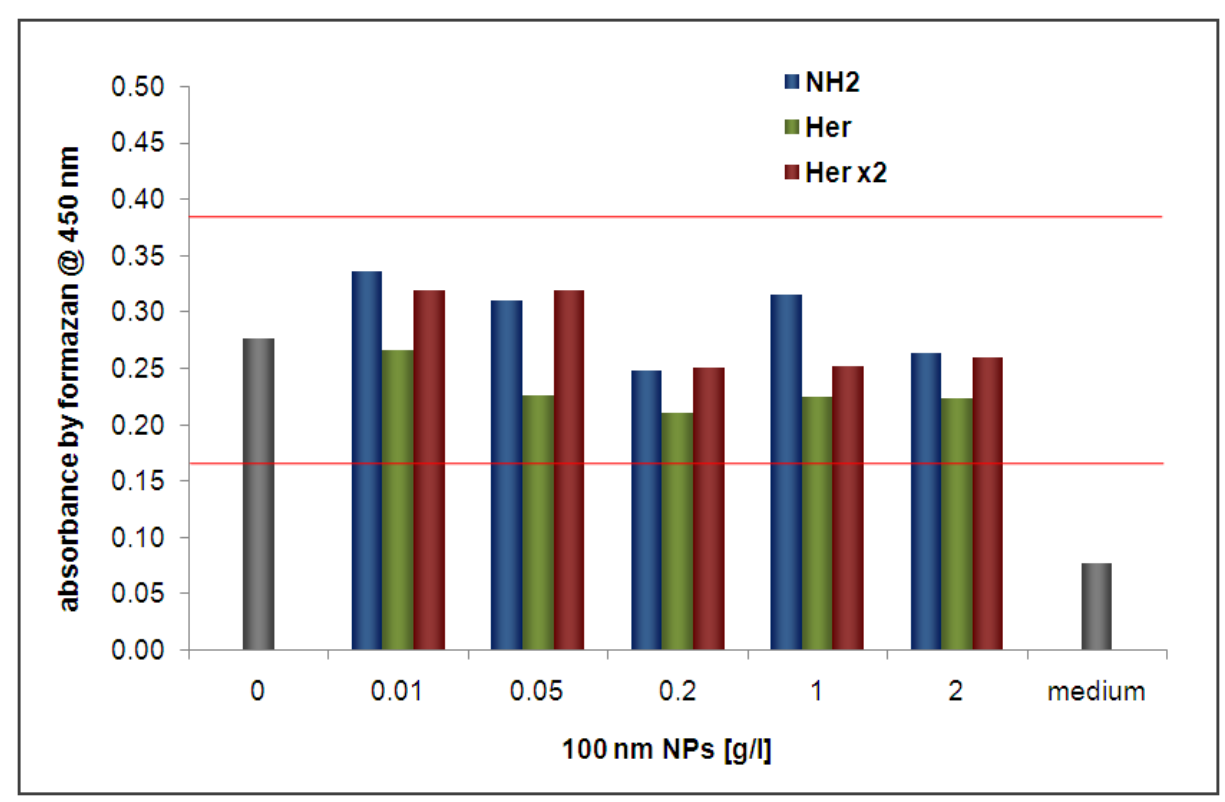

\section{Figure 32. NPs do not decrease cell viability}

3T3 mouse fibroblasts were incubated with 0.01 to $2 \mathrm{~g} / \mathrm{l}$ of $100 \mathrm{~nm} \mathrm{NPs}$ for $24 \mathrm{~h}$ and cell viability was analyzed via WST-1 assay and measurement of absorption at $450 \mathrm{~nm}$. To exclude absorption effects by the Itrybe-loaded NPS, absorption was measured before addition of WST-1 and subtracted from absorption after WST-1 treatment. The mean values of the absorption by formazan are illustrated for untreated cells $(0 \mathrm{~g} / \mathrm{l} \mathrm{NPs} ; \mathrm{n}=3$ ) and for medium only (grey column; $\mathrm{n}=1$ ) as well as for cells incubated with $100 \mathrm{~nm}$ NPs. Incubation with NPs is shown in blue columns for $100 \mathrm{~nm}-\mathrm{NH}_{2}(\mathrm{n}=3)$, in green columns for $100 \mathrm{~nm}$ PEG $1.5 \mathrm{kDa}$-Her $(n=2)$, and in red columns for $100 \mathrm{~nm}$-PEG $1.5 \mathrm{kDa}$-Her $\times 2(n=1)$. All experiments were performed in triplicate. The $90 \%$ confidence interval of untreated cells is depicted in red lines.

In summary, NPs at concentrations up to $2 \mathrm{~g} / \mathrm{l}$ incubated for $24 \mathrm{~h}$ do not affect cell viability of mouse fibroblasts. 


\subsubsection{Binding specificity of bioconjugated NPs to breast tumor cells in vitro}

As 100-PEG $1.5 \mathrm{kDa}-\mathrm{Her}$ and -Her $\mathrm{x} 2 \mathrm{NPs}$ showed strong binding to the HER2 antigen in immunoassays (see section 3.3.2, Figure 31), they were analyzed for their binding capacity to KPL-4 and MDA-MB-231 tumor cells by fluorescence microscopy, in comparison to controls, the $100-\mathrm{NH}_{2}$ and -PEG $1.5 \mathrm{kDa}$ NPs.

Specific binding of Herceptin-conjugated NPs to HER2-positive KPL-4 cells was observed, whereas the controls revealed also some unspecific binding. Figure 33 shows, that strongest fluorescence is detected from HER2-positive, KPL-4 cells after incubation with $100 \mathrm{~nm}$-PEG $1.5 \mathrm{kDa}$-Her NPs (Her) whereas signals are low after incubation of the same cell type with the unspecific probe, $100 \mathrm{~nm}$ - PEG $1.5 \mathrm{kDa}$ NPs (PEG). Incubation of both probes with HER2-negative, MDA-MB-231 cells also revealed comparable low fluorescence $(n=3)$. The differences of specific and unspecific binding to cells decreased with increasing incubation times: Whereas the differences of fluorescence of KPL-4 cells incubated with Her to the control samples (PEG on KPL-4 as well as Her and PEG on MDA-MB-231 cells) are relatively high after $30 \mathrm{~min}$ (Figure 33A) and $1 \mathrm{~h}$ (Figure 33B), unspecific uptake of the control samples in cells increased after $3 \mathrm{~h}$ (Figure 33C) and $5 \mathrm{~h}$ of incubation (Figure 33D).

The binding and/or uptake of $100 \mathrm{~nm}$-PEG $1.5 \mathrm{kDa}$-Her NPs on KPL-4 cells over time is illustrated in Figure 34. Itrybe-derived signals continuously increased from $30 \mathrm{~min}$ to $5 \mathrm{~h}$ after incubation of the probe at $37^{\circ} \mathrm{C}$ (Figure $34 \mathrm{~A} ; \mathrm{n}=3$ ). At $4{ }^{\circ} \mathrm{C}$ incubation (Figure 34B; $n=1$ ), no fluorescence can be detected on cells using the same exposure time $(10 \mathrm{~ms})$. Nevertheless, an increase of Itrybe-derived fluorescence over time is also detected at $4^{\circ} \mathrm{C}$ with a higher exposure time of $20 \mathrm{~ms}$ (Figure 34C), however, cell binding and/or uptake of NPs is lower compared to incubation at $37^{\circ} \mathrm{C}$. The distribution of NPs on cells incubated at $37{ }^{\circ} \mathrm{C}$ (Figure $34 \mathrm{~A}$ ) and at $4{ }^{\circ} \mathrm{C}$ (Figure $34 \mathrm{C}$ ) is relatively equal over time as no differences in location of NPs, e.g. predominantly membrane bound probe at early time points in comparison to internalized probe after longer incubation times, are observed. NPs seem to localize inside the cells after very short incubation times (Figure 33 and Figure 34). 


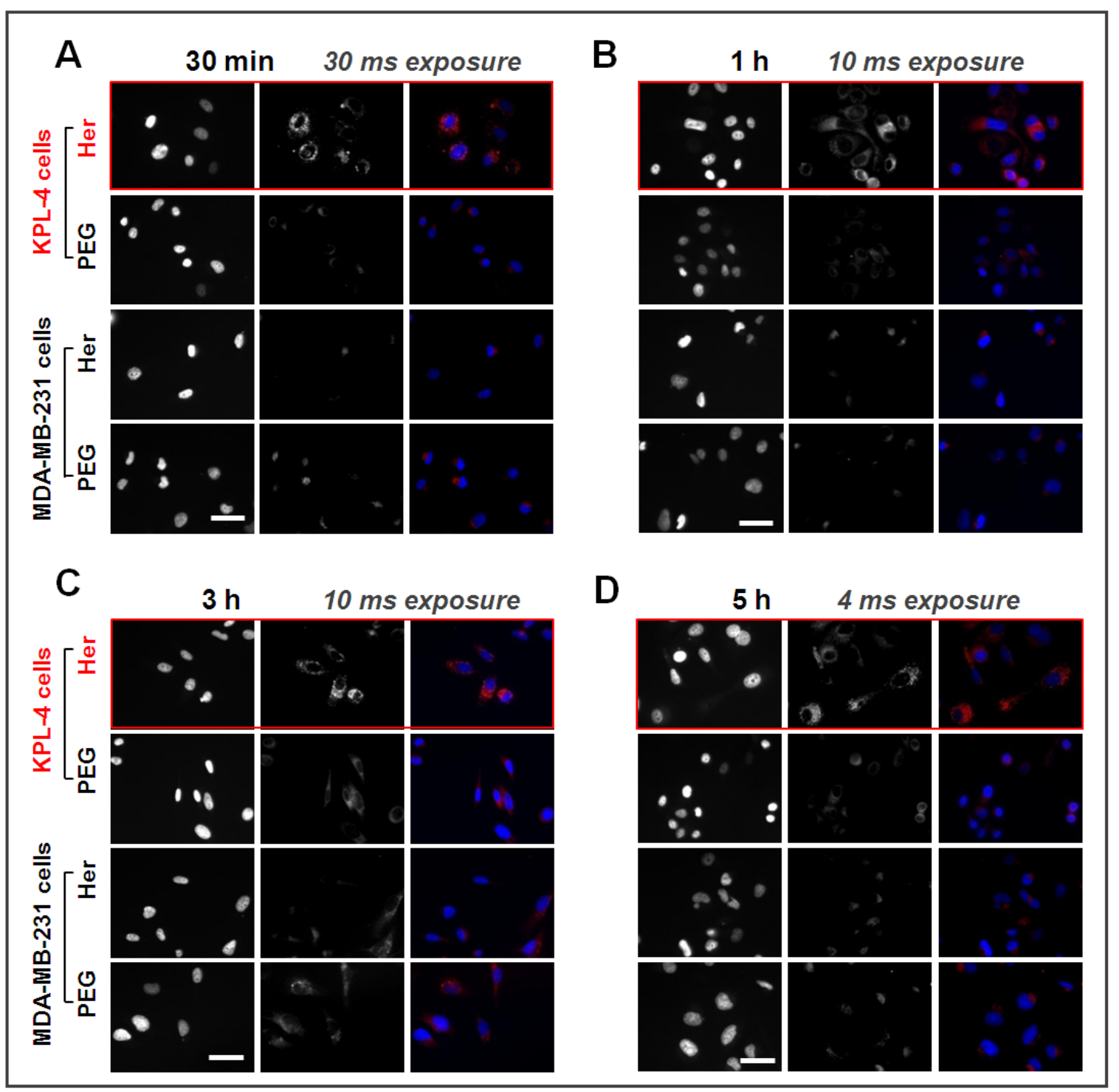

Figure 33. Increased binding/uptake of Herceptin-conjugated NPs to HER2-positive cells in vitro

Cells were seeded on glass slides and incubated for $30 \mathrm{~min}, 1,3$, and $5 \mathrm{~h}$ at $37^{\circ} \mathrm{C}$ with $1 \mathrm{~g} / \mathrm{l}$ Itrybe-loaded $100 \mathrm{~nm}$ NPs and examined under a fluorescence microscope with an $x 40$ objective. For each box, on the left, counter stain of cell nuclei with Hoechst 33342 and in the middle probe-derived signals are illustrated. Merged images of the cell nuclei (blue) and the probe (red) can be seen on the right. The exposure time used for detection of Itrybe was $30 \mathrm{~ms}$ after $30 \mathrm{~min}, 10 \mathrm{~ms}$ after 1 and $3 \mathrm{~h}$, and $4 \mathrm{~ms}$ after $5 \mathrm{~h}$ of incubation. A,B. After (A) $30 \mathrm{~min}$ and (B) $1 \mathrm{~h}$ of incubation strongest fluorescence is deriving from KPL-4 cells treated with $100 \mathrm{~nm}$-PEG $1.5 \mathrm{kDa}-\mathrm{Her}$ (Her). The control probe, PEG $1.5 \mathrm{kDa}$ (PEG) on KPL-4 cells as well as both probes on MDA-MB-231 cells reveal low fluorescence signals. C,D. When incubated (C) for $3 \mathrm{~h}$ and (D) $5 \mathrm{~h}$ with $100 \mathrm{~nm}$ NPs, still strongest fluorescence is deriving from $100 \mathrm{~nm}$-Her on KPL-4 cells but signals from $100 \mathrm{~nm}-\mathrm{PEG}$ on KPL-4 cells and from both probes on MDA-MB-231 cells are slightly increased, compared to A. and B. Representative results of three independently performed experiments are shown. Bars represent $50 \mu \mathrm{m}$. 


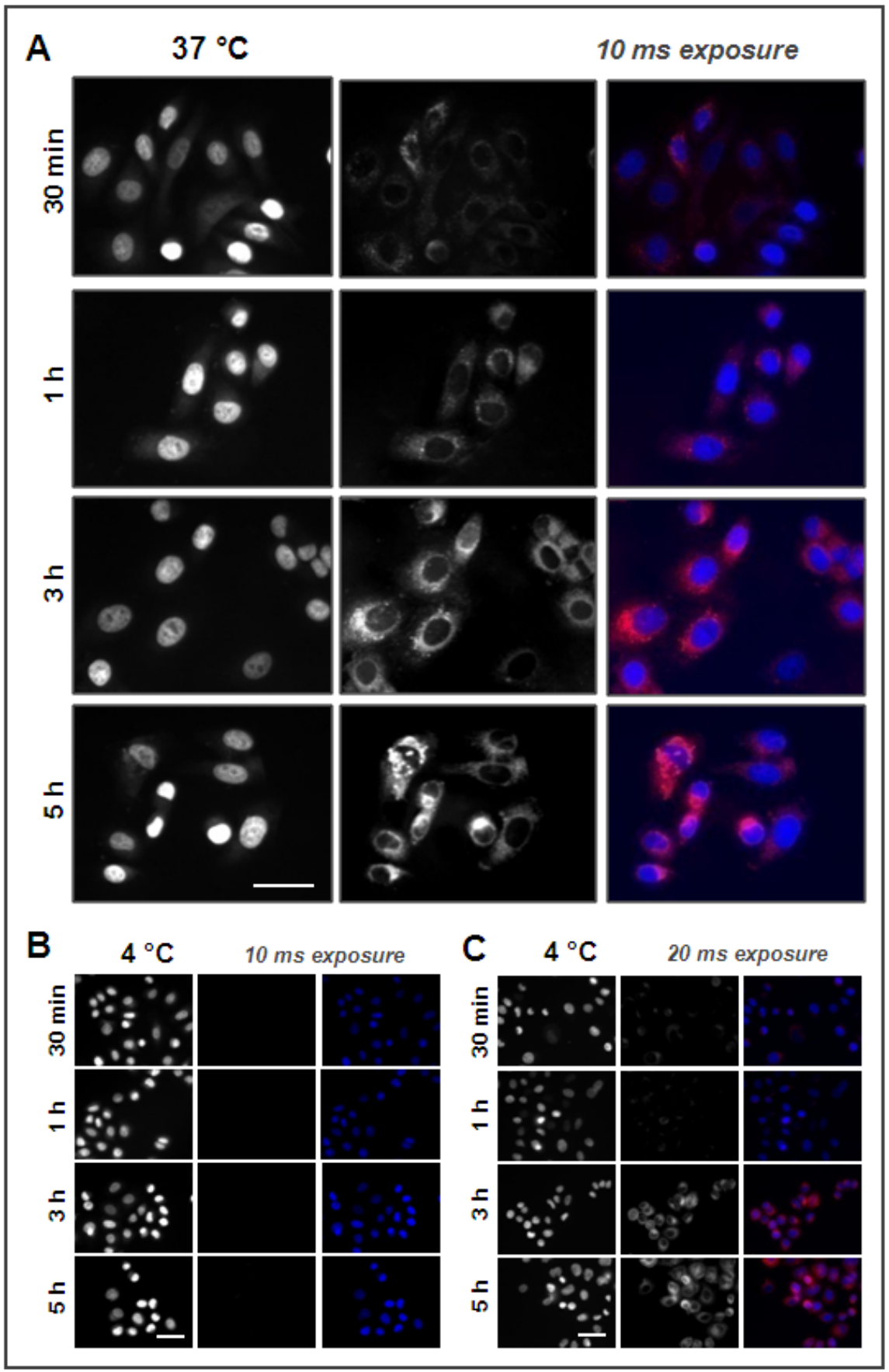

Figure 34. Increased binding/uptake of Herceptin-conjugated $100 \mathrm{~nm}$ NPs in HER2-positive cells over time at 37 and $4^{\circ} \mathrm{C}$

$\mathrm{KPL}-4$ cells were incubated for $30 \mathrm{~min}, 1,3$, and $5 \mathrm{~h}$ at $37{ }^{\circ} \mathrm{C}$ and $4{ }^{\circ} \mathrm{C}$ with $1 \mathrm{~g} / \mathrm{l}$ Itrybe-loaded $100 \mathrm{~nm}$ PEG $1.5 \mathrm{kDa}$-Her NPs and examined under a fluorescence microscope with an $\mathrm{x} 40$ objective. For each box, on the left, counter stain of cell nuclei with Hoechst 33342 and in the middle, probe-derived signals are illustrated. Merged images of the cell nuclei (blue) and the probe (red) can be seen on the right. A. Itrybederived fluorescence on cells, detected with a constant exposure time of $10 \mathrm{~ms}$, continuously increases over time at $37{ }^{\circ} \mathrm{C}$. Representative results of three independently done experiments are shown. B. No fluorescence signals on cells can be detected after incubation at $4{ }^{\circ} \mathrm{C}(n=1)$ at the same exposure time as in A., of $10 \mathrm{~ms}$. C. When using a higher exposure time of $20 \mathrm{~ms}$, increased Itrybe-derived signals on cells can be detected over time. Bars represent $50 \mu \mathrm{m}$. 
The binding specificity of Herceptin-conjugated NPs was further verified on KPL-4 and MDA-MB-231 tumor sections (two tumors of each type) that were incubated with different $100 \mathrm{~nm}$ probes. As illustrated in Figure 35, $100 \mathrm{~nm}-\mathrm{PEG} 1.5 \mathrm{kDa}-\mathrm{Her}$ x2 NPs ( $n=2$ ) show strong binding to KPL-4 tumors (Figure $35 \mathrm{~A} ; n=2$ ), compared to the control probes, $100 \mathrm{~nm}-\mathrm{PEG} 1.5 \mathrm{kDa}$ and $100 \mathrm{~nm}-\mathrm{NH}_{2}$ (Figure 35B,C; $\mathrm{n}=2$ ).

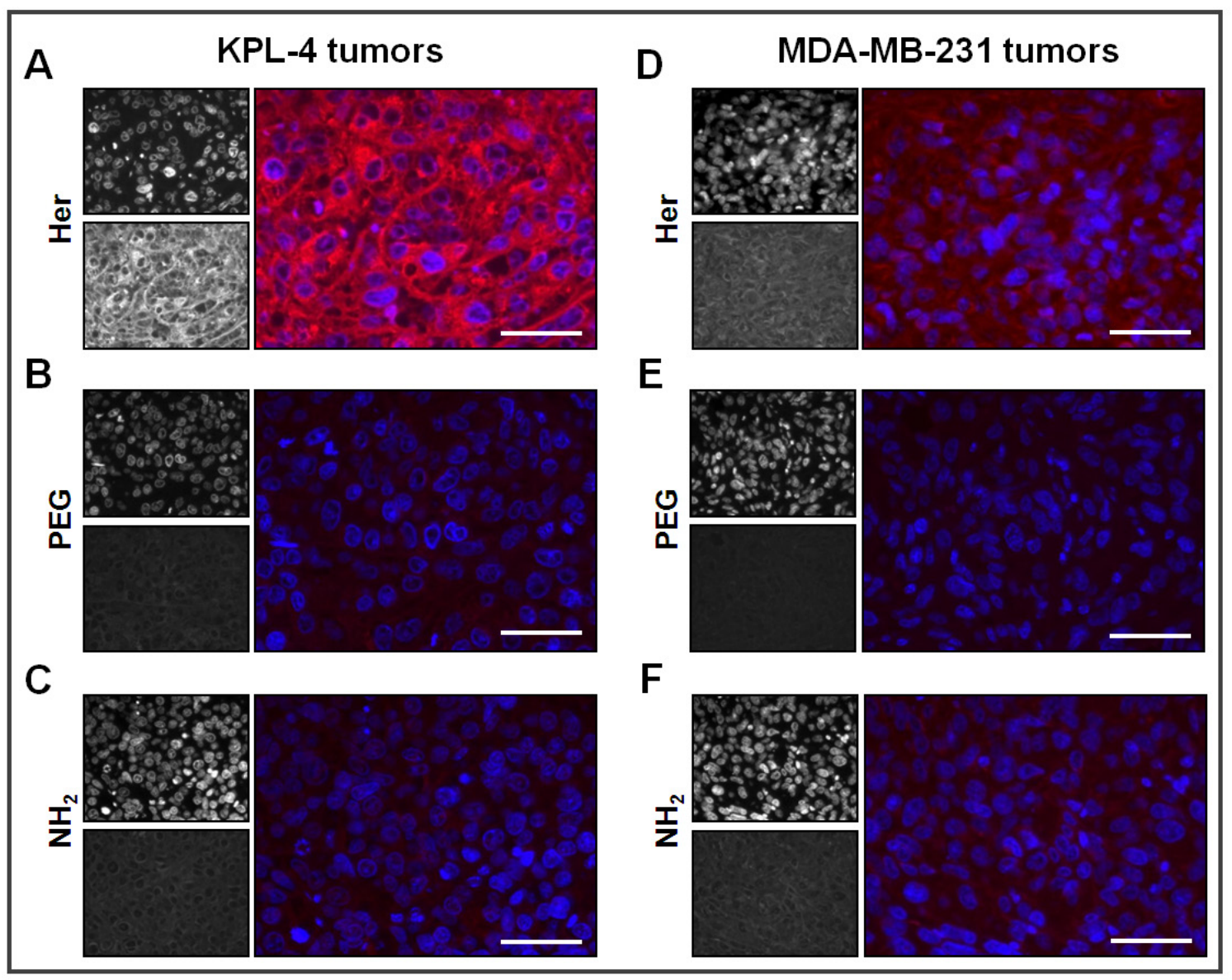

Figure 35. Ex vivo fluorescence experiments demonstrate binding specificity of Herceptinconjugated NPs to HER2-positive tumors

Paraffin fixed KPL-4 and MDA-MB-231 tumor sections were incubated with $0.1 \mathrm{~g} / \mathrm{l}$ Itrybe-loaded NPs and examined under a fluorescence microscope with a $\times 40$ objective. For each box, on the left upper side, counter stain of cell nuclei with DAPI and on the left lower side, probe-derived signals are illustrated. Merged images of the cell nuclei (blue) and the probe (red) can be seen on the right. A-C. Representative results of two different KPL-4 tumors incubated with (A) $100 \mathrm{~nm}$-PEG $1.5 \mathrm{kDa}-\mathrm{Her} \times 2(\mathrm{Her} ; \mathrm{n}=2)$ show strong fluorescence in comparison to KPL-4 tumors incubated with (B) $100 \mathrm{~nm}-\mathrm{PEG} 1.5 \mathrm{kDa}$ (PEG; $\mathrm{n}=1$ ), or (C) $100 \mathrm{~nm}-\mathrm{NH}_{2}\left(\mathrm{NH}_{2} ; \mathrm{n}=2\right)$. D-F. Representative results of two different MDA-MB-231 tumors incubated with (D) 100 nm-PEG $1.5 \mathrm{kDa}-H e r \times 2$ (Her; $\mathrm{n}=1$ ), (E) $100 \mathrm{~nm}-\mathrm{PEG} 1.5 \mathrm{kDa}$ (PEG; $\mathrm{n}=1$ ), or (F) $100 \mathrm{~nm}-\mathrm{NH}_{2}\left(\mathrm{NH}_{2} ; \mathrm{n}=1\right)$ reveal lower fluorescence compared to $A$. The exposure time for detection of NPs was $140 \mathrm{~ms}$. Bars represent $50 \mu \mathrm{m}$. 
Weak fluorescence could be observed in MDA-MB-231 tumors treated with $100 \mathrm{~nm}$ PEG $1.5 \mathrm{kDa}$-Her $x 2 \mathrm{NPs}$ (Figure 35D) that was slightly higher compared to both tumor types incubated with the control probes (Figure 35B-C,E-F).

Taken together, Herceptin-conjugated $100 \mathrm{~nm}$ NPs are functional on breast tumor cells, as they showed increased binding/uptake to HER2-positive KPL-4 cells and tumors. In contrary, PEGylated or $\mathrm{NH}_{2}$-modified NPs showed lower binding to $\mathrm{KPL}-4$ cells and -tumors as did all NP probes to HER2-negative, MDA-MB-231 cells and tumors.

\subsubsection{In vivo detection of Itrybe-loaded NPs}

The suitability of fluorescence deriving from Itrybe-loaded NPs to be detected in vivo in tumor tissue and at other sites of the mice was investigated. For this purpose, KPL-4 and MDA-MB-231 tumor-bearing nude mice received i.v. injections of different Itrybe-loaded NPs and were imaged in vivo and ex vivo at diverse time points. Due to the broad absorption spectra of Itrybe-loaded NPs (see section 3.3.1, Figure 27), imaging was performed at two different $\lambda_{\text {ex }}$ of 635 and $670 \mathrm{~nm}$ and fluorescence was detected at $\lambda_{\mathrm{em}}$ above $700 \mathrm{~nm}(\mathrm{n}=9)$.

Figure 36A shows representative in vivo images of a KPL-4 tumor-bearing nude mouse before and 1 to $24 \mathrm{~h}$ after $i . v$. injection of $400 \mu \mathrm{g} 100 \mathrm{~nm}$-PEG $1.5 \mathrm{kDa}-\mathrm{Her}$ at $\lambda_{\text {ex }} 635 \mathrm{~nm}(\mathrm{n}=2)$. Autofluorescence (white circle) was assessed in prescans of mice before treatment. High fluorescence in the liver is seen shortly after NP-injection up to $24 \mathrm{~h}$ (white ellipse after $1 \mathrm{~h}$ ). However, no tumor signals were detected within this time (white circle). The same mouse imaged at $\lambda_{\text {ex }} 670 \mathrm{~nm}$, is illustrated in Figure 36B. Here, autofluorescence is lower in the prescan but unspecific signals in the stomach (arrow) and gut (white ellipse) appear higher. NP-derived fluorescence in the liver was also observed but appears weaker than at $\lambda_{\text {ex }} 635 \mathrm{~nm}$ (Figure 36A). This can be explained by the better excitability of Itrybe at $\lambda_{\text {ex }} 635 \mathrm{~nm}$, compared to $\lambda_{\mathrm{ex}} 670 \mathrm{~nm}$ (see Figure 27 in section 3.3.1). Ex vivo scans of excised organs of the same mouse $24 \mathrm{~h}$ after probe injection are depicted in Figure 36C,D. Here, the highest signals can be seen in the liver at $\lambda_{\mathrm{ex}} 635 \mathrm{~nm}$ (Figure 36C) as well as at $\lambda_{\mathrm{ex}}$ $670 \mathrm{~nm}$ (Figure 36D) compared to low signals at both wavelengths in the gut, stomach, kidneys, heart, spleen, lung, and tumor. In comparison to excitation at $\lambda_{\mathrm{ex}}$ $635 \mathrm{~nm}$, Itrybe-derived fluorescence in the liver is weaker at $\lambda_{\mathrm{ex}} 670 \mathrm{~nm}$ but unspecific signals from the GI tract are slightly higher, which is in agreement with the in vivo measurements. 


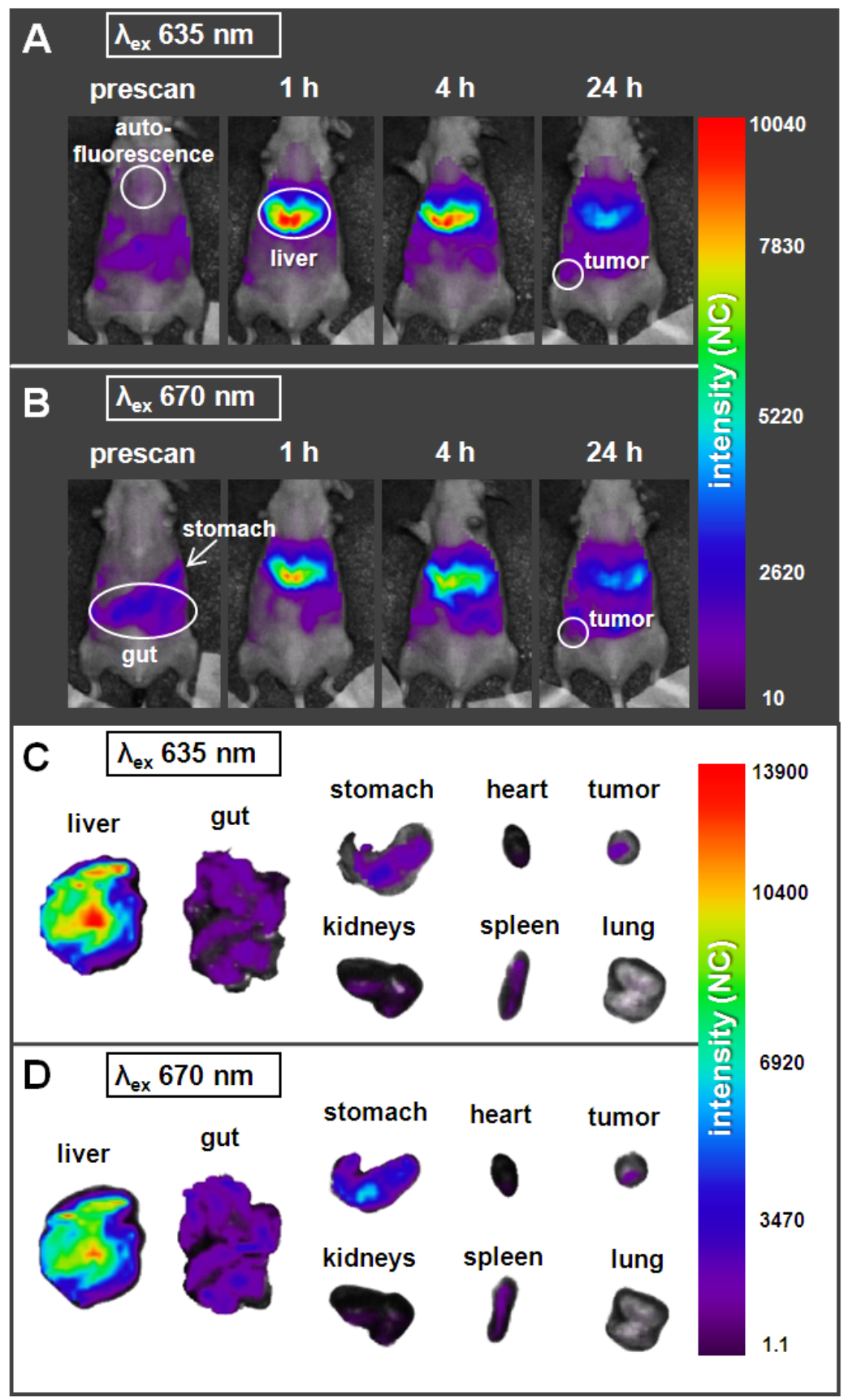

Figure 36. In vivo and ex vivo detection of NP-derived fluorescence at different wavelengths

Fluorescence intensity scans taken by the Optix MX2 before (prescan) and after i.v. injection of $400 \mu \mathrm{g}$ $100 \mathrm{~nm}$-PEG $1.5 \mathrm{kDa}$-Her NPs in nude mice bearing KPL-4 tumors in the right abdominal mammary gland $(n=2)$. A. A representative mouse in a ventral position imaged before and 1,4 , and $24 \mathrm{~h}$ after probe application at $\lambda_{\mathrm{ex}} 635 \mathrm{~nm}$ and $\lambda_{\mathrm{em}}$ above $700 \mathrm{~nm}$. Autofluorescence in the prescan over the lung area is illustrated with a white circle. No accumulation of NPs in the tumor (white circle, $24 \mathrm{~h}$ ) but high liver signals (white ellipse, $1 \mathrm{~h}$ ) are observed after probe application. B. The same mouse shown in A. imaged at $\lambda_{\mathrm{ex}} 670 \mathrm{~nm}$ and $\lambda_{\mathrm{em}}$ above $700 \mathrm{~nm}$. In the prescan autofluorescence is lower compared to $A$. but unspecific signals from the stomach (arrow) and gut (white ellipse) are higher. C. Ex vivo scans of excised organs of the mouse shown in A. and B. at $\lambda_{\text {ex }} 635 \mathrm{~nm}, 24 \mathrm{~h}$ after NP-injection confirm in vivo observations. D. The same organs seen in C. imaged at $\lambda_{\mathrm{ex}}$ of $670 \mathrm{~nm}$. 
In summary, Itrybe-loaded NPs can be excited at $\lambda_{\text {ex }} 635 \mathrm{~nm}$ but also at $\lambda_{\text {ex }} 670 \mathrm{~nm}$ and be detected well above $\lambda_{\mathrm{em}} 700 \mathrm{~nm}$. Following injection of NPs, probe-derived fluorescence cannot be detected in the tumor, but strong signals are observed in the liver. Due to the higher fluorescence of Itrybe at $\lambda_{\mathrm{ex}} 635 \mathrm{~nm}$ making autofluorescence appear negligible, this excitation wavelength was used for all following in vivo and ex vivo scans performed in the Optix MX2.

In order to illustrate the brightness of Itrybe-loaded NPs and its detection limit for tumors in vivo, different concentrations (100 to $0.8 \mu \mathrm{g}$ ) of $15 \mathrm{~nm}-\mathrm{PEG} 10 \mathrm{kDa}$ NPs were injected s.c. in mice at different sites $(n=2) .15 \mathrm{~nm}$ particles were selected due to their lowest fluorescence intensity per mg PS compared to other NPs (see Table 11). As illustrated in Figure 37, fluorescence intensities of NPs after s.c. injection (Figure 37A,B; white circles) are well detectable even at an amount of 1.6 $\mu \mathrm{g}$, as NPs are still 4-times more fluorescent (Figure 37C; 1,200 NC) than the autofluorescence (Figure 37B,D; red circle; $327 \mathrm{NC}$ ). In comparison, fluorescence of $1.6 \mu \mathrm{g}$ of NPs injected in the MDA-MB-231 tumor (Figure 37C; $813 \mathrm{NC}$ ) is slightly weaker, as only 2.5 -fold higher than the autofluorescence.

As a result, Itrybe-derived fluorescence can be detected in very low amounts in vivo, although fluorescence is lower within the tumor compared to s.c. injections near the surface of the mouse. Therefore, Itrybe-loaded NPs are suitable for in vivo tumor imaging in mice. 


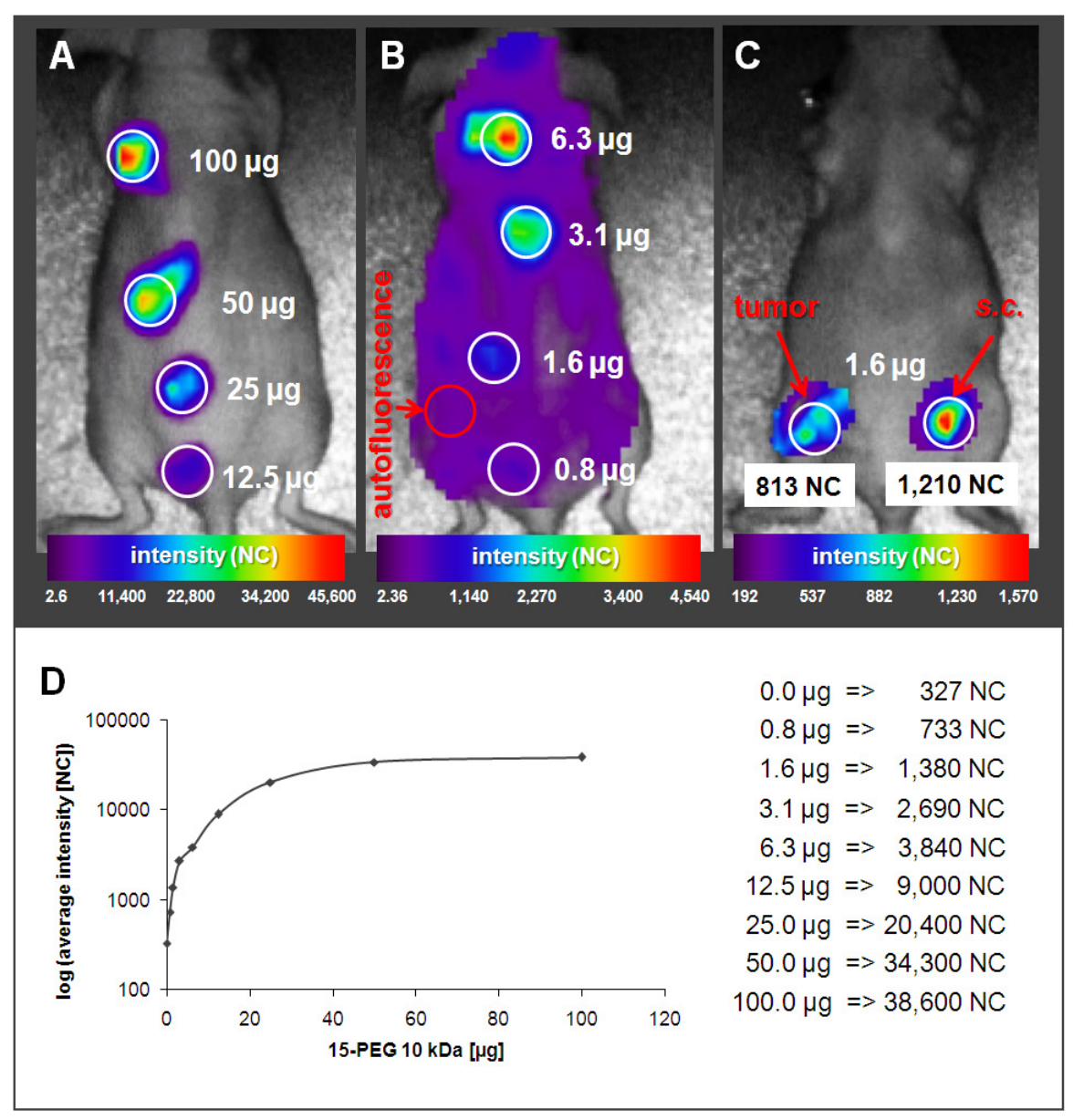

\section{Figure 37. In vivo detection of Itrybe-derived fluorescence}

Fluorescence intensity scans taken by the Optix MX2 directly after injection of different amounts of $15 \mathrm{~nm}$ PEG $10 \mathrm{kDa}$ NPs solved in $30 \mu \mathrm{l} \mathrm{NaCl}$. The areas of probe injections are indicated with white circles. A. Mouse in a dorsal view with s.c. injections of $100,50,25$, and $12.5 \mu \mathrm{g}$ NPs $(n=2)$. B. Mouse in a dorsal view with s.c. injections of $6.3,3.1,1.6$, and $0.8 \mu \mathrm{g} \mathrm{NPs}(n=2)$. Autofluorescence (red circle) was determined in an area outside the gut and stomach C. A MDA-MB-231 tumor-bearing mouse in a ventral position injected with $1.6 \mathrm{\mu g}$ NPs i.t. (red arrow) and s.c. (red arrow). Note that different intensity scales are shown in A-C. D. The log of the average intensity after s.c. injection of 100 to $0.8 \mu \mathrm{g} 15 \mathrm{~nm} \mathrm{NPs}(A, B)$ and of the autofluorescence of the mouse $(0.0 \mu \mathrm{g}$ NPs) is illustrated.

\subsubsection{Biodistribution of NPs}

In order to compare the in vivo behavior of NPs depending on different sizes and surface modifications, biodistribution studies were performed in healthy nude mice. As NPs seem to be captured in the liver rapidly after injection (see section 3.3.5, Figure 36), mice were analyzed at early time points, from 6 min to $4 \mathrm{~h}$ after injection of $\mathrm{NH}_{2}$ and PEGylated NPs of different sizes $(n=28)$.

As illustrated in Figure 38, exemplarily for a mouse injected with $25 \mathrm{~nm}$-PEG $10 \mathrm{kDa}$ $(n=4)$, NPs accumulate in the liver (white ellipse) already 6 min after injection. Therefore, fluorescence is especially analyzed over the liver and compared to 
fluorescence measured in the background of mice, representing the amount of circulating unbound NPs in the blood. An area over the hindlimb of mice (in a ventral position) was selected as a background region (white circle), since no unspecific accumulation of NPs is expected here. After injection of NPs, only a marginal increase in fluorescence is detected in the background (white circle) indicating a fast elimination of NPs from the blood pool.

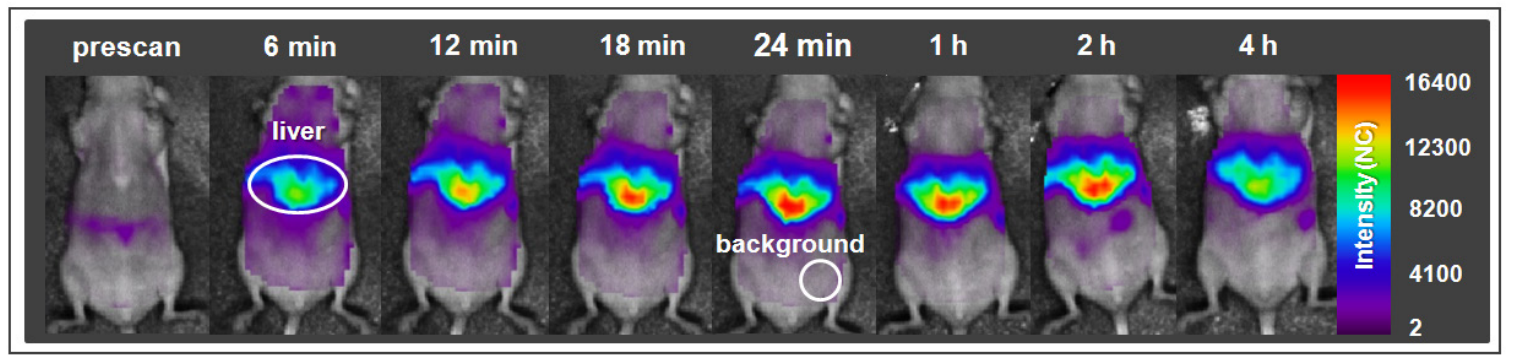

Figure 38. Biodistribution of NPs in healthy mice over time

Fluorescence intensity maps of healthy, tumor free, female nude mice scanned in vivo in the Optix MX2 before (prescan) and $6 \mathrm{~min}$ to $4 \mathrm{~h}$ after injection of diverse NPs $(\mathrm{n}=28)$. Exemplarily, a representative mouse before and after injection of $400 \mu \mathrm{g} 25 \mathrm{~nm}$-PEG $10 \mathrm{kDa}$ NPs is illustrated $(n=4)$. High liver signals can be seen rapidly after probe application (white ellipse) whereas background fluorescence (white circle) remains low.

In Figure 39 average fluorescence intensities in liver (Figure 39A-C) and background regions (Figure 39D-F) of healthy nude mice treated with different NPs are illustrated before and 6 min to $4 \mathrm{~h}$ after treatment ( $n=4$; see also Figure 38 ). In order to eliminate signals deriving from autofluorescence of the mouse, the average fluorescence in the liver and background of the prescans of each mouse was subtracted from the respective regions after probe injection.

Note that intensities are not comparable between mice treated with NPs of varying sizes $(15,25$, and $100 \mathrm{~nm})$ since the fluorescence intensity of the injected probe varies dependent on the NP size. As illustrated in Table 11, per mg PS, the $25 \mathrm{~nm}$ NPs are 3-fold more fluorescent and the $15 \mathrm{~nm}$ particles are 5-fold less fluorescent compared to the $100 \mathrm{~nm}$ NPs. 


\section{Table 11. Fluorescence intensities of NPs of different sizes}

Total fluorescence intensities of NP probes in $200 \mu \mathrm{N} \mathrm{NaCl}$ were measured in the Optix MX2 prior to i.v. injection in mice. Measurements and calculations were performed for the PEGylated NPs (100 nm-PEG 1.5 $\mathrm{kDa}, 25 \mathrm{~nm}$-PEG $10 \mathrm{kDa}$, and $15 \mathrm{~nm}$-PEG $10 \mathrm{kDa}$ ).

\begin{tabular}{|c|c|c|c|}
\hline probe & $\mathbf{1 0 0} \mathbf{~ n m}$ & $\mathbf{2 5} \mathbf{~ n m}$ & $\mathbf{1 5} \mathbf{~ n m}$ \\
\hline $\begin{array}{c}\text { injected amount } \\
{[\mathrm{mg}]}\end{array}$ & 0.4 & 0.4 & 1 \\
\hline PI [NC] & $1,900,000$ & $5,630,000$ & 790,000 \\
\hline relative* PI/mg & 1.0 & 3.0 & 0.2 \\
\hline
\end{tabular}

$\mathrm{PI}=$ Total fluorescence intensity of the injected probe

* relative to $100 \mathrm{~nm}$ NPS

As summarized in Figure 39A-C, NPs with increasing size accumulate slower in the liver: Fluorescence of $15 \mathrm{~nm}$ NPs already peaks $\sim 6$ min (Figure 39A), signals of 25 $\mathrm{nm}$ NPs peak $\sim 30 \mathrm{~min}$ (Figure 39B), and fluorescence of $100 \mathrm{~nm}$ NPs peaks $\sim 1$ to $2 \mathrm{~h}$ after injection (Figure 39C). Furthermore, PEGylation of probes influences their accumulation in the liver, as 25 and $100 \mathrm{~nm}$ NPs demonstrate decreased liver signals after PEGylation whereas PEGylated $15 \mathrm{~nm}$ NPs show increased signals when compared to the $\mathrm{NH}_{2}$-modified particles (Figure 39A-C). All probes show low fluorescence in the background with a maximal intensity within the first view minutes after injection of NPs that decreases over time (Figure 39D-F). Only the 100-PEG 1.5 kDa NPs (Figure 39F) revealed a well measurable and stable background signal over time (from 30 min to $4 \mathrm{~h}$ ). In contrary, PEGylation of the 15 and $25 \mathrm{~nm}$ NPs had no impact on the prolongation of their blood circulation time (Figure 39D,E).

Healthy nude mice treated with NPs (see Figure 38 and 39) were sacrificed $4.5 \mathrm{~h}$ after injection and excised organs were scanned in the Optix MX2 ex vivo. Probederived fluorescence in the organs is displayed according to the presumable size of the injected NPs from the probe with the highest to the lowest diameter $(100 \mathrm{~nm}$ PEG $1.5 \mathrm{kDa}>100 \mathrm{~nm}-\mathrm{NH}_{2}>25 \mathrm{~nm}$-PEG $10 \mathrm{kDa}>25 \mathrm{~nm}$-PEG $1.5 \mathrm{kDa}>25 \mathrm{~nm}-$ $\mathrm{NH}_{2}>15 \mathrm{~nm}$-PEG $10 \mathrm{kDa}>15 \mathrm{~nm}-\mathrm{NH}_{2}$ ). To exclusively illustrate NP-derived signals, the autofluorescence from untreated organs $(n=4)$ was subtracted from the respective organs from mice that received NPs. To allow comparability of intensities from different probes, the average fluorescence intensity in particular organs is illustrated relative to the total intensity of all organs (liver, lung, heart, spleen, and kidneys) of mice treated with the same probe $(n=4)$. 


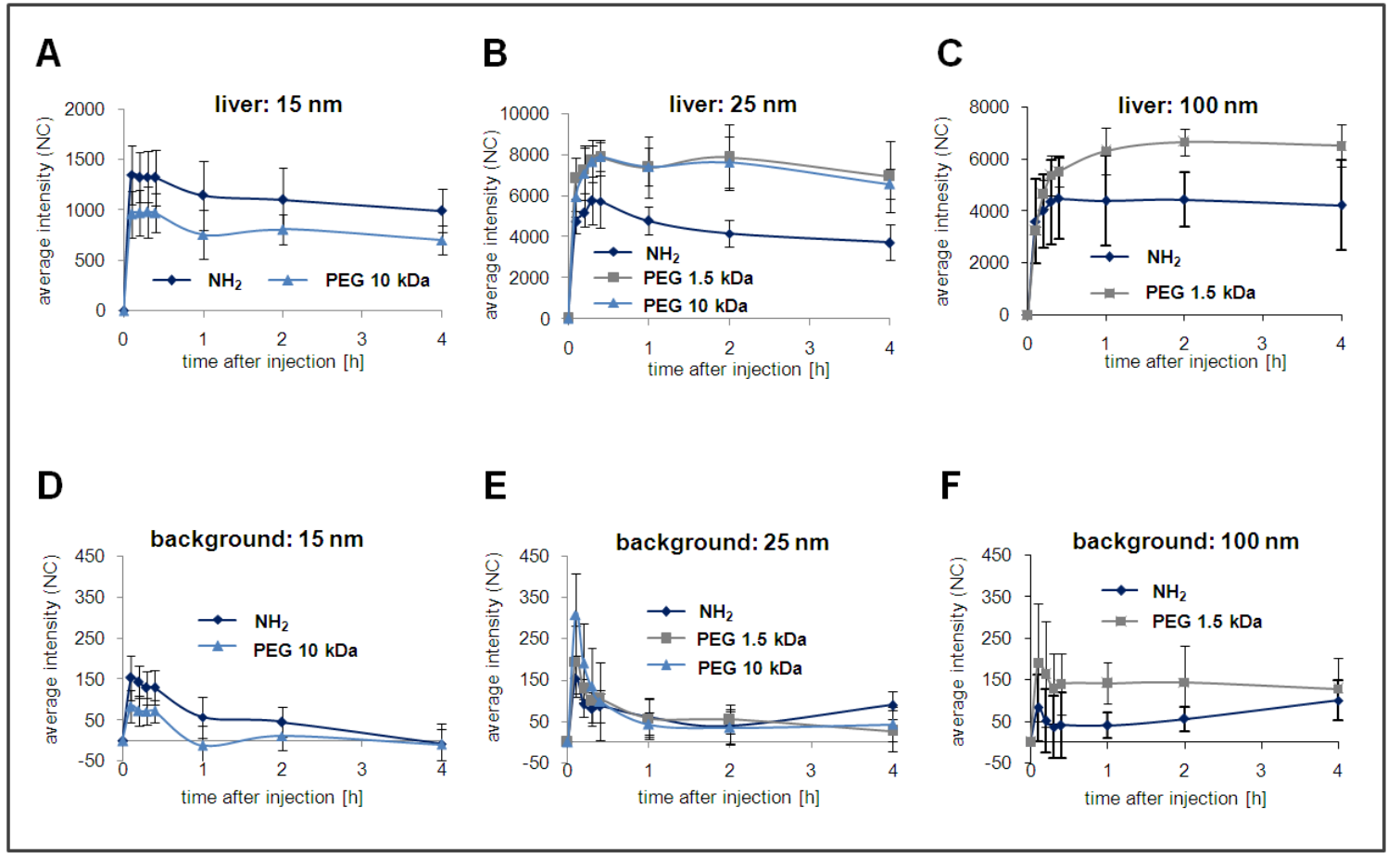

Figure 39. NP distribution in liver and background of healthy mice in vivo over time

Average fluorescence intensities in liver $(\mathbf{A}-\mathbf{C})$ and background regions (D-F) of female healthy nude mice treated with distinct NPs are shown before and $6 \mathrm{~min}$ to $4 \mathrm{~h}$ after probe injection $(n=4)$. Mice received $(A, D) 1 \mathrm{mg}$ of $15 \mathrm{~nm} \mathrm{NPs},(B, E) 400 \mu \mathrm{g}$ of $25 \mathrm{~nm}$, or $(C, F) 400 \mu \mathrm{g}$ of $100 \mathrm{~nm} \mathrm{NPs}$. The average autofluorescence in the liver and background of the prescans of each mouse is subtracted from the respective regions after probe injection. Liver signals after injection of (A) $15 \mathrm{~nm}-\mathrm{NH}_{2}$ and -PEG $10 \mathrm{kDa}$, (B) $25 \mathrm{~nm}-\mathrm{NH}_{2}$, -PEG $1.5 \mathrm{kDa}$, and $-10 \mathrm{kDa}$, as well as (C) $100 \mathrm{~nm}-\mathrm{NH}_{2}$ and -PEG $1.5 \mathrm{kDa}$ are shown. Corresponding background signals after injection of (D) $15 \mathrm{~nm}-\mathrm{NH}_{2}$ and -PEG $10 \mathrm{kDa}$, (E) $25 \mathrm{~nm}-\mathrm{NH}_{2}$, -PEG $1.5 \mathrm{kDa}$, and -10 kDa, as well as (F) $100 \mathrm{~nm}-\mathrm{NH}_{2}$ and -PEG $1.5 \mathrm{kDa}$ are illustrated. Mean values of 4 mice are shown. Standard deviations are indicated as black bars.

Figure $40 \mathrm{~A}$ illustrates that all probes accumulate in the liver to a high extend after i.v. injection. For example, $25 \mathrm{~nm}$ NPs show highest liver uptake with up to $90 \%$ (for the $25 \mathrm{~nm}$-PEG $10 \mathrm{kDa}$ probe) whereas lung, heart, spleen, and kidneys show comparable low fluorescence (Figure 40B). The second highest fluorescence after NP injection is seen in the spleen. Here, the $100 \mathrm{~nm}-\mathrm{NH}_{2}$ particles show the strongest fluorescence, with $9 \%$ and a slightly decreasing tendency with a decrease in size of NPs. In contrary, highest fluorescence in the kidneys was found for the smallest (15 $\mathrm{nm}$ ) NPs with $6 \%$ whereas the larger probes show lower fluorescence of 2 to $3 \%$. Fluorescence in the heart was low for all probes $(1.4 \%)$. 


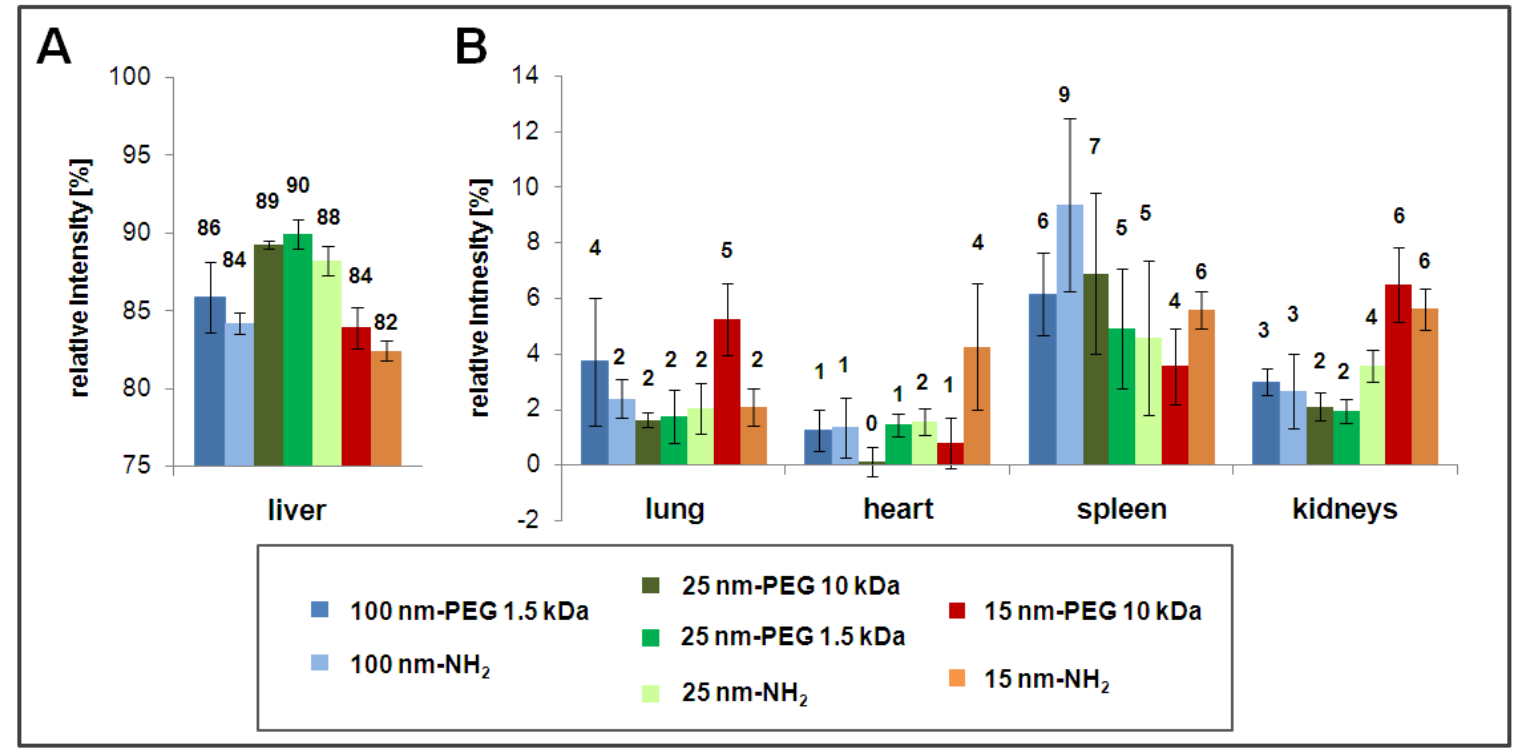

Figure 40. Biodistribution of NPs by ex vivo analysis

Healthy nude mice injected with different NPs were sacrificed after $4.5 \mathrm{~h}$ and organs were scanned in the Optix MX2 ( $\mathrm{n}=4$ ). Mice received $1 \mathrm{mg}$ of $15 \mathrm{~nm}$ or $400 \mu \mathrm{g}$ of 25 or $100 \mathrm{~nm} \mathrm{NPs,} \mathrm{with} \mathrm{NH}_{2}$ or PEG (1.5 and $10 \mathrm{kDa})$ surface groups. Autofluorescence from the respective untreated organs $(n=4)$ is subtracted from the organs of treated mice. The relative fluorescence intensity of average signals over the respective organs is illustrated. This is calculated under the assumption that liver, lung, heart, spleen, and kidneys make up $100 \%$ of the fluorescence. A. Relative fluorescence intensity in the liver is high compared to other organs. B. The spleen shows second highest uptake of NPs. In the kidneys, fluorescence increases after injection of $15 \mathrm{~nm}$ NPs.

Taken together, biodistribution studies in mice illustrate a rapid and high accumulation of all probes in the liver compared to the spleen, kidneys, lung, and heart. Ex vivo, $15 \mathrm{~nm}$ NPs showed the lowest liver accumulation but the highest signals in the kidney. With an increase in NP size, liver uptake and excretion seems to be slower over time. Blood circulation times for all NPs were low, showing peak intensity rapidly after injection, but are slightly enhanced after applying the PEGylated 100 nm NPs.

\subsubsection{NPs accumulate in MDA-MB-231 breast tumors}

In KPL-4 or MDA-MB-231 tumor-bearing mice injected with Herceptin-conjugated or PEGylated 15, 25, and $100 \mathrm{~nm}$ particles no NP-derived fluorescence could be detected in the tumors in vivo in whole-body scans up to $24 \mathrm{~h}$ (see Figure 36A,B; $\mathrm{n}=$ 19). Accumulation of NPs in a MDA-MB-231 tumor was also not observed in vivo up to $96 \mathrm{~h}$ after injection of $400 \mu \mathrm{g} 100 \mathrm{~nm}$ NPs with surface carboxyl-groups (-COOH) (data not shown).

Furthermore, tumors of mice treated with $1 \mathrm{mg}$ of $15 \mathrm{~nm}$ NPs or with $400 \mu \mathrm{g}$ of 100 and $25 \mathrm{~nm}$ NPs were excised $24 \mathrm{~h}$ after injection and scanned ex vivo $(\mathrm{n}=2)$. Figure 
$41 \mathrm{~A}, \mathrm{~B}$ shows the "total fluorescence intensities in the tumor normalized to the tumor volume" (TIV) of KPL-4 tumors from mice treated with NPs. Compared to the average TIV of untreated mice (red line), no increase in fluorescence after NP-injection is observed. In contrary, TIVs in MDA-MB-231 tumors of mice treated with PEGylated NP show a clear increase with decreasing particle size compared to tumors of untreated mice (Figure 41C,D). Note, that untreated KPL-4 tumors (Figure 41A,B) have more than twice as high autofluorescence compared to MDA-MB-231 tumors (Figure $41 C, D$ ).

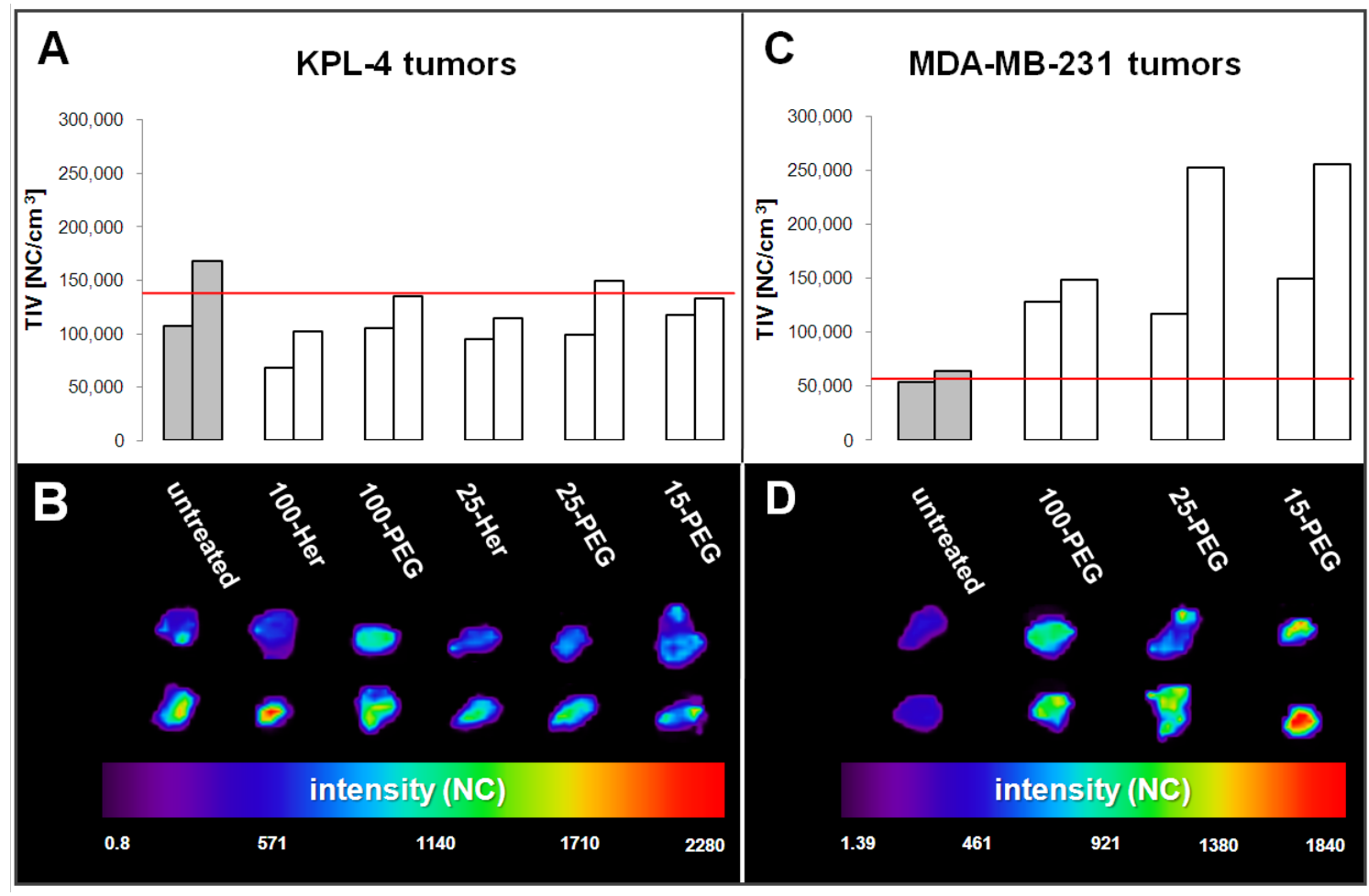

Figure 41. Passive accumulation of PEGylated NPs in MDA-MB-231 tumors ex vivo

Ex vivo analysis of excised KPL-4 and MDA-MB-231 tumors of mice $24 \mathrm{~h}$ after i.v. injection of Herceptinconjugated and PEGylated 100, 25, and $15 \mathrm{~nm} \mathrm{NPs}(n=2)$ scanned in the Optix MX2. A. Total fluorescence intensities in the tumor normalized to the tumor volume (TIV) of KPL-4 tumors before (untreated, grey columns) and after treatment (white columns) with NPs are shown. From left to right: untreated, 100 nm-PEG 1.5 kDa-Her (100-Her), 100 nm-PEG 1.5 kDa (100-PEG), 25 nm-PEG 1.5 kDa-Her (25-Her), $25 \mathrm{~nm}$-PEG $1.5 \mathrm{kDa}$ (25-PEG), and $15 \mathrm{~nm}$-PEG $10 \mathrm{kDa}$ (15-PEG). The average TIV in untreated mice is indicated with a red line. No increase in fluorescence is observed in tumors after NP-treatment compared to tumors of untreated mice. B. Fluorescence intensity scans of KPL-4 tumors corresponding to A. are illustrated. C. TIV of MDA-MB-231 tumors before (untreated; grey columns) and after treatment (white columns) with NPs are shown. From left to right: untreated, 100-PEG, 25-PEG, and 15-PEG. The average TIV in untreated mice is indicated with a red line. Increased fluorescence is measured in tumors of mice treated with NPs. D. Fluorescence intensity scans of MDA-MB-231 tumors corresponding to C. are illustrated. 
In order to obtain only the fluorescence in the tumor deriving from injected NPs, the average TIV of untreated tumors was subtracted from the average TIV after probe injection; defined as the NP-derived TIV (Table 12). As the total fluorescence intensity of the injected probe (PI) is known for the 100, 25, and $15 \mathrm{~nm}$ PEGylated NPs, the NP-derived TIV, relative to the PI was calculated (NP-derived TIV/PI). Here, $15 \mathrm{~nm}$-PEG $10 \mathrm{kDa}$ NPs show a more than 4-fold higher NP-derived TIV/PI ratio than the $100 \mathrm{~nm}$-PEG $1.5 \mathrm{kDa}$ NPs (0.18 to 0.04 ) and a 9-fold higher ratio than the 25 nm-PEG $10 \mathrm{kDa}$ NPs (0.18 to 0.02). This demonstrates that the $15 \mathrm{~nm}$ NPs show the highest fluorescence intensity in tumor tissue (normalized to the tumor volume) in relation to the intensity of the injected probe

\section{Table 12. NP-derived signals in MDA-MB-231 tumors ex vivo}

Total fluorescence intensities of NPs prior to injection in MDA-MB-231 tumor-bearing mice as well as of the excised tumors $24 \mathrm{~h}$ after injection were measured in the Optix MX2. The injected amounts of NPs were: $400 \mu \mathrm{g}$ of $100 \mathrm{~nm}$-PEG $1.5 \mathrm{kDa}$ NPs (100-PEG), $400 \mu \mathrm{g}$ of $25 \mathrm{~nm}$-PEG $10 \mathrm{kDa}$ NPs (25-PEG), and $1 \mathrm{mg}$ of $15 \mathrm{~nm}-\mathrm{PEG} 10 \mathrm{kDa}$ NPs (15-PEG).

\begin{tabular}{|c|c|c|c|}
\hline probe & 100-PEG & 25-PEG & 15-PEG \\
\hline $\begin{array}{c}\text { NP-derived TIV } \\
{\left[\mathrm{NC} / \mathrm{cm}^{3}\right]}\end{array}$ & 79,251 & 125,695 & 143,754 \\
\hline PI [NC] & $1,900,000$ & $5,630,000$ & 790,000 \\
\hline $\begin{array}{c}\text { NP-derived TIV/PI } \\
{\left[1 / \mathrm{cm}^{3}\right]}\end{array}$ & 0.04 & 0.02 & 0.18 \\
\hline
\end{tabular}

TIV = Total fluorescence intensity in the tumor normalized to the tumor volume $\mathrm{PI}=$ Total fluorescence intensity of the injected probe

In summary, ex vivo analysis revealed that Herceptin-labeled and PEGylated NPs do not specifically bind to or accumulate in HER2-positive KPL-4 tumors. However, a passive accumulation of PEGylated NPs is seen in MDA-MB-231 breast tumors, especially for the smallest $15 \mathrm{~nm}$-PEG $10 \mathrm{kDa}$ NPs.

The origin of fluorescence signals after treatment with $15 \mathrm{~nm}$-PEG $10 \mathrm{kDa}$ NPs was further assessed on the cellular level by NIR fluorescence microscopy. Therefore, tumor sections of KPL-4 and MDA-MB-231 tumors excised $24 \mathrm{~h}$ after injection of 15 nm-PEG $10 \mathrm{kDa}$ NPs were analyzed. KPL-4 tumors of untreated mice show a relatively high autofluorescence (Figure $42 \mathrm{~A} ; \mathrm{n}=2$ ) whereas no fluorescece in KPL-4 tumors of mice treated with NP can be observed (Figure 42B; $n=2$ ). Compared to untreated KPL-4 tumors, MDA-MB-231 tumors of untreated mice show lower autofluorescence (Figure 42C; $\mathrm{n}=2$ ). After treatment of mice with $15 \mathrm{~nm}$-PEG 10 kDa NPs, MDA-MB-231 tumors (Figure 42D; $n=2$ ) show fluorescence deriving from 
the tumor cells. The signal distribution within the tumor is not homogeneous with most parts showing comparably low fluorescence. In addition, more fluorescence was observed at the tumor border compared to the center (data not shown).

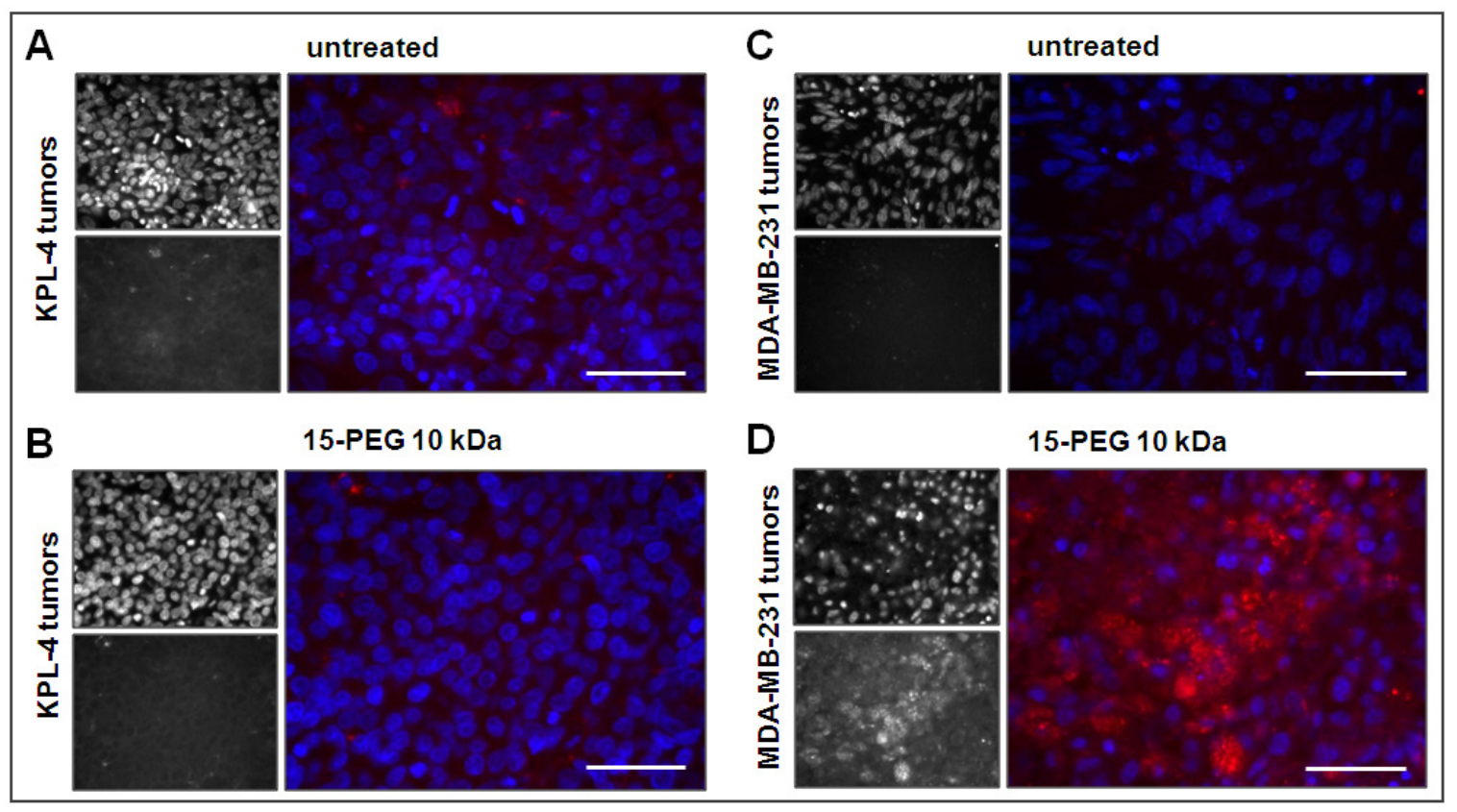

Figure 42. Detection of $15 \mathrm{~nm}$ NP-derived fluorescence in MDA-MB-231 tumor sections ex vivo

Sections of KPL-4 and MDA-MB-231 tumors of mice $24 \mathrm{~h}$ after i.v. injection of $1 \mathrm{mg} 15 \mathrm{~nm}-\mathrm{PEG} 10 \mathrm{kDa}$ NPs were examined under a fluorescence microscope with a $x 40$ objective. For each box, on the left upper side, counter stain of cell nuclei with DAPI and on the left lower side, probe-derived signals are illustrated. Merged images of the cell nuclei (blue) and the probe (red) are shown on the right. A. In untreated KPL-4 tumors $(n=2)$ some autofluorescence can be detected. B. In KPL-4 tumors of mice treated with $15 \mathrm{~nm}$ PEG $10 \mathrm{kDa}$ NPs $(n=2)$ fluorescence is not higher compared to untreated tumors in A. C. MDA-MB-231 tumors show almost no autofluorescence $(n=2)$. D. MDA-MB-231 tumors of mice treated with $15 \mathrm{~nm}$-PEG $10 \mathrm{kDa}$ NPs $(n=2)$ show fluorescence located around the tumor cells. The exposure time for detection of NPs was $800 \mathrm{~ms}$. Bars represent $50 \mu \mathrm{m}$.

Best signal detection of Itrybe-loaded NPs was observed ex vivo for the PEGylated 15 $\mathrm{nm}$ particles after injection in MDA-MB-231 tumor bearing mice. Here, illustration of tumor signals was also possible in vivo, as illustrated in Figure $43(n=2)$. As tumorderived fluorescence is relatively low after probe injection (compared to the prescan) this could only be achieved by scanning exclusively the tumor region. Thereby, the high probe-derived signals in the liver which were hampering the detection sensitivity of low fluorescent signals from the tumor are excluded (see Figure 36A for comparison). Although fluorescence in the tumor is relatively weak, the intensity in the tumor (black circle) is higher $1 \mathrm{~h}$ after injection of NPs and further increases up to $24 \mathrm{~h}$ when compared to the autofluorescence in the prescan. 


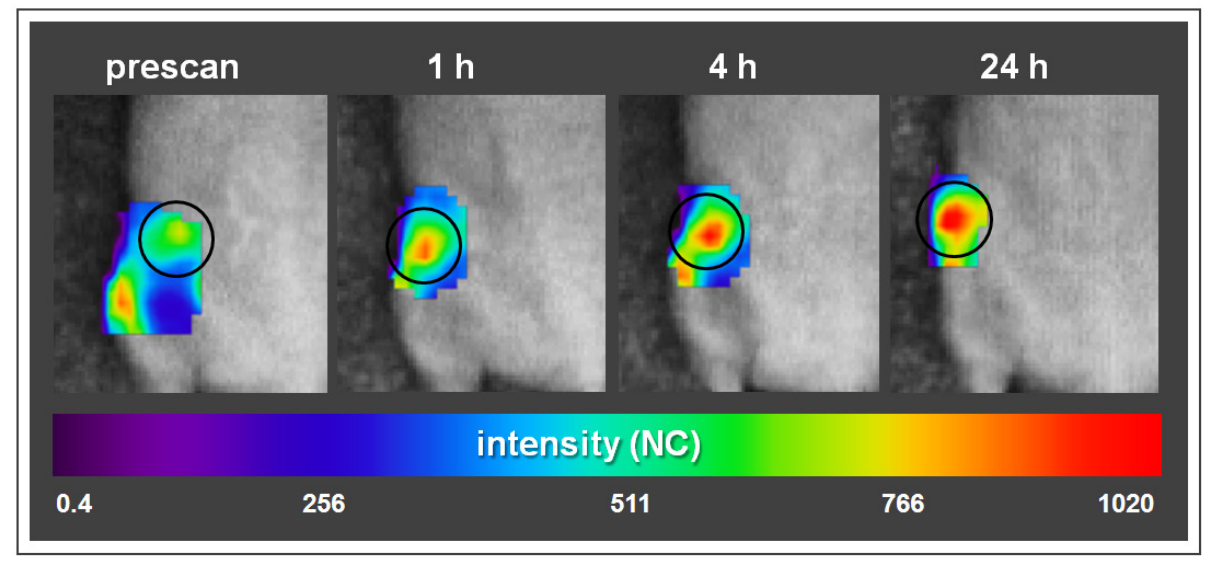

Figure 43. PEGylated $15 \mathrm{~nm}$ NPs can be detected in the tumor in vivo

In vivo fluorescence intensity scan of a MDA-MB-231 breast tumor-bearing mouse before (prescan) and 1, 4, and $24 \mathrm{~h}$ after i.v. injection of $1 \mathrm{mg} 15 \mathrm{~nm}$-PEG $10 \mathrm{kDa}$ NPs $(\mathrm{n}=2)$ performed in the Optix MX2. Only the tumor region (black circle) was scanned. After injection of NPs, fluorescence over the tumor increases over time.

In summary, tumor-bearing mice treated with NPs of all sizes with PEG- and Herceptin surface-modifications showed no NP-derived fluorescence in the tumor in vivo in whole body scans. In contrary, a closer analysis of the tumor targeting ability of NPs ex vivo revealed low but clear accumulation of NPs in MDA-MB-231 breast tumors. Here, the $15 \mathrm{~nm}$ PEGylated NPs seem to be the most promising probes for tumor imaging in vivo. 


\section{Discussion}

\subsection{Improvement of tumor detection via pH-sensitive NIRF probes}

The present work illustrates the potential of the $\mathrm{pH}$-sensitive tumor-specific NIRF conjugate, $\mathrm{pH}-\mathrm{Her}$ (Cyp-Her5E-Herceptin) to improve tumor-detection sensitivity in vivo in comparison to the always-on probe Alexa-Her (Alexa Fluor 647-Herceptin).

\subsubsection{Tumor imaging with target specific pH-activatable NIRF probes}

The use of pH-Her enabled non-invasive detection of tumors in vivo with a 2.5 -fold increased sensitivity compared to the always-on probe Alexa-Her. The very high tumor contrast shown here by the use of $\mathrm{pH}-\mathrm{Her}$ was achieved by a considerable increase in fluorescence intensity of $\mathrm{pH}-\mathrm{Her}$ in the acidic environment of the tumor and after internalization in targeted tumor cells. At the same time $\mathrm{pH}-\mathrm{Her}$ produced a low background fluorescence in the blood, where it faces an approximately neutral $\mathrm{pH}$. The remaining autofluorescence was efficiently eliminated by subtraction of autofluorescence from prescans of mice. In contrast, the always-on fluorescence conjugate, Alexa-Her was fluorescent in the tumor as well as in the blood, as this probe did not respond to $\mathrm{pH}$ changes in the environment. Although Alexa-Her had a higher fluorescence intensity, this resulted in 2.5-fold lower CNRs in mice, compared to $\mathrm{pH}-\mathrm{Her}$, despite subtraction of autofluorescence, which was negligible compared to the high background fluorescence caused by Alexa-Her in the blood. The higher fluorescence intensity of Alexa-Her and Alexa-OH compared to $\mathrm{pH}-\mathrm{Her}$ and $\mathrm{pH}-\mathrm{OH}$ observed in this study in in vitro and in vivo measurements is due to the considerably higher quantum yield of Alexa Fluor 647 of 0.33 compared to CypHer5E which has a quantum yield of 0.27 at a $\mathrm{pH}$ of 5.3 .

Other groups, like Urano et al. ${ }^{38}$ and Ogawa and colleagues ${ }^{6}$, have also achieved high tumor to background ratios with a $\mathrm{pH}$-sensitive Herceptin-conjugate utilizing PeT for photo quenching and a TAMRA-QSY7 fluorophore-quencher pair conjugated to Herceptin, respectively. However, as these probes both emit in the visible wavelength region, the limited tissue penetration restricted the generation of in vivo data and allowed only ex vivo measurements of the opened abdominal cavity which is of low importance for noninvasive tumor detection in living organisms. Furthermore, some studies, like those of Ogawa and colleagues lack information such as CNRs or tumor to background ratios of their activatable probes, thereby giving no quantitative data about the ability of their probes to improve tumor detection?. 
The very low background fluorescence of $\mathrm{pH}-\mathrm{Her}$, which contributed to high tumordetection sensitivity was further attained by the lack of major signals in other organs in vivo and ex vivo. In contrast to that, it has been reported that some quenched fluorescence probes, like ICG coupled to Herceptin ${ }^{7}$ or Cy5.5-Herceptin-conjugates ${ }^{8}$ at high labeling densities, produce high liver signals resulting in decreased tumor contrast. Furthermore, since the applied fluorophore CypHer5E in $\mathrm{pH}-\mathrm{Her}$ emits fluorescence in the near-infrared region, the probe is well suited for in vivo imaging in deeper mouse tissues as less autofluorescence is detected and tissue penetration is increased compared to fluorophores that emit in the visible wavelength region ${ }^{21}$.

$\mathrm{pH}-\mathrm{Her}$ was well detectable in the tumor as early as one hour after probe injection in KPL-4 tumor-bearing mice. This very early detection was probably mainly due to an increase in fluorescence of $\mathrm{pH}-\mathrm{Her}$ in the acidic tumor interstitium, before receptorbased internalization in the target tumor cells occurred. It is known that CypHer5E changes its absorption spectrum and thus its excitability with changes in $\mathrm{pH}$ due to protonation/deprotonation at the nitrogen atom ${ }^{39,43}$ and therefore is able to increase its fluorescence in the presence of protons already in the acidic tumor environment. Different to $\mathrm{pH}-\mathrm{Her}$, activatable fluorescence probes based on quenching mechanisms induced by homo- or hetero-FRET ${ }^{6,8}$, $\mathrm{H}$-dimer formation ${ }^{5}$, or autoquenching ${ }^{7}$ are mainly dependent on lysosomal degradation which occurs after internalization in targeted cells. Here, processing by e.g. proteasomal cleavage leads to dequenching of the conjugates resulting in activation of fluorescence only within the cells.

In the present study the majority of Herceptin-conjugates internalized after $8 \mathrm{~h}$ of incubation at $37^{\circ} \mathrm{C}$ in HER2-positive cells in vitro, confirming previous studies which illustrate high internalization of fluorescent Herceptin-conjugates in target cells after a similar period rather than after a short time of one hour $r^{6,7}$. This indicates that the fluorescent Herceptin-conjugates activated by dequenching in the lysosomes, reported by others, need a longer time to be detected in vivo in the tumor, compared to the here used $\mathrm{pH}-\mathrm{Her}$. In the present work, the fluorescent signals in the tumor were well detectable as early as one hour after injection of $\mathrm{pH}-\mathrm{Her}$ in mice and CNRs continuously increased in the first $24 \mathrm{~h}$. In contrast to that, Ogawa and colleagues applied quenched fluorescence probes coupled to Herceptin for in vivo imaging of tumors, but did not show any fluorescence images of tumor-bearing mice, earlier than $24 \mathrm{~h}$ or $48 \mathrm{~h}$ after $i . v$. injection of the probes ${ }^{7,8}$, suggesting that tumor contrast at early times were low.

The $\mathrm{pH}$-sensitive probe used in the present study was earlier shown by others to be reversible activatable after protonation/deprotonation by changes in $\mathrm{pH}^{53}$. Although not investigated in this study, this reversible fluorescence activation might be 
especially advantageous for imaging of the localization of probes in real-time as fluorescence disappears as soon as $\mathrm{pH}-\mathrm{Her}$ escapes the acidic environment of the tumor or of endolysosomal cell compartments, which might occur in case of cell damage or cell death. In contrast, activation of fluorescence-conjugates via dequenching by lysosomal processing is irreversible and thereby does not provide imaging of environmental changes in real time.

Herceptin presents an excellent tumor targeting moiety for this study, as binding specificity and kinetics of Herceptin are well characterized ${ }^{5,38,11}$ The Herceptinconjugate, $\mathrm{pH}-\mathrm{Her}$ selectively activates its fluorescence after internalization into HER2-positive KPL-4 cells after $8 \mathrm{~h}$ at $37^{\circ} \mathrm{C}$, but not when bound to the cell membrane at $4{ }^{\circ} \mathrm{C}$, when cell physiology is slowed down and the $\mathrm{pH}$ is neutral. It has also been described by others that Herceptin-fluorophore-conjugates are internalized to a high extend after $8 \mathrm{~h}$ of incubation at $37^{\circ} \mathrm{C}$ but not at $4^{\circ} \mathrm{C}^{5}$. The spectroscopic characterizations of the $\mathrm{pH}-\mathrm{Her}$ probes, showing an increase in fluorescence only when the $\mathrm{pH}$ is decreased, are in accordance with the proposed $\mathrm{pH}$-dependent increase in fluorescence of $\mathrm{pH}-\mathrm{Her}$ after internalization in cells and point to the localization of these conjugates in acidic endolysosomal compartments. My means of Iysosomal markers $5,6,38$, this was earlier confirmed by showing that Herceptinconjuated fluorescence dyes colocalize with lysosomal compartments, which are known to have an acidic $\mathrm{pH}$ of 5 to $6^{38}$.

$\mathrm{pH}-\mathrm{Her}$ is especially advantageous for imaging purposes as a high DP ratio is not necessary for quenching of the probe. In contrast, activatable probes that utilize selfquenching via conjugation of several fluorophores to one targeting ligand need, e.g. $\sim 7$ to 20 dyes to be linked to one ligand ${ }^{8,112}$. The conjugation of many fluorophores to one ligand is challenging as, besides the higher costs for such an amount of hydrophilic dyes, some fluorophores, like ICG, form aggregates after conjugation to ligands ${ }^{113}$. The degree of aggregation is directly related to the amount of fluorophore loaded onto the protein ${ }^{113}$. In this study, a lower stability over time was observed for the $\mathrm{pH}-\mathrm{Her}$ conjugates at high DP ratios of 5.0 and 6.4, compared to $\mathrm{pH}-\mathrm{Her}$ at a low DP ratio of 1.6. The fact that $\mathrm{pH}-\mathrm{Her} \mathrm{DP} 5.0$ and 6.4 showed very low tumor targeting capacity but high bladder signals early after application in tumor-bearing mice, pointed to a structural degradation of the antibody into non-functional smaller fragments ( $<5.5 \mathrm{~nm}$ in HD) that are filtrated by the kidney rather than eliminated by the liver, representing the normal excretion route of $\mathrm{IgGs}^{75}$. The structural instability of these conjugates is likely caused by the high labeling density of Herceptin, which may lead to fragmentation of the IgG especially at the disulfide bridges (Jutta Pauli, personal communication). However, the amount of protein was also lower in the $\mathrm{pH}$ - 
Her DP 5.0 and 6.4 probes, compared to the $\mathrm{pH}-\mathrm{Her}$ conjugate at a low DP ratio and the Alexa-Her conjugates. This suggests that a part of the $\mathrm{pH}-\mathrm{Her}$ probes at high DP ratios had precipitated, probably due to enhanced agglomeration of these probes at a high labeling density (Jutta Pauli, personal communication). Most likely, both effects, structural degradation of Herceptin-conjugates into smaller non-functional fragments as well as partial precipitation of $\mathrm{pH}-\mathrm{Her}$ with high DP ratios, occurred over time. The higher stability and the increased $\mathrm{pH}$-sensitivity of $\mathrm{pH}$-Her probes at a low DP ratio, observed in this work in spectroscopic measurements favor its use for in vivo tumor detection, when compared to $\mathrm{pH}-H e r$ probes with high DP ratios.

KPL-4 cells, applied in this study to generate orthotopic breast tumor xenografts in nude mice, showed high expression of HER2 and were efficiently targeted by Herceptin-conjugated fluorophores. This confirmed the suitability of KPL-4 cells as an optimal tumor model for the evaluation of Herceptin-conjugated fluorescence labels, as already reported in previous studies ${ }^{103}$.

\subsubsection{Suppression of background fluorescence in vivo}

In the present work, tumor-detection sensitivity was improved via elimination of background fluorescence by subtraction of autofluorescence but not via LT-gated imaging. Background fluorescence was eliminated in this work, as it presents one of the greatest problems in fluorescence imaging of living mice, hampering the detection sensitivity of specific probes in vivo $^{1,2,15,16}$.

Subtraction of autofluorescence was especially advantageous in combination with the $\mathrm{pH}$-sensitive probe, $\mathrm{pH}-\mathrm{Her}$. This probe exhibited a low fluorescence outside the tumor area. Thereby, and in contrast to Alexa-Her, pH-Her had only a minor contribution to the background fluorescence and after injection of $\mathrm{pH}-\mathrm{Her}$, the majority of background signals were caused by autofluorescence of the mice. This could be measured in prescans and efficiently eliminated by a software-based subtraction, thereby leading to a high detection sensitivity of the pH-Her-derived fluorescence in the tumor. This straightforward technique is possible with the majority of imaging devices, as most imaging softwares are capable to subtract a given value of fluorescence from the generated scans. In comparison, suppression of autofluorescence by LT imaging or spectral unmixing, performed by scanning the excitation and emission spectra of signals in objects, followed by their separation via their spectroscopic signatures ${ }^{21,22}$, both require a special hardware. However, only few commercially available imaging devices are equipped with photon-counting systems, needed for LT imaging or tunable excitation or emission filters, used for 
spectral unmixing. The software based subtraction of autofluorescence represents a simple and effective alternative.

In the present study, subtraction of autofluorescence was performed by subtracting the average autofluorescence in the untreated tumor from whole scans of the same mice after probe injection. This technique may not precisely reflect the differences in autofluorescence in certain areas of the mice. As a second option, elimination of autofluorescence can be performed by subtraction of fluorescence images generated at two different excitation wavelengths, one at which mainly the autofluorescence is measured and another wavelength at which autofluorescence and probe signals can be measured ${ }^{114,115}$. However, subtraction of signals taken at different wavelengths, although they are not too far apart, is also inaccurate as the amount of autofluorescence varies at different wavelength (see section 1.1, Figure 1).

For the probes applied in this study, $\mathrm{pH}-\mathrm{Her}$ and Alexa-Her, LT-gated imaging, which offers the possibility to separate probe-derived fluorescence and autofluorescence ${ }^{2,15}$, did not improve tumor-detection sensitivity in vivo. However, in other studies, LT imaging has been successfully applied as a tool to increase detection of specific signals in vivo ${ }^{2,15}$, for example, for illustration of tumors after injection of a small peptide-fluorophore conjugate ${ }^{2}$. Moreover, compared to fluorescence intensity imaging, LT measurements provide the advantage of being relatively independent on probe concentration, thereby increasing the sensitivity for the detection of weak signals ${ }^{108}$. Nevertheless, several factors are known to influence the LT of fluorescent probes which can lead to misinterpretation of specific signals. As also shown in this study for the free dyes, $\mathrm{pH}-\mathrm{OH}$ and Alexa-OH, the LTs of fluorophores change after conjugation to Herceptin. It has also been shown that the LT of fluorescent probes can be influenced by factors, such as the presence of proteins and varying $\mathrm{pH}^{2,108,109}$. In this work, the pre-evaluation of probes under distinct conditions, such as varying $\mathrm{pH}$, the presence of proteins, and after s.c. injection in vivo, was performed in order to predict a characteristic LT and thereby to identify the fluorescence probes when distributed in vivo after i.v. injection. However, for $\mathrm{pH}-\mathrm{Her}$ in vivo, a higher LT of about 1.5 ns was measured at the tumor site compared to the predicted LT of $1.3 \mathrm{~ns}$. Therefore, in $\mathrm{pH}-\mathrm{Her}$ treated tumor-bearing mice, the majority of tumor signals was eliminated after gating of images to the predicted LT. These findings indicate, that some factors in the tumor environment influence the LT of pH-Her, which could not be assessed by the LT characterization in PBS and BSA, neither at different pH nor after s.c. injection in mice. Additional factors known to influence the LT are, amongst others, tissue depth, calcium concentration, and lipids ${ }^{16,108}$. Therefore, further studies are required to examine the conditions in detail that might have led to an increase in 
LT of $\mathrm{pH}-\mathrm{Her}$ in the tumor in vivo. As the increase in LT at the tumor site, in comparison to the predicted LT was not observed for Alexa-Her, it is likely that the sensitivity of the LT of $\mathrm{pH}-\mathrm{Her}$ to the tumor environment is a characteristic of the dye, CypHer5E. This would provide an additional potential to use CypHer5E as a highly sensitive means of identifying tumors via LT-gated imaging, due to the characteristic change in LT of the probe at the tumor site. Fluorescence probes that utilize such a principle would be highly advantageous compared to probes, like AlexaHer which resulted in high background fluorescence when circulating in the blood of mice, thereby contributing to similar LTs in the background as well as at the tumor site. LT-gated imaging using Alexa-Her did not allow an efficient separation of background signals from tumor fluorescence and thereby did not lead to an increase in tumor-detection sensitivity. Furthermore, the high background signals of the Alexa-Her conjugates are caused by the prolonged blood circulation time of $\mathrm{IgGs}^{34}$. In comparison, smaller molecules, such as peptides ${ }^{34}$ are cleared more rapidly from the blood and might be a better tumor-targeting moiety to be conjugated to always-on probes, such as Alexa Fluor. This may enable a better separation of probe-derived signals in the tumor from background signals, mainly caused by autofluorescence, according to their different $\mathrm{LTs}^{2}$ and thus improve tumor-detection sensitivity, compared to LT-gated imaging with IgG-conjugates.

In future, the $\mathrm{pH}$-sensitive NIRF probe, $\mathrm{pH}-\mathrm{Her}$ may be applied for a fast and sensitive detection of weak signals, deriving e.g. from small metastatic lesions in vivo. 


\subsection{Evaluation of Itrybe-loaded PSNPs as in vivo NIRF imaging agents}

The broadband NIR fluorophore, Itrybe, enclosed in polystyrene nanoparticles (PSNPs) of different sizes $(15,25$, and $100 \mathrm{~nm}$ ) and surface modifications was evaluated, for the first time concerning its suitability for in vivo tumor imaging. The results presented here predict a great potential for the use of Itrybe-loaded NPs for application in highly sensitive in vivo NIRF imaging. Itrybe can be excited in the NIR region at two different wavelengths and is well detectable in mice down to amounts of $1.6 \mu \mathrm{g}$ (for $15 \mathrm{~nm} \mathrm{NPs)}$.

\subsubsection{Characterization of Itrybe-loaded NPs}

Enclosure of Itrybe in PSNPs with different surface modifications $\left(\mathrm{NH}_{2}, \mathrm{PEG}\right.$, or Herceptin) had no considerable influence on the shape of spectra of Itrybe, as shown by spectroscopic analyses. This ensured a good comparability of these probes when measurements were performed with the same excitation and emission settings. The enclosure of hydrophobic Itrybe fluorophores in PSNPs enabled its application in aqueous environments and therefore in vivo. In addition to the conservation of the spectral range of the dye after enclosure in NPs, a further advantage of this NP system is its protection of Itrybe against species in the environment that might impair its fluorescence properties ${ }^{55}$.

In this study, with the exception of $15 \mathrm{~nm} \mathrm{NPs}$, none of the $\mathrm{NH}_{2}$-modifed particles showed major alteration in fluorescence with changes in $\mathrm{pH}$ or protein concentration. In the presence of BSA, 25 and $100 \mathrm{~nm}$ NPs showed a slight increase in fluorescence. The unexpected high increase in fluorescence observed for the smallest, $15 \mathrm{~nm}$ NPs in the presence of BSA is likely caused by interaction of proteins with fluorophores that are close to the surface of the small particles. As the ratio of particle surface to the volume increases with decreasing particle size (15 nm NPs have a surface area of $0.38 \mathrm{~m}^{2} / \mathrm{mg}$ PS whereas $100 \mathrm{~nm}$ NPs only have a surface of $0.06 \mathrm{~m}^{2} / \mathrm{mg}$ PS), the fluorophores in the $15 \mathrm{~nm}$ NPs have the strongest contact to its surrounding. The here performed characterization of NPs by evaluation of factors known to influence fluorescence properties is an important investigation prior to preclinical studies, as NPs used for in vivo tumor imaging are exposed to the usually acidic $\mathrm{pH}$ in the tumor and to high amounts of protein in blood and tissue.

The high amount of Itrybe molecules per NPs (which is 10 for $15 \mathrm{~nm}, 46$ for $25 \mathrm{~nm}$, and 3100 for $100 \mathrm{~nm}$ ) contributes to the high brightness of NPs. Hydrophobic dyes, like Itrybe are especially suitable for dense loading into NPs of hydrophobic PS. In 
contrast, loading with hydrophilic dyes is challenging, as the dyes commonly applied in fluorescence imaging, such as Cy- or Alexa dyes don't incorporate efficiently in the hydrophobic PS matrix. Moreover, Itrybe is favorably incorporated in NPs in high amounts, because, in contrast to most other commonly used NIR fluorophores, a close proximity of Itrybe does not result in quenching of fluorescence (Thomas Behnke, personal communication).

\subsubsection{Detection sensitivity of Itrybe enclosed in NPs}

The broad absorption spectrum of Itrybe enabled excitation of dye-loaded NPs at 635 and $670 \mathrm{~nm}$, both resulting in a well measureable fluorescence above $700 \mathrm{~nm}$. In vivo scanning revealed a stronger autofluorescence of mice at $635 \mathrm{~nm}$ compared to $670 \mathrm{~nm}$, which is in accordance with the fact that autofluorescence is reduced towards the infrared wavelength region ${ }^{1,21}$. On the contrary, fluorescence of the gastrointestinal (GI) tract of mice increased at the longer wavelength of $670 \mathrm{~nm}$ compared to the measurement at $635 \mathrm{~nm}$. Therefore, one can take advantage of the fact that Itrybe can be excited at different wavelengths. The excitation wavelength of the dye can be selected according to the imaging purpose of the probe. In the case of detection of gastric cancer for instance, an excitation wavelength of $635 \mathrm{~nm}$ may be favorable, as at this wavelength background fluorescence from food in the GI tract is minimized, that may otherwise hamper the specific detection of probe-derived signals.

Itrybe-loaded $15 \mathrm{~nm}$ NPs were well detectable in vivo down to amounts of $1.6 \mathrm{\mu g}$ when injected s.c. as well as intratumorally. For the latter, a slightly lower fluorescence intensity was measured in the tumor compared to s.c. application of the same amount of probe. This was presumably caused by absorption effects in the tumor tissue due to e.g. tumor blood vessels and the fact that the probe is distributed within the tumor in deeper layers of the mice ${ }^{9,116}$. As the $15 \mathrm{~nm}$ NPs were shown to have the lowest fluorescence intensity per mg PS, compared to the other probes, the 25 and $100 \mathrm{~nm}$ NPs would even show higher detection sensitivity in vivo.

\subsubsection{Surface functionalization of NPs}

The successful surface conjugation of ligands to Itrybe-loaded NPs is important to ensure specific binding of these probes to target structures, like tumor cells. Here, of all the probes, the $100 \mathrm{~nm}$ Herceptin-conjugated NPs showed the highest binding specificity to the HER2 antigen.

By performing an immunoassay with HER2 antigen-coated plates, the reliable evaluation of the binding specificity of Herceptin-modified NPs was possible. 
However, no absolute quantitative analysis of binding specificity of Herceptin-labeled NPs to HER2 (e.g. by determination of equilibrium constant or half maximal inhibitory concentration) was performed in this study. NPs are 3D structures allowing 3D surface conjugation of many Herceptin molecules per NP, whereas the immunoassay was performed on two-dimensional antigen-coated plates. Thus, due to sterical hindrances of the relatively large NPs bound to the antigen on the plate, the specific binding of Herceptin-coated NPs on the plate is presumably less effective than in vivo. In the animal, the 3D bioconjugation of NPs with many ligands may even result in a higher binding probability to target cells.

For the $100 \mathrm{~nm}$ NPs the number of Herceptin molecules conjugated to the surface was $~ 70$ to 100 molecules, depending on the Herceptin amount used for coupling procedure. For these probes in particular, functionalization with Herceptin was successful, with ratios between specific binding to the HER2 antigen and unspecific binding of 4.8, as measured in immunoassays. Binding specificity could not be enhanced by increasing the amount of Herceptin during the coupling procedure. Most likely, sterical hindrances did not allow further coupling of NPs with Herceptin molecules. The smaller, 25 and $15 \mathrm{~nm}$ sized probes showed low (ratio of 1.7) and no (ratio of 1.2) binding to the HER2 antigen, respectively. Since the amount of Herceptin molecules per NP was not determined for these probes (Thomas Behnke, personal communication), it is not clear whether antibody coupling to 25 and $15 \mathrm{~nm}$ NPs was successful but the antibody was non-functional after conjugation or whether Herceptin conjugation was not achieved.

This study revealed that binding of NPs of all sizes to HER2 is more efficient when Herceptin is conjugated to the NP surface using $1.5 \mathrm{kDa}$ - rather than $10 \mathrm{kDa}$ PEG chains as linker. One possible explanation may be that sterical hindrance via the large $10 \mathrm{kDa}$ PEG chains decreased binding efficiency of Herceptin to PEG. In addition, large PEG chains at a high density could form ravels with each other, further hampering the binding to Herceptin. On the other hand, conjugation of 1.5 kDa PEG chains, especially to small, $15 \mathrm{~nm}$ NPs was challenging as particles tended to agglomerate after conjugation to $1.5 \mathrm{kDa}$ PEG (Thomas Behnke, personal communication). Agglomeration might have been caused by the low electrokinetic potential (zeta potential) of the small particles, due to their low surface area, leading to lower repulsion of the particles among each other and thus to a higher agglomeration probability.

Furthermore, a reduction of PEG amounts for coupling of $15 \mathrm{~nm}$ NPs to $1 / 10$ (50 $\mathrm{nmol} / \mathrm{mg} \mathrm{PS}$ ) of the original amount did not improve Herceptin conjugation or binding specificity of particles to HER2. It may be possible that PEG was still in excess, 
leading to a too high density of PEG chains on the particle surface and thus a decreased mobility of PEG chains ${ }^{62}$. This probably resulted in steric hindrances during antibody conjugation. In future, the procedure of bioconjugation should be further optimized for small NPs, e.g. by further reducing the amount of PEG for coupling procedures.

\subsubsection{Evaluation of PSNP toxicity}

To evaluate toxicity, the impact of Itrybe-containing PSNPs on cell viability was investigated. The toxicity of the free fluorophore was not assessed, since Itrybe fluorophores were sterically incorporated in the matrix of non-biodegradable PSNPs and no leakage of the dye from the $100 \mathrm{~nm}$ NPs was observed when incubated with a $5 \%$ BSA solution for 4 weeks (Thomas Behnke, personal communication). This implies that no release of free Itrybe fluorophores occurs in vivo, which could have potentially caused any unwanted effects. $100 \mathrm{~nm}$ PSNPs bearing $\mathrm{NH}_{2}$ surface groups and PSNPs with Herceptin molecules linked to the particle surface via $1.5 \mathrm{kDa}$ PEG chains at concentrations up to $2 \mathrm{~g} / \mathrm{l}$ showed no impact on cell viability of 3T3 mouse fibroblasts after $24 \mathrm{~h}$ of incubation. These findings are in accordance with various reports in the literature, describing NPs which consist of PS as generally non-toxic and a safe tool for in vivo applications ${ }^{77,79}$. However, particle size and surface charge have to be taken into account when analyzing toxicity ${ }^{117}$, as some studies report a higher toxicity for $\mathrm{NH}_{2}$-modified PSNPs than e.g. for carboxylated PS particles, indicating that surface coating plays a prominent role in cytotoxicity ${ }^{66,78}$. However, these findings could not be confirmed in the present work, as no impact of $100 \mathrm{~nm}$ $\mathrm{NH}_{2}$ PSNPs on cell viability was observed. An increase in cytotoxicity has also been reported with a decrease in particle size ${ }^{78,79}$, as well as with increasing incubation times and concentration ${ }^{66,79}$. These findings suggest that the reduction in cell viability may be caused by an overloading of cells with NPs leading to a disturbance of cellular functions rather than by toxicity of the NPs itself ${ }^{79}$.

\subsubsection{Binding specificity of bioconjugated NPs in vitro}

Herceptin-modified $100 \mathrm{~nm}$ PSNPs specifically bound to HER2-positive, KPL-4 cells and -breast tumor tissue in vitro, whereas control probes, $\mathrm{NH}_{2}$-modified or PEGylated NPs unspecifically bound to KP-4 cells or tumors to a low degree. All probes showed low binding to HER2-negative, MDA-MB-231 cells and tumors as investigated by fluorescence microscopy. These findings are in accordance with immunoassay results, verifying specific binding of Herceptin-conjugated $100 \mathrm{~nm}$ NPs to HER2 expressed on tumor cells. Binding specificity of Herceptin-modified $100 \mathrm{~nm}$ and $25 \mathrm{~nm}$ NPs to the HER2 antigen was further proven in immunoassays by "blocking" of HER2 with 
excess of unlabeled Herceptin, thereby prohibiting specific binding of Herceptinconjugated NPs.

Moreover, unspecific uptake of PEGylated and Herceptin-modified NPs in KPL-4 and MDA-MB-231 tumor cells was observed, which increased over time. Unspecific uptake of NPs over time was also reported by Steinhauser et al. who applied NPs consisting of human serum albumin and conjugated to Herceptin. This group reported, that the unspecific uptake of PEGylated NPs increased after $3 \mathrm{~h}$. After $5 \mathrm{~h}$ of incubation, no differences between Herceptin-modified and PEGylated NPS on HER2-positive breast cancer cells could be observed. Steinhauser et al. also reported unspecific uptake of both probes in HER2-negative cells ${ }^{107}$. These findings, together with the results reported here illustrate that unspecific uptake seems to be a common phenomenon for several NP formulations.

In general, uptake of all particles by both cell types, KPL-4 and MDA-MB-231, increased over time and was reduced when cells were kept at $4{ }^{\circ} \mathrm{C}$. However, NPs showed no differences in their cellular localization under incubation at $4{ }^{\circ} \mathrm{C}$ and at 37 ${ }^{\circ} \mathrm{C}$, which indicates no predominant binding or accumulation of NPs at the cell membrane or inside the tumor cells. The fluorescence microscope used for analysis of NP-derived signals on the cellular level revealed insufficient power of depth resolution to discriminate membrane-derived signals from fluorescence deriving from inside the cell.

Enhanced fluorescence was observed on KPL-4 cells treated with $100 \mathrm{~nm}$-Herceptin NPs at early time points of incubation. Since the internalization rate of Herceptin into HER2-overexpressing cells has been reported as low as $4 \%$ per hour ${ }^{118}$, this could have predominantly been caused by antibody-receptor interaction, leading to an increase of NPs on the surface and enhanced unspecific uptake. Nevertheless, the exact way of uptake of target-specific NPs in cells is not entirely known, as reports about NP formulations of various sizes are contradictory and uptake is also known to be influenced by particle charge and material. Reports in the literature indicate that endocytosis of NPs from $43 \mathrm{~nm}$ to $150 \mathrm{~nm}$ is driven by clathrin-mediated pathways or calveolae-mediated endocytosis $66,119,120$ whereas $24 \mathrm{~nm}$ NPs consisting of PS have been reported to be internalized in a clathrin- and calveolae independent way ${ }^{120}$. Furthermore, $43 \mathrm{~nm}^{120}$ and $100 \mathrm{~nm}$ sized PSNPs were shown to colocalize with Iysosomes after internalization ${ }^{66}$, whereas small, $24 \mathrm{~nm}$ PSNPs were shown to localize outside the lysosomes but near the perinuclear space ${ }^{120}$. This indicates a dependence of NP cell uptake mechanisms on their size. Interestingly, very small NPs ( $8 \mathrm{~nm}$ ) composed of ironoxide and conjugated to PEG and Herceptin localize in lysosomes after internalization in HER2-positive tumor cells in vivo ${ }^{121}$. 
As Itrybe-loaded NPs seem to be highly and rapidly internalized in cells, they may also present suitable tools for in vivo labeling of highly endocytotic cells, like macrophages and thereby may function as sensitive markers for imaging of inflammatory diseases. Furthermore, Itrybe-loaded NPs could be applied to monitor cell tracking in vivo. Stem cells for instance could be labeled ex vivo by endocytosis and then their migration followed in vivo by imaging.

\subsubsection{Tumor targeting capacity of NPs in vivo}

None of the NPs of different sizes, $(100,25$, and $15 \mathrm{~nm})$ or surface-modifications (PEG and Herceptin) were able to visualize KPL-4 or MDA-MB-231 tumors in whole body scans after i.v. injection in mice. However, imaging revealed that all NP probes accumulate rapidly and to a high extent in the liver. This indicates that these probes still need modification in order to increase their longevity in the blood and their accumulation in the targeted tumor in vivo. For efficient delivery to the tumor, NPs should present prolonged blood circulation times, reduced nonspecific uptake in normal tissue, as well as rapid accumulation in targeted tumor tissue ${ }^{122}$.

NPs of all sizes bearing PEG surface-chains passively accumulated in MDA-MB-231 tumors after injection in mice as shown by ex vivo imaging. The highest accumulation, relative to the injected amount of probe was found in tumors of mice that received $15 \mathrm{~nm}$ NPs. This is probably due to the size of these NPs, which are small enough to allow the most efficient extravasation from the vasculature into the tumor tissue ${ }^{74}$, mediated by the enhanced permeability and retention (EPR) effect. Reports in the literature describe 20 to $100 \mathrm{~nm}$ to be the optimal size for efficient extravasation and retention of NP from capillaries to the tumor interstitium ${ }^{65}$ as well as a cutoff size of $>400 \mathrm{~nm}^{123}$. Tumor targeting with smaller NPs was also reported, e.g. for QDs of sizes of $\sim 5$ to $15 \mathrm{~nm}$ or PEGylated ironoxide NPs of $\sim 8 \mathrm{~nm}^{72,74,121}$. Furthermore, PEGylated calcium phosphate NPs of $16 \mathrm{~nm}$ accumulated in the tumor of mice with a signal persisting up to $96 \mathrm{~h}$ after probe injection ${ }^{55}$. Nevertheless, in accordance with the result of the present work, nonspecific uptake in other organs, such as liver, kidney, and spleen was also reported ${ }^{55,72,74,121}$.

Based on the ratio of tumor intensity, relative to the total intensity of the injected amount, the smallest, $15 \mathrm{~nm}$ NPs have the highest capacity for tumor targeting compared to both larger NPs. Calculations of the fluorescence intensity in the tumor relative to the injected amount of the respective probe revealed a ratio for the $15 \mathrm{~nm}$ NPs that was 4-fold higher than for the $100 \mathrm{~nm}$ particles ( 0.18 to 0.04 ) and 9-fold higher than for the $25 \mathrm{~nm}$ NPs (0.18 to 0.02). However, it should be taken into consideration that the number of NPs injected in vivo was different for each probe of different size. Thus, the injected probe of $15 \mathrm{~nm}$ NPs contained more particles than 
the 25 and $100 \mathrm{~nm}$ NP probes (in this order), causing a higher number of NPs in the blood. Therefore, the higher fluorescence observed in the tumor after injection of the $15 \mathrm{~nm}$ probe might have been influenced by the higher number of $15 \mathrm{~nm}$ NPs in the blood. However, this probe is nonetheless less fluorescent than the 25 and $100 \mathrm{~nm}$ NP probes (in this order). Therefore a direct comparison in absolute numbers of varying amounts of NPs with different sizes and fluorescence intensities is challenging and difficult. The ratio of tumor intensity, relative to the total intensity of the injected amount, was calculated to compare the tumor targeting capacity for different sizes of NPs. In addition, fluorescence reflectance imaging (FRI) of 3D structures, such as the tumor provides only semi-quantitative data, as fluorescence measurements are surface weighted ${ }^{9}$.

Even when increasing the affinity of NPs to HER2-positive KPL-4 tumors via conjugation to the tumor-specific ligand Herceptin, no clear accumulation of NPs was detected in KPL-4 tumors in vivo as well as ex vivo after $24 \mathrm{~h}$. Specific tumor targeting was also not achieved by others, who reported no clear differences between in vivo tumor-targeting capacities of target-specific NPs compared to non-targeted NPs, such as gadolinium ${ }^{124}$ or silica NPs ${ }^{125}$ coated with folic acid, or RGD labeled lipid $\mathrm{NPs}^{65}$. Specific targeting of tumors has mainly been achieved by use of QDs conjugated to tumor-specific antibodies ${ }^{72}$ or small ligands ${ }^{74}$. However, QDs, containing heavy metals, suffer from potentially toxic effects 20,75 , which limit their in vivo application.

PEGylated NPs of all sizes accumulated in MDA-MB-231 tumors when injected in mice and revealed a higher fluorescence in comparison to the autofluorescence in tumors from untreated mice. This could be shown in ex vivo measurements, where fluorescence is not absorbed by the surrounding tissue of the mouse, leading to a higher sensitivity of signal detection of excised tumors.

PSNPs used in this study are relatively inflexible spheres ${ }^{126}$, which may be one reason why they were limited in efficient extravasation from the blood and tumor penetration. In addition to the size of NPs and surface coatings, it is likely that the NP-shape and deformability is important for the probes to efficiently pass the endothelial barrier towards the tumor interstitium. Compared to the relatively rigid spheres used in this study, other groups showed that more flexible lipid NPs ${ }^{126}$ of 35 $\mathrm{nm}$ used in vivo accumulate clearly in the tumor and resulted also in high NP-derived signals in the blood plasma ${ }^{65}$. Such liposomal NP-formulations of $100 \mathrm{~nm}$ are already used for efficient delivery of anthracyclines for cancer therapy in patients ${ }^{64}$. Other examples are relatively large but deformable glycol chitosan NPs of $\sim 260 \mathrm{~nm}$, which are well detectable in vivo for up to 7 days and reveal the highest signal in the tumor compared to other organs. These flexible NPs also showed longer circulation times 
than the comparable unyielding PSNPs ${ }^{126}$ of similar size ${ }^{122}$. These observations indicate that the PSNP-sizes of up to $100 \mathrm{~nm}$, applied in the present work have the potential for tumor targeting when modified to achieve sufficient bioavailability and that the relatively low flexibility of PSNPs might have considerable influence on the tumor penetration capacity of the NPs. However, another report shows that mesoporous silica NPs of 100 to 130 can accumulate in the tumor ${ }^{125}$. Interestingly, NPs formed of anorganic silica are expected to be relatively inflexible. However, these NPs showed stronger fluorescence in the tumor than in other organs up to $24 \mathrm{~h}$ after injection $^{125}$.

These findings underline the need for detailed and systematic investigations of the in vivo behavior of nanosized carriers to gain more knowledge about the influence of composition, size, surface charge, and deformability on biodistribution and tumortargeting abilities of NPs. Therefore, all NP systems should be optimized prior to routine application in preclinical studies.

In this work, only the MDA-MB-231 tumor could be targeted by Itrybe-loaded NPs of all sizes. Histological examinations of both tumor types, MDA-MB-231 and KPL-4, revealed that vascularization was comparable, providing adequate conditions for NPs to reach both tumors through the blood system. However, in the present study, MDA-MB-231 tumors preferentially developed necrosis in the tumor center only, whereas KPL-4 tumors demonstrated necrotic areas in the tumor center as well as towards the tumor boarder. These findings confirmed previous observations for both tumor types of the frequent development of massive central necrosis ${ }^{101,127}$. One might speculate that since the effectiveness of penetration of the tumor by NPs is dependent on the tumor characteristics, the more frequent and expanded necrosis observed in KPL-4 tumors could probably hinder the efficient distribution of NPs in these tumors to a higher extend when compared to MDA-MB-231 tumors.

These results further indicate that Itrybe-loaded PSNPs are in principle capable of reaching the tumor, but that tumor targeting with Itrybe-loaded PSNPS is not very effective, presumably due to 1.) fast elimination of NPs from the organism and 2.) relatively low flexibility of PSNPs probably impairing efficient tumor penetration. Consequently, NP formulations other than of PS should be used for incorporation of Itrybe fluorophores, e.g. built of more flexible glycol chitosan or lipids. These should be evaluated for their suitability to improve the longevity of Itrybe-loaded NPs in the blood and their accumulation in the targeted tumor in vivo.

\subsubsection{In vivo biodistribution of NPs}

Biodistribution studies of Herceptin-conjugated and PEGylated NPs in healthy mice revealed a rapid uptake of all probes in the liver which was accompanied by a fast 
decrease in background fluorescence, defined as the amount of circulating probe in the blood. Knowledge about the bioavailability of Itrybe-loaded NPs, is of considerable importance for their use in tumor imaging as NPs have to be present in the bloodstream long enough to reach the targeted tumor tissue ${ }^{62}$. Uptake in nontargeted tissue should be low or entirely prevented as it causes unspecific background signals, hampering the identification of target-specific signals, deriving from the tumor.

A rapid uptake by the liver ${ }^{62,71}$ and short blood-circulation times ${ }^{71,128,129}$ have been observed in the literature for many NPs of various materials, including PSNPs ${ }^{67,130,131}$ as well. The fast liver uptake of all NPs observed in this study can be explained, because the liver is part of the excretory system and its mononuclear phagocytic system (MPS) is the basic mechanism of excretion of NPs ${ }^{122}$. NPs can be bound by opsonins in the blood serum, most commonly by IgG and complement proteins. These opsonins are then recognized by macrophages, usually Kupffer cells or macrophages of the liver that clear the particles from the blood. The rapid elimination of Itrybe-loaded NPs from the blood was most likely caused by uptake in the MPS that can occur within minutes and presents a major drawback for the accumulation of NPs at the targeted site. As shown here and by others, this rapid elimination of NPs may result in short blood-circulation times, of a few minutes ${ }^{62}$.

Biodistribution was analyzed, at early time points ( $6 \min$ to $4 \mathrm{~h}$ ) as uptake of probes occurred very rapidly after NP injection. PSNPs of all three sizes showed a rapid increase in liver signals, which remained high for $4 \mathrm{~h}$ when compared to the low background observed for all probes. Ex vivo analyses of excised organs confirmed the in vivo observations with highest signals found in the liver for all probes (average of $\sim 84 \%$ of the total intensity measured in all tested organs).

The elimination of NPs from the blood was assessed in dependence of their size, and surface modification. By comparing the distribution of NPs of different sizes, a slower uptake of NPs in the liver was found with an increase in size of NPs. The $100 \mathrm{~nm}$ NPs showed a peak intensity in the liver after $\sim 1 \mathrm{~h}$, whereas the $15 \mathrm{~nm}$ NPs peaked as early as $\sim 6$ min after probe injection in mice. This indicates that elimination of larger, $100 \mathrm{~nm}$ Itrybe-loaded PSNPs from the blood by the liver is slower compared to 25 and $15 \mathrm{~nm}$ NPs.

Liver signals measured ex vivo $4.5 \mathrm{~h}$ after probe injection were slightly lower for the small $15 \mathrm{~nm}$ NPs ( $6 \%$ and $2 \%$ lower compared to the 25 and $100 \mathrm{~nm}$ NPs, respectively), presumably due to enhanced renal filtration of the smallest NPs by the kidney. This is demonstrated by a slight increased of the kidney signal, of $\sim 3 \%$ after injection of $15 \mathrm{~nm}$ NPs compared to larger NPs. These findings are in accordance with 
the literature, showing a decrease in liver accumulation of smaller NPs ${ }^{71}$ especially at early time points after probe injection ${ }^{128}$ and also for PSNPs $^{67}$. Furthermore, accumulation of small gandolinium oxide NPs of $\sim 14 \mathrm{~nm}^{126}$ or of gold NPs of $20 \mathrm{~nm}^{71}$ was reported to occur in the kidney rather than in liver. However, high liver accumulation was described for NP-formulations with a small diameter, such as QDs of $\sim 5$ to $15 \mathrm{~nm}^{72,74,116}$. Although the results presented here, showed lower uptake in the liver of $15 \mathrm{~nm}$ NPs, compared to 25 and $100 \mathrm{~nm}$ NPs as confirmed by ex vivo analyses, the background fluorescence in vivo was also considerable low over time. This indicates that the small size of NPs did not contribute to an increased blood circulation. Work by others indicates that smaller particles tend to have longer bloodhalf-lives, presumably due to reduced plasma protein adsorption on their surface ${ }^{62,67,123}$.

Furthermore, ex vivo analyses revealed low accumulation of NPs in the spleen and a slight tendency of decreased uptake with a decrease in NPs size, as shown by the average intensities in the spleen that were $~ 8 \%, 6 \%$, and $5 \%$ for the 100,25 , and $15 \mathrm{~nm}$ NPs, respectively. Accumulation of NPs, including PSNPs ${ }^{130}$, in the spleen has been reported previously, and is likely mediated by macrophages as part of the MPS 116 , but has also been shown to be reduced with a decrease in size ${ }^{71}$. In addition to liver and spleen, uptake in the lung has been observed in this study, with an average intensity in the lung for NPs of all sizes of $\sim 3 \%$. Lung accumulation was also described for other NPs ${ }^{128}$, including PSNPs ${ }^{130}$ and may be caused by endocytosis of particles by pulmonary endothelial cells.

The results presented here reveal a change in the degree of liver uptake over time when NPs were PEGylated. For PEGylated 25 and $100 \mathrm{~nm}$ NPs, slightly higher liver signals were measured in vivo and ex vivo over time compared to the $\mathrm{NH}_{2}$ probes. Therefore, a decrease of sequestration of NPs in the liver by surface PEGylation, compared to NPs with $\mathrm{NH}_{2}$ surface groups could not be observed in the present study. These findings disaccord with the common believe that PEGylation leads to a decrease in liver signals due to avoidance of NP-elimination through the MPS, resulting in prolonged blood circulation and extravasation of NPs into permeable tissues, like the tumor ${ }^{62,123}$. PEG belongs to a group of long hydrophilic, neutrally charged polymer chains shown to be very effective in creating a surface barrier between the NP and the opsonins by "blocking" electrostatic and hydrophobic interactions on the surface and thereby preventing elimination of NP from the blood stream $^{62}$. It is known that neutrally charged particles have a lower opsonization rate than charged NPs, bearing for instance, positively charged $\mathrm{NH}_{2}$ surface groups. Furthermore, hydrophilic NPs are reported to be less recognized by opsonins, 
compared to hydrophobic ones, as blood serum proteins adsorb less on these surfaces. Apparently, as also shown in this work PEGylation does not definitively lead to a successful decrease in elimination of particles. This is in agreement with findings of Sun and colleagues who also observed no differences in biodistribution for PSNPS with sizes of 18 and $37 \mathrm{~nm}$ after PEGylation with $5 \mathrm{kDa}$ PEG (except for a reduction in spleen uptake compared to non-PEGylated probes) ${ }^{67}$.

In this work, no differences in biodistribution were observed for $25 \mathrm{~nm}$ NPs conjugated to 1.5 and 10 kDa PEG chains, both showing comparable high liver signals and low background signals over time in vivo. These results were confirmed by ex vivo analysis of organs, illustrating comparable high intensities (of 89 and 90 $\%$ of the total intensity of all organs) for the $25 \mathrm{~nm}$-PEG $1.5 \mathrm{kDa}$ and $-10 \mathrm{kDa}$ probes, respectively. Therefore, conjugation of NPs with different lengths of PEG chains had no impact on elimination of NPs by the MPS. These findings are in contrast to some reports in the literature, where PEG chains with a weight of at least $2 \mathrm{kDa}$ are required for MPS-avoidance, presumably due to a reduced flexibility of shorter PEG chains ${ }^{62,126}$.

In addition to the length of the PEG chains, their surface density may also influence recognition of probes by the MPS. As reported by Owens et al., very low surface densities of PEG result in gaps on the surface of the particle, where opsonins may bind, thereby initiating NP-elimination. On the other hand, a too high density of PEG on the surface leads to restriction of motion of the PEG chains, thereby decreasing PEG mobility and thus steric hindrance properties for opsonins. In this regard, a moderate density of PEG would be optimal, with no gaps on the surface but providing sufficient mobility of $\mathrm{PEG}^{62}$. Therefore, in future the effect of reduced amounts of surface-PEG on a prolonged bioavailability of Itrybe-loaded NPs in the blood should be investigated.

Independent on PEGylation of 15 and $25 \mathrm{~nm}$ NPs, the background fluorescence did not increase after probe injection. In contrast, application of the $100 \mathrm{~nm}$-PEG 1.5 kDa probe resulted in a slightly enhanced background fluorescence compared to the $100 \mathrm{~nm}-\mathrm{NH}_{2}$ NPs. However, this is in contrast to the finding that the PEGylated 100 $\mathrm{nm}$ NPs also showed higher liver uptake over time compared to the $\mathrm{NH}_{2}$-modified 100 $\mathrm{nm}$ NPs. Nevertheless, due to their enhanced fluorescence in the background, the PEGylated $100 \mathrm{~nm}$ NPs seem to have an increased blood circulation time, compared to all other NP probes.

Characteristic for all probes investigated here was the peak in background fluorescence within the first few minutes after probe injection, showing that the circulating NPs in the blood are rapidly eliminated. This is in accordance with reports 
of the literature for NPs built of PEGylated- $(18 \text { and } 24 \mathrm{~nm})^{67}$, serum albumin-coated$(50 \mathrm{~nm})^{131}$, or carboxylated PS $(100 \mathrm{~nm})^{130}$, as well as of PEGylated gold (20 and 80 $\mathrm{nm})^{71}$, of silica of different shape (185 and $720 \mathrm{~nm}$ in length) ${ }^{129}$, or of dextrancoated salt-bridged polymer aggregates $(100 \text { and } 500 \mathrm{~nm})^{128}$. However, longer blood circulation times were observed for some probes, such as PEGylated gadolinium NPs of $85 \mathrm{~nm}$, which in addition showed comparably low accumulation in liver, spleen, lungs and kidneys up to $8 \mathrm{~h}$ after injection ${ }^{124}$. Similar results were obtained by use of 3 and $6 \mathrm{~nm}$ sized silica NPs up to $3 \mathrm{~h}$ after injection ${ }^{132}$. Furthermore, a long half-live of NPs in the blood was reported for lipid NPs of $35 \mathrm{~nm}$ with fluorescence clearly evident in the plasma after $24 \mathrm{~h}^{65}$.

When compared to signals of single fluorophore-conjugates, like Alexa Fluor 647 coupled to Herceptin (Alexa-Her DP ratio 1.3), the background signals in this study and thus blood circulation times were relatively low for all NPs probes after i.v. injection in mice. This underlines the suggestion that the excretion of NPs was relatively fast, thereby not providing sufficient time for the NPs to effectively reach the targeted tumor.

Biodistribution analyses, performed systematically in this work, advanced the knowledge about the behavior and bioavailability of Itrybe-loaded PSNPs of different sizes and surface modifications. However, it should be mentioned, that fluorescence imaging performed in this study is not an absolutely quantitative method to assess biodistibution, mainly because different tissues (e.g. blood, plasma, and organs) absorb light in a different manner ${ }^{65}$. Of further importance for the future might be the direct analysis of fluorescence in blood samples of mice at different time points after injection of diverse NPs, as this would provide a more precise calculation of probes present in the blood pool than measurement of background fluorescence.

However, the biodistribution study explored the mechanisms for the inefficient tumor-targeting by Itrybe-loaded NPs and helped to conclude which improvements are needed for the future design of these probes to mediate tumor-detection in vivo. The overall goal is for NPs to be present in the bloodstream long enough to reach the targeted tumor tissue ${ }^{62}$. Therefore, the results obtained in this analyses, concerning the yet low bioavailability and tumor-targeting capacity of Itrybe-loaded PSNPS are helpful to further modify Itrybe-loaded PSNP probes, in regards to surface density of PEG chains or the use of more flexible materials for their design. Systematically performed studies, addressing biodistribution and suitability of novel probes for efficient tumor targeting in vivo, including NP systems, are often missing in the literature, thereby impeding clear interpretations of obtained results. 
Taken together, Itrybe-loaded NPs used in this study, demonstrate attractive probes in regard to their brightness and 3D appearance, allowing various surface modifications for in vivo imaging applications. However, the specificity and bioavailability of these probes has to be further improved to enable efficient tumortargeting in vivo. 


\section{SUMMARY AND CONCLUSIONS}

The work presented here illustrates a detailed and systematic in vitro and in vivo analysis of novel and innovative fluorescence probes, $\mathrm{pH}$-sensitive conjugates and PSNPs loaded with the broadband fluorophore Itrybe, concerning their suitability for in vivo tumor imaging. Coupled to the tumor-specific antibody Herceptin, the $\mathrm{pH}-$ sensitive dyes and Itrybe-loaded NPs were evaluated in orthotopic mouse breast tumor models for tumor-specific targeting.

This study illustrates, for the first time, the suitability of CypHer5E for its application in in vivo tumor imaging, with an enhanced sensitivity compared to commonly applied, commercially available always on fluorophores, such as AlexaFluor 647.

The $\mathrm{pH}$-sensitive Herceptin conjugate, $\mathrm{pH}-\mathrm{Her}$, only activated in the acidic $\mathrm{pH}$ of the tumor, significantly enhanced detection sensitivity of breast tumors in combination with subtraction of autofluorescence. Analysis of different methods for elimination of unwanted and perturbing autofluorescence or background fluorescence showed that especially subtraction of autofluorescence is a reliable method of image data analysis and, in combination with $\mathrm{pH}-\mathrm{Her}$, improves tumor-detection sensitivity in vivo. In contrast, as shown for $\mathrm{pH}-\mathrm{Her}$ and Alexa-Her, LT-gated imaging is more challenging, as it needs extensive pre-evaluation of LTs of applied probes under different conditions. Moreover, the results of LT-gated imaging have to be considered with caution as unknown influences, e.g. in the tumor environment may change the LT of some probes in vivo and therefore lead to misinterpretations of probe-derived signals. As a consequence, the unexpected changes in LT observed for pH-Her in tumor tissue require further investigations. Probes that display extensive drifts in LT upon distinct influences of a specific environment, such as a tumor, may have great potential for sensitive detection and monitoring of cancer in vivo when combined with LT-gated imaging. This would allow a simple separation of tumor-derived fluorescence from background signals caused by the fluorophore in the blood, according to different LTs of the probes in certain areas of the body. In future, the pH-sensitive fluorophore CypHer5E, in conjugation with other tumor-specific ligands, may be a promising tool for the sensitive detection of other malignancies as well as for monitoring of disease progression over time in vivo. The use of CypHer5E could also assist preclinical studies that evaluate novel anti-cancer therapies by detecting primary tumors as well as metastatic lesions.

This work is the first report about the in vivo application of Itrybe-loaded NPs. The results obtained here underline the potential of this probe as a highly sensitive imaging tool in vivo. As large numbers of Itrybe fluorophores can be loaded in NPs, 
very bright fluorescence probes were generated in comparison to single dye molecules. The broad spectra of Itrybe in NPs enable excitation at different wavelengths in the NIRF region and a sensitive detection of the fluorophore in vivo, thereby allowing, for example to selectively minimize unspecific signals in the GI tract. Enhanced detection sensitivity is especially important in in vivo fluorescence imaging, where autofluorescence or unspecific fluorescence from unbound probes or food decrease the detection sensitivity of probe-derived signals. Moreover, by analysis of binding specificity of Herceptin-conjugated NPs in vitro, the functionality of these probes was confirmed, excluding the lack of binding specificity as a factor that could limit or reduce tumor targeting of NPs in vivo. Although Itrybe-loaded PSNPs may present attractive tools for labeling of cells due to their high cellular uptake, their application for in vivo imaging of tumors has to be improved by increasing their bioavailability and tumor targeting capacity. In this regard, use of materials other than PS, for the generation of NPs, which exhibit e.g. more flexibility, could be advantageous. In addition, further investigations should be performed on the influences of a lower PEG surface density on biodistribution of NPs of different sizes.

In this study, specific tumor targeting to KPL4 tumors, overexpressing HER2 was achieved by coupling the probes to the tumor-specific antibody Herceptin. The application of antibody-single dye-conjugates, such as $\mathrm{pH}-\mathrm{Her}$ enabled specific targeting of tumor structures. This was possible due to a better bioavailability of these probes in vivo in comparison to Herceptin-conjugated or non-bioconjugated NPs. However, fluorophore-doped NPs also exhibit great potential for in vivo imaging and monitoring of tumors as they provide the possibility for various modifications depending on their application purpose. By combining different labels in/on one NP, such carriers could be applied for simultaneous monitoring by different imaging methods, such as fluorescence imaging and $\mathrm{MRI}^{56,57}$. They can also be surface tagged with different markers to improve binding probability to the target structures in vivo. In addition, modifications of composition of NP-formulations can advance the biodegradability of NPs in vivo and by this means their use as drug carrier systems.

In future, Itrybe-loaded NPs and CypHer5E may be used in combination with other disease-specific markers, such as antibodies or peptides for detection and evaluation of various biological processes during disease progression. 


\section{BIBLIOGRAPHY}

1 Alves, F., Napp, J. \& Mathejczyk, J. E. Optical imaging in vivo with a focus on paediatric disease: technical progress, current preclinical and clinical applications and future perspectives. Pediatric Radiology 41, 161-175 (2011).

2 Mathejczyk, J. E. et al. Spectroscopically Well-Characterized RGD Optical Probe as a Prerequisite for Lifetime-Gated Tumor Imaging. Mol Imaging 6, 469-80 (2011).

3 Zhou, L. \& El-Deiry, W. S. Multispectral fluorescence imaging. J Nucl Med 50, 1563-1566 (2009).

4 Weissleder, R., Tung, C. H., Mahmood, U. \& Bogdanov, A. In vivo imaging of tumors with protease-activated near-infrared fluorescent probes. Nature Biotechnology 17, 375-378 (1999).

5 Ogawa, M., Kosaka, N., Choyke, P. L. \& Kobayashi, H. H-type dimer formation of fluorophores: a mechanism for activatable, in vivo optical molecular imaging. ACS Chem Biol 4, 535-546 (2009).

6 Ogawa, M. et al. Fluorophore-quencher based activatable targeted optical probes for detecting in vivo cancer metastases. Mol Pharm 6, 386-395 (2009).

7 Ogawa, M., Kosaka, N., Choyke, P. L. \& Kobayashi, H. In vivo Molecular Imaging of Cancer with a Quenching Near-Infrared Fluorescent Probe Using Conjugates of Monoclonal Antibodies and Indocyanine Green. Cancer Res 69, 1268-1272 (2009).

8 Ogawa, M., Regino, C. A., Choyke, P. L. \& Kobayashi, H. In vivo targetspecific activatable near-infrared optical labeling of humanized monoclonal antibodies. Mol Cancer Ther 8, 232-239 (2009).

9 Ntziachristos, V., Ripoll, J., Wang, L. H. V. \& Weissleder, R. Looking and listening to light: the evolution of whole-body photonic imaging. Nature Biotechnology 23, 313-320 (2005).

10 Ntziachristos, V. et al. Visualization of antitumor treatment by means of fluorescence molecular tomography with an annexin V-Cy5.5 conjugate. Proc Natl Acad Sci U S A 101, 12294-12299 (2004).

11 Ntziachristos, V. Optical imaging of molecular signatures in pulmonary inflammation. Proc Am Thorac Soc 6, 416-418 (2009).

12 de la Zerda, A. et al. A comparison between time domain and spectral imaging systems for imaging quantum dots in small living animals. Mol Imaging Biol $12,500-508$ (2010).

13 Ma, G., Gallant, P. \& McIntosh, L. Sensitivity characterization of a timedomain fluorescence imager: eXplore Optix. Appl Opt 46, 1650-1657 (2007).

14 Akers, W., Lesage, F., Holten, D. \& Achilefu, S. In vivo resolution of multiexponential decays of multiple near-infrared molecular probes by fluorescence lifetime-gated whole-body time-resolved diffuse optical imaging. Mol Imaging 6, 237-246 (2007). 
15 Napp, J. et al. Time-domain in vivo near infrared fluorescence imaging for evaluation of matriptase as a potential target for the development of novel, inhibitor-based tumor therapies. Int J Cancer 8, 1958-74 (2010).

16 McCormack, E. et al. In vivo optical imaging of acute myeloid leukemia by green fluorescent protein: time-domain autofluorescence decoupling, fluorophore quantification, and localization. Mol Imaging 6, 193-204 (2007).

17 Bouchard, M. B. et al. Technical considerations in longitudinal multispectral small animal molecular imaging. J Biomed Opt 12, 051601 (2007).

18 Shaner, N. C., Steinbach, P. A. \& Tsien, R. Y. A guide to choosing fluorescent proteins. Nat Methods 2, 905-909, (2005).

19 Pauli, J. et al. Novel fluorophores as building blocks for optical probes for in vivo near infrared fluorescence (NIRF) imaging. J Fluoresc 20, 681-693 (2010).

20 Resch-Genger, U., Grabolle, M., Cavaliere-Jaricot, S., Nitschke, R. \& Nann, T. Quantum dots versus organic dyes as fluorescent labels. Nat Methods 5, 763775 (2008).

21 Weissleder, R. \& Pittet, M. J. Imaging in the era of molecular oncology. Nature 452, 580-589 (2008).

22 Phan, T. G. \& Bullen, A. Practical intravital two-photon microscopy for immunological research: faster, brighter, deeper. Immunol Cell Biol 88, 438444 (2010).

23 Morise, H., Shimomura, O., Johnson, F. H. \& Winant, J. Intermolecular energy transfer in the bioluminescent system of Aequorea. Biochemistry 13, 26562662 (1974).

24 Shu, X. et al. Mammalian expression of infrared fluorescent proteins engineered from a bacterial phytochrome. Science 324, 804-807 (2009).

25 Filonov, G. S. et al. Bright and stable near-infrared fluorescent protein for in vivo imaging. Nat Biotechnol 29, 757-761 (2011).

26 Tung, C. H. Fluorescent peptide probes for in vivo diagnostic imaging. Biopolymers 76, 391-403 (2004).

27 Kubota, K. et al. Intraoperative assessment of reconstructed vessels in livingdonor liver transplantation, using a novel fluorescence imaging technique. J Hepatobiliary Pancreat Surg 13, 100-104 (2006).

28 Dzurinko, V. L., Gurwood, A. S. \& Price, J. R. Intravenous and indocyanine green angiography. Optometry 75, 743-755 (2004).

29 Hart, L. \& Dassler, A. Mouse in Science: CANCER RESEARCH.

http://www.vetmed.ucdavis.edu/Animal_Alternatives/cancer.htm (2009).

30 Cunliffe, V. T., Furley, A. J. \& Keenan, D. Complete rescue of the nude mutant phenotype by a wild-type Foxn1 transgene. Mamm Genome 13, 245-252 (2002). 
31 TheJacksonLaboratory. Immunodeficient Model Selection: Choosing a nude, scid or Rag1 strain.

http://jaxmice.jax.org/literature/communication/communication02.pdf (2000).

32 Helson, L., Das, S. K. \& Hajdu, S. I. Human neuroblastoma in nude mice. Cancer Res 35, 2594-2599 (1975).

33 Maeda, H., Fang, J., Inutsuka, T. \& Kitamoto, Y. Vascular permeability enhancement in solid tumor: various factors, mechanisms involved and its implications. Int Immunopharmacol 3, 319-328 (2003).

34 Matsumura, Y. \& Maeda, H. A New Concept for Macromolecular Therapeutics in Cancer-Chemotherapy - Mechanism of Tumoritropic Accumulation of Proteins and the Antitumor Agent Smancs. Cancer Res 46, 6387-6392 (1986).

35 Adams, G. P. et al. High affinity restricts the localization and tumor penetration of single-chain fv antibody molecules. Cancer Res 61, 4750-4755 (2001).

36 Rudnick, S. I. \& Adams, G. P. Affinity and avidity in antibody-based tumor targeting. Cancer Biother Radiopharm 24, 155-161 (2009).

37 Kobayashi, H. \& Choyke, P. L. Target-cancer-cell-specific activatable fluorescence imaging probes: rational design and in vivo applications. Acc Chem Res 44, 83-90 (2011).

38 Urano, Y. et al. Selective molecular imaging of viable cancer cells with $\mathrm{pH}-$ activatable fluorescence probes. Nature Medicine 15, 104-109 (2009).

39 Cooper, M. E., Gregory, S., Adie, E. \& Kalinka, S. pH-sensitive cyanine dyes for biological applications. Journal of Fluorescence 12, 425-429 (2002).

40 Tung, C. H. et al. In vivo imaging of beta-galactosidase activity using far red fluorescent switch. Cancer Res 64, 1579-1583 (2004).

41 Gong, $\mathrm{H}$. et al. beta-Galactosidase activity assay using far-red-shifted fluorescent substrate DDAOG. Anal Biochem 386, 59-64 (2009).

42 Zhang, G. J. et al. In vivo optical imaging of LacZ expression using lacZ transgenic mice. Assay Drug Dev Technol 7, 391-399 (2009).

43 Nagano, T., Kiyose, K. \& Kojima, H. Functional near-infrared fluorescent probes. Chem-Asian J 3, 506-515 (2008).

44 Napp, J. et al. Targeted Luminescent NIR Polymer-Nanoprobes for In Vivo Imaging of Tumor Hypoxia. Anal Chem 23, 9039-46 (2011).

45 Lee, S. et al. A near-infrared-fluorescence-quenched gold-nanoparticle imaging probe for in vivo drug screening and protease activity determination. Angew Chem Int Ed Engl 47, 2804-2807 (2008).

46 Stock, C. et al. $\mathrm{pH}$ nanoenvironment at the surface of single melanoma cells. Cell Physiol Biochem 20, 679-686 (2007).

47 Galande, A. K., Weissleder, R. \& Tung, C. H. Fluorescence probe with a pHsensitive trigger. Bioconjug Chem 17, 255-257 (2006). 
48 Zhang, Z. \& Achilefu, S. Design, synthesis and evaluation of near-infrared fluorescent $\mathrm{pH}$ indicators in a physiologically relevant range. Chem Commun (Camb), 5887-5889 (2005).

49 Povrozin, Y. A. et al. Near-infrared, dual-ratiometric fluorescent label for measurement of pH. Anal Biochem 390, 136-140 (2009).

50 Beletskii, A. et al. High-throughput phagocytosis assay utilizing a pH-sensitive fluorescent dye. Biotechniques 39, 894-897 (2005).

51 Nordberg, E. et al. Cellular studies of binding, internalization and retention of a radiolabeled EGFR-binding affibody molecule. NuCl Med Biol 34, 609-618 (2007).

52 Gostring, L. et al. Quantification of internalization of EGFR-binding Affibody molecules: Methodological aspects. Int J Oncol 36, 757-763 (2010).

53 Hua, Y. et al. A readily retrievable pool of synaptic vesicles. Nat Neurosci 14, 833-839 (2011).

54 Lifesciences, G. H.

http://www.gelifesciences.com/aptrix/upp01077.nsf/Content/cypher_site (2011).

55 Altinoglu, E. I. et al. Near-Infrared Emitting Fluorophore-Doped Calcium Phosphate Nanoparticles for In Vivo Imaging of Human Breast Cancer. Acs Nano 2, 2075-2084 (2008).

56 Medarova, Z., Evgenov, N. V., Dai, G., Bonner-Weir, S. \& Moore, A. In vivo multimodal imaging of transplanted pancreatic islets. Nat Protoc 1, 429-435, (2006).

57 Lamanna, G. et al. Dendronized iron oxide nanoparticles for multimodal imaging. Biomaterials 32, 8562-8573 (2011).

58 Sapsford, K. E., Tyner, K. M., Dair, B. J., Deschamps, J. R. \& Medintz, I. L. Analyzing nanomaterial bioconjugates: a review of current and emerging purification and characterization techniques. Anal Chem 83, 4453-4488 (2011).

59 Santra, S. \& Malhotra, A. Fluorescent nanoparticle probes for imaging of cancer. Wiley Interdiscip Rev Nanomed Nanobiotechnol, doi:10.1002/wnan.134 (2011).

60 Pirollo, K. F. \& Chang, E. H. Does a targeting ligand influence nanoparticle tumor localization or uptake? Trends in Biotechnology 26, 552-558, (2008).

61 Hamidi, M., Azadi, A. \& Rafiei, P. Pharmacokinetic consequences of pegylation. Drug Delivery 13, 399-409 (2006).

62 Owens, D. E., 3rd \& Peppas, N. A. Opsonization, biodistribution, and pharmacokinetics of polymeric nanoparticles. Int J Pharm 307, 93-102 (2006).

63 Sharma, A., Gautam, S. P. \& Gupta, A. K. Surface modified dendrimers: synthesis and characterization for cancer targeted drug delivery. Bioorg Med Chem 19, 3341-3346 (2011). 
64 Yezhelyev, M. V. et al. Emerging use of nanoparticles in diagnosis and treatment of breast cancer. Lancet Oncol 7, 657-667 (2006).

65 Goutayer, M. et al. Tumor targeting of functionalized lipid nanoparticles: assessment by in vivo fluorescence imaging. Eur J Pharm Biopharm 75, 137147 (2010).

66 Ding, $\mathrm{H}$. et al. Bioconjugated PLGA-4-arm-PEG branched polymeric nanoparticles as novel tumor targeting carriers. Nanotechnology 22, 165101 (2011).

67 Sun, X. et al. An assessment of the effects of shell cross-linked nanoparticle size, core composition, and surface PEGylation on in vivo biodistribution. Biomacromolecules 6, 2541-2554 (2005).

68 Behnke, T. et al. Encapsulation of hydrophobic dyes in polystyrene micro- and nanoparticles via swelling procedures. J Fluoresc 21, 937-944 (2011).

69 Lee, C. M. et al. Oleyl-chitosan nanoparticles based on a dual probe for optical/MR imaging in vivo. Bioconjug Chem 22, 186-192 (2011).

70 Bringley, J. F. et al. Silica nanoparticles encapsulating near-infrared emissive cyanine dyes. J Colloid Interface Sci 320, 132-139 (2008).

71 Zhang, G. et al. Influence of anchoring ligands and particle size on the colloidal stability and in vivo biodistribution of polyethylene glycol-coated gold nanoparticles in tumor-xenografted mice. Biomaterials 30, 1928-1936 (2009).

72 Gao, X., Cui, Y., Levenson, R. M., Chung, L. W. \& Nie, S. In vivo cancer targeting and imaging with semiconductor quantum dots. Nat Biotechnol 22, 969-976 (2004).

73 Prabha, S., Zhou, W. Z., Panyam, J. \& Labhasetwar, V. Size-dependency of nanoparticle-mediated gene transfection: studies with fractionated nanoparticles. Int J Pharm 244, 105-115 (2002).

74 Choi, H. S. et al. Design considerations for tumour-targeted nanoparticles. Nature Nanotechnology 5, 42-47 (2010).

75 Choi, H. S. et al. Renal clearance of quantum dots. Nat Biotechnol 25, 11651170, (2007).

76 Liu, Y. et al. Intracellular dynamics of cationic and anionic polystyrene nanoparticles without direct interaction with mitotic spindle and chromosomes. Biomaterials 32, 8291-8303 (2011).

77 Clift, M. J. et al. The impact of different nanoparticle surface chemistry and size on uptake and toxicity in a murine macrophage cell line. Toxicol Appl Pharmacol 232, 418-427 (2008).

78 Xia, T., Kovochich, M., Liong, M., Zink, J. I. \& Nel, A. E. Cationic polystyrene nanosphere toxicity depends on cell-specific endocytic and mitochondrial injury pathways. ACS Nano 2, 85-96 (2008).

79 Sanchez-Martin, R. M., Alexander, L. \& Bradley, M. Multifunctionalized biocompatible microspheres for sensing. Ann N Y Acad Sci 1130, 207-217 (2008). 
80

81 Hanahan, D. \& Weinberg, R. A. Hallmarks of Cancer: The Next Generation. Cell 144, 646-674 (2011).

Warburg, O. On the origin of cancer cells. Science 123, 309-314, (1956).

83 Warburg, O. On respiratory impairment in cancer cells. Science 124, 269-270 (1956).

84 Gatenby, R. A. \& Gillies, R. J. Why do cancers have high aerobic glycolysis? Nat Rev Cancer 4, 891-899 (2004).

85 Schornack, P. A. \& Gillies, R. J. Contributions of cell metabolism and $\mathrm{H}+$ diffusion to the acidic pH of tumors. Neoplasia 5, 135-145 (2003).

86 Dullin, C. et al. Semiautomatic Landmark-Based Two-Dimensional-ThreeDimensional Image Fusion in Living Mice: Correlation of Near-Infrared Fluorescence Imaging of Cy5.5-Labeled Antibodies with Flat-Panel Volume Computed Tomography. Molecular Imaging 8, 2-14 (2009).

87 Stuhmer, W., Alves, F., Hartung, F., Zientkowska, M. \& Pardo, L. A. Potassium channels as tumour markers. FEBS Lett 580, 2850-2852 (2006).

88 Slamon, D. J. et al. Human breast cancer: correlation of relapse and survival with amplification of the HER-2/neu oncogene. Science 235, 177-182, (1987).

89 Slamon, D. J. et al. Studies of the HER-2/neu proto-oncogene in human breast and ovarian cancer. Science 244, 707-712 (1989).

90 Berchuck, A. et al. Overexpression of HER-2/neu is associated with poor survival in advanced epithelial ovarian cancer. Cancer Res 50, 4087-4091 (1990).

91 Le, X. F., Pruefer, F. \& Bast, R. C., Jr. HER2-targeting antibodies modulate the cyclin-dependent kinase inhibitor p27Kip1 via multiple signaling pathways. Cell Cycle 4, 87-95 (2005).

92 Harari, D. \& Yarden, Y. Molecular mechanisms underlying ErbB2/HER2 action in breast cancer. Oncogene 19, 6102-6114 (2000).

93 Bargmann, C. I., Hung, M. C. \& Weinberg, R. A. The neu oncogene encodes an epidermal growth factor receptor-related protein. Nature 319, 226-230 (1986).

94 Mendelsohn, J. \& Baselga, J. The EGF receptor family as targets for cancer therapy. Oncogene 19, 6550-6565 (2000).

95 Molina, M. A. et al. Trastuzumab (herceptin), a humanized anti-Her2 receptor monoclonal antibody, inhibits basal and activated Her2 ectodomain cleavage in breast cancer cells. Cancer Res 61, 4744-4749 (2001).

96 Segatto, O., King, C. R., Pierce, J. H., Di Fiore, P. P. \& Aaronson, S. A. Different structural alterations upregulate in vitro tyrosine kinase activity and transforming potency of the erbB-2 gene. Mol Cell Biol 8, 5570-5574 (1988). 
97 Menard, S., Casalini, P., Campiglio, M., Pupa, S. M. \& Tagliabue, E. Role of HER2/neu in tumor progression and therapy. Cell Mol Life Sci 61, 2965-2978 (2004).

98 Badache, A. \& Goncalves, A. The ErbB2 signaling network as a target for breast cancer therapy. J Mammary Gland Biol Neoplasia 11, 13-25 (2006).

99 Cho, H. S. et al. Structure of the extracellular region of HER2 alone and in complex with the Herceptin Fab. Nature 421, 756-760, (2003).

100 Hilger, I. et al. Near-infrared fluorescence imaging of HER-2 protein overexpression in tumour cells. Eur Radiol 14, 1124-1129 (2004).

101 Kurebayashi, J. et al. Isolation and characterization of a new human breast cancer cell line, KPL-4, expressing the Erb B family receptors and interleukin6. British Journal of Cancer 79, 707-717 (1999).

102 du Manoir, J. M. et al. Strategies for delaying or treating in vivo acquired resistance to trastuzumab in human breast cancer xenografts. Clin Cancer Res $12,904-916$ (2006).

103 Scheuer, W. et al. Strongly enhanced antitumor activity of trastuzumab and pertuzumab combination treatment on HER2-positive human xenograft tumor models. Cancer Res 69, 9330-9336 (2009).

104 Image] 1.42I. National Institutes of Health, USA

105 Roche, Applied Science Universal probe library. http://www.roche-applied-science.com/sis/rtpcr/upl/ezhome.htm/ (2008).

106 Invitrogen.

http://www.invitrogen.com/site/us/en/home/References/Molecular-ProbesThe-Handbook/tables/Fluorescence-quantum-yields-and-lifetimes-for-AlexaFluor-dyes.html (2011).

107 Steinhauser, I., Spankuch, B., Strebhardt, K. \& Langer, K. Trastuzumabmodified nanoparticles: optimisation of preparation and uptake in cancer cells. Biomaterials 27, 4975-4983 (2006).

108 Andersson, R. M., Carlsson, K., Liljeborg, A. \& Brismar, H. Characterization of probe binding and comparison of its influence on fluorescence lifetime of two $\mathrm{pH}$-sensitive benzo[c]xanthene dyes using intensity-modulated multiplewavelength scanning technique. Anal Biochem 283, 104-110 (2000).

109 Andersson, R. M., Carlsson, K., Liljeborg, A. \& Brismar, H. Fluorescence lifetime imaging of $\mathrm{pH}$ in cells: investigation of factors influencing the $\mathrm{pH}$ calibration lifetime. Optical Diagnostics of Living Cells Iii 1, 242-248 (2000).

110 Peters, T. All about albumin. Biochemistry, genetics and medical applications. Academic Press, 432, 9-75 (1996).

111 Yarden, Y. \& Harari, D. Molecular mechanisms underlying ErbB2/HER2 action in breast cancer. Oncogene 19, 6102-6114 (2000).

112 Hama, Y. et al. A self-quenched galactosamine-serum albumin-rhodamineX conjugate: a "smart" fluorescent molecular imaging probe synthesized with 
clinically applicable material for detecting peritoneal ovarian cancer metastases. Clin Cancer Res 13, 6335-6343 (2007).

113 Villaraza, A. J., Milenic, D. E. \& Brechbiel, M. W. Improved speciation characteristics of PEGylated indocyanine green-labeled Panitumumab: revisiting the solution and spectroscopic properties of a near-infrared emitting anti-HER1 antibody for optical imaging of cancer. Bioconjug Chem 21, 23052312 (2010).

114 Nauta, J. M. et al. In vivo photo-detection of chemically induced premalignant lesions and squamous cell carcinoma of the rat palatal mucosa. $J$ Photochem Photobiol B 39, 156-166 (1997).

115 Witjes, M. J. et al. In vivo fluorescence kinetics and localisation of aluminum phthalocyanine disulphonate in an autologous tumour model. $\mathrm{Br} J$ Cancer 73, 573-580 (1996).

116 Schipper, M. L. et al. Particle size, surface coating, and PEGylation influence the biodistribution of quantum dots in living mice. Small 5, 126-134 (2009).

117 Mayer, A. et al. The role of nanoparticle size in hemocompatibility. Toxicology 258, 139-147 (2009).

118 Mandler, R., Kobayashi, H., Hinson, E. R., Brechbiel, M. W. \& Waldmann, T. A. Herceptin-geldanamycin immunoconjugates: pharmacokinetics, biodistribution, and enhanced antitumor activity. Cancer Res 64, 1460-1467 (2004).

119 Rejman, J., Oberle, V., Zuhorn, I. S. \& Hoekstra, D. Size-dependent internalization of particles via the pathways of clathrin- and caveolaemediated endocytosis. Biochem J 377, 159-169 (2004).

120 Sahay, G., Alakhova, D. Y. \& Kabanov, A. V. Endocytosis of nanomedicines. J Control Release 145, 182-195 (2010).

121 Corsi, F. et al. HER2 expression in breast cancer cells is downregulated upon active targeting by antibody-engineered multifunctional nanoparticles in mice. ACS Nano 5, 6383-6393, doi:10.1021/nn201570n (2011).

$122 \mathrm{Na}, \mathrm{J} . \mathrm{H}$. et al. Real-time and non-invasive optical imaging of tumor-targeting glycol chitosan nanoparticles in various tumor models. Biomaterials 32, 52525261, (2011).

123 Alexis, F., Pridgen, E., Molnar, L. K. \& Farokhzad, O. C. Factors affecting the clearance and biodistribution of polymeric nanoparticles. Mol Pharm 5, 505515, (2008).

124 Oyewumi, M. O., Yokel, R. A., Jay, M., Coakley, T. \& Mumper, R. J. Comparison of cell uptake, biodistribution and tumor retention of folatecoated and PEG-coated gadolinium nanoparticles in tumor-bearing mice. $J$ Control Release 95, 613-626, (2004).

125 Lu, J., Liong, M., Li, Z., Zink, J. I. \& Tamanoi, F. Biocompatibility, biodistribution, and drug-delivery efficiency of mesoporous silica nanoparticles for cancer therapy in animals. Small 6, 1794-1805, (2010). 
126 Faure, A. C. et al. Control of the in vivo biodistribution of hybrid nanoparticles with different poly(ethylene glycol) coatings. Small 5, 2565-2575, (2009).

127 Missbach-Guentner, J. et al. Morphologic changes of mammary carcinomas in mice over time as monitored by flat-panel detector volume computed tomography. Neoplasia 10, 663-673 (2008).

128 Yaseen, M. A., Yu, J., Jung, B., Wong, M. S. \& Anvari, B. Biodistribution of encapsulated indocyanine green in healthy mice. Mol Pharm 6, 1321-1332, (2009).

129 Huang, X. et al. The shape effect of mesoporous silica nanoparticles on biodistribution, clearance, and biocompatibility in vivo. ACS Nano 5, 53905399, (2011).

130 Sarlo, K. et al. Tissue distribution of $20 \mathrm{~nm}, 100 \mathrm{~nm}$ and $1000 \mathrm{~nm}$ fluorescent polystyrene latex nanospheres following acute systemic or acute and repeat airway exposure in the rat. Toxicology 263, 117-126, (2009).

131 Ogawara, K. et al. Pre-coating with serum albumin reduces receptor-mediated hepatic disposition of polystyrene nanosphere: implications for rational design of nanoparticles. J Control Release 100, 451-455, (2004).

132 Burns, A. A. et al. Fluorescent silica nanoparticles with efficient urinary excretion for nanomedicine. Nano Lett 9, 442-448, (2009). 


\section{DANKSAgUNG}

An erster Stelle gilt mein Dank meiner Chefin Prof. Dr. Frauke Alves, die mich durch diese Arbeit begleitet und stets umsorgt, unterstützt und gefördert hat. Ich habe sehr viel von ihr gelernt.

Ich danke Prof. Dr. Walter Stühmer für die Möglichkeit meine Arbeit in seiner Abteilung durchzuführen und für das Gefühl in seiner Abteilung willkommen zu sein.

Besonders bedanken möchte ich mich bei Dr. Joanna Napp. Sie hat diese Arbeit maßgeblich betreut, stets ein besonderes Interesse an der Thematik gezeigt und sich für meine Interessen eingesetzt. Unter allen Umständen war Sie mir eine ausgezeichnete Lehrerin und Freundin.

Dr. Luis Pardo danke ich für die zahlreichen Hilfestellungen bei experimentellen und technischen Fragen.

Ein besonderer Dank gilt außerdem den Kooperationspartnern von der BAM I.5 in Berlin: Thomas Behnke danke ich für die Entwicklung der fluoreszenten Nanopartikel, das Beantworten zahlreicher Fragen über die Spektroskopie und Chemie der Proben, aber auch für die gemeinsame Weiterentwicklung des Projekts. Dr. Jutta Pauli danke ich für die Unterstützung bei der Charakterisierung der Fluoreszenzkonjugate sowie für die ergiebigen fachlichen Diskussionen. Bedanken möchte ich mich weiterhin bei Dr. Ute Resch-Genger für ihr großes Interesse an meinen Arbeiten und ihren Ideenreichtum, der maßgeblich zum Erfolg der Projekte beigetragen hat.

Mein herzlicher Dank gebührt Christian Dullin für die Ruhe, Geduld und tatkräftige Unterstützung, die er mir bei technischen, mathematischen und statistischen Fragen entgegengebracht hat, aber auch für sein kreatives Mitdenken an unterschiedlichsten Problemstellungen. Dies war mir eine große Inspiration.

Ich danke Sarah Greco, vor allem für ihre Herzlichkeit sowie für die wertvolle Unterstützung bei den Tierexperimenten und insbesondere dafür, dass ich fortwährend auf Ihre Zuverlässigkeit vertrauen konnte. Bei Bärbel Heidrich und Hanna Widera möchte ich mich für die Unterstützung bei der Durchführung von Molekular- und Zellkulturarbeiten bedanken. Im Besonderen danke ich ihnen für ihre immerwährende Bereitschaft zur Hilfestellung sowie für viel Freundlichkeit und Wärme, die sie mir entgegen gebracht haben.

Desweiteren gebührt mein Dank Dr. Roser Ufartes für ihr fortwährendes Interesse an meinen Arbeiten sowie vielen hilfreichen Ratschlägen zu deren Durchführung. Bei Dr. Fernanda Ramos-Gomez möchte ich mich für das Erstellen der Western blots und ihren Rat bei der Anfertigung der Immunfärbungen bedanken. Dr. Andrea Markus 
gebührt mein Dank für das Korrekturlesen dieser Arbeit. Gerd-Marten Kuscher danke ich für die anregenden Diskussionen und die vielen aufmunternden Worte. Bei Ursula Kutzke und Dr. Sabine Martin bedanke ich mich für die Unterstützung bei der Durchführung und Auswertung der real-time PCRs. Zur Aufarbeitung des Tumorgewebes sowie zum Anfertigen von histologischen Schnitten und Färbungen hat Rosi Streich maßgeblich beigetragen. Dafür möchte ich mich bedanken. Dr. Jeannine Mißbach-Guentner danke ich für die Unterstützung bei der Auswertung der histologischen Präparate. Des Weiteren danke ich Dr. Miso Mitkovski für seinen Rat bei mikroskopischen Fragestellungen. Mein Dank gilt zudem Ute Rust und Jörg Schischkoff für die koordinative Unterstützung während meiner gesamten Doktorarbeit. Den Pflegern der zentralen Tierstalleinrichtung in Göttingen sowie Dr. Sarah Kimmina möchte ich für die Pflege der Mäuse danken. Ein herzlicher Dank gebührt zudem Angela Senft, für die rege Mitarbeit an meinem Projekt während ihres Praktikums.

Für die großartige Zusammenarbeit bedanke ich mich bei den Mitarbeitern der Abteilungen Molekulare Biologie neuronaler Signale des Max-Planck-Instituts für Experimentelle Medizin sowie Hämatologie und Onkologie und Diagnostische Radiologie der Universitätsmedizin in Göttingen.

Prof. Dr. Junichi Kurebayashi von der Kawasaki Medical School in Japan danke ich für das freundliche Bereitstellen der KPL-4 Zelllinie. Dr. Birgit Bossenmaier von Roche Diagnostics aus Penzberg gilt mein Dank für die freundliche Bereitstellung des HER2 Antigens.

Zudem bin ich den Mitglieder meines Thesis Committees, Prof. Dr. Heidi Hahn und Prof. Dr. Martin Oppermann für das kritische und hilfreiche Begutachten meiner Forschungstätigkeiten dankbar.

An dieser Stelle möchte ich allen meinen teuren Freunden danken, für ihre Unterstützung und ihren anhaltenden Zuspruch. Hervorheben möchte ich meine "Gruppe Dino", Alexandra, Nadine, Hannes, Nils und Malte, mit denen ich die intensive Phase der Studienzeit teilen durfte. Besonderer Dank gilt hierbei Alex, die mir die letzten Jahre die beste und liebste Gefährtin war.

Nicht zuletzt gebührt mein Dank meiner Familie, insbesondere meinen Eltern, Elisabeth und Joachim sowie meiner Tante Gisela und Frank, und meinen lieben Geschwistern Lisa, Markus und Martin, sowie ihren Partnern und Kindern. Ihr habt mich unterstützt und immer an mich geglaubt, das hat mich stark gemacht. Sven, für die Kraft und den Ausgleich, die Du mir für meine Doktorarbeit und darüber hinaus gegeben hast, danke ich Dir von ganzem Herzen. 



\section{Curriculum vitae}

\section{Personal data}

Julia Eva Mathejczyk

Born: $\quad$ September $13^{\text {th }}, 1983$ in Braunschweig, Germany

\section{Profession since May 2008}

Ph.D. student at the Max-Planck-Institute for Experimental Medicine in Göttingen

Tertiary education

10/2006 - 04/2008 Georg-August-University Göttingen

Studies: $\quad$ Molecular Medicine

Degree: $\quad$ Master of Science

10/2003 - 08/2006 Georg-August-University Göttingen

Studies: $\quad$ Molecular Medicine

Degree: Bachelor of Science

\section{Secondary education}


